\title{
MedienPädagogik
}

Zeitschrift für Theorie und Praxis der Medienbildung

Lernsituationen mit Metaphern und Wikibooks.

Fallstudien zu Entwicklungspotenzialen einer integrativen Medienbildung in der Lehrerinnen- und Lehrerbildung

\section{Erster Entwurf und experimentelle Praxis}

Franco Rau

\section{Zusammenfassung}

Der vierte Teil der Arbeit präsentiert den Entwurf von Lernsituationen zur integrativen Medienbildung im Rahmen von Seminaren der erziehungswissenschaftlichen Studienanteile (Kap. 6) sowie die systematische Dokumentation der Praxiserprobung und deren wissenschaftliche Analyse (Kap. 7). Zu Beginn wird das Ziel verfolgt, die im Rahmen des Entwicklungsprojektes getroffenen didaktischen Entscheidungen nachvollziehbar darzustellen und zu begründen. Auf Basis der vorausgegangenen Kapitel werden die getroffenen Gestaltungs- und Entwicklungsentscheidungen auf den hochschuldidaktischen Handlungsebenen der «(Lehr)Veranstaltungen» sowie der "(Lern)Situationen» (Wildt 2002) formuliert. In Anlehnung an Tulodziecki et al. (2014) umfasst der Entwurf die Aspekte: Zielvorstellungen (Kap. 6.2), Annahmen zu den Voraussetzungen der Lernenden (Kap. 6.3), Annahmen zu potenziell relevanten Lern- und Lehrhandlungen (Kap. 6.4, Kap. 6.5) sowie einen Entwurf von konzeptbezogenen Seminaren (Kap. 6.6). Mit der Dokumentation der Praxiserprobung wird ein systematischer Einblick in die Realisierung des Entwurfs aus der Perspektive des Lehrenden gegeben (Kap. 7.2). Zur wissenschaftlichen Untersuchung bzw. empirischen Analyse erfolgt aufbauend auf den Ausführungen zum forschungsmethodischen Design (Kap. 3) zunächst eine Konkretisierung der spezifischen Erhebungssituationen sowie der vorgenommenen Anpassungen der Auswertungsmethoden (Kap. 7.3). Die Ergebnisse der durchgeführten Erhebungen und Analysen werden vorgestellt (Kap. 7.4) und bilden die Grundlage für die im nächsten Kapitel folgende Diskussion und Weiterentwicklung des Konzeptes (Kap. 8).

\section{Entwurf eines Seminarkonzeptes}

\subsection{Methodische Verortung}

Der Ausgangspunkt für den Entwurf war die Problematisierung der aktuellen Praxis (Abb. 6.1). Diese Problematisierung erfolgte in Betrachtung der medienpädagogischen Lehrerinnen- und Lehrerbildung an der TU Darmstadt (Kap. 4.2) sowie mit Blick auf das Spannungsverhältnis zwischen erfahrungsbasierten Vorstellungen von Studierenden einerseits und den Zielstellungen bildungswissenschaftlicher Studienanteile in der Lehrerinnen- und Lehrerbildung anderseits (Kap. 5.2). Die Formulierung und Begründung eines Entwurfs stellen den Gegenstand des aktuellen Kapitels 
dar (Abb. 6.1). Dieser basiert auf den theoretischen und empirischen Erkenntnissen der zwei vorausgegangenen Kapitel. Der Entwurf bietet zudem die Grundlage für die Durchführung des Entwicklungsprojektes. Im Prozessmodell der entwicklungsorientierten Bildungsforschung von Sesink und Reinmann $(2015,71)$ kann der folgende Entwurf als zweiter Bestandteil der ersten Phase «Einstieg in das Entwicklungsprojekt: Problematisierung und Entwurf» beschrieben werden (Abb. 6.1).

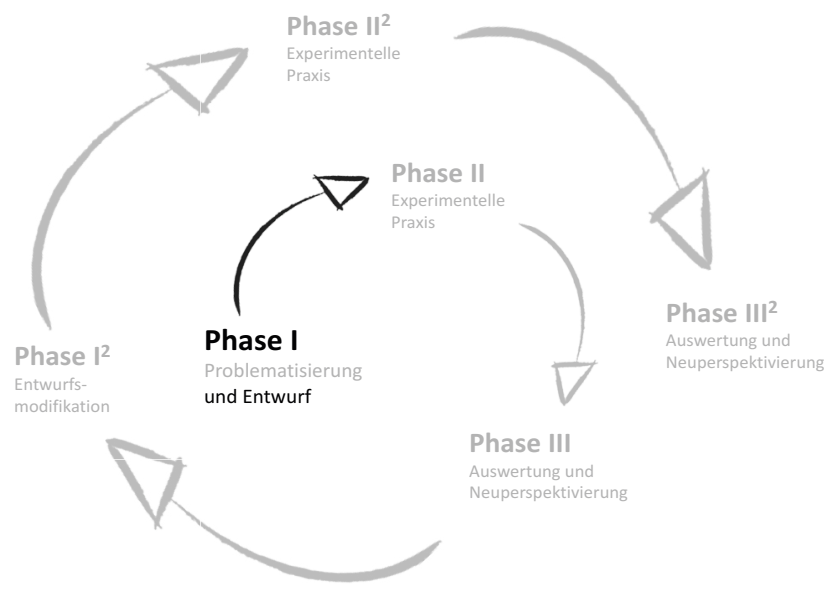

Abb. 6.1: Verortung des Kapitels im Phasenmodell der entwicklungsorientierten Bildungsforschung.

Als Orientierungsrahmen zur Darstellung des Entwurfs sowie zur angemessenen Begründung der getroffenen Entscheidungen dienen die Ausführungen von Tulodziecki et al. $(2013,2014)$ zur «Theoriegeleitete[n] Entwicklung eines Konzepts» und zum «Entwurf von konzeptbezogenen Projekten». Dafür werden Zielstellungen (Kap. 6.2), Annahmen zu den Voraussetzungen der Lernenden (Kap. 6.3) sowie theoretisch begründete Lern- und Lehrhandlungen (Kap. 6.4, 6.5) vorgestellt und diskutiert. Abschliessend wird ein konzeptbezogener Entwurf integrativer Seminare im Bereich der erziehungswissenschaftlichen Studien an der TU Darmstadt formuliert (Kap. 6.6). Dieser basiert auf den vorausgegangenen Überlegungen und berücksichtigt konkrete praktische Rahmenbedingungen hinsichtlich verfügbarer Medien (Kap. 6.6.2), inhaltlichen Entscheidungen (Kap. 6.6.3) sowie einer zeitlichen Strukturierung (Kap. 6.6.1).

Die ausführliche Darstellung der getroffenen Gestaltungs- und Entwicklungsentscheidungen zur Veränderungen von Praxis vermag aus einer wissenschaftlichen Perspektive zunächst überraschen. In Anlehnung die skizzierten Prozess- und Qualitätsstandards (Kap. 2.2) gestaltungs- und entwicklungsorientierter Bildungsforschung ist der Entwurf eines Seminarkonzeptes jedoch als relevanter Aspekt eines Forschungs- und Entwicklungsprojektes zu betrachten. Der vorgelegte Entwurf in 
diesem Kapitel 6 dient zur Erfüllung des Standards, die «Gestaltung als Bestandteil des Forschungsprozesses und der Wissensgenerierung» (Tulodziecki et al. 2013, 227) zu betrachten.

\subsection{Zielvorstellungen}

Das übergeordnete Ziel des zu entwickelnden Entwurfs ist die Ermöglichung einer integrativen Medienbildung im Rahmen von Seminaren der erziehungswissenschaftlichen Studien im Lehramtsstudium. Der Begriff integrative Medienbildung (Kap. 4.3.1) umfasst in einem bildungspolitisch bzw. administrativen Verständnis zwei Dimensionen: Durch das Lernen mit Medien soll (1.) das Lernen in den jeweiligen (Studien-) Fächern unterstützt werden und (2.) ein Lernen über Medien angeregt werden. Mit dieser übergeordneten Zielsetzung orientiert sich der Entwurf an aktuellen bildungspolitischen Leitbildern zur schulischen Medienbildung (Kap. 5.2.1). Für die theoriegeleitete Entwicklung eines Konzepts wird fachliches Lernen bzw. das Lernen im "Studienfach Erziehungswissenschaft» als (Weiter-)Entwicklung pädagogischer Artikulations- und Reflexionsfähigkeit verstanden (Kap. 6.2.1). Das Lernen über Medien kann als (Weiter-)Entwicklung der Medienkompetenz der Studierenden im Kontext sozialer Medien am Beispiel öffentlicher Wikigemeinschaften präzisiert werden (Kap. 6.2.2). Ferner sollen Studierende sinnvolle Erfahrungen zum Lernen mit Medien im Rahmen des Seminars ermöglicht werden. In der Ermöglichung dieser Erfahrungen im Rahmen der hochschuldidaktischen Praxis (Kap. 5.2.3) wird die Chance gesehen, einen Beitrag zur medienpädagogischen Professionalisierung zu leisten. In den folgenden Ausführungen wird die Relevanz dieser Zielstellung begründet und für das vorliegende Projekt konkretisiert.

\subsubsection{Pädagogische Artikulations- und Reflexionsfähigkeit}

Fachliches Lernen wird im Folgenden als (Weiter-)Entwicklung pädagogischer Artikulations- und Reflexionsfähigkeit verstanden. Dies begründet sich mit Bezug auf die Leitbilder der Lehrerinnen- und Lehrerbildung (Kap. 5.2.1). Die zentrale Aufgabe von Lehrerinnen- und Lehrern wird konsensual in der professionellen Gestaltung von Lehr- und Lernarrangements gesehen. Um diese Aufgabe professionell erfüllen zu können, sollen die erziehungswissenschaftlichen Studienanteile in der universitären Phase der Lehrerinnen- und Lehrerbildung den Erwerb von pädagogischem Wissen ermöglichen (Kap. 5.2.1). Dieses Wissen über Begriffe, Modelle und Theorien soll die Grundlage bilden, um Erfahrungen sowie Handlungsbedingungen in pädagogischen Kontexten denkend verarbeiten und beurteilen zu können (Kap. 5.2.1). Der Erwerb von Kenntnissen über historische und aktuelle Entwürfe zur Didaktik sowie zu Lernund Bildungstheorien wird explizit in den Modulhandbüchern der entsprechenden 
Lehrveranstaltungen gefordert (TU Darmstadt 2009a). Wenngleich der Erwerb von Wissen für die Entwicklung dieser Fähigkeiten keine hinreichende Bedingung darstellt, so ist sie doch eine notwendige Bedingung für professionelles pädagogisches Handeln. Eine weitere notwendige Bedingung ist die Auseinandersetzung mit den eigenen erfahrungsbasierten Vorstellungen, wie sie im Rahmen der Problematisierung (Kap. 5.2.2.2) sowie in der Diskussion theoretischer Konstrukte (Kap. 5.3.4) skizziert wurde. So können erfahrungsbasierte Vorstellungen als «Filter» wirken, welche eine Auseinandersetzung mit einer pädagogischen Perspektive erschweren. Dabei ist das Ziel nicht, erfahrungsbasierte Vorstellungen als «Fehlkonzepte» zu kritisieren, sondern Studierenden das Einnehmen einer pädagogischen Perspektive zu den eigenen Vorstellungen zu ermöglichen. So wird der Anspruch von Wildt (2003) verstanden, wenn dieser die Relationierung zwischen Subjektiven und wissenschaftlichen Theorien als Aufgabe universitärer Lehrerinnen- und Lehrerbildung formuliert (Kap. 5.2.3).

In der Diskussion Subjektiver Theorien, eigener Überzeugungen sowie metaphorischer Konzepte wurde gezeigt (Kap. 5.3), dass trotz der Differenz der Konstrukte Gemeinsamkeiten hinsichtlich praxisbezogener Folgerungen existieren. Diese bestehen darin, die erfahrungsbasierten Vorstellungen zum Ausdruck zu bringen, sodass diese einer Reflexion und Diskussion zugänglich werden (Kap. 5.3.1, 5.3.2, 5.3.3). Es wird begründet davon ausgegangen, dass die Auseinandersetzung mit Metaphern in diesem Kontext ein geeignetes Mittel ist, um die Artikulation eigener Vorstellungen zu unterstützen sowie sich den metaphorischen Gehalt unserer Alltagssprache über Erziehungs- und Bildungszusammenhänge bewusst zu machen (Kap. 5.4.3). In Anlehnung an Thomas und Beauchamp $(2011,767)$ wird die Reflexion, im Sinne der Artikulation und Exploration, der eigenen Metaphorik als wichtiges Element verstanden, um sich begründet zwischen einer «gefühlten» oder «intuitiven» Perspektive und einer professionellen pädagogischen Perspektive situativ entscheiden zu können. Die Auseinandersetzung mit der pädagogischen Fachsprache begründet sich in Anlehnung an Terhart $(1999,157)$ auch vor dem Hintergrund, dass die Wissenschaftssprache der Erziehungswissenschaft, die Berufssprache professioneller Pädagoginnen und Pädagogen sowie die Alltags- bzw. Umgangssprache über Erziehungs- und Bildungszusammenhänge kaum voneinander abgegrenzt sind (Kap. 5.5.1). Die Fähigkeit, die jeweilige Begriffsverwendung in unterschiedlichen Kontexten verstehen sowie die eigenen (pädagogischen) Vorstellungen in unterschiedlichen Kontexten artikulieren zu können, sind zentral für pädagogisch-professionelles Handeln.

Für das zu entwickelnde Seminarkonzept wurde entschieden, die (Weiter-)Entwicklung der pädagogischen Artikulations- und Reflexionsfähigkeit der Studierenden hinsichtlich folgender Teilziele zu fördern und zu unterstützen:

- Studierende werden sich ihrer eigenen Vorstellungen (als handlungsleitende Bedingungen) über Lehren, Lernen und Bildung im Kontext von Unterricht und Schule bewusst und können diese artikulieren. 
- Studierende verfügen über Kenntnisse historischer und aktueller Entwürfe zur Didaktik sowie zu Lern- und Bildungstheorien.

- Studierende sind in der Lage, Vorstellungen über Vermittlungs- und Interaktionsprozesse für pädagogisches Handeln in Unterricht und Schule unter Berücksichtigung didaktischer, lerntheoretischer und bildungstheoretischer Entwürfe zu analysieren, zu begründen und zu bewerten.

Die Unterstützung und Förderung dieser Ziele erfolgt u. a. durch den Einsatz digitaler sozialer Medien. Diese Entscheidung begründet sich zum einen hinsichtlich der didaktischen Potenziale sozialer Medien zur Anregung von Reflexionsprozessen (Kap. 4.4.2.2). Zum anderen wird mit dem Einsatz digitaler Medien das Ziel verfolgt, einen Beitrag zur medienpädagogischen Professionalisierung der Studierenden zu leisten, indem neue Erfahrungen zum innovativen Einsatz sozialer Medien in Bildungskontexten möglich werden (Kap. 4.3.2.4). In Anlehnung an das skizzierte Modell von Wildt (2003) eröffnet die Gestaltung einer innovativen Hochschulpraxis eine weitgehend vernachlässigte Chance, Studierenden neue Erfahrungsmöglichkeiten zur Gestaltung institutioneller Lehre zugänglich zu machen (Kap. 5.2.3). In diesem Zusammenhang formuliert auch Schiefner-Rohs (2012a, 44) das Plädoyer für eine angemessene hochschuldidaktische Praxis zum Lehren und Lernen mit digitalen Medien. Diese Praxis kann Studierenden neue Erfahrungen ermöglichen und als Vorbild für den Entwurf des zukünftigen Unterrichts dienen.

\subsubsection{Fähigkeit zur Teilhabe an partizipativen Medienkulturen}

Das Lernen über Medien als (Weiter-)Entwicklung der Fähigkeit zur Teilhabe an partizipativen Medienkulturen zu verstehen, begründet sich durch die vorgestellten Überlegungen zur medienpädagogischen Lehrerinnen- und Lehrerbildung. Im Rahmen der Problematisierung der Medienbildung im Lehramtsstudium wurde skizziert, dass Medienbildung ein zentrales bildungspolitisches Leitbild für die Schule darstellt. Medienbildung wird in diesem Zusammenhang als allgemeiner Erziehungs- und Bildungsauftrag beschrieben (Kap. 4.2.1.1), welcher das Lernen mit und über Medien umfasst (Kap. 4.2.1.2) und in einem integrativen Ansatz erfolgen soll (Kap. 4.2.1.3). Um diese komplexe Aufgabe pädagogisch professionell bearbeiten zu können, müssen Lehrerinnen- und Lehrern angemessen darauf vorbereitet werden. Für diese Vorbereitung ist eine medienpädagogisch und mediendidaktisch verpflichtende Lehrerinnen- und Lehrerbildung notwendig (Kap. 4.2.3). Wenn (digitale) Medien im Unterricht sowohl Gegenstand als auch Mittel sein sollen, erscheint dieser Anspruch auch für das Lehramtsstudium sinnvoll. Die Zielvorstellung ist es, zur (Weiter-)Entwicklung einer medienpädagogischen Kompetenz von Lehrkräften beizutragen. Medienpädagogische Kompetenz wird als komplexes Konstrukt mit verschiedenen Facetten 
beschrieben, bei dem die eigene Medienkompetenz als zentraler Bestandteil zu betrachten ist (Kap. 4.3.2). In Anlehnung an die Position von Mayrberger (2012a) und Moser (2010b) ist eine Auseinandersetzung mit den Partizipationspotenzialen von sozialen Medien relevant (Kap. 4.3.2, 4.5.2.1). Auch weitere Autorinnen und Autoren plädieren für eine Integration des Themas «Social Media» in die Lehrerinnen- und Lehrerbildung (Schiefner-Rohs 2013b; Mayrberger et al. 2013).

In der Diskussion aktueller Erkenntnisse zum Lernen mit sozialen Medien (Kap. 4.4) sowie zum Lernen über soziale Medien (Kap. 4.5) wurden mediendidaktische und medienerzieherische Überlegungen thematisiert. Zur praktischen Gestaltung von institutioneller Lehre ist beiden Ansätzen gemeinsam, dass eine aktive bzw. handlungsorientierte Auseinandersetzung mit sozialen Medien stattfinden sollte, um Lernen mit bzw. Lernen über Medien anzuregen. In diesem Zusammenhang wurden Wikis aus mediendidaktischer Perspektive als interessantes Instrument (Kap. 4.3.3.2) zur Anregung und Unterstützung von reflexivem und kollaborativem Lernen diskutiert (Kap. 4.4.2). Zudem kann eine handlungsorientierte Auseinandersetzung mit Wikis im Sinne von öffentlichen Sharing-Communities (Kap. 4.3.3.3) einen möglichen Zugang zu exemplarischen partizipativen Medienkulturen eröffnen (Kap. 4.5.2.1). Die Förderung der Medienkompetenz von Studierenden kann in diesem Zusammenhang als Ziel medienerzieherischer Bemühungen markiert werden. Zugleich ist die Medienkompetenz der Studierenden die Voraussetzung und das Ziel dafür, dass sie die Potenziale des Lernens mit Wikis angemessen ausschöpfen können. Lehramtsstudierende bei der Entwicklung ihrer eigenen Medienkompetenz zu unterstützen, dient in diesem Konstrukt sowohl zur Unterstützung ihres Studiums als auch zur Vorbereitung auf ihre zukünftigen Aufgaben in der schulischen Medienbildung (Kap. 4.2.1). Die Fähigkeit zur Teilhabe an partizipativen Medienkulturen wird in diesem Zusammenhang als eine Facette der medienpädagogischen Kompetenz verstanden (Kap. 4.3.2), welche vor allem als Bestandteil der eigenen Medienkompetenz der Lehramtsstudierenden zu verstehen ist.

Für das zu entwickelnde Seminarkonzept wurde entschieden, die (Weiter-)Entwicklung der Fähigkeit der Studierenden zur Teilhabe an partizipativen Medienkulturen hinsichtlich der folgenden Teilziele zu fördern und zu unterstützen:

- Studierende sind in der Lage, (öffentliche) Wikis angemessen nutzen zu können, d. h. (a) die vorhandenen Inhalte und Strukturen zu erkennen und zu verstehen und (b) die Möglichkeiten zur Interaktion und Mitgestaltung zu erkennen und zu nutzen.

- Studierende verfügen über grundlegende Kenntnisse der kollaborativen Textentwicklung und über Kenntnisse der Wissensproduktion in offenen Wikigemeinschaften (z. B. bei Wikibooks oder Wikipedia).

- Studierende sind in der Lage, kollaborativ erstellte Texte in Wikis hinsichtlich verschiedener Kriterien einschätzen und selbständig gestalten zu können. 
Die Unterstützung und Förderung dieser Ziele erfolgt ebenfalls durch den Einsatz digitaler sozialer Medien. Diese Entscheidung begründet sich hinsichtlich der skizzierten Ansätze zur handlungsorientierten Medienpädagogik (Kap. 4.5.1.2). Es wird davon ausgegangen, dass eine produktive Auseinandersetzung mit digitalen sozialen Medien hilfreich ist, um die Entwicklung der zuvor skizzierten Kompetenzen anzuregen. Zudem ist ein entsprechendes Vorgehen anschlussfähig an das von Petko (2011) formulierte Plädoyer zur Verknüpfung mediendidaktischer und medienpädagogischer Forschungsperspektiven (Kap. 2.1). Analog zur vorherigen Zielstellung (Kap. 6.2.1) begründet sich ein entsprechendes Vorgehen auch vor dem Hintergrund, einen Beitrag zur medienpädagogischen Professionalisierung der Studierenden zu leisten. So kann der innovative Einsatz sozialer Medien in der institutionellen Vermittlungspraxis der Hochschullehre den Studierenden neue Erfahrungen ermöglichen und als Vorbild zur Gestaltung der eigenen Praxis dienen (Schiefner-Rohs 2012a, 44).

\subsection{Annahmen zu den Voraussetzungen der Lernenden}

Im vorliegenden Kapitel werden die Voraussetzungen der Lernenden in Sinne von Erfahrungen, Vorstellungen und Überzeugungen von Lehramtsstudierenden (Blömeke 2004) hinsichtlich der genannten Ziele beschrieben. Dafür wird im Folgenden zwischen (1.) Kenntnissen und erfahrungsbasierten Vorstellungen zum Themenbereich der Didaktik und Bildung (Kap. 6.3.1) und (2.) Kenntnissen, Erfahrungen, Einstellungen und Nutzungsweisen von Studierenden im Umgang mit digitalen sozialen Medien (Kap. 6.3.2) unterschieden. Mit dieser Unterscheidung werden die zentralen Vorannahmen zur Konkretisierung der Lehr- und Lernhandlungen skizziert (Kap. 6.4, 6.5).

\subsubsection{Kenntnisse und Vorstellungen zu Didaktik und Bildung}

Die zu entwerfenden Lehrveranstaltungskonzepte sind in zwei Pflichtmodulen im Studienbereich der Grundwissenschaften des Lehramtsstudiengangs an der TU Darmstadt verortet (Kap. 5.2.3). In diesen Pflichtmodulen widmen sich die Studierenden im Rahmen ihres Studiums - gemäss der Empfehlung ihres Studienverlaufsplans - zum ersten Mal didaktischen sowie bildungstheoretischen Themen und Fragestellungen. Insofern kann davon ausgegangen werden, dass Studierende, die gemäss dieses Vorschlags studieren, über wenige bis keine inhaltlichen Kenntnisse und entsprechende Kompetenzen verfügen.

Im Rahmen der Problematisierung wurde in einer ersten Annäherung bereits auf das Spannungsfeld zwischen den Voraussetzungen der Lernenden einerseits sowie den Zielstellungen des erziehungswissenschaftlichen Studiums anderseits hingewiesen (Kap. 5.2). Lehramtsstudierende beginnen ihr Lehramtsstudium mit vielfältigen 
erfahrungsbasierten Vorstellungen und Erwartungen. Die Erwartungshaltung von Lehramtsstudierenden an das erziehungswissenschaftliche Studium besteht $u$. a. darin, konkretes methodisches Handlungswissen zu erwerben um als Lehrkraft handlungsfähig zu werden (z. B. Blömeke 2004; Horstkemper 2004). Diese Erwartungshaltung steht im Kontrast zu den Zielstellungen des Seminarkonzeptes (Kap. 6.2.1) sowie zum erziehungswissenschaftlichen Studium. Für Studierende mit einer entsprechenden Erwartungshaltung kann sich im Rahmen des Studiums sowie des zu entwickelnden Seminars die Frage stellen, warum die begründete Zielstellung für sie selbst sinnvoll sein sollten oder wie es Sesink und Reinmann $(2015,62)$ formulieren, inwiefern sie damit «etwas anfangen können»».

In diesem Zusammenhang wurde bereits die «Filterwirkung» erfahrungsbasierter Vorstellungen und Überzeugungen diskutiert (Kap. 5.2.2.2). Die erfahrungsbasierten Vorstellungen werden in der wissenschaftlichen Literatur häufig unter den Begriffen «Beliefs» (Kap. 5.3.1) und "Subjektive Theorien» (Kap. 5.3.2) thematisiert. Eine Gemeinsamkeit dieser Konstrukte besteht in der Annahme, dass diese die Wahrnehmung von Situationen beeinflussen und in spezifischer Weise vorbestimmen. Mit den Voraussetzungen der Lernenden sind entsprechend auch ihre Perspektiven und Überzeugungen gemeint. Diese (unbewussten) Vorstellungen können dazu führen, dass Studierende die Auseinandersetzung mit Fragen und Themen, welche nicht ihren Erwartungen entsprechen (z. B. nicht unmittelbar auf die unterrichtliche Handlungsebene gerichtet sind), für nicht relevant erachten. Zum Umgang mit dieser Herausforderung empfehlen verschiedene Autorinnen und Autoren die Thematisierung der studentischen Vorstellungen (Kap. 5.2.3).

In der Diskussion der verschiedenen Konstrukte wurde gezeigt (Kap. 5.3), dass die Artikulation der eigenen Selbstverständlichkeiten keinesfalls trivial ist. So wird mitunter angenommen, dass bestimmte Überzeugungen sowie Aspekte Subjektiver Theorien nur begrenzt bewusstseinsfähig sind. In Anlehnung an die Ausführungen von de Guerrero und Villamil $(2000,2002)$ wird davon ausgegangen, dass es über die Versprachlichung von Metaphern zumindest möglich wird, die bewusstseinsfähigen bzw. leicht zugänglichen Vorstellungen über Didaktik und Bildung zu versprachlichen (Kap. 5.4.3). Dass Studierende sowie Lehrerinnen- und Lehrern potenziell fähig sind, explizite Metaphern über Zusammenhänge von Lehren und Lernen zu formulieren, belegen zudem die theoretischen und empirischen Arbeiten zu Metaphern in der Lehrerinnen- und Lehrerbildung (Kap. 5.4). In Anlehnung an Marsch und Krüger (2007) zeigte sich aber auch, dass es nicht allen Lernenden unmittelbar gelingt, ihre Vorstellungen mithilfe von Metaphern zum Ausdruck zu bringen. 


\subsubsection{Erfahrungen und Kenntnisse zum Lernen mit und über soziale Medien}

Im Rahmen der bisherigen Ausführungen zur Medienbildung mit sozialen Medien im Lehramtsstudium (Kap. 4) wurden bereits verschiedene - durchaus konkurrierende Annahmen zu den Voraussetzungen der Studierenden hinsichtlich ihrer Erfahrungen und vorhandenen Kompetenzen zum Lernen mit und über Medien formuliert ( $z$. B. Kap. 4.2.2, 4.4, 4.5). Diese Erkenntnisse werden im Folgenden aufgegriffen, diskutiert und für den vorliegenden Entwurf konkretisiert. Der Aspekt der allgemeinen Medienkompetenz (als Bestandteil der medienpädagogischen Kompetenz) wurde in diesem Zusammenhang z. B. von Tulodziecki $(2012,282)$ perspektivisch als «Voraussetzung für ein Lehramtsstudium» betrachtet, da die Vermittlung von Medienkompetenz «letztlich in den Aufgabenbereich der Schule gehört». Für das vorliegende Projekt wird jedoch mit Bezug auf Kammerl und Ostermann (2010) sowie Herzig und Grafe (2007) nicht davon ausgegangen, dass die Schule dieser Aufgabe bereits gerecht wird und Lehramtsstudierende in angemessener Weise über medienbezogene Kompetenzen verfügen. So problematisieren Kammerl und Ostermann (2010, 49) die Voraussetzungen von Studienanfängerinnen und -anfänger im Lehramt dahingehend, dass diese nur über «wenige Kompetenzen» verfügen. Mit Bezug auf die Ergebnisse einer früheren Untersuchung (Kammerl und Pannarale 2007) weisen Kammerl und Ostermann $(2010,49)$ zudem darauf hin, dass «diejenigen Studierenden, die zu den Medienkompetenteren ihres Jahrgangs zählen, nicht Lehramt studieren». Auch Herzig und Grafe (2007) beschreiben die medienbezogenen Kompetenzen der Lehramtsstudierenden im Vergleich zu anderen Studierenden als gering:

«Die medienbezogenen Kompetenzen, über die Studierende zu Beginn des Studiums verfügen, sind insgesamt eher gering und beziehen sich vor allem auf den Umgang mit Standardprogrammen, also auf Bedienungsfertigkeiten, bei denen Studentinnen in der Regel über weniger Erfahrungen verfügen als ihre männlichen Kommilitonen. In Bezug auf Kompetenzen und im Hinblick auf Einstellungen gegenüber digitalen Medien schneiden Lehramtsstudierende bezeichnenderweise gegenüber Studierenden anderer Fächer besonders schlecht ab» (Herzig und Grafe 2007, 110).

Auch die im (bildungs-)politischen Diskurs anzutreffende Vorstellung: «Die jungen Lehrkräfte von heute kommen aus einer Generation, die mit Medien aufgewachsen ist, und die deshalb eine bessere Medienkompetenz für den Lehrerberuf mitbringen», welche von Niesyto $(2012,14)$ berichtet wird, muss in Betrachtung empirischer Studien als Wunschvorstellung bezeichnet werden. Entsprechende Generationsvorstellungen werden auch unter den Begriffen «Net Generation» (Tapscott 1998) oder "Digital Natives» (Prensky 2001) diskutiert. Neben der Annahme, dass Studierende durch das Aufwachsen in einer digital geprägten Kultur über Medienkompetenz verfügen, wird ihnen auch ein neues Lernverhalten sowie eine Affinität zum Lernen mit 
digitalen Medien unterstellt. Entsprechende Generationskonzepte verbleiben jedoch weitgehend auf der Ebene von Behauptungen und stellen keine empirisch fundierten Erkenntnisse dar. Beispielsweise markiert Schulmeister (2009a) verschiedene forschungsmethodische Schwächen diesbezüglicher Arbeiten. Die vorgenommenen generalisierenden Schlussfolgerungen von Autoren wie Tapscott (1998) oder Prensky (2001) sind insofern nicht haltbar. Ferner problematisiert Jenkins (2007), dass die Metapher der «digitalen Eingeborenen» oder der «digitalen Generation» sowohl den "digital divide» als auch den «participation gap (in young people's access to the social skills and cultural competencies needed to fully and meaningfully participate in the emerging digital culture)» (Absatz 6) ignoriert. Entsprechende Vorstellungen verdecken ebenfalls notwendige Differenzierungen zwischen neuen Formen einer «participatory culture of fans, bloggers, and gamers» (Jenkins 2007, Absatz 7) sowie die Berücksichtigung der Diversität von Studierenden bei der Gestaltung und Planung von Lehrveranstaltungen (Schulmeister 2009a). Weitere Autorinnen und Autoren kritisieren und problematisieren ebenfalls die Vorstellung von Generationskonzepten (z. B. Arnold 2011; Reinmann 2010; Schulmeister 2009b; Ebner et al. 2008).

Bei Betrachtung verschiedener Untersuchungen an deutsch- und englischsprachigen Universitäten finden sich jedoch Indizien dafür, dass zukünftige Studierende auf der technischen Ebene zunehmend besser ausgestattet sind (Budka et al. 2011). Beispielsweise heben Ebner et al. (2008) in ihrer Studie an der TU Graz hervor: «Die oftmals gepriesene (Net Generation` lässt auf sich warten bzw. spiegelt eher eine technisch besser ausgestattete wider» (Ebner et al. 2008, 123). Ein ähnliches Ergebnis bietet die Untersuchung von Grosch und Gidion (2011) am Karlsruher Institut für Technologie. Ein zentraler Befund ihrer Studie lautet: «KIT-Studierende verfügen im Sinne einer Vollversorgung über die nötige technische Infrastruktur zur Nutzung der für das Studium relevanten Mediendienste» (Grosch und Gidion 2011, 51) ${ }^{157}$. Dieses Ergebnis umfasst sowohl die Nutzung der digitalen Angebote auf dem Campus sowie bei den Studierenden zuhause. Auf Basis einer Befragung von 2339 Studierenden an verschiedenen deutschen Universitäten und Hochschulen kommen auch ZawackiRichter et al. (2014) zu dem Ergebnis, dass «die Studierenden sehr gut mit digitalen Endgeräten ausgestattet [sind]. Ein Drittel der Studierenden besitzt sogar mehr als sechs verschiedene Geräte» (Zawacki-Richter et al. 2014, 10) ${ }^{158}$. Für die Planung aktueller Lehrveranstaltung wird auf Basis der skizzierten Studienergebnisse davon ausgegangen, dass die Studierenden über eine sehr gute technische Ausstattung

157 Dies zeigt sich in Betrachtung der erhobenen Daten u. a. daran, dass etwa $85 \%$ der befragten Studierenden über ein Notebook/Laptop verfügen. Von den 1372 befragten Studierenden gaben nur 2,6\% an, dass sie weder über Notebook/Laptop noch über Desktop-PC/Computer zuhause verfügen (Grosch und Gidion 2011, 54). Im Vergleich mit weiteren Daten zeigt sich auch, dass die Karlsruher Studierenden «über eine ähnliche Ausstattung mit Computergeräten verfügen wie die vergleichbare Alterskohorte oder die Studierenden insgesamt» (Grosch und Gidion 2011, 52).

158 Im Rahmen der Untersuchung von Zawacki-Richter et al. (2014) wurde u. a. nach dem Besitz von Notebook/Laptop, Desktop-PC, Smartphone sowie Druckern und Scannern gefragt. 
verfügen. Diese Ausstattung umfasst einen Zugang zum Internet sowie dessen regelmässige Nutzung. Diese Annahme belegen die empirischen Daten der bereits zitierten Erhebungen von Grosch und Gidion (2011) und Zawacki-Richter et al. (2014). Auch in empirischen Erhebungen hinsichtlich ähnlicher Alterskohorten finden sich seit Jahren Belege dafür, dass der Zugang zum Internet sowie die Nutzung des Internets für Jugendliche und junge Erwachsene alltäglich geworden ist (z. B. Peterhans und Sagl 2011; van Eimeren und Frees 2010).

Trotz der sehr guten technischen Ausstattung nutzen die Studierenden nur ein begrenztes Spektrum der vielfältigen Möglichkeiten im Umgang mit dem Internet bzw. im Umgang mit sozialen Medien. Die Möglichkeiten zur produktiven und gestalterischen Nutzung von sozialen Medien werden kaum wahrgenommen (Kap. 4.5.2.1). Dies zeigt sich bei Betrachtung des Nutzungs- und Lernverhaltens von Studierenden. Schulmeister (2009b) zeigt auf, dass trotz steigender Nutzungszahlen in Social Communities wohl kein «Heer an Internet-Enthusiasten» (Schulmeister 2009b, 140) auf die Hochschulen zukommen wird. Die Studierenden besitzen eine pragmatische Haltung gegenüber dem Gebrauch neuer Medien. Es sind vor allem die als nützlich empfundenen Anwendungen zur Kommunikation und Informationssuche, welche häufig und gern verwendet werden (Schulmeister 2009b). Diese Einschätzung wird durch die Daten der HISBUS-Studie zum «Studieren im Web 2.0» gestützt (Kleimann et al. 2008). Exemplarisch hervorheben lässt sich dabei, dass die Online-Enzyklopädie Wikipedia (60\%) sowie die Social Communities ${ }^{159}(51 \%)$ von Studierenden am häufigsten verwendet werden (Kleimann et al. 2008, 5). Dabei erfolgt die Nutzung von sozialen Netzwerken insbesondere zur Kontaktpflege, z. B. zur «Kommunikation mit Freunden (72\%)» (Kleimann et al. 2008, 6). Bei der Nutzung von Wikipedia steht für Studierende das «Lesen von Artikeln eindeutig im Vordergrund ( $80 \%$ )» (Kleimann et al. 2008, 7). Eine Erstellung oder Bearbeitung bestehender Artikel sowie eine Beteiligung an Diskussionsseiten erfolgt selten durch Studierende. Stattdessen gaben 77 $\%$ der Studierenden an, noch nie einen Beitrag editiert zu haben. $83 \%$ der befragten Studierenden haben noch nie einen Diskussionsbeitrag geschrieben und $83 \%$ haben noch nie einen neuen Artikel beigesteuert (Kleimann et al. 2008, 7). Die Akzeptanzanalyse von Grosch und Gidion (2011) liefert ähnliche Ergebnisse zur produktiven und gestalterischen Möglichkeiten zur Nutzung sozialer Medien:

«Bezüglich der Akzeptanzwerte hat es den Anschein, dass Web 2.0-Angebote die rein rezeptiv nutzbar sind und primär der Informationsversorgung dienen i.d.R. hohe Werte aufweisen während Web 2.0-Angebote, die stärker auf Kommunikation, Interaktion und Kooperation ausgerichtet sind, mehr zu mittleren bis schwachen Werten tendieren. Dies spricht außerdem, zumindest bezogen auf die Nutzung im Studium, gegen das gängige Verständnis des Web 2.0 als 〈Mitmach-Web〉 》 (Grosch und Gidion 2011, 74).

159 Im Sinne des skizzierten Begriffsverständnisses von Webanwendungen und Apps zur Knüpfung und Pflege von Kontakten (Kap. 4.3.3.1). 
Auf Basis der skizzierten Daten wird davon ausgegangen, dass Studierende wenig Erfahrungen mit der produktiven Nutzung von Sharing-Communities in Form von Wikis und vergleichbaren Angeboten haben. Zum anderen wird angenommen, dass Studierende nur über wenig Erfahrungen und Kenntnisse zum Lernen mit digitalen sozialen Medien im Kontext konkreter Seminare und Lehrveranstaltungen verfügen. Wenngleich Studierende Erfahrungen mit sozialen Netzwerken haben und diese in vielfältiger Weise zum Austausch nutzen, werden kaum Handlungen im Sinne eines aktiven, partizipativen oder reflexiven Lernens (Kap. 4.4.2.1, 4.4.2.2) in empirischen Befragungen sichtbar. In der Untersuchung von Zawacki-Richter et al. $(2014,19)$ wird in diesem Zusammenhang die Aussage formuliert, dass $82 \%$ der Studierenden angegeben haben, «dass sie sich auch über Angelegenheiten im Studium austauschen». Zu den studienbezogenen Aktivitäten mit sozialen Netzwerken gehörten gemäss der Daten des HISBUS-Studie u. a. die «Klärung von Fragen für das Selbststudium (59 \%), die Prüfungsvorbereitung $(55 \%)$ und der Austausch von Dokumenten und Literatur (49\%)» (Kleimann et al. 2008, 6). Statt für inhaltliche Diskussionen und gemeinsames Lernen scheinen Studierende über soziale Netzwerke insbesondere organisatorische Fragen zu verhandeln. Ein Grund für die ausbleibende Nutzung der Lern- und Partizipationsmöglichkeiten liegt nach Jones et al. (2010) in der Trennung zwischen «social life and higher educational process». Diese Aussage war eines der Ergebnisse ihrer Interviewstudie mit Studierenden an englischen Universitäten. Berlanga et al. (2010) weisen in diesem Zusammenhang darauf hin, dass der Einsatz von Social Software bzw. sozialen Medien insbesondere eine Herausforderung für Lernende darstellt: «Importantly, rather than as a technological, instructional design, or institutional challenge, the inclusion of Web 2.0 tools into university practice is perceived as a challenge for learners» (Berlanga et al. 2010, 199).

Eine weitere Annahme über die Voraussetzungen der potenziellen Lehramtsstudierenden betrifft die (fehlenden) Schulerfahrungen zum Lernen mit und über digitale (soziale) Medien. Es wird davon ausgegangen, und diese Annahme kann eine mögliche Begründung für die Schwierigkeiten vieler Studierenden markieren, dass nur sehr wenige Lernende in institutionellen Vermittlungssituationen ihrer Schule regelmässig Kontakt mit digitalen (sozialen) Medien hatten. Diese Annahme begründet sich durch verschiedene empirische Untersuchungen. Beispielsweise befragte Blömeke (2005) im Wintersemester 1998/1999, Lehramtsstudierende an der Universität Paderborn zu ihren «themenbezogene[n] Erlebnisse[n] und deren Bewertung». Den Umfang des Computereinsatzes im Unterricht beurteilten 82,7\% der 173 befragten Lehramtsstudierenden mit «nie» bzw. «selten» (Blömeke 2005, 7). Lediglich eine Person schätze den Umfang des Computereinsatzes als «oft» ein. Wenngleich diese Zahlen zum Zeitpunkt der Planung für das vorliegende Projekt veraltet erscheinen, ist der Einsatz digitaler Medien im Unterricht seitdem längst nicht alltäglich geworden. Ein zentrales Ergebnis der ICILS-Studie im Jahr 2013 war u. a., dass die Häufigkeit 
des Computereinsatzes in Schulen im internationalen Vergleich unterdurchschnittlich ist: «Nur ein Drittel (34.4\%) der Lehrpersonen nutzt regelmäßig (mindestens wöchentlich) Computer im Unterricht, nur 9.1 Prozent täglich» (Eickelmann et al. 2014, 20). Auch die Förderung von computer- und informationsbezogener Kompetenzen ist problematisch: «Lediglich etwa ein Drittel der Lehrpersonen in Deutschland gibt an, dass sie nachdrücklich verschiedene IT-bezogene Fähigkeiten [...] fördern» (Eickelmann et al. 2014, 21). Gemäss der Daten des Länderindikators (Telekom Stiftung 2015) nutzen aktuell 47,6 \% der Lehrerinnen- und Lehrern im bundesweiten Durchschnitt mindestens einmal pro Woche einen Computer im Unterricht (Telekom Stiftung 2015, 12). In Hessen sind es allerdings nur $24 \%$ der Lehrkräfte, die mindestens einmal pro Woche einen Computer im Unterricht nutzen. Zudem gehört Hessen jeweils zu den Ländergruppen, bei denen die wenigsten Lehrerinnen- und Lehrern angegeben haben, genügend Zeit zur Vorbereitung computerunterstützter Unterrichtsstunden zu haben (ebd., 14) und sich die meisten Lehrkräfte «mehr Unterstützung für den Einsatz von Computern im Unterricht wünschen» (ebd., 16).

Auch weitere empirische Untersuchungen bieten Hinweise darauf, dass nur wenige Schülerinnen und Schüler in ihrer Schulzeit regelmässig mit digitalen Medien lernen und arbeiten. Im Rahmen der ICILS-Studie beschreiben lediglich $31.4 \%$ der Achtklässlerinnen und Achtklässler die Computernutzung in der Schule als regelmässig und nur $1.6 \%$ gaben an, Computer täglich zu nutze (Eickelmann et al. 2014, 20). Die JIM-Studie (MPFS 2014) zeigt in diesem Zusammenhang auch die deutliche Diskrepanz zum Medienhandeln innerhalb und ausserhalb der Schule auf. Während insgesamt «81 Prozent der Zwölf- bis 19-Jährigen das Internet täglich, weitere 13 Prozent mehrmals pro Woche» (MPFS 2014, 23) nutzen, sind es in der Schule deutlich weniger. Der Austausch mit anderen über den Unterricht wurde von $17 \%$ der befragten Jugendlichen angegeben und markiert damit die Tätigkeit, die von den meisten Jugendlichen täglich oder mehrmals die Woche erfolgt (MPFS 2014, 32). Nahezu ein Drittel der Schülerinnen und Schüler berichten, zumindest alle 14 Tage Texte am Computer bzw. im Internet zu schreiben (MPFS 2014, 32). Interessant ist dabei, dass das Schreiben von Texten im Gymnasium seltener als in anderen Schulformen erfolgt: «So berichten nur zwölf Prozent der Gymnasiasten, dass sie regelmäßig am Computer Texte schreiben, bei den anderen Schulformen sind es mit 18 Prozent etwas mehr» (MPFS 2014, 33). Unabhängig von den Schulformen zeigt sich auf Basis der JIM-Studie zudem, dass die regelmässige Nutzung des Internets im Unterricht (12-13 Jahre: 7 \%, 18-19 Jahre: $28 \%$ ) sowie das digitale Schreiben von Texten (12-13 Jahre: 11 \%, 18-19 Jahre: $20 \%$ ) mit dem Alter der Schülerinnen und Schüler zunimmt (MPFS 2014, 32 f.). Entsprechend dieser Daten wird davon ausgegangen, dass nur wenige Studierende über Erfahrungen mit dem Schreiben von Texten über soziale Medien haben. 
Weitere empirische Untersuchungen thematisieren die Einstellungen von Lehrkräften und Lehramtsstudierenden gegenüber digitalen Medien sowie Medienbildung als Vermittlungsaufgabe. Bezogen auf die Einstellung kritisieren Kammerl und Ostermann $(2010,49)$ die aktuelle «Schulpraxis, in der Medienbildung nur gering in den Fachunterricht integriert ist und eher als additive Aufgabe betrachtet wird». Zudem wird die Umsetzung einer Medienbildung in Schulen, so die Kritik von Kammerl und Ostermann $(2010,49)$, «derzeit noch kaum oder gar nicht überprüft». Im Gegensatz zu den skizzierten Leitbildern (Kap. 5.2.1) wird Medienbildung in der Praxis nicht als genuiner Bestandteil eines jeden Fachunterrichts verstanden. In Betrachtung der Einstellung von Lehramtsstudierenden hinsichtlich (digitaler) Medien verweist Niesyto $(2012,345)$ «auf den Beharrungsfaktor medialer Habitusformen bei vielen Lehramtsstudierenden, die [...] einen aktiven und differenzierten Umgang mit neuen Medien erschweren». Ferner wurde mit Herzig und Grafe (2007) bereits auf die problematischen Unterschiede bezüglich der Einstellung von Lehramtsstudierenden hinsichtlich digitaler Medien im Vergleich zu anderen Studienfächern hingewiesen. Ein relevanter Einflussfaktor auf die zurückhaltenden Einstellungen von Lehramtsstudierenden sehen Herzig und Grafe (2007) in den fehlenden Erfahrungen. Diesen Zusammenhang beschreiben Herzig und Grafe (2007) wie folgt:

«Betrachtet man [...] Studierende in ihren Einstellungen zu digitalen Medien, so schätzen diese die Vermittlungsfunktion digitaler Medien - insbesondere von Lernprogrammen - als durchaus nützlich ein, zeigen sich aber deutlich skeptischer im Hinblick auf die Förderung von Motivation und Selbstständigkeit. Allerdings ist - in Übereinstimmung mit den anderen Personengruppen - die Einstellung deutlich abhängig von der eigenen Erfahrung. Vor diesem Hintergrund kann die teilweise etwas zurückhaltende Einschätzung der Studierenden auch Ausdruck einer an den Hochschulen noch nicht durchgängig überzeugenden Nutzung digitaler Medien in der Lehre sein» (Herzig und Grafe 2007, 34).

Zusammenfassend wird davon ausgegangen, dass Lehramtsstudierende nur über eine geringe Medienkompetenz verfügen und nur wenige Studierende bereits mit den produktiven und partizipativen Potenzialen sozialer Medien vertraut sind. Zudem wird davon ausgegangen, dass nur wenige Studierende über Erfahrungen zum Lernen mit sozialen Medien im Studium sowie über Erfahrungen zum Lernen mit dem Internet im Allgemeinen verfügen. Die fehlenden Erfahrungen können auch ein Grund dafür sein, dass die Mehrheit der Lehramtsstudierenden eine eher zurückhaltende Einstellung gegenüber digitalen Medien hat. Umso wichtiger ist es, dass das Lernen mit digitalen Medien ein zentrales Gestaltungsziel darstellt, um Studierenden neue Erfahrungen zu ermöglichen (Kap. 1.2, 6.2). 


\subsection{Lernhandlungen}

Der Begriff Lernhandlungen bzw. Lernaktivitäten umfasst in Anlehnung an Herzig $(1998,175)$ und Grafe $(2008,141)$ alle Aktivitäten, die auf einen spezifischen Lernzusammenhang bzw. Lernprozess gerichtet sind. Im Folgenden wird hinsichtlich der formulierten Zielstellungen (Kap. 6.2) zwischen zwei Lernzusammenhängen unterschieden:

- Lernen als (Weiter-)Entwicklung pädagogischer Artikulations- und Reflexionsfähigkeit.

- Lernen als (Weiter-)Entwicklung der Fähigkeit zur Teilhabe an einer partizipativen Medienkultur.

Für beide Zielstellungen wird davon ausgegangen, dass die Entwicklung entsprechender Fähigkeiten mit sozialen Medien unterstützt werden kann. In Anpassung der Projekte von Bonk et al. (2009) und Xiao und Lucking (2008) wird dafür ein projektorientiertes Vorgehen gewählt. Das Ziel des Projektes ist es, gemeinsam mit Studierenden ein Buchprojekt zum Themenbereich Lehren, Lernen und Bildung im Rahmen eines öffentlichen Wikis ${ }^{160}$ zu entwickeln. Ausgehend von den zwei benannten Zieldimensionen werden im Folgenden Handlungen von Studierenden konkretisiert, welche potenziell Lernprozesse anregen können. Die Überlegungen basieren auf den Erkenntnissen der vorherigen Kapitel zu den Reflexions- und Artikulationsmöglichkeiten von Metaphern in den pädagogischen Studienanteilen der Lehrerinnen- und Lehrerbildung (Kap. 5) sowie zum Lernen mit und über soziale Medien in der Hochschule (Kap. 4).

\subsubsection{Pädagogische Artikulations- und Reflexionsfähigkeit}

Der Begriff der pädagogischen Artikulations- und Reflexionsfähigkeit wurde als begriffliche Klammer für die formulierten Zielstellungen gewählt (Kap. 6.2.1). Auf Basis der empirischen und theoretischen Überlegungen (Kap. 5.2.3, 5.3, 5.4) können im Folgenden verschiedene Handlungen konkretisiert werden, die eine Artikulation und Reflexion des eigenen Denkens über den relevanten Themenbereich ermöglichen. Zur (Weiter-)Entwicklung einer pädagogischen Artikulations- und Reflexionsfähigkeit werden im Folgenden Lernhandlungen hinsichtlich vier unterscheidbarer Bereiche bzw. Phasen beschrieben: «Artikulation eigener Vorstellungen und Sprachfiguren» (Kap. 6.4.1.1), «Auseinandersetzung mit alternativen Sprachfiguren und Perspektiven» (Kap. 6.4.1.2), "Auseinandersetzung mit pädagogischen Perspektiven» (Kap. 6.4.1.3) sowie «Kriteriengeleitete Reflexion eigener Vorstellungen» (Kap. 6.4.1.4).

160 Öffentliche Wikis werden in diesem Zusammenhang als exemplarisches Beispiel für eine Sharing-Community verstanden (Kap. 4.3.3.3) sowie als Bestandteil einer partizipativen Medienkultur (Biermann et al. 2014). 


\subsubsection{Artikulation eigener Vorstellungen und Sprachfiguren}

Auf Basis der skizzierten Lernvoraussetzungen wird die Annahme vertreten, dass Studierende über erfahrungsbasierte Vorstellungen zu den Begriffen Lehren, Lernen, Didaktik und Bildung verfügen. Zugleich kann davon ausgegangen werden, dass sich die Studierenden diesen eigenen Vorstellungen nicht notwendigerweise explizit bewusst sind und diese nicht unmittelbar ins Bewusstsein gerufen werden können (Kap. 6.3.1). Gleichwohl haben die Vorstellungen Einfluss auf die Wahrnehmung entsprechender Seminare, so weist z. B. Blömeke (2004) auf die Filterwirkung entsprechender Vorstellungen hin (Kap. 6.3.1). Zum produktiven Umgang mit diesen Vorstellungen wird in Anlehnung an Blömeke (2004) und Horstkemper (2004) davon ausgegangen, dass es einer Bewusstwerdung sowie einer Versprachlichung der eigenen Vorstellungen bedarf (Kap. 5.2.3). Die Formulierung eigener Vorstellungen in Form von expliziten Metaphern bietet eine Möglichkeit, ein Nachdenken über die eigenen Vorstellungen und Überzeugungen anzuregen und über die Versprachlichung einen Zugang für Diskussionen und Rückmeldungen zu schaffen (Kap. 5.5, 5.4).

Im Rahmen der theoretischen Konstrukte wird in unterschiedlicher Weise davon ausgegangen, dass die Formulierung von Metaphern zur Explikation von impliziten Vorstellungen beiträgt. Saban et al. (2007) vertreten in dieser Hinsicht eine sehr optimistische Einschätzung der Möglichkeiten zur Formulierung expliziter Metaphern bzw. expliziter Vergleiche: «In this way we expected participants to make their implicit beliefs explicit» (Saban et al. 2007, 126). Trotz des gleichen methodischen Vorgehens zur Explikation von Metaphern beschreiben de Guerrero und Villamil $(2002,113)$ ihre Ergebnisse als «as revelatory of certain metaphors and metaphorical conceptualizations that are at the forefront of teachers' consciousness and readily accessible for externalization». Wenngleich die Autorinnen und Autoren der Studien die Beziehung zwischen den bewusst artikulierten Metaphern der Lernenden und ihren jeweiligen Überzeugungen unterschiedlich einschätzen, wird in beiden Untersuchungen das Reflexionspotenzial von explizit formulierten Metaphern hervorgehoben. In ähnlicher Weise diskutiert auch Marsch $(2009,107)$ den Forschungsstand. Als handlungsleitende Schlussfolgerung ihrer eigenen Untersuchung ist es für Marsch $(2009,107)$ vielversprechend, auf «die Explizierung der individuellen Metaphorik als Ansatzpunkt zur Entwicklung alternativer bzw. der Modifikation vorhandener Metaphern [zu setzen]». Für die Lehrerinnen- und Lehrerbildung schlussfolgert sie entsprechend, «dass Metaphern nicht vorgegeben werden können, sondern vom Individuum selbst konstruiert werden sollten» (ebd.).

Über die Formulierung von Metaphern wird das Ziel verfolgt, das eigene Denken über pädagogische Grundbegriffe und Zusammenhänge anzuregen. Im Fokus steht die Verschriftlichung bzw. Explikation der eigenen Vorstellungen über die Begriffe Lehren, Lernen und Bildung. Über die Verschriftlichung wird das eigene Denken zugänglich für Rückmeldungen von weiteren Personen sowie für eigene Reflexionen 
zu späteren Zeitpunkten. Die Formulierung von Metaphern stellt eine Voraussetzung dar, um im Verlauf einer Veranstaltung beurteilen zu können, inwiefern die eigenen (erfahrungsbasierten) Vorstellungen über Lehren und Lernen mit pädagogischer Theorie- und pädagogischen Konzepten übereinstimmen oder sich von diesen unterscheiden. So lassen sich potenzielle Lernhandlungen wie folgt konkretisieren:

- Die Studierenden versuchen sich über ihre eigenen Vorstellungen und Überzeugungen zu den zentralen Themen und Phänomenbereichen der Seminare bewusst zu werden.

- Die Studierenden versprachlichen ihre eigenen Vorstellungen in Form von expliziten Metaphern und verschriftlichen diese mithilfe unterschiedlicher medialer Repräsentationsformen.

\subsubsection{Auseinandersetzung mit alternativen Sprachfiguren und Perspektiven}

In der Annahme, dass die Versprachlichung und Verschriftlichung eigener expliziter Metaphern zum Nachdenken über die eigenen Vorstellungen anregt, wird weiterführend davon ausgegangen, dass eine Auseinandersetzung mit alternativen Sprachfiguren und Perspektiven hilfreich für die (Weiter-)Entwicklung einer pädagogischer Artikulations- und Reflexionsfähigkeit ist. Unter der Formulierung alternativer Sprachfiguren und Perspektiven werden zum einen weitere explizite Metaphern verstanden, welche alternative Ausdrucksweisen ähnlicher sowie konkurrierender Vorstellungen darstellen können. Zum anderen werden historische und aktuelle Entwürfe zur Didaktik sowie zu Lern- und Bildungstheorien als alternative Sprachfiguren und Perspektiven verstanden. Ferner wird davon ausgegangen, dass die Auseinandersetzung mit alternativen Sprachfiguren und Perspektiven in Gruppenarbeit in sprachlich und schriftlich vermittelter Form Lernprozesse anregen kann.

Die Auseinandersetzung mit Metaphern als sprachliche Ausdrucksmöglichkeiten in einem Seminar zu thematisieren, begründet sich vor dem Hintergrund der Überlegungen von Terhart (1999) und Gropengießer (2004) (Kap. 5.5.1, 5.3.3). So ist die «Erziehungswirklichkeit» für Terhart $(1999,155)$ als Gegenstand der Erziehungswissenschaft - und damit auch als Gegenstand der Didaktik sowie der Lern- und Bildungstheorien - in zweifacher Weise sprachlich vermittelt: «Erstens ist Erziehungshandeln selbst bereits zu einem sehr hohen Anteil sprachliches Handeln, und zweitens wird innerhalb der Alltagswelt immer schon über Erziehung gesprochen» (Terhart 1999, 155). Entsprechend beschreibt Terhart $(1999,156)$ «die Sprache bzw. das Sprechen» als eines der «zentralen Arbeitsmittel» von professionellen Pädagoginnen und Pädagogen. Die bewusste Auseinandersetzung mit Sprache und insbesondere mit alternativen metaphorischen Sprachfiguren kann in Anlehnung an Gropengießer (2004, 22) die Möglichkeit bieten, das eigene Denken und Handeln in Lehr- und Lernzusammenhängen «besser zur Sprache zu bringen, indem verschiedene Ausdrucksmöglichkeiten 
gewählt werden können». Die Fähigkeit, sich sprachlich bewusst ausdrücken und artikulieren zu können, bedingt die Möglichkeit, Vermittlungs- und Interaktionsprozesse für pädagogisches Handeln analysieren, begründen und bewerten zu können.

Die Auseinandersetzung mit Metaphern als versprachlichte Hinweise auf erfahrungsbasierte Vorstellungen der Studierenden begründet sich in Anlehnung an die Ausführungen von Pajares $(1992,320)$ zur Veränderung und Reflexion von Überzeugungen sowie in Anlehnung an Dann $(1989,252)$ zur Veränderung von Subjektiven Theorien von Lehrkräften. Trotz der jeweiligen unterschiedlichen theoretischen Konstrukte gehen beide Autoren davon aus, dass sich neues Wissen bzw. neue Vorstellungen - unabhängig davon, ob es sich um Subjektive Theorien oder didaktische Theorien handelt - in herausfordernden Situationen bewähren muss. So ist es für Pajares $(1992,320)$ unwahrscheinlich, dass Überzeugungen ersetzt werden, wenn sich diese nicht als unbefriedigend erweisen. In einer weiteren Negativbestimmung geht Pajares $(1992,320)$ davon aus, dass sich Überzeugungen kaum als unbefriedigend erweisen, wenn sie nicht infrage gestellt werden und man sie nicht in bestehende Konzepte integrieren kann. Für Dann $(1989,252)$ ist es bedeutsam, dass die Veränderung und Reflexion eigener Subjektiver Theorien «in praktisch relevanter Weise ablaufen, nämlich so, daß (sic) das neu entstehende Wissen besser zur Problembewältigung geeignet ist als das Alte». Für die vorliegende Arbeit wird in diesem Kontext das Reden bzw. Sprechen über die berufliche Tätigkeit von Lehrerinnen- und Lehrern als praktische Situation interpretiert. Auf Basis eigener praktischer Erfahrungen in der Arbeit mit Metaphern in Seminaren mit Lehramtsstudierenden hat sich zudem gezeigt, dass die schriftliche Auseinandersetzung mit alternativen Metaphern für Studierende Möglichkeiten bietet, die Implikationen und Grenzen alternativer Sprachfiguren diskutieren zu können ${ }^{161}$.

Eine Begründung dafür, warum die Auseinandersetzung mit alternativen Sprachfiguren und Perspektiven in Kleingruppen erfolgen sollte, bieten u. a. die bereits skizzierten empirischen Studien in realen Lehr-Lernsituationen (de Guerrero und Villamil 2000, 2002; Martinez et al. 2001). Beispielsweise berichten Martinez et al. (2001, 968) von dem kontinuierlichen Versuch Methoden zu etablieren, «which could give the

161 Exemplarisch veranschaulichen lässt sich diese Praxiserfahrung auf Basis einer Alltagsempirie an dem folgenden Textauszug einer Person, die an einem Seminar zur Diskussion explizit formulierter Metaphern teilnahm: «Allerdings empfinde ich nicht nur meine Metapher als unpassend. Einige weitere vorgestellte Beispiele waren in meinen Augen nicht ganz zutreffend. Besonders hervorheben möchte ich den Vergleich von Lemmingen, bei dem sich für mich ein grundsätzliches Problem ergibt. Im Fall der Lemminge geht man davon aus, dass der Lehrer vorangeht und hofft, dass ihm seine gehorsamen Schüler folgen. Dabei sollte ein Lehrer vielmehr wie ein Begleiter sein, der an der Seite der Schüler steht und sie bei ihrem (Lern)Weg unterstützt und bei auftretenden Schwierigkeiten hilft. Außerdem nimmt man bei dieser Metapher an, dass Lernende passiv sind und nicht selbstständig denken können, da sie ohne zu Hinterfragen (sic) in den Abgrund und damit in den sicheren Tod springen würden. Allerdings ist doch gerade dies eine der Aufgabe von Lehrenden, zu versuchen ihre Schüler zu mündigen Menschen, die selbstständig denken, zu erziehen. Was bringt den Schülern eine Lehrperson, die weiß wo es langgeht (oder auch nicht) und sie nicht lernen selbst den Weg zu finden?» (Seminar «Lehrende und Lernende im Unterricht», Wintersemester 2013/2014). 
participants access to their own tacit theories and promote reflection on their actions». Der Austausch von Ideen und Meinungen in kleinen Gruppen sowie die Auseinandersetzung mit Kontroversen erscheinen aus der Sicht von Martinez et al. (2001, 968) zur Erreichung ihrer Ziele als gut geeignet. Neben der Anregung von Diskussionen und kollaborativer Reflexionen verfolgten Martinez et al. $(2001,968)$ auch das Ziel, dass ihre Studierenden «become involved in group processes and to become familiar with methods of cooperative learning». In Anlehnung an Hinze (2008) werden insbesondere Formen der kollaborativen und kooperativen Zusammenarbeit in diesem Zusammenhang für sinnvoll erachtet. Diese Überlegungen erscheinen anschlussfähig an die formulierten Zielstellungen und es können die folgenden potenziellen Lernhandlungen konkretisiert werden:

- Die Studierenden vergleichen alternative Metaphern sowie die erhaltene Rückmeldungen mit ihren eigenen Vorstellungen und formulierten Metaphern.

- Die Studierenden setzen sich bewusst mit der Metaphorik unserer Sprache auseinander, insbesondere zum Sprechen über Lehr- und Lernsituationen.

- Die Studierenden diskutieren in Kleingruppen die Implikationen und Grenzen ihrer gewählten Metaphern und entwickeln in kollaborativer Zusammenarbeit eine Gruppenmetapher zur Artikulation ihrer gemeinsamen Vorstellungen.

\subsubsection{Auseinandersetzung mit pädagogischen Perspektiven}

Eine spezifische Form der Auseinandersetzung mit alternativen Sprachfiguren und Perspektiven sollte auch die Beschäftigung mit historischen und aktuellen Entwürfen zur Didaktik sowie zu Lern- und Bildungstheorien umfassen, denn diese wissenschaftlichen Perspektiven stellen eine Erweiterung der Subjektiven Theorien für mögliche Seminarteilnehmerinnen und -teilnehmer dar. In Anlehnung an Terhart (2009) beinhalten didaktische Modelle eine analytische sowie normative Dimension, welche als Kriterien zur Analyse und Bewertung pädagogischer Handlungen sowie zur Begründung eigener Planungen verwendet werden. Die analytische Dimension eines Modells kann mit Terhart $(2009,130)$ verstanden werden als

«eine vereinfachende, meistens graphische Darstellung von komplexen Sachverhalten und Zusammenhängen. Die Vereinfachung ist dabei bewusst gewählt, um zentrale Aspekte des komplexen Sachverhalts hervorzuheben, an denen man gerade besonders interessiert ist» (Terhart 2009, 130).

Neben der analytischen Dimension können entsprechende Modelle und Theorien, so Terhart $(2009,130)$, auch Orientierung in einem normativen Sinne bieten. Darunter versteht Terhart $(2009,130)$ «ein Vorbild, ein ideales Muster oder Beispiel, an dem sich andere orientieren sollten und können». In ähnlicher Weise unterscheidet Peterssen (2001) drei Funktionen didaktischer Modelle: (1.) orientierende Funktion, 
(2.) strukturierende Funktion und (3.) legitimierende Funktion. Die von Terhart (2009) und Peterssen (2001) formulierten Charakterisierungsvorschläge für didaktische Modelle sind hilfreich für eine strukturierte Auseinandersetzung mit einer pädagogischen und didaktischen Perspektive. Die Auseinandersetzung mit historischen und aktuellen Entwürfen zur Didaktik sowie zu Lern- und Bildungstheorien stellt die fachliche Grundlage dar, um Vermittlungs- und Interaktionsprozesse aus pädagogischer Perspektive zu analysieren, zu begründen und zu bewerten. Zur Erarbeitung dieser fachlichen Grundlage wird in Anlehnung an Hinze (2008) auch in diesem Zusammenhang davon ausgegangen, dass kollaborative und kooperative Formen der Zusammenarbeit vielversprechend sind. So lassen sich potenzielle Lernhandlungen wie folgt konkretisieren:

- Die Studierenden setzen sich lesend und denkend mit exemplarischen theoretischen Modellen zum Themen- und Gegenstandsbereich des Seminars auseinander.

- Die Studierenden recherchieren, diskutieren und dokumentieren kriteriengeleitet spezifische Aussagen didaktischer Modelle in kollaborativer und kooperativer Zusammenarbeit in Kleingruppen.

- Die Studierenden vergleichen ihre Recherche- und Dokumentationsergebnisse mit den Ergebnissen weiterer Studierender und reflektieren die Rückmeldungen von Studierenden und Lehrenden.

\subsubsection{Kriteriengeleitete Reflexion eigener Vorstellungen}

Die Formulierung einer kriteriengeleiteten Reflexion eigener Vorstellungen wird im Folgenden verstanden als analytische Betrachtung der zuvor verschriftlichten Metaphern als Ausdruck der eigenen Vorstellungen. Auf Basis der strukturierten Auseinandersetzung mit historischen und aktuellen Entwürfen zur Didaktik sowie zu Lern- und Bildungstheorien eröffnet sich die Möglichkeit, die eigenen Vorstellungen aus einer pädagogischen Perspektive betrachten zu können. Aus dieser Betrachtung heraus kann einerseits die Gemeinsamkeit der eigenen Perspektive mit einer pädagogischen Betrachtung identifiziert werden. Anderseits kann die Gegenüberstellung bzw. kriteriengeleitete Betrachtung der eigenen Vorstellungen mit pädagogischen Modellen zu Irritationen und kognitiven Konflikten führen (Duit 2010). In Anlehnung an die Erkenntnisse zu pädagogischen Überzeugungen (Pajares 1992, 320) und Subjektiven Theorien (Dann 1989, 252) wird davon ausgegangen, dass entsprechende Konflikte hilfreich sind. So formuliert Pajares $(1992,320)$ die Bedingung zur Veränderung und Reflexion von Überzeugungen wie folgt:

«A number of conditions must exist before students find anomalies uncomfortable enough to accommodate the conflicting information. First, they must understand that new information represents an anomaly. Second, they must 
believe that the information should be reconciled with existing beliefs. Third, they must want to reduce the inconsistencies among the beliefs. And last, efforts at assimilation must be perceived as unsuccessful. No small wonder, they argued, that conceptions are seldom weakened by anomalies. Students are generally unaware of their anomalies. In addition, if and when conceptual change takes place, newly acquired beliefs must be tested and found effective, or they risk being discarded» (Pajares 1992, 320).

In ähnlicher Weise beschreibt auch Dann $(1989,252)$ die Bedingungen zur Veränderung von Subjektiven Theorien. Dabei betont er die Bedeutung von Problemsituationen sowie eine notwendige Praxisrelevanz. Auf Basis dieser Überlegungen werden folgende Lernhandlungen als sinnvoll angenommen:

- Die Studierenden vergleichen die Aussagen theoretischer Modelle mit den eigenen Vorstellungen.

- Die Studierenden analysieren und dokumentieren die erstellten Gruppenmetaphern kriteriengeleitet bzw. aus einer spezifischen theoretischen Perspektive.

- Die Studierenden vergleichen ihre Ergebnisse mit weiteren studentischen Analysen anderer Metaphern sowie anderer Kriterien.

\subsubsection{Zusammenfassung}

Zusammenfassend wird die Annahme formuliert, dass die (Weiter-)Entwicklung pädagogischer Artikulations- und Reflexionsfähigkeit im Sinne der formulierten Zielstellungen (Kap. 6.2.1) sowie unter Berücksichtigung der angenommen Lernvoraussetzungen der Lehramtsstudierenden (Kap. 6.3.1) durch bestimmte Lehrhandlungen angeregt werden kann. Das anregende Potenzial dieser Lernhandlungen kann wie folgt zusammengefasst werden:

- Die Studierenden können sich durch die Artikulation von Metaphern ihrer eigenen (impliziten) Vorstellungen und häufig verwendeten Sprachfiguren hinsichtlich pädagogischer Zusammenhänge bewusstwerden und für andere nachvollziehbar dokumentieren.

- Die Studierenden können durch den Austausch und das kollaborative Schreiben mit anderen Studierenden die Implikationen und Grenzen bestimmter Metaphern für pädagogisches Handeln in Unterricht und Schule nachvollziehen und werden sich der Metaphorik pädagogischer Sprache bewusst.

- Die Studierenden können durch das Lesen und Diskutieren von Texten sowie das kollaborative Schreiben mit anderen Studierenden den analytischen und normativen Gehalt theoretischer Modelle für pädagogische Zusammenhänge nachvollziehen und für andere nachvollziehbar dokumentieren. 
- Die Studierenden können durch das theoriegeleitete Analysieren ausgewählter Metaphern eigene und fremde Vorstellungen über pädagogisches Handeln in Unterricht und Schule reflektieren und aus pädagogischer Perspektive begründen und bewerten.

\subsubsection{Teilhabe an einer partizipativen Medienkultur}

Die Formulierung der Teilhabe an einer partizipativen Medienkultur wurde als begriffliche Klammer für die formulierten Zielstellungen gewählt (Kap. 6.2.2). Diese Zielstellungen markieren spezifische Aspekte einer allgemeinen Medienkompetenz. In Anlehnung an die Ansätze zur handlungsorientierten Medienerziehung (Kap. 4.5.1), den Ausführungen zu relevanten Themenstellen sozialer Medien (Kap. 4.5.2) sowie den Erkenntnissen zu konkreten Spannungsfeldern beim Einsatz sozialer Medien in institutionellen Kontexten (Kap. 4.4.3) können im Folgenden verschiedene Handlungen konkretisiert werden, die eine Entwicklung der Fähigkeit zur Teilhabe an einer partizipativen Medienkultur anregen können. Zur Konkretisierung dieser Fähigkeit und zugehöriger Lernhandlungen wird in Anlehnung an das Strukturierungsmodell von Tulodziecki et al. $(2010,181)$ eine Differenzierung von vier Aufgabenbereichen vorgenommen: (a) «Gestalten und Veröffentlichen eigener Wikibeiträge» (Kap. 6.4.2.1), (b) «Durchschauen und Beurteilen von Bedingungen öffentlicher Wikis» (Kap. 6.4.2.2), (c) "Auswahl und Nutzen von sozialen Medien zur Kooperation und zum Lernen» (Kap. 6.4.2.3) sowie (d) «Verstehen und Bewerten von Medienbeiträgen» (Kap. 6.4.2.4).

\subsubsection{Gestalten und Veröffentlichen eigener Wikibeiträge}

Auf Basis der skizzierten Lernvoraussetzungen zum Lernen mit und über Medien (Kap. 6.3.2) wird davon ausgegangen, dass nur wenige Studierende über Erfahrungen zur Gestaltung und Verbreitung eigener Wikibeiträge verfügen. Während die Wikipedia als exemplarisches Wiki einer Vielzahl von Studierenden bekannt ist und regelmässig als Informationsquelle verwendet wird, verfügen nur sehr wenige Studierende über Erfahrungen in der aktiven Mitgestaltung und Teilhabe an öffentlichen Sharing-Plattformen im Allgemeinen sowie Wikis im Besonderen (Kap. 6.3.2). Dabei wurde u. a. von Herzig und Grafe (2007) vermutet, dass die fehlenden Erfahrungen auch ein Grund für die skeptischen Einstellungen gegenüber digitalen Medien sein können. In der Gestaltung und Veröffentlichung eigener Wikibeiträge wird das Potenzial gesehen, dass die Studierenden neue Erfahrungen im produktiven Umgang mit öffentlichen Wikis machen. Das Sammeln dieser Erfahrungen sowie das gemeinsame Diskutieren kann die Grundlage für eine erfahrungsfundierte Reflexion der eigenen Einstellungen gegenüber Wikis bzw. soziale Medien bilden. So können sowohl die eigenen Erfahrungen hinsichtlich der individuellen Nutzung als auch die Einsatzmöglichkeiten von 
Wikis in institutionellen Vermittlungssituationen hinterfragt werden. Zudem bietet der Aufgabenbereich zur aktiven Gestaltung und Veröffentlichung eigener Wikibeiträge einen Anlass zur (Weiter-)Entwicklung von Fähigkeiten zum kollaborativen und/oder kooperativen Schreiben von Texten als Ausdrucksform eigener Gedanken und Überlegungen, zur Entwicklung von technisch-instrumentellen Fähigkeiten zur Nutzung eines Wiki-Editors als technisches Hilfsmittel sowie Wissen über die Gestaltungs- und Strukturierungsmöglichkeiten von Wikibeiträgen.

In Anknüpfung an die Überlegungen von Tulodziecki et al. (2010, 250 ff.) kann der Aufgabenbereich zur aktiven Gestaltung und Veröffentlichung eigener Wikibeiträge in Form verschiedener Lernhandlungen weiter konkretisiert werden. Um einen Anlass zur Entwicklung der zuvor beschriebenen Fähigkeiten zu bieten und um die Reflexion der eigenen Einstellungen anzuregen, werden die folgenden Lernhandlungen als hilfreich angenommen:

- Studierende schreiben gemeinsam und individuell unterschiedliche Texte im Rahmen des Buchprojektes.

- Die Studierenden informieren sich über die Möglichkeiten der Textformatierung des Wiki-Editors sowie über die Veröffentlichung eigener Beiträge.

- Die Studierenden erstellen Wikibeiträge zur Veröffentlichung ihrer eigenen Überlegungen und Rechercheergebnisse.

- Die Studierenden beteiligen sich an der Mitgestaltung eines einheitlichen Layouts des Buchprojektes.

- Die Studierenden überarbeiten ihre eigenen Beiträge auf Basis von Rückmeldungen.

\subsubsection{Durchschauen und Beurteilen von Bedingungen der Wissensproduktion und Ver- breitung in öffentlichen Wikis}

In den skizzierten Lernvoraussetzungen wurde zum einen skizziert, dass die Studierenden kaum Erfahrungen mit einer aktiven (Mit-)Gestaltung von Beiträgen sowie der Teilhabe an Sharing-Communities haben (Kap. 6.3.2). Zum anderen ist es interessant, dass in der Akzeptanzanalyse zur Mediennutzung am Karlsruher Institut für Technologie (Grosch und Gidion 2011, 66 f.) die Wikipedia als bekanntestes Wiki den dritt höchsten Akzeptanzwert von 44 verschiedenen Anwendungen erzielen konnte. Offen ist dabei die Frage, über welches Wissen Lehramtsstudierende bezüglich der Bedingungen der Wissensproduktion und Verbreitung in öffentlichen Wikis verfügen. Über die Mitgestaltung eines gemeinsamen Buchprojektes wird die Möglichkeit gesehen, dass sich Studierende Kenntnisse über die technischen, rechtlichen und gesellschaftlichen Bedingungen öffentlicher Wissensproduktion in Sharing-Communities aneignen können. In Adaption der Überlegungen von Tulodziecki et al. (2010, 220) umfassen technische Bedingungen z. B. softwarespezifische Eigenschaften von Wikis 
als Webanwendung sowie Gestaltungsprinzipien zur Zusammenarbeit mit Wikis. Rechtliche Aspekte umfassen nach Tulodziecki et al. $(2010,228)$ z. B. das Urheberrechtsgesetz (UrhG) sowie das Bundesdatenschutzgesetz (BDSG). Die Erstellung eines eigenen Accounts sowie die Veröffentlichung eigener Beiträge und ggf. urheberrechtlich geschützten Materials stellen verschiedene Handlungen dar, um die entsprechenden rechtlichen Aspekte zu thematisieren. Gesellschaftliche Aspekte umfassen nach Tulodziecki et al. $(2010,308)$ u. a. Fragen «ob der Zugang für alle gesichert ist, kulturelle Vielfalt für sich angemessen abbildet und Innovation sowie Partizipation angeregt werden». In der Auseinandersetzung mit sozialen Medien wurde diesbezüglich bereits problematisiert, dass die technisch möglichen Partizipationspotenziale nur von sehr wenigen Menschen wahrgenommen werden (Kap. 4.5.2.1), auch was die Beteiligung an öffentlicher Wissensproduktion betrifft. In der Mitgestaltung eines Wikibooks wird die Möglichkeit gesehen, authentische Erfahrungen im Rahmen der Wikibook-Community bzw. in Auseinandersetzung mit Wikibookianerinnen und Wikibookianer ${ }^{162}$ machen zu können. Auf Basis dieser Erfahrungen können Prinzipien der Wissensproduktion und Verbreitung in öffentlichen Wikis in Anlehnung an Iske und Marotzki (2010) nachvollziehbar werden.

Für den Aufgabenbereich des Durchschauens und Beurteilens von Bedingungen der Wissensproduktion und Verbreitung in öffentlichen Wikis werden die folgenden Lernhandlungen als hilfreich angenommen, um Lernprozesse hinsichtlich der drei benannten Inhaltsdimensionen anzuregen:

- Die Studierenden informieren sich über die technischen Möglichkeiten zur Veränderung eigener Beiträge sowie zur Dokumentation dieser Veränderungen.

- Studierende nehmen über die technischen Möglichkeiten wahr, dass das gemeinsame Buchprojekt und/oder die von ihnen erstellten Beiträge durch Menschen ausserhalb des Seminars verändert und/oder kommentiert werden können.

- Die Studierenden diskutieren und reflektieren die Möglichkeiten und Probleme öffentlicher Partizipation für das konkrete Projekt sowie als allgemeines Prinzip öffentlicher Wikis.

- Studierende informieren sich über die Möglichkeiten und Grenzen, urheberrechtlich geschütztes Material unter freien Lizenzbestimmungen zu veröffentlichen.

\subsubsection{Auswahl und Nutzen von sozialen Medien zur Kooperation und zum Lernen}

Die Möglichkeiten zum Austausch sowie zur Kommunikation mit Freunden und Bekannten über soziale Online-Netzwerke sind gemäss der skizzierten Lernvoraussetzungen vielen Studierenden bekannt und werden von diesen regelmässig verwendet

162 Aktive Nutzerinnen und Nutzer der Plattform Wikibooks.org bezeichnen sich selbst als «Wikibookianerinnen bzw. Wikiboookianer». Eine Übersicht von Personen nach Wissensgebieten findet sich u. a. hier: https://de.wikibooks.org/wiki/ Wikibooks:Wikibookianer_nach_Wissensgebieten (01.01.2019). 
(Kap. 6.3.2). Über die Erstellung eines gemeinsamen Buchprojektes in Zusammenarbeit mit unterschiedlichen Arbeitsgruppen wird versucht einen Anlass zu schaffen, gemeinsam an verschiedenen Texten zu schreiben. Zudem sollen so die Möglichkeiten unterschiedlicher sozialer Medien erfahrbar werden, um in kollaborativer und kooperativer Zusammenarbeit digitale Produkte zu erstellen. In dieser Aufgabe wird das Potenzial gesehen, dass die Studierenden im Prozess ihre eigenen Erfahrungen zum Austausch und zur Kooperation mit und ohne soziale Medien zweckbezogen reflektieren, neue Handlungsmöglichkeiten kennenlernen und sich diesbezüglich instrumentelle Fähigkeiten aneignen können.

Die Auswahl und das Nutzen von sozialen Medien erfolgt im Rahmen des vorliegenden Projektes mit dem Fokus auf die zwei - von Tulodziecki et al. (2010, 246 f.) beschriebenen - Nutzungszusammenhänge «Lernen» sowie «Austausch und Kooperation». Als hilfreich erachtete Lernhandlungen sind die im Folgenden benannten Aktivitäten:

- Die Studierenden diskutieren und entscheiden in welcher Weise sie für die Erstellung der Wikibeiträge zusammenarbeiten möchten.

- Die Studierenden diskutieren in Gruppen die analogen und digitalen Möglichkeiten, sich im Rahmen des Buchprojektes auszutauschen und gemeinsam Texte zu erfassen.

- Die Studierenden erarbeiten gemeinsam Texte für das Buchprojekt und tauschen sich im Erstellungsprozess miteinander aus.

- Die Studierenden erfahren unterschiedliche Möglichkeiten der Rückmeldung zu ihren Textprodukten.

- Die Studierenden reflektieren die von ihnen getroffene Entscheidung zur Zusammenarbeit sowie zur Wahl der verwendeten Medien.

\subsubsection{Verstehen und Bewerten von Medienbeiträgen}

Die Wikipedia gilt als bekanntestes Wiki und stellt für viele Studierende eine Informationsquelle dar, welche von innen regelmässig verwendet wird (Kap. 6.3.2). Zugleich finden sich unterschiedliche Bewertungen der Vertrauenswürdigkeit von Wikipedia im Speziellen sowie Wikis im Allgemeinen (Kap. 6.3.2). Um Informationen von Wikis in Form von offenen Sharing-Communities kriteriengeleitet einschätzen zu können, eröffnet die Erstellung des geplanten Buchprojektes verschiedene Lernanlässe. Über die Darstellung unterschiedlicher Wissensformen ${ }^{163}$ hinsichtlich institutioneller Vermittlungsprozesse eröffnet sich u. a. die Möglichkeit, Lernprozesse über spezifische Darstellungs- und Gestaltungsformen sowie Gestaltungsarten von Texten anzuregen. Bei der Artikulation eigener Vorstellungen vom Lehren und Lernen mithilfe

163 Unterschieden werden kann mit Terhart (2009) in diesem Zusammenhang zwischen Alltagswissen, Metaphern und wissenschaftlichen Modelle der Didaktik. 
unterschiedlicher Medien (Kap. 6.4.1.1) können beispielsweise Unterschiede von Darstellungsformen thematisiert werden. Beim Vergleich unterschiedlicher Textsorten lassen sich zudem Differenzen zwischen der metaphorischen Darstellung von Erfahrungswissen (Kap. 6.4.1.1) sowie der kriteriengeleiteten Zusammenstellung wissenschaftlicher Erkenntnisse (Kap. 6.4.1.3) erkennen und diskutieren.

Der Aufgabenbereich «Verstehen und Bewerten von Medienbeiträgen» umfasst nach Tulodziecki et al. (2010, $269 \mathrm{ff}$.) ein sehr breites Spektrum an inhaltlichem Wissen über Medien. Über die Artikulation eigener Vorstellungen mithilfe unterschiedlicher Medienformate eröffnen sich unterschiedliche Lernanlässe. Für die gemeinsame Erstellung eines Buchprojektes stehen allerdings die "Gestaltungsmittel bei schriftlichen Texten» (Tulodziecki et al. 2010, 189 f.) sowie die «Gestaltungsmittel bei digitalen Medien» (Tulodziecki et al. 2010, 198) für unterschiedliche Textsorten im Fokus. Folgende Lernhandlungen werden als sinnvoll erachtet:

- Die Studierenden bringen ihre eigenen Vorstellungen mit dem bewusst gewählten Einsatz unterschiedlicher Medien zum Ausdruck.

- Die Studierenden diskutieren und vergleichen unterschiedliche mediale Möglichkeiten zur Artikulation ihrer Vorstellungen.

- Die Studierenden erarbeiten Aussagen über wissenschaftliche Modelle und dokumentieren diese nach Kriterien wissenschaftlichen Schreibens.

- Die Studierenden bewerten unterschiedliche Textsorten hinsichtlich ihrer Gestaltungsart und deren Angemessenheit in einer öffentlichen Wikigemeinschaft.

- Die Studierenden vergleichen die eigenen Beiträge mit den Beiträgen anderer hinsichtlich der gewählten Gestaltungsentscheidungen und diskutieren die Unterschiede.

\subsubsection{Zusammenfassung}

Zusammenfassend lässt sich die Annahme formulieren, dass die (Weiter-)Entwicklung der Fähigkeit zur Teilhabe an partizipativen Medienkulturen im Sinne der formulierten Zielstellungen (Kap. 6.2.2) sowie unter Berücksichtigung der angenommen Lernvoraussetzungen der Lehramtsstudierenden (Kap. 6.3.2) durch bestimmte Lernhandlungen angeregt werden kann. Das anregende Potenzial dieser Lernhandlungen kann wie folgt zusammengefasst werden:

- Die Studierenden können sich durch das (Mit-)Gestalten und Veröffentlichen eigener Wikibeiträge im Rahmen eines Buchprojektes instrumentelle Fähigkeiten zur produktiven und gestalterischen Nutzung von Wikis aneignen und reflektieren ihre eigenen Einstellungen gegenüber Wikis (im Unterricht).

- Die Studierenden können durch die Mitgestaltung eines öffentlichen Buchprojekts im Rahmen eines Wikis sowie der Prozessreflexion des Projektes technische, 
rechtliche und gesellschaftliche Bedingungen der Wissensproduktion und Verbreitung in öffentlichen Wikis durchschauen und beurteilen.

- Die Studierenden können durch das Erproben und Diskutieren unterschiedlicher sozialer Medien instrumentelle Fähigkeiten zum Kooperieren und Lernen erwerben sowie soziale Medien für spezifische Zwecke begründet auswählen.

- Die Studierenden können durch die bewusste Nutzung und Diskussion von Gestaltungsmitteln und unterschiedlichen Textsorten bei Wikibeiträgen die Fähigkeit entwickeln, Medienbeiträge zu verstehen und kriteriengeleitet zu bewerten.

\subsection{Lehrhandlungen}

Der Begriff Lehrhandlungen umfasst in Anlehnung an Grafe $(2008,143)$ alle Handlungen, welche auf die Anregung und Unterstützung von Lernaktivitäten gerichtet sind. Im Fokus stehen dafür die hochschuldidaktischen Handlungsebenen der «(Lern)Situationen» und der «(Lehr)Veranstaltungen» (Kap. 4.2.3). Das folgende Kapitel dient dem Zweck, den antizipierten Zusammenhang zwischen Zielvorstellungen (Kap. 6.2), Voraussetzungen der Lernenden (Kap. 6.3), sinnvoll erachteten Lernhandlungen (Kap. 6.4) sowie den eigenen Handlungsmöglichkeiten als Lehrender offen zu legen. Unter Berücksichtigung des hochschuldidaktischen Leitbildes «Wandel vom Lehren zum Lernen» (Kap. 4.4.1), des Forschungsstandes zum Lernen mit sozialen Medien (Kap. 4.4.2) sowie der Erkenntnisse zur Reflexion und Transformation erfahrungsbasierter Vorstellungen (Kap. 5.3.4) kann zunächst festgehalten werden, dass die verschiedenen Lernhandlungen nicht durch lehrzentrierte Darbietungen forciert werden können. Vielmehr wird davon ausgegangen, dass die im Folgenden skizzierten Gestaltungsentscheidungen Bedingungen schaffen können, um die zuvor beschrieben Lernhandlungen anzuregen und zu unterstützen ${ }^{164}$. Dafür werden drei Aspekte unterschieden: (a) «Produkt- und Projektorientierung» (Kap. 6.5.1), (b) «Kooperation, Kollaboration und Kommunikationsorientierung» (Kap. 6.5.2) sowie (c) «Eröffnung von Partizipationsmöglichkeiten und Erfahrungsorientierung» (Kap. 6.5.3).

\subsubsection{Produkt- und Projektorientierung}

In der gemeinsamen Gestaltung und Entwicklung eines Buches in einem öffentlichen Wiki wird die Möglichkeit gesehen, die zwei formulierten Zielstellungen verbinden zu können (Kap. 6.2). Die handlungsorientierte und gestalterische Erstellung eines entsprechenden Produktes ermöglicht vielfältige Erfahrungen und Lernprozesse (Kap.

164 Dabei wird nicht davon ausgegangen, dass die folgenden Lehrhandlungen in einer Wenn-dann-Kausalität zu den jeweiligen Lernhandlungen führen müssen. Beispielsweise problematisiert Holzkamp (1995) entsprechende Annahmen in Lehr- und Lernkontexten als «Lehr-Lern-Kurzschluss». Es kann jedoch begründet davon ausgegangen werden, dass die skizzierten Lehrhandlungen das Potenzial haben, die beschrieben Lernhandlungen anzuregen. 
4.5.1.2). Die didaktische Einbindung einer entsprechenden Produktorientierung erfolgt in Form eines Projekts. Das «Lernen in Projekten» kann in Anlehnung an Kerres $(2013,372)$ als eine Methode zur Gestaltung problemorientierter Lernangebote charakterisiert werden. Die Orientierung an dem Konzept der Problemorientierung bietet verschiedene Anschlussmöglichkeiten zu bisher skizzierten didaktischen Gestaltungsprinzipien (Kap. 4.5.1.2, 5.3) und kann nach Kerres $(2013,355)$ geeignet sein, «um komplexere Kompetenzen» zu vermitteln. Kerres $(2013,372)$ konkretisiert einen «problembasierten Ansatz des Projektlernens» hinsichtlich vier zentraler Eigenschaften. In der folgenden Beschreibung dieser Charakteristika werden die getroffenen Gestaltungsentscheidungen sowie die damit verbundenen Konsequenzen für das Lehrhandeln konkretisiert:

- Nach Kerres $(2013,372)$ ist «die Projektbearbeitung auf ein bestimmtes Lernziel ausgerichtet und in bestimmter Weise vorstrukturiert». Für das vorliegende Projekt wurden die (Weiter-)Entwicklung der pädagogischen Artikulations- und Reflexionsfähigkeit sowie die (Weiter-)Entwicklung der Fähigkeit zur Teilhabe an einer partizipativen Kultur als «Lernziele» formuliert (Kap. 6.2). Die Vorstrukturierung der Projektbearbeitung erfolgte in Anlehnung an die Überlegungen zum «Conceptual Change» sowie der Reflexion eigener Erfahrungen und Vorstellungen (Kap. 5.3). Die vier skizzierten Bereiche sinnvoller Lernhandlungen zur Entwicklung einer pädagogischen Artikulations- und Reflexionsfähigkeit (Kap. 6.4.1) dienten in diesem Zusammenhang als spezifische Reihenfolge zur Vorstrukturierung einer inhaltlichen Auseinandersetzung. Diese Reihenfolge wurde für die Strukturierung des Buchprojektes konkretisiert und führte zur Unterscheidung von drei Seminarphasen (Kap. 6.6.1).

- Die zweite Eigenschaft ist für Kerres $(2013,372)$, dass «sich der Lernprozess durch die Projektbearbeitung konstituiert (und nicht nur etwas zuvor Gelerntes im Projekt angewendet wird)». In dieser Anforderung zur Gestaltung von Lernsituationen lassen sich Bezüge zum Prinzip der Handlungsorientierung nach Tulodziecki et al. (2010) erkennen. In der Gestaltung und Veröffentlichung eines Wikibooks wird die Möglichkeit gesehen, dass die Vermittlung von Medienkompetenz im Sinne von Tulodziecki et al. (2010) «handlungsbezogen erfolgen» kann und Lernprozesse zur Teilhabe an einer partizipativen Kultur angeregt werden (Kap. 6.2.2). Auf pädagogisch-fachlicher Ebene wurde versucht dieser Anforderung durch die zuvor beschriebene Vorstrukturierung des Buchprojektes hinsichtlich förderlicher Lernhandlungen zur ersten Zieldimension (Kap. 6.2.1) gerecht zu werden.

- Als dritte Eigenschaft markiert Kerres (2013, 372), dass die «Lernenden mit zentralen Themen und Fragestellungen des Fachs konfrontiert werden (also nicht nur Teilaspekte einüben)». Diese Anforderung - analog zu den zwei skizzierten Aspekten - gilt es auf der Ebene des Planungshandelns als Lehrende zu berücksichtigen. Dies erfolgt zum einen durch die Themenstellung des Buchprojektes und 
deren Orientierung an pädagogischen Grundbegriffen. Zum anderen wird mit der Gestaltung des Buchprojektes ein exemplarischer Zugang zu aktuellen Themen und Fragen zur Teilhabe an partizipativen Medienkulturen eröffnet (Kap. 4.5.2).

- Die vierte Eigenschaft eines problembasierten Ansatzes nach Kerres $(2013,372)$ ist, dass «ein Artefakt mit Produktcharakter entsteht, so wie es in einem Praxisfeld außerhalb des Lernkontextes auch anzutreffen ist oder anzutreffen sein könnte». In ähnlicher Weise wird diese Anforderung von Schorb (2008) für sein Konzept der reflexiv-praktischen Medienarbeit formuliert: «Entscheidend ist [...] der Prozess der Herstellung eines medialen Produktes. In ihm erfolgt die Auseinandersetzung mit dem Gegenstandsbereich» (Schorb 2008, 83). Mit der Erstellung eines öffentlichen Wikibookprojektes wird im Sinne der Teilhabe an einer partizipativen Kultur (Kap. 6.2.2) ein Artefakt geschaffen, welches bereits im Prozess öffentlich zugänglich ist und mitgestaltet werden kann. Wird Sprache als «Werkzeug» pädagogischer Handlungen verstanden (Kap. 5.5.1) stellen auch die von Studierenden produzierten Texte ein Produkt dar, welche über den Lernkontext hinaus relevant sind. So müssen Lehrkräfte in der Lage sein, ihre eigenen Handlungen und Vorstellungen artikulieren und pädagogisch begründen zu können.

Relevante Aufgaben für Lehrende sind nach Kerres $(2013,373)$ u. a. die «Betreuung» und didaktische «Strukturierung des Lernangebotes». Die Betreuung der Studierenden umfasst u. a. Rückmeldungen für die Lernenden. Für den vorliegenden Projektkontext können und sollten diese Rückmeldungen für die unterschiedlichen Textprodukte formuliert werden. Ferner umfasst die Betreuung die «Reflexion und Auswertung» einzelner Phasen bzw. des gesamten Projektes (Kerres 2013, 372 f.). Die Unterstützung einer reflexiven Auseinandersetzung mit dem eigenen Handeln sowie der eigenen Perspektive begründet sich zudem durch Gestaltungsprinzipien einer handlungsorientierten Medienerziehung (Kap. 4.5.1.2) sowie durch die praktischen Überlegungen zum Umgang mit Beliefs bzw. erfahrungsbasierten Vorstellungen von Studierenden (Kap. 5.3). Ferner erwähnt Kerres $(2013,373)$ das Konzept «scaffolding», "als eine pädagogische Maßnahme, bei der das System so wenig Unterstützung wie möglich und zugleich so viel Unterstützung wie notwendig bietet». Für das vorliegende Projekt ist es daher sinnvoll, bei Bedarf spezifische Rückmeldungen für Arbeitsgruppen und individuelle Studierende zu geben.

Die didaktische «Strukturierung des Lernangebotes» (Kerres 2013, 372) umfasst - neben den bereits skizzierten Planungsentscheidungen - die Entwicklung eines komplexen Problems bzw. Arbeitsauftrages, der Vorbereitung von Lehr- und Lernmaterialien sowie die Bereitstellung angemessener Unterstützungsmassnahmen. Für die Formulierung einer entsprechenden Aufgabe betont Kerres $(2013,372)$, dass diese für die Lernenden als «Herausforderung» erlebt werden sollte. Zudem heben verschiedene Positionen hervor, so Kerres $(2013,372)$, dass «das Projekt ein echtes 
Vorhaben» sein sollte. Für die didaktische Strukturierung formuliert Kerres (2013, 373) den Hinweis, dass angemessene Prüfungsanforderungen zu stellen sind, welche sich am Projektcharakter orientieren.

\subsubsection{Kooperation, Kollaboration und Kommunikationsorientierung}

Eine zweite Möglichkeit zur Verknüpfung der zwei formulierten Zielstellungen (Kap. 6.2) sowie der zugehörigen Lernhandlungen (Kap. 6.4) wurde in der Schaffung kooperativer und kollaborativer Arbeitsphasen gesehen. Die Planung entsprechender Arbeitsphasen begründet sich $u$. a. hinsichtlich der Lern- und Bildungsvorstellungen im Zusammenhang mit sozialen Medien (Kap.4.4.2), den Möglichkeiten zur gemeinsamen Diskussion (und Reflexion) eigener Vorstellungen mithilfe von Metaphern (Kap. 5.4.2) sowie dem von Tulodziecki et al. (2010) benannten Prinzip der Kommunikationsorientierung (Kap. 4.5.1.2). Ferner sind laut Kerres (2013, 393) kooperative Lernszenarien häufig ein relevanter Bestandteil problemorientierter Methoden.

Lehrhandlungen umfassen in diesem Zusammenhang Planungs- und Gestaltungsentscheidungen zur Entwicklung kollaborativer Szenarien sowie pädagogischer Massnahmen zur Unterstützung und Betreuung der Studierenden. Die Planungsebene umfasst dabei u. a. die Gestaltung von Lernaufgaben sowie die Klärung praktischer Fragen zur Gruppengrösse sowie zur Zusammensetzung von Gruppen (Kerres 2013, 401 ff.). Neben den skizzierten Spannungsfeldern zum kooperativen und kollaborativen Lernen (Kap. 4.4.3) markiert Kerres $(2013,397)$ verschiedene «Hindernisse für kooperatives Lernen», welche auf der Planungsebene zu berücksichtigen sind. Wenngleich kooperatives und kollaboratives Lernen nicht aktiv «herstellbar» ist, kann Kollaboration durch unangemessene Lernaufgaben aktiv verhindert werden, wie Kerres $(2013$, 398) an vielfältigen Negativbeispielen zeigt. Im Rahmen des Projektes bedarf es zudem angemessener Unterstützungsmassnahmen bei der Gruppenbildung sowie bei der Betreuung der verschiedenen Arbeitsgruppen.

\subsubsection{Eröffnung von Partizipationsmöglichkeiten und Erfahrungsorientierung}

Die Eröffnung von Möglichkeiten zum partizipativen Lernen sowie die Orientierung an den Erfahrungen der Lernenden stellen zwei weitere Anforderungen an Lehrhandlungen dar, um die beschriebenen Lernhandlungen (Kap. 6.4) hinsichtlich der formulierten Zielstellungen (Kap. 6.2) unterstützen zu können. Diese Anforderungen begründen sich $u$. a. durch die Überlegungen zum Lernen mit und zum Lernen über soziale Medien in einer partizipativen Kultur (Kap. 4.3.2.2, 4.4.2.1, 4.5.2.1) durch das Gestaltungsprinzip der Erfahrungsorientierung einer handlungsorientierten Medienerziehung (Kap. 4.5.1.2), den Erkenntnissen zum Umgang mit erfahrungsbasierten Vorstellungen (Kap. 5.3) sowie den Untersuchungen zum Reflexionspotenzial von Metaphern (Kap. 5.4.3). 
Im Fokus dieser Lehrhandlungen stehen insbesondere Planungs- und Entwicklungsentscheidungen, um Studierenden Möglichkeiten zur Mitgestaltung sowie zur Artikulation eigener Erfahrungen zu eröffnen. Zur Orientierung unterscheidet Mayrberger (2013b, 169) neun "Stufen partizipative[n] Lernen[s] in formalen Bildungskontexten». Diese Stufen fasst Mayrberger (2013b, 169) in vier Formen zusammen: «Nicht-Partizipation» (Stufe 1-2), «Pseudo- oder Schein-Beteiligung; Vorstufen der Partizipation» (Stufe 3-5), «Partizipation» (Stufe 6-8), «über Partizipation hinaus; (volle) Autonomie» (Stufe 9). Die für dieses Projekt anzustrebende und zumindest phasenweise zu realisierende Form der «Partizipation» unterscheidet Mayrberger (2013b, 169) in drei Stufen: "Selbstbestimmung» ${ }^{165}$, «Mitbestimmung» ${ }^{166}$ und «Mitwirkung» ${ }^{167}$. Für die Entwicklung partizipativer Lernsituationen weist Mayrberger (2013a, 99) darauf hin, dass mit dem Partizipationsbegriff das (Macht)Verhältnis zwischen Lehrenden und Lernenden beschrieben wird. Für Lehrhandlungen bedeutet dies, bereit dazu zu sein, «Verantwortung für Entscheidungen bzw. Entscheidungsmacht abzugeben» (Mayrberger 2013a, 99). Zur Erreichung der formulierten Ziele sowie zur Anregung verschiedener Lernhandlungen wird es als sinnvoll erachtet, Möglichkeiten zur Mitbestimmung sowie zur Mitwirkung hinsichtlich der Gestaltung des Wikibooks (Kap. 6.4.2.1, 6.6.1.1, 6.6.1.2, 6.6.1.3) und hinsichtlich der Entwicklung von Metaphern als Ausdruck studentischer Vorstellungen (Kap. 6.4.1.1, 6.6.1.1) zu eröffnen.

\subsubsection{Zusammenfassung}

Zusammenfassend wird die Annahme vertreten, dass sich die als sinnvoll erachteten Lernhandlungen zur (Weiter-)Entwicklung einer pädagogischen Artikulations- und Reflexionsfähigkeit sowie zur (Weiter-)Entwicklung der Fähigkeit zur Teilhabe an einer partizipativen Medienkultur durch einen projektorientierten Ansatz zur gemeinsamen Gestaltung eines Wikibooks anregen und unterstützen lassen. Die relevanten Lehrhandlungen umfassen:

- Die Entwicklung eines Projektes mit einer authentischen und hinreichend herausfordernden Problem- und Aufgabenstellung, welches exemplarisch in der gemeinsamen Gestaltung eines Wikibooks realisiert wird.

165 «Selbstbestimmung» bedeutet für Mayrberger (2013b, 169), dass «der Lernprozess [...] nicht mit, sondern von Lernenden initiiert und [von] Lehrenden (partnerschaftlich) unterstützt [wird] (z. B. Inhalte, Ziele, Methoden etc.)».

166 Den Begriff der «Mitbestimmung» konkretisiert Mayrberger (2013b, 169) als «Beteiligungsrecht, das Lernende tatsächlich bei Entscheidungen mit einbezieht und ihnen Mitverantwortung überlässt. Die Idee für ein Lernvorhaben kommt von Lehrenden, doch alle Entscheidungen werden demokratisch mit den Lernenden getroffen (z. B. Methoden, Ablauf, Bewertungskriterien)».

167 Mayrberger (2013b, 169) versteht Mitwirkung als «indirekte Einflussnahme, um bei einer von Lehrenden gestalteten Lernsituation eigene Vorstellungen zu artikulieren, aber ohne bei der konkreten Umsetzung dabei zu sein (z. B. Feedbackrunden, Evaluation)». 
- Die Entwicklung von Kooperations- und Kollaborationsanlässen durch angemessene Aufgabenstellungen, genügend Freiräume zur Diskussion alternativer Sichtweisen sowie die Bereitstellung passender Unterstützungsmöglichkeiten.

- Die Eröffnung von Partizipationsmöglichkeiten zur Sammlung und Reflexion eigener Erfahrungen im Umgang mit sozialen Medien sowie einer inhaltlichen Auseinandersetzung mit einer pädagogischen Perspektive.

- Die Betreuung von Studierenden in Form von Beratung bei entstehenden Fragen sowie durch sinnvolle Rückmeldungen zu den erstellten Textprodukten der Studierenden.

\subsection{Entwurf von konzeptbezogenen Seminaren in der Pädagogik}

Auf Basis der präzisierten Zielvorstellungen (Kap. 6.2) und den formulierten Annahmen zu den Lernvoraussetzungen der Studierenden (Kap. 6.3) wird unter Berücksichtigung der theoretisch begründeten Überlegungen zu den Lernaktivitäten (Kap. 6.4) und dafür förderlichen Lehrhandlungen (Kap. 6.5) ein konkretes Seminarkonzept entwickelt. In Form eines projektorientierten Blended-Learning-Ansatzes wird zunächst ein Vorgehenskonzept bzw. eine Prozessplanung skizziert, in welchem zeitlichen Umfang die geplanten Lehrhandlungen realisiert werden sollen (Kap. 6.6.1). Daran anschliessend erfolgt die Auswahl und Begründung der medialen Kooperations- und Lernumgebungen in Form von Wikibooks und Moodle (Kap. 6.6.2) sowie eine Konkretisierung und Begründung der Inhalte am Beispiel des Seminars «Lehren und Lernen metaphorisch verstehen» (Kap. 6.6.3).

\subsubsection{Gestaltung und Begründung eines projektorientierten Blended-Learning- Konzeptes}

Mit dem Veranstaltungskonzept «Lehren, Lernen und Bildung metaphorisch verstehen? Ein kollaboratives Wikibookprojekt» wurde das Ziel verfolgt, über das gemeinsame Entwickeln eines Wikibooks, die Entwicklung fachlicher und medienbezogener Kompetenzen von (Lehramts-)Studierenden zu unterstützen und einen produktiven Beitrag für eine offene und partizipative Kultur zu leisten (Kap. 6.2). Zum Thema «Lehren, Lernen und Bildung» wurde dafür in verschiedenen Veranstaltungen in den Grundwissenschaften des Lehramtsstudiums (Pflichtmodule 1 \& 2, LAG 2009) an einem offenen Wikibook gearbeitet ${ }^{168}$. Die Entwicklung des Konzeptes begann im Wintersemester 2014/2015. Die erste Erprobung des Konzeptes sowie die Erarbeitung des Wikibooks erfolgte im Sommersemester 2015 in zwei Veranstaltungen. Zur

168 In Kooperation mit Nelson Gonçalves (ESE Viseu, Portugal) und Studierenden des Studiengangs «Artes Plásticas e Multimédia» wird über die Erstellung von Animationen für das Wikibook zudem ein fachund länderübergreifender Austausch zwischen Studierenden angestrebt. 
Realisierung wurde ein projektorientiertes Blended-Learning-Konzept mit drei Phasen entwickelt (Abb. 6.2):

1. Bewusstwerdung eigener Vorstellungen: Entwicklung von Metaphern zur Artikulation von (alltagsbezogenen) Vorstellungen der Studierenden zu pädagogischen Grundbegriffen und Handlungen. Verschriftlichung der in Gruppenarbeiten verdichteten Metaphern zu Wikiseiten und Videobotschaften.

2. Erarbeitung pädagogischer Perspektiven: Erarbeitung erziehungswissenschaftlicher Vorstellungen bzw. «Denkwerkzeuge» als Analysefolie für die selbst entwickelten expliziten Metaphern (aus Phase I) durch die Auseinandersetzung mit fachwissenschaftlicher Literatur. Erstellung von Wikiseiten und Diskussion im Seminar.

3. Kriteriengeleitete Reflexion eigener Vorstellungen: Erstellung von (individuellen) Analysen der eigenen Vorstellungen bzw. Metaphern (aus Phase I) mithilfe ausgewählter Kriterien/Kategorien der erarbeiteten erziehungswissenschaftlichen Vorstellungen bzw. «Denkwerkzeuge» (aus Phase II) in Form von eigenen Texten.

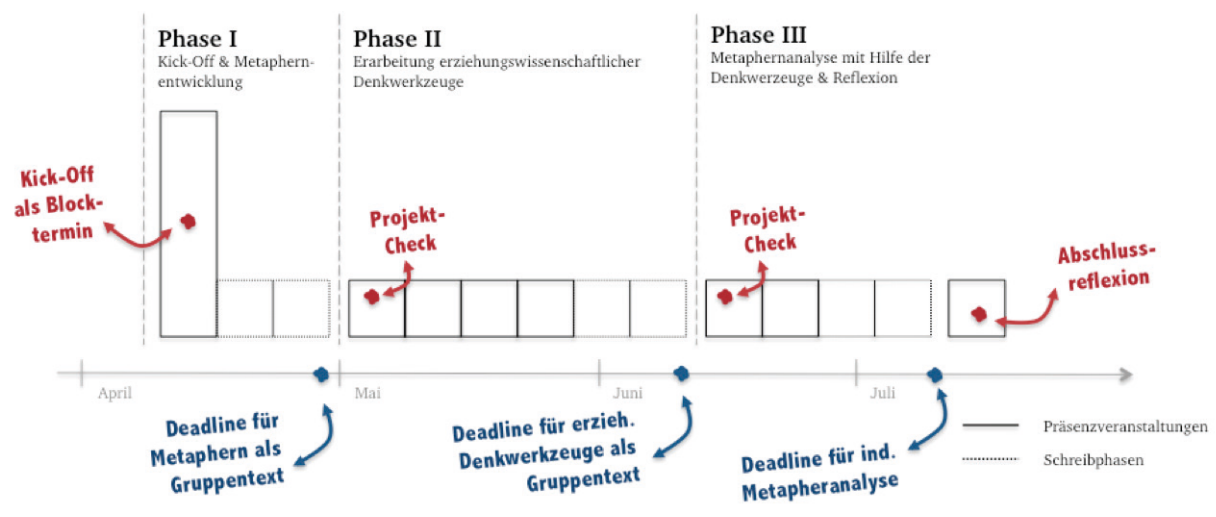

Abb. 6.2: Visualisierung der drei Veranstaltungsphasen (eigene Darstellung).

\subsubsection{Phase I: Entwicklung von Metaphern zur Bewusstwerdung eigener Vorstellungen}

Ziel der ersten Phase war es, über die Entwicklung von Metaphern die Vorstellungen der Studierenden über schul- und unterrichtsrelevante Aspekte sowie über ihre zukünftige Tätigkeit als Lehrende sichtbar und diese für eigene Reflexionen und Rückmeldungen von Dritten zugänglich zu machen. Somit war es möglich, (a) an die Lernvoraussetzungen der Studierenden anzuknüpfen und (b) das Spektrum unterschiedlicher Normalitätsvorstellungen hinsichtlich schul- und unterrichtsrelevanter Aspekte offenzulegen und aus pädagogischer Perspektive zu thematisieren (Kap. 6.4.1.1, 6.4.1.2). Ein möglicher Zugang zum Denken und zu den Vorstellungen der Studierenden ist die Arbeit mit Metaphern (Kap. 5.4). Eine zentrale Annahme des Konzeptes ist 
es, dass zum Verstehen und zur Erklärung abstrakter Begriffe und Konzepte (wie z. B. Didaktik oder Bildung) Erfahrungen aus anderen Bereichen herangezogen werden. Die Vorstellung "Lehren und Lernen ist wie Gehen und Reisen» ist ein typisches Beispiel für eine entsprechende Metapher. Zugleich wird über die jeweilige Interpretation und unterschiedliche Konkretisierungen sichtbar, wie Studierende z. B. die Rolle von Lehrenden verstehen: Die Vorstellung einer unterstützenden Begleitperson ist beispielsweise konträr zur Vorstellung des Lehrenden als direktive Führungsperson.

Zur Erarbeitung entsprechender Metaphern und Interpretationen wurde geplant, die Veranstaltungen mit einem Kick-off-Event über acht Stunden zu beginnen. Nach einer Erläuterung des Veranstaltungskonzeptes wurde in mehreren Schritten an der Entwicklung von Metaphern gearbeitet. Die Formulierung der Aufgabenstellung orientierte sich an den verschiedenen empirischen Untersuchungen ${ }^{169}$ sowie praktischen Erfahrungen ${ }^{170}$ zur Formulierung von expliziten Metaphern durch Studierende (Kap. 5.4.2). Die Aufgabenstellung bestand darin, ihre Vorstellungen vom Lehren und Lernen mithilfe einer Metapher zum Ausdruck zu bringen. Dafür bekamen die Studierende jeweils ein Arbeitsblatt (Abb. 6.3). Zur Bearbeitung standen den Studierenden 20 Minuten Zeit zur Verfügung. Die in diesem Zusammenhang entstanden Arbeitsprodukte der Studierenden werden im Folgenden als Ad-hoc-Metaphern bezeichnet.

Lehren und Lernen im Unterricht ist wie ...

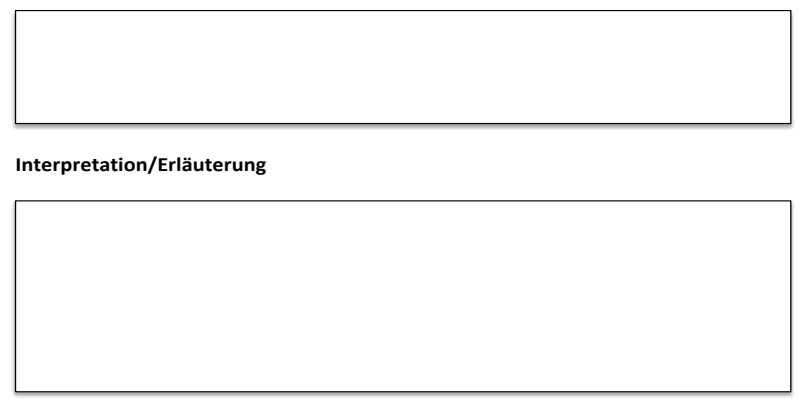

Abb. 6.3: Arbeitsblatt für Studierende zur Artikulation der eigenen Vorstellungen (eigene Darstellung).

169 Exemplarisch genannt werden können u. a. die Arbeiten von Arslan und Karatas (2015); Poom-Valickis et al. (2012); de Guerrero und Villamil (2000) und Saban et al. (2007).

170 Praktische Erfahrungen zur Arbeit mit Metaphern in (fach-)didaktischen Veranstaltungen, insbesondere als «Instrument» zur Artikulation der eigenen Vorstellungen wurden in der Arbeitsgruppe «Didaktik der Physik» (Universität Potsdam) sowie in der eigenen Lehre an der TU Darmstadt (u. a. im Seminar «Lehrende und Lehrende im Unterricht») gesammelt. Diese zeigen in Anlehnung an das Vorgehen von de Guerrero und Villamil (2000); Saban et al. (2007), dass Studierende in der Lage sind, explizite Metaphern zum Lehren und Lernen zu formulieren. Exemplarisch lässt sich dies an der Bearbeitung einer Reflexionsaufgabe in einem Seminar «Lehrende und Lernende im Unterricht» zeigen, bei dem Studierende mit ihren zu Beginn des Seminars entworfenen Metaphern auseinandersetzen sollten. Eine Person reflektiert diese Auseinandersetzung wie folgt: «Ich fand es sehr verwirrend, wie wenig ich mir über manche, sehr wichtige Begriffe bzw. Vorstellungen, Gedanken gemacht habe, wie als es um die eigene Vorstellung von Unterricht o. ä. ging. Ich hatte den Eindruck, dass meine Vorstellung eindeutig ist bis ich es verschriftlichen bzw. beschreiben sollte, mit einer Metapher, und bemerkte, dass ich es mir zunächst selbst klarmachen musste.» (Kommentar eines Feedbackbogens zum Seminar «Lehrende und Lernende im Unterricht», Wintersemester 2013/2014). 
Zur Diskussion der verschiedenen Ad-hoc-Metaphern wurden im Rahmen der Seminare tabellarische Übersichten zu den verwendete Quell- bzw. Bildbereichen erstellt und zur Sichtung und Interpretation während der Gruppenarbeiten ausgegeben sowie über Moodle als Arbeitsergebnisse dokumentiert. Die begründet ausgewählten Ad-hoc-Metaphern sollten in den folgenden «Think-Pair-Share»-Phasen jeweils als Diskussionsanlass dienen, um sich gemeinsam auf eine Metaphorik zu einigen bzw. gemeinsam eine neue Gruppenmetapher zu erstellen (Kap. 6.4.1.2). Die Diskussion und Auseinandersetzung erfolgte zur gestuften Entwicklung einer konsensfähigen Metapher als Grundlage für einen ersten Wikibeitrag. Die sich im Rahmen des Seminars gebildeten Gruppen erhielten die Aufgabenstellung, sich in ihrer Gruppe auf eine Metapher zu einigen, die besonders gut ihre Vorstellungen zum Lehren und Lernen zum Ausdruck bringt sowie Grenzen und Probleme der Metapher zu identifizieren ${ }^{171}$.

Nach einer zwei- bis dreistündigen Entwicklungsphase wurde geplant, die Metaphernentwürfe in Form von Flipcharts von Gruppen mit vier bis sechs Personen im Plenum zur Diskussion zu stellen und gegenseitig zu kommentieren. Über die Plenumsdiskussion sollte den Studierenden ein Anlass gegeben werden, dass eigene Denken zu versprachlichen und die gruppenspezifischen Vorstellungen mithilfe von Metaphern zum Ausdruck zu bringen. Durch die Plenumssituation sollten weitere Diskussions- und Austauschmöglichkeiten gegeben werden, denn durch die Konfrontation mit weiteren Perspektiven über die Arbeitsgruppe hinaus kann es möglich werden, die Grenzen und Probleme bestimmter Bildbereiche zu erkennen. Auf einer produktorientierten Ebene sollte die Diskussion auch konkrete Hinweise eröffnen, wie die bereits in Form von Flipcharts vorbereiteten Wikibeiträge verbessert und im Sinne von Kollaborationsprozessen schrittweise überarbeitet werden können (Kap. 6.5.2).

Nach einer Überarbeitungsphase sollte eine handlungs- und produktorientierte Gruppenarbeitsphase mit zwei Aufgabenstellungen folgen: Zum einen sollten Videobotschaften für Studierende in Portugal zur Animation der verschiedenen Metaphern erstellt werden. Zum anderen sollte mit der Verschriftlichung der eigenen Metaphern zur Erstellung eines Wikitextes begonnen werden (Kap. 6.4.2.1). Im Anschluss an das Kick-off-Event begann für die Studierenden eine zweiwöchige Schreibphase mit der Möglichkeit, individuelle Beratung und Feedback auf erstellte Textentwürfe einzufordern. Das (Vor-)Veröffentlichen von Textentwürfen sowie die Formulierung von Rückmeldungen erfolgte während der Schreibphase über Moodle. Die erste Phase endete mit der Veröffentlichung der ausformulierten Metaphern als Unterkapitel im Wikibook sowie einem abschliessenden Feedback (mit ggf. notwendigen Überarbeitungsvorschlägen).

171 Die Kombination individueller Aufgabenstellungen zu Beginn sowie die folgende Diskussion und Zusammenarbeit in Kleingruppen orientiert sich an dem Vorgehen von Martinez et al. (2001, 968). 


\subsubsection{Phase II: Erarbeitung pädagogischer Perspektiven als mögliche «Denkwerkzeuge»}

Zur Erarbeitung erziehungswissenschaftlicher "Denkwerkzeuge» ${ }^{172}$ lassen sich zunächst zwei Formen von Präsenzsitzungen ${ }^{173}$ unterscheiden. Im Rahmen des ersten Projekttreffens wurde zum einen das Ziel verfolgt, einen Rückblick auf die bisherigen Erfahrungen zur Erstellung des Wikibooks aufzuarbeiten. Zum anderen sollten Entscheidungen zum weiteren Vorgehen gemeinsam getroffen werden. Während die erste Sitzung zeitlich und inhaltlich vorstrukturiert wurde, eröffneten die folgenden Termine mehr Freiräume für studentische Gestaltungsideen (Kap. 6.5.3). Inhaltlich widmeten sich die Präsenzsitzungen der Diskussion erziehungswissenschaftlicher «Denkwerkzeuge» auf Basis des jeweiligen Bearbeitungsstandes der studentischen Arbeitsgruppen.

Das Ziel der zweiten Phase war es, pädagogisches und didaktisches Orientierungs- und Reflexionswissen in Gruppen zu erarbeiten, um eigene (und zukünftige) Lehr- und Lernerfahrungen denkend verarbeiten und pädagogisch analysierend beurteilen zu können (Kap. 6.4.1.3). Dafür sollten jeweils verschiedene Wiki-Artikel gestaltet und veröffentlicht werden (Kap. 6.4.2.1). Orientierungs- und Reflexionswissen werden in diesem Zusammenhang als grundlegende Kenntnisse von didaktischen Modellen sowie Lern- und Bildungstheorien verstanden (Kap. 6.6.3).

Zur Erarbeitung von Grundkenntnissen über die verschiedenen Modelle und Theorien konnten sich die Studierenden zunächst aus einer Vorauswahl für ein zu vertiefendes Thema entscheiden. Studierende, die das gleiche Thema wählten, bildeten in Phase II eine Expertinnen- und Expertengruppe. In Anlehnung an die JigsawMethode bzw. an ein Gruppenpuzzle wurden die Studierenden dafür gebeten, in den Expertinnen- und Expertengruppen mit jeweils neuen Gruppenmitgliedern zusammenzuarbeiten (Abb. 6.4). Ein entsprechendes Vorgehen ist die Voraussetzung dafür, dass in Phase III die zu Beginn erarbeiteten Metaphern aus verschiedenen theoretischen Perspektiven analysiert werden können. Die Unterstützung der Expertinnenund Expertengruppen (Kap. 6.5.2) erfolgte (a) über die Bereitstellung von Literaturempfehlungen und Überblickstexten sowie über eine (inhaltliche) Formatvorlage zur Strukturierung der Texte, (b) über die Anlegung von Gruppenforen auf Moodle zum Austausch von Entwurfstexten sowie zur Diskussion, (c) über die Durchführung regelmässiger Präsenzsitzungen zur Diskussion von (durch die Studierenden ausgewählten) Fachtexten oder bereits erstellten Wikitexten bzw. deren aktuellen Arbeitsstand

172 Mit der Metapher «Denkwerkzeug» soll darauf aufmerksam gemacht werden, dass eine Auseinandersetzung mit erziehungswissenschaftlichen Modellen und Theorien keinesfalls einem reinen Selbstzweck dienen soll. Vielmehr geht es darum (erziehungs-)wissenschaftliches Wissen als Möglichkeit zu verstehen, in einer neuen und systematischen Art und Weise über das Lehren und Lernen, die damit verbundenen professionellen Aufgaben sowie über das pädagogische Tätigkeitsfeld öffentlicher Bildungsinstitutionen, nachdenken zu können.

173 Die Begriffe «Präsenzsitzung», «Präsenzveranstaltung», «Präsenzseminar» und «Präsenztermin» werden weitgehend synonym verwendet. Sie dienen zur Bezeichnung einer zeitlich abgeschlossenen Lernsituation, die Lehrende und Lernende am gleichen Ort durchgeführt haben. 
sowie (d) durch individuelle Beratungs- und Feedbackmöglichkeiten im Rahmen der Schreibphasen. Im Gegensatz zur Phase I gab es zunächst regelmässige Präsenztreffen über vier bis fünf Wochen.

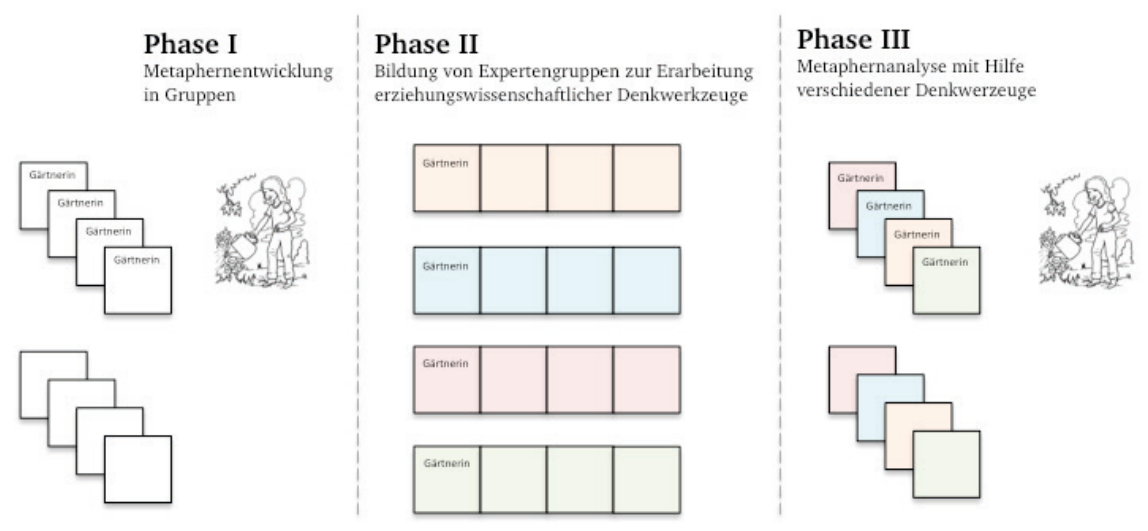

Abb. 6.4: Exemplarische Darstellung der Gruppenzusammensetzung am Beispiel einer Metapher für eine Gärtnerin (eigene Darstellung).

Analog zu Phase I wurde für die Studierenden eine zweiwöchige Schreibphase geplant. Sie hatten währenddessen die Möglichkeit, individuelle Beratung und Feedback auf erstellte Textentwürfe einzufordern (Kap. 6.4.2.1). Das (Vor-)Veröffentlichen von Textentwürfen sowie die Formulierung von Rückmeldungen konnte während der Schreibphase über Moodle oder über individuelle Sprechzeiten erfolgen. Phase II endete mit der Veröffentlichung des erarbeiteten erziehungswissenschaftlichen «Denkwerkzeugs» als Unterkapitel im Wikibook sowie einer Rückmeldung zur Qualität der Beiträge (mit ggf. notwendigen Überarbeitungsvorschlägen).

\subsubsection{Phase III: Kriteriengeleitete Reflexion eigener Vorstellungen}

In der abschliessenden Phase III der Seminare galt es, die in Form von Metaphern objektivierten Vorstellungen (aus Phase I) erneut zum Gegenstand zu machen. Mithilfe der erarbeiteten erziehungswissenschaftlichen «Denkwerkzeuge» wurde es dieser Phase möglich offenzulegen, inwiefern die Vorstellungen der Studierenden über schul- und unterrichtsrelevanten Aspekte sowie über ihre zukünftige Tätigkeit als Lehrende anschlussfähig an pädagogische Theorien und Modelle sind. Die Texte zu den Metaphern wurden dafür mit den Studierenden inhaltsanalytisch nach Kategorien der Denkwerkzeuge betrachtet (Kap. 6.4.1.4).

Zur Einführung in den Sinn und zum Vorgehen entsprechender Analysen wurden zu Beginn der Phase III Präsenzsitzungen geplant. In Betrachtung exemplarischer Metaphern als Demonstrationsmaterial wurden mithilfe eines ausgewählten erziehungswissenschaftlichen Denkwerkzeuges aus dem Wikibook in verschiedenen 
Varianten Analysen vorbereitet. Dabei wurden zum einen verschiedene Herausforderungen hinsichtlich des Vorgehens sichtbar (z. B. Festlegung eines Analysekriteriums), zum anderen konnten Stärken und Schwächen der bisher erarbeiteten Wikitexte, z. B. hinsichtlich der Konkretisierung der Metaphern oder der Nachvollziehbarkeit der «Denkwerkzeuge» thematisiert werden (Kap. 6.4.2.4). Anschliessend erfolgte die Erstellung der Analysen der Gruppenmetaphern in Schreibphasen mit individuellen Beratungsmöglichkeiten. Zur Dokumentation und Diskussion der Ergebnisse wurden Diskussionsforen zu den verschiedenen Metaphern bereitgestellt. Je nach Anzahl der Gruppenmitglieder wurde so eine Metapher mithilfe unterschiedlicher «Denkwerkzeuge» betrachtet.

Das Ziel war es, dass die Studierenden ihre durchgeführte Analyse als einen individuellen Beitrag im Wikibook formulieren und veröffentlichen. Mit der Aufgabenstellungen wurde auch das Ziel verfolgt, allen Studierenden handlungsorientierte Erfahrungen in der Nutzung und Gestaltung von Wikiseiten zu ermöglichen bzw. sie mit dem Wiki-Editor zu konfrontieren (Kap. 6.4.2.1). Zum Abschluss der dritten Phase erfolgte eine Reflexionssitzung zur Diskussion der gewonnenen Erkenntnisse sowie zur Einschätzung und Weiterentwicklung des Seminars. Zudem wurde geplant, allen erstellten Analysen ein individuelles Feedback zu formulieren.

\subsubsection{Auswahl und Begründung der medialen Kooperations- und Lernumgebung}

In Form eines Blended-Learning-Angebotes werden die formulierten Zielstellungen (Kap. 6.2) in unterschiedlicher Weise gefördert. Eine Unterstützung der fachlichen Kompetenzentwicklung erfolgt $u$. a. durch die Verpflichtung, das eigene Denken in regelmässigen Abständen schriftlich artikulieren und für das Wikibook aufbereiten zu müssen ${ }^{174}$ (Kap. 6.4.2.1). Durch regelmässiges Feedback über Moodle (in Form von Kommentaren und ausgefüllten Feedbackbögen) sowie der Möglichkeit, Rückmeldungen durch die Wikibookianerinnen und Wikibookianer einzuholen, kann eine Intensivierung der fachlichen Kompetenzentwicklung erfolgen (Kap. 6.5.1). Jenseits der verschiedenen Potenziale zum Lernen mit Medien bietet die Arbeit mit wikibooks.org die Chance zum Lernen über Medien. So nutzen viele Studierende Wikis wie Wikipedia selbstverständlich als Informationsquelle. Zugleich sind die wenigsten Studierenden weder mit den Grundprinzipien von Wikis vertraut noch nehmen sie an der Bearbeitung von Wikis teil. Durch die handlungsorientierte Auseinandersetzung und Mitgestaltung eines öffentlichen Wikis wird Studierenden die Möglichkeit eröffnet, einen «Blick hinter die Kulissen» zu erhalten. Ferner wird in der handlungsorientierten Vorgehensweise die Chance gesehen, Einblicke in die (kollaborative)

174 Gemäss der Studienordnung sind Studierende verpflichtet im Rahmen der Veranstaltung eine Studienleistung zu erbringen. Diese kann mit «bestanden» bzw. «nicht bestanden» bewertet werden. Im Rahmen des Konzeptes müssen die Studierenden die zuvor skizzierten Wikibeiträge in angemessener Qualität formulieren und veröffentlichen, um die Studienleistung zu bestehen. 
Wissensproduktion mithilfe von Wikis sowie in die Erstellung und Veröffentlichung eines Buches zu ermöglichen (Kap. 6.4.2.2). Die Wahl für die Verwendung von Wikibooks.org ${ }^{175}$ und Moodle als Learning-Management-System sowie die Frage nach der Sinnhaftigkeit zum Lernen mit digitalen Medien begründet sich dreifach: (a) durch die Chance, Lernende durch die Produktion von medialen Artefakten aktiver in die Lehr- und Lernveranstaltung einzubinden (Kap. 6.5.1), (b) durch das Potenzial, die Grenzen geschlossener Hochschulkurse durch die Beteiligung an einer offenen Gemeinschaft zu überwinden (Kap. 6.5.3) sowie (c) durch die Möglichkeit, verschiedene Formen der Kooperation, Kollaboration und des Feedbacks realisieren zu können. (Kap. 6.5.2).

- Mit der Formulierung «von der Rezeption zur Partizipation» beschreibt Ehlers $(2010,66)$ sehr optimistisch die im «E-Learning-2.0»-Diskurs vertretene Perspektive, Lernende als Impulsgeber ihrer eigenen Lernprozesse zu verstehen und sie als aktive (Ko-)Konstrukteure von Wissen und Materialien in Lehr- und Lernarrangements einzubinden (Kap. 4.4.1). Durch die gemeinsame Erstellung eines Buchprojektes mit Studierenden im Rahmen der offenen Wikigemeinschaft wikibooks.org wird diese Perspektive in Form eines projektorientierten Ansatzes aufgenommen (Kap. 6.5.1). In diesem Kontext beschreibt Anderson (2007, 33) die Chance, dass Lernende den Prozess des Lernens in einer produktiven Rolle (im Vergleich zu einer Konsumentenrolle) als überzeugender bzw. zwingender wahrnehmen (Kap. 4.4.2.1). Insbesondere der Aspekt, dass die produzierten Texte für Menschen jenseits der Lehr- und Lernveranstaltung einsehbar sind, kann für Studierende einen motivierenden Effekt haben. Wenngleich sich die eröffnenden Möglichkeiten für ein partizipatives und produktorientiertes Lernen durch die Bedingungen der Hochschullehre gerahmt sind, lohnt es sich (Kap. 4.4.3), die Chancen und Grenzen dieser Möglichkeiten näher zu bestimmen.

- Sozialen Medien im Allgemeinen sowie Wikis im Speziellen wird das Potenzial zugesprochen, die Grenzen geschlossener Hochschulkurse überwinden zu können (Grell und Rau 2011; Rau 2013). Guth und Petrucco (2009) beschreiben z. B. die Möglichkeit, in Lehrveranstaltungen über den Tellerrand zu schauen: «Social

175 Die Selbstdarstellung der Plattform lautet gemäss der Hauptseite von Wikibooks (2016) wie folgt: «Wikibooks - Die freie Bibliothek) für Lehr-, Fach- und Sachbücher wurde 2003 als Lehrbuchabteilung der Wikipedia eingerichtet. Seither haben viele Freiwillige 25.961 Buchkapitel in 713 Büchern geschrieben. Die 88 bereits fertigen Bücher sind im Buchkatalog zu finden. Auch viele Bücher, die nicht fertig sind und deshalb nicht im Buchkatalog stehen, können lesbar und informativ sein. Was bedeutet (frei)? Jeder darf alle diese Bücher frei nutzen - auch kommerziell, solange die Lizenzbestimmungen der CC-BY-SA-3.0 eingehalten werden. Doch nicht alle Arten von Büchern sind willkommen. Wikibooks ist eine Bibliothek nur für Lehr-, Sach- und Fachbücher. Unsere Lehrbücher sollen gesichertes Wissen widerspiegeln. Das heißt, die hier vermittelten Kenntnisse sind in ähnlicher Form in anderen tatsächlich publizierten Werken des jeweiligen Fachs bereits dargestellt. Allerdings können wir nicht für Fehlerfreiheit unserer Bücher garantieren: Die Bücher werden von vielen Autoren geschrieben, auch von NichtSpezialisten, und dabei können Fehler vorkommen. Vielleicht möchtest Du dich beteiligen und dein Wissen einbringen? Die ersten Schritte sind ganz einfach. Auf dem Wikibooks-Portal gibt es weitere Hilfestellungen sowie Möglichkeiten zur Beteiligung» (Wikibooks 2016). 
software tools give students the opportunity to interact with experts and novices alike on a global scale beyond planned classroom activities» (Guth und Petrucco 2009, 426). In öffentlichen Wikis wird dies u. a. daran deutlich, dass die Menschen (jenseits der eigenen Veranstaltungen) über die Diskussionsseite von WikiArtikeln inhaltliche Kontroversen führen. Guth und Petrucco (2009) betonen in diesem Kontext die Chance, dass die Kollaboration durch Social Software nicht ausserhalb des Vorlesungssaals oder des Seminarraums aufhört, «but rather flows over into personal lives and develops through the use of social software tools such as blogs, wikis, and forums» (Guth und Petrucco 2009, 426). So ermöglicht die Verwendung von wikibooks.org nicht nur die Chance, dass die Texte von Menschen gelesen werden, sondern dass diese auch kommentiert, kritisiert und überarbeitet werden. Entsprechende Erfahrungen können dazu führen, dass Studierende über die Auseinandersetzung mit den medialen Formaten sich auch jenseits der Veranstaltungen mit den Themen auseinandersetzen. Vor dem Hintergrund der skizzierten Lernvoraussetzungen (Kap. 6.3.2) und der Spannungsfelder institutioneller Lehre (Kap. 4.4.3.2) darf dieses motivierende Potenzial jedoch nicht überbewertet werden. Gleichwohl eröffnen sich durch die Arbeit mit einem offenen Wikibook vielfältige Anlässe zur handlungsorientierten Entwicklung medienpädagogischer Kompetenzen (Kap. 6.4.2).

- Mit dem Einsatz von Wikis und Moodle und den verbundenen neuen Inhaltsformen können Ideen und Gedankengänge Einzelner dokumentierbar artikuliert werden - wodurch neue Möglichkeiten der eigenen und wechselseitigen Referenzierung entstehen - und in Wikis und auf Moodle sichtbar geteilt werden (Kap. 4.4.2.2). Iske und Marotzki (2010) weisen darauf hin, dass durch diese Artikulation «Erfahrungen sowohl für den Autor wie auch für Dritte überhaupt erst sichtbar, adressierbar und referenzierbar und damit zum Ausgangspunkt individueller wie kollaborativer Nutzung ('sharing)) [werden]» (Iske und Marotzki 2010, 146). Kollaboration erfolgt in diesem Sinne im Kontext inhaltlicher Auseinandersetzung und basiert auf dem Teilen von Informationen, Erfahrungen und Erkenntnissen. Damit geht einerseits die Herausforderung einher, dass Studierende Wikis entsprechend kollaborativ nutzen müssen, anderseits eröffnet sich die Chance, dass für Studierende durch entsprechende Kooperationsformate neue Lern- und Bildungserfahrungen möglich werden (Kap. 6.4.1.2, 6.4.2.3). Zudem wird es möglich verschiedene Formen der Rückmeldung zu formulieren (Kap. 6.5.2) und deren Möglichkeiten und Grenzen im Spannungsfeld der institutionellen Rahmenbedingungen (Kap. 4.4.3.3) zu diskutieren. 


\subsubsection{Inhaltliche Schwerpunktsetzung und organisatorischer Rahmen}

Die inhaltliche Schwerpunktsetzung erfolgt unter Berücksichtigung der theoretischen und empirischen Erkenntnisse der Kapitel 4 und 5. Die inhaltliche Konzeption von Lehrveranstaltungen wird zudem durch Modulzuordnungen der TU Darmstadt und damit verbundenen curricularen Vorgaben gerahmt. Die Entwicklung des Konzeptes wurde für zwei Seminare im Studiengang «Lehramt an Gymnasien» (LaG) in den Grundwissenschaften ${ }^{176}$ der TU Darmstadt entwickelt (TU Darmstadt 2009a). Das angebotene Seminar «Lehren und Lernen metaphorisch verstehen? Ein kollaboratives Wikibookprojekt» war im Lehramtsstudium an der TU Darmstadt im Pflichtmodul «P2 Didaktik, Methodik und Medien» (TU Darmstadt 2009a, 3) verortet. Im Rahmen dieses Moduls entsprach das Seminar dem Modulbestandteil «P2.1: Allgemeine Didaktik (V2 oder S2)» (TU Darmstadt 2009b, 3). Das zweite Seminar «Bildung metaphorisch verstehen? Ein kollaboratives Wikibookprojekt» war im Pflichtmodul «P1: Grundlagen pädagogischen Denkens und Handelns» verortet (TU Darmstadt 2009a, 2). Das Seminar entsprach dem Modulbestandteil «P1.2: Bildungstheorie (V2 oder S2)» (TU Darmstadt 2009a, 2). Die vorgegebenen Inhalte und Kompetenzen waren anschlussfähig an die skizzierten Leitbilder der erziehungswissenschaftlichen Studienanteile im Lehramtsstudium (Kap. 5.2.1). Zugleich waren die Inhalte zur medienpädagogischen Professionalisierung von Lehramtsstudierenden im Rahmen der Studienordnung nicht verankert. Wie bereits im Rahmen der potenziellen Lernhandlungen skizziert (Kap. 6.4.2), wurden verschiedene Vorüberlegungen zur Anregung und Förderung entsprechender Lern- und Entwicklungsprozesse formuliert. Als inhaltliche Orientierungsrahmen dienten die zwei vorgestellten Themen- und Diskussionsfelder "Teilnahme und Teilhabe an einer partizipativen Kultur» (Kap. 4.5.2.1) und «Reflexion von Privatheit und informationeller Selbstbestimmung» (Kap. 4.5.2.2). Zudem wurde der Versuch unternommen, offen für die Erfahrungen der Studierenden im Prozess der Wikibookerstellung zu sein.

Die Konkretisierung relevanter Inhalte gemäss der Modulbeschreibungen kann exemplarisch für die Lehrveranstaltung «Lehren und Lernen metaphorisch verstehen» skizziert werden. Entsprechend der Modulbeschreibung steht die Auseinandersetzung mit didaktischen Modellen und lerntheoretischen Ansätze im Fokus der inhaltlichen Auseinandersetzung. Als exemplarische Schwerpunktsetzungen konnten die Studierende eigene Wünsche einbringen oder sich an Vorschlägen im Rahmen der Veranstaltung orientieren. Vorschläge für didaktische Modelle umfassten die «Bildungstheoretische Didaktik» und die kritisch-konstruktive Didaktik nach Klafki (1997), die Lehr-/Lerntheoretische Didaktik nach Heinmann (1962) und Schulz

176 Der Begriff der Grundwissenschaften bezeichnet an der TU Darmstadt die erziehungswissenschaftlichen Anteile im Lehramtsstudium (Horstkemper 2004) bzw. steht für die Bildungswissenschaften (KMK 2014). So soll das Studium Grundwissenschaften «den Studierenden als künftigen Lehrerinnen und Lehrern ein wissenschaftlich fundiertes und theoretisch reflektiertes Verständnis für die pädagogische Dimension ihrer beruflichen Tätigkeit vermitteln» (TU Darmstadt 2009a, 3). 
(2006), die kommunikative Didaktik nach Winkel (1997) sowie die konstruktivistische Didaktik nach Reich (2012). Die Auswahl der verschiedenen didaktischen Modelle als Vorschläge für inhaltliche Schwerpunktsetzungen der Studierenden erfolgte auf Basis didaktischer Lehrbücher. Berücksichtigt wurden u. a. die Bücher und Sammelwerke von Gudjons und Winkel (2006), Jank und Meyer (2008), Terhart (2009) und de Witt und Czerwionka (2007) sowie weitere Grundlagenliteratur von Kron et al. (2014), Klingberg (1990) und Peterssen (2001). Wenngleich die Praktikabilität didaktischer Modelle als Planungshilfen für den Unterricht in Frage gestellt wird (Wernke et al. 2015), sind sie im Sinne von Terhart (2009) und Peterssen (2001) hilfreich, um Unterricht in didaktisch strukturierter Weise betrachten und mit didaktischen Begriffen diskutieren und bewerten zu können ${ }^{177}$. Zudem scheinen die ausgewählten didaktischen Modelle Möglichkeiten zu eröffnen, diese wissenschaftlichen Konzepte - über die Betrachtung der jeweiligen Metaphorik - in Beziehung zu den eigenen Vorstellungen setzen zu können. Diese Möglichkeiten wurde in Ansätzen bereits bei den Ergebnissen von Peyer und Künzli (1999) deutlich (Kap. 5.5.1).

Um den Studierenden eine Vorbereitung auf die Veranstaltung sowie die inhaltliche Vertiefung zu ermöglichen, erfolgte eine ausführliche Beschreibung des Veranstaltungskonzeptes im Rahmen des Vorlesungsverzeichnisses. Für Studierende waren die Veranstaltungen unter den entsprechenden Titeln und gemäss ihrer modularen Verortung im Vorlesungsverzeichnis sichtbar (Anhang D.1.1).

\subsection{Zusammenfassung}

Mit dem vorliegenden Kapitel wurde der Entwurf eines Seminarkonzeptes für Pflichtveranstaltungen in den erziehungswissenschaftlichen Studienanteilen der Lehrerinnen- und Lehrerbildung vorgelegt. Der Entwurf wurde vor dem Hintergrund der problematisierten Praxis sowie unter Berücksichtigung des aufgearbeiteten Forschungsstandes (Kap. 4, 5) entwickelt und fokussiert die hochschuldidaktischen Handlungsebenen der «(Lehr)Veranstaltungen» sowie der «(Lern)Situationen» (Wildt 2002). Ausgehend von der Annahme, dass die Gestaltung der Praxis ein zentraler «Bestandteil des Forschungsprozesses und der Wissensgenerierung» (Tulodziecki et al. 2013, 227) im Kontext gestaltungs- und entwicklungsorientierter Bildungsforschung ist, stand die Darstellung und Begründung der getroffenen Gestaltungs- bzw. Entwicklungsentscheidungen im Fokus dieses Kapitels. Die Beschreibung erfolgte in Anlehnung an Tulodziecki et al. (2013) zunächst auf Konzeptebene und wurde abschliessend für spezifische Lehrveranstaltungen an der TU Darmstadt konkretisiert.

177 Die Auswahl möglicher Lerntheorien bzw. -paradigmen erfolgte mit Verweis auf Künkler (2011) sowie de Witt und Czerwionka (2007). Bildungstheoretische Ansätze und Überlegungen für die Veranstaltung «Bildung metaphorisch verstehen» wurden u. a. mit Verweis auf Klafki (1991) vorgestellt. 
Zu Beginn des Kapitels erfolgte eine methodische Verortung der vorgestellten Überlegungen als Bestandteil der Forschungsphase «Problematisierung und Entwurf» im Rahmen eines entwicklungsorientierten Bildungsforschungsprojektes (Kap. 6.1). Daran anschliessend wurden zunächst die Zielvorstellungen für ein Seminarkonzept in den erziehungswissenschaftlichen Studienanteilen expliziert und konkretisiert (Kap. 6.2) sowie Annahmen zu den (Lern-)Voraussetzungen der potenziellen Studierenden formuliert (Kap. 6.3). Die Formulierung der Zielstellung orientierte sich an den Leitbildern einer integrativen Medienbildung (Kap. 4.2.1). Demnach soll durch das Lernen mit Medien (1.) das fachliche Lernen unterstützt werden sowie (2.) ein Lernen über Medien angeregt werden. Das fachliche Lernen wurde als (Weiter-)Entwicklung der pädagogischen Artikulations- und Reflexionsfähigkeit der Studierenden konkretisiert (Kap. 6.2.1). Das Lernen über Medien wurde als (Weiter-) Entwicklung der Fähigkeit zur Teilhabe an partizipativen Medienkulturen spezifiziert (Kap. 6.2.2). Die Konkretisierung der formulierten Teilziele sowie deren Relevanz begründet sich durch die identifizierten Praxisprobleme sowie der skizzierten Forschungsperspektiven (Kap. 4, 5). Auf Basis verschiedener Untersuchungen wurde davon ausgegangen, dass Studierende zwar über eine gute technische Ausstattung, aber nur über wenig Erfahrungen mit der produktiven und gestalterischen Nutzung sozialer Medien verfügen (Kap. 6.3.2). Zudem wurde davon ausgegangen, dass Studierende über erfahrungsfundierte Vorstellungen über Unterricht, Lehren und Lernen verfügen, welche gemäss Blömeke (2004) als spezifische Lernvoraussetzungen betrachtet werden können (Kap. 6.3.1).

Im Folgenden wurden Annahmen zu potenziellen Lernhandlungen (Kap. 6.4) sowie förderlichen Lehrhandlungen (Kap. 6.5) formuliert. In Anlehnung an die Zielstellungen wurde zwischen zwei Lernzusammenhängen unterschieden: Lernen als (Weiter-)Entwicklung pädagogischer Artikulations- und Reflexionsfähigkeit (Kap. 6.4.1) sowie Lernen als (Weiter-)Entwicklung der Fähigkeit zur Teilhabe an einer partizipativen Medienkultur (Kap. 6.4.2). Die Gestaltung von Bedingungen zur Anregung und Unterstützung potenzieller Lernhandlungen erfolgte in Anlehnung an die skizzierten mediendidaktischen und medienerzieherischen Ansätze und Erkenntnisse (Kap. 4.4, 4.5). Drei Aspekte bzw. Prinzipien wurden hervorgehoben: (a) Lernen in Projekten und Problemorientierung (Kap. 6.5.1), (b) Kooperation, Kollaboration und Kommunikationsorientierung (Kap. 6.5.2) sowie (c) die Eröffnung von Partizipationsmöglichkeiten und Erfahrungsorientierung (Kap. 6.5.3). Auf Basis dieser Prinzipien wurde die Entscheidung getroffen, im Rahmen eines Seminars ein authentisches Projekt zur Mitgestaltung einer offenen Sharing-Community zu entwickeln, welches potenziell eKooperations- und Kollaborationsanlässe schafft und Partizipationsmöglichkeiten sowie neue Erfahrungen für die Studierenden eröffnet. 
Zum Abschluss des Kapitels wurde mit dem Entwurf von konzeptbezogenen Seminaren für die erziehungswissenschaftlichen Studienanteile (Kap. 6.6) eine Konkretisierung für die Rahmenbedingungen der TU Darmstadt vorgenommen und eine Prozessplanung zur Gestaltung und Begründung eines Blended-Learning-Konzeptes (Kap. 6.6.1) vorgelegt. Die Konkretisierung erfolgte für Seminare im Umfang von zwei Semesterwochenstunden und drei Creditpoints, welche in den Pflichtmodulen «P2: Didaktik, Methodik und Medien» (TU Darmstadt 2009b, 3) und «P1: Grundlagen pädagogischen Denkens und Handelns» (TU Darmstadt 2009b, 2) verortet waren. Das Konzept beinhaltet drei unterscheidbare Seminarphasen mit verschiedenen Lernsituationen. Zur Anregung von potenziellen Lernhandlungen zur (Weiter-)Entwicklung pädagogischer Artikulations- und Reflexionsfähigkeit (Kap. 6.4.1) wurden verschiedene Anlässe zur Artikulation und Reflexion der eigenen Vorstellungen in Form von individuellen und kollektiven Metaphern entwickelt. Zur Ermöglichung eines Lernens im Sinne einer (Weiter-)Entwicklung der Fähigkeit zur Teilhabe an einer partizipativen Medienkultur (Kap. 6.4.2) erfolgte die Mitgestaltung eines offenen Wikibooks (Kap. 6.6.2).

Der vorgelegte Entwurf kann mit den Worten von Sesink und Reinmann $(2015,75)$ als «eine in der Vorstellung vorweg genommene «besseres Praxis» beschrieben werden. Diese Vorstellung gilt es nach Sesink und Reinmann $(2015,75)$ im Rahmen eines entwicklungsorientierten Bildungsforschungsprojektes «auf ihre Realisierungsmöglichkeiten hin» zu erproben. Diese Erprobung wird im folgenden Kapitel Experimentelle Praxis (Kap. 7) aus der Perspektive des Lehrenden reflektiert und mithilfe wissenschaftlicher Methoden empirisch analysiert. Auf Basis einer Auswertung und Neuperspektivierung (Kap. 8) der empirischen Ergebnisse werden zum Abschluss der ersten Iteration bzw. zum Beginn der zweiten Iteration des Projektes verschiedene Modifikationen des Entwurfs vorgenommen (Kap. 9). 


\section{Experimentelle Praxis}

\subsection{Methodische Verortung}

Die zweite Phase einer entwicklungsorientierten Bildungsforschung bezeichnen Sesink und Reinmann $(2015,75)$ als «Durchführung eines Entwicklungsprojekts: Experimentelle Praxis» (Abb. 7.1). Das erste Ziel dieser Phase ist die Erprobung des in der ersten Phase entwickelten Entwurfs einer neuen, besseren Praxis. Das zweite Ziel ist die systematische empirische Untersuchung und Reflexion der «zu erprobenden Praxis» (Sesink und Reinmann 2015, 80). Mit dem vorliegenden Kapitel werden die Praxisreflexionen aus Lehrendenperspektive sowie die Ergebnisse der empirischen Analysen für die Erprobung im Sommersemester 2015 dokumentiert. Sesink und Reinmann $(2015,80)$ formulieren diesbezüglich den Anspruch, diese Phase «als offener Erfahrungs- und Lernprozess aller Beteiligten zu konzipieren». Im Rahmen dieses Prozesses geht es für Sesink und Reinmann $(2015,80)$ sowohl darum die «Folgen» der zu erprobenden Praxis genau zu beobachten» als auch die «Dimension der Innovation» systematisch zu erfassen bzw. eine Aufmerksamkeit für Unerwartetes zu schaffen.

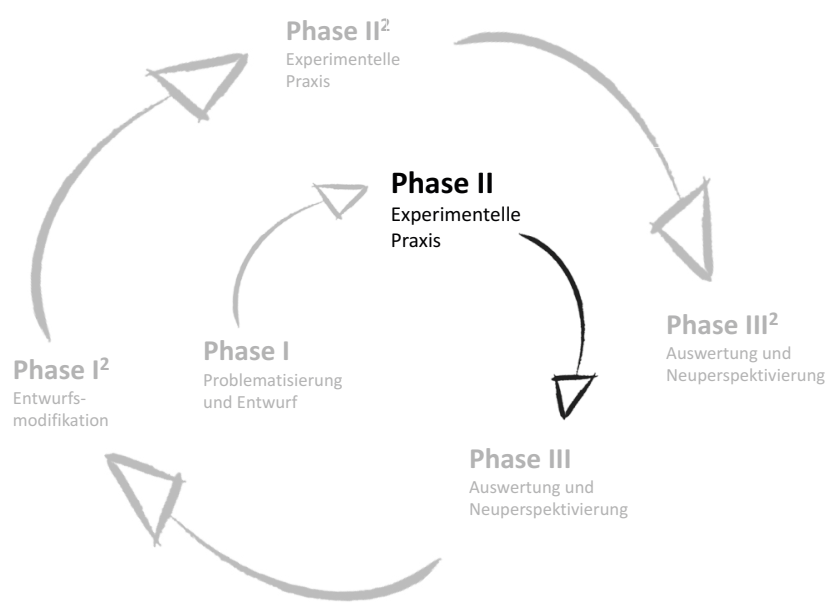

Abb. 7.1: Verortung des Kapitels im Phasenmodell der entwicklungsorientierten Bildungsforschung.

Die Konzeption der Erprobung sowie deren forschungsmethodische Analyse erfolgt durch eine Kombination unterschiedlicher Methoden zur Datenerhebung und -auswertung (Kap. 3). Die Dokumentation und Reflexion der Praxiserfahrungen aus der Perspektive des Lehrenden erfolgt in Anlehnung an Sesink und Reinmann (2015, 82) auf Basis konkreter Arbeitsprodukte, dokumentierter Prozessabläufe und aufgezeichneter Sprachmemos (Kap. 7.2). Zur Dokumentation der wissenschaftlichen 
Analyse erfolgt zunächst eine Darstellung der verwendeten Datenquellen sowie eine soziodemografische Verortung der Forschungspartnerinnen und -partner ${ }^{178}$.

Die Auswertung der unterschiedlichen Daten erfolgte mithilfe verschiedener Methoden, u. a. qualitative Inhaltsanalysen, Metaphernanalysen sowie Verfahren der deskriptiven Statistik (Kap. 7.3). Die Berücksichtigung der verschiedenen Daten und Methoden erfolgte mit dem Ziel, dem von Tulodziecki et al. $(2013,229)$ formulierten Prozessstandard zur «Verknüpfung unterschiedlicher Vorgehensweisen bei der Datenerfassung und Auswertung» gerecht zu werden. Im Rahmen dieses Kapitels erfolgt die Darstellung der Ergebnisse für die verschiedenen Datenquellen. Es wird zwischen den Ergebnissen der Interviews (Kap. 7.4.1), der Veranstaltungsevaluation (Kap. 7.4.2) der Analyse der studentischen Metaphern (Kap. 7.4.3) sowie der Wikibookstatistiken (Kap. 7.4.4) unterschieden. Die vergleichende Betrachtung der einzelnen Ergebnisse wird im folgenden Kapitel «Auswertung und Neuperspektivierung» diskutiert (Kap. 8).

\subsection{Erprobung des Entwurfs als Prozessreflexion}

Auf Basis des geplanten Seminarkonzeptes (Kap. 6.6) erfolgte im Sommersemester 2015 an der TU Darmstadt die erste Erprobung (Kap. 3.1.2). Die Dokumentation der Praxiserfahrungen erfolgt aus der Perspektive des Lehrenden. Um diesen Perspektivwechsel im Rahmen des folgenden Kapitels auch sprachlich zum Ausdruck zu bringen, erfolgt die Darstellung der Erfahrungen überwiegend in der «lch-Perspektive». Die Realisierung der Seminare erfolgte mit Unterstützung der studentischen Tutorin Sophie Schaper, welche im Folgenden ebenfalls als Lehrende bezeichnet wird. Die Erprobung des Entwurfs wird chronologisch dargestellt. Die Rekonstruktion des Verlaufs basiert auf der Auswertung der aufgezeichneten Sprachmemos (Kap. 3.3.1), der Dokumentation der Veranstaltung über Moodle (Anhang D.1) sowie der erstellten Beiträge bei Wikibooks.org (Anhang E).

\subsubsection{Beschreibung der Seminargruppen}

Die Beschreibung der Seminargruppen basiert auf zwei Momentaufnahmen: (1) der Anzahl der bestätigten Teilnehmerinnen und Teilnehmer zu Beginn des Semesters über TUCaN ${ }^{179}$ sowie (2.) die Anzahl der bestätigten Anmeldezahlen zum Ende des Semesters. Die Präsentation und Beschreibung dieser Momentaufnahme erfolgt mit dem Ziel, einen ersten Überblick über die Seminargrösse und die Studierenden zu ermöglichen. Diese Momentaufnahme kann jedoch nicht den Anspruch erfüllen, die

178 Dies erfolgt für die durchgeführten Interviews sowie für die Evaluation.

179 TUCaN ist das Webportal des Campus-Management-Systems an der Technischen Universität Darmstadt. Online zugänglich unter https://www.tucan.tu-darmstadt.de/ (01.01.2019). 
Anzahl der Teilnehmerinnen und Teilnehmer im Seminar über das gesamte Semester abzubilden. Die Grenze begründet sich u. a. vor dem Hintergrund, dass an der TU Darmstadt keine Anwesenheitspflicht besteht und Studierende auch während des Semesters sich wieder von Veranstaltungen abmelden können. Daher wird auch in der Beschreibung der einzelnen Seminarphasen angegeben, wie viele Studierende an der Gestaltung der verschiedenen Arbeitsprodukte beteiligt waren.

Für das Seminar «Lehren und Lernen metaphorisch verstehen? Ein kollaboratives Wikibookprojekt» wurde zu Beginn des Sommersemesters 2015 die Anmeldung für 50 Studierenden über TUCaN bestätigt ${ }^{180}$. Zum Ende des Semesters waren noch 41 Studierende angemeldet (Tab. 7.1). 59\% der Teilnehmerinnen und Teilnehmer studierten im Studiengang Pädagogik (Bachelor of Arts) und 41\% der Teilnehmerinnen und Teilnehmer waren im Studiengang Lehramt an Gymnasien (1. Staatsexamen) eingeschrieben. Bezogen auf die Fachsemester konnte die Seminargruppe als heterogen beschrieben werden. Ein vielfältiges Bild zeigte sich auch bei Betrachtung der Fächer und Fächerkombinationen der Lehramtsstudierenden. Nur die Fächerkombinationen Chemie (LaG 2005) und Biologie (LaG 2008) sowie Deutsch (LaG 2013) und Philosophie/Ethik (LaG 2013) wurden von jeweils zwei Studierenden belegt. In Betrachtung der verschiedenen Fächer zeigte sich ein Gleichgewicht zwischen MINT ${ }^{181}$ Fächern $(n=21)$ und gesellschafts- und geisteswissenschaftliche Fächern $(n=18)^{182}$. Die am häufigsten studierten Fächer ${ }^{183}$ der Lehramtsstudierenden waren Biologie $(n=7)$, Philosophie/Ethik ( $n=7)$ und Mathematik $(n=6)$.

\begin{tabular}{|l|l|l|l|l|}
\hline Anzahl TN & Studiengang & FS (M) & min. FS & max. FS \\
\hline 17 & LAG (1. Staatsexamen) & 5,6 & 2 & 16 \\
\hline 24 & Pädagogik (BA) & 4,9 & 4 & 12 \\
\hline 41 & Gesamt & 5,2 & 2 & 16 \\
\hline
\end{tabular}

Tab. 7.1: Überblick über die Anzahl der Studierenden der Veranstaltung «Lehren und Lernen metaphorisch verstehen» nach Studiengang und Fachsemester (FS).

Für das Seminar «Bildung metaphorisch verstehen? Ein kollaboratives Wikibookprojekt» wurde zu Beginn des Sommersemesters die Anmeldung von 28 Studierenden des Lehramtes an Gymnasien (1. Staatsexamen) bestätigt. Zum Ende des Semesters

180 Die maximale Anzahl der Teilnehmerinnen und Teilnehmer für Seminarangebote von wissenschaftlichen Mitarbeiterinnen und Mitarbeiter lag im Institut für Allgemeine Pädagogik und Berufspädagogik im Sommersemester 2015 bei 50 Studierenden. Vor Beginn der Vorlesungszeit wird, sofern notwendig, über ein vom Studienbüro verwaltetes Losverfahren ausgewählt, welche Studierenden an den Veranstaltungen teilnehmen können.

181 MINT ist eine Abkürzung und setzt sich aus den Anfangsbuchstaben der Studienfachbereiche Mathematik, Informatik, Naturwissenschaften und Technik zusammen.

182 Die Anzahl von 39 verschiedenen ergibt sich dadurch, dass 12 Lehramtsstudierende jeweils zwei Hauptfächer und fünf Studierende jeweils drei Hauptfächer studierten.

183 Die vollständige Liste der studierten Fächer der Lehramtsstudierenden, sortiert nach Anzahl, lautet: Biologie $(n=7)$, Philosophie/Ethik $(n=7)$, Mathematik $(n=6)$, Deutsch $(n=5)$, Chemie $(n=4)$, Informatik $(n=3)$, Sport $(n=2)$, Politik und Wirtschaft $(n=2)$, Geschichte $(n=2)$ und Physik $(n=1)$. 
waren noch 16 Studierende angemeldet (Tab. 7.2). Hinsichtlich der Fachsemester der Studierenden wies die Seminargruppe ein breites Spektrum auf, welches vom 2. Fachsemester bis zum 12. Fachsemester reicht. Es zeigte sich ebenfalls ein vielfältiges Bild bei der Betrachtung der Fächer und Fächerkombinationen der Lehramtsstudierenden. Bei Betrachtung der verschiedenen Fächer fanden sich mehr gesellschafts- und geisteswissenschaftliche Studienfächer $(n=24)$ im Vergleich zu MINT-Fächern $(n=15)$ in der Seminargruppe ${ }^{184}$. Die häufigsten Fächer ${ }^{185}$ der Lehramtsstudierenden waren Philosophie/Ethik ( $n=7)$, Geschichte $(n=6)$ und Mathematik $(n=5)$.

\begin{tabular}{|l|l|l|l|l|}
\hline Anzahl TN & Studiengang & FS (M) & min. FS & max. FS \\
\hline 16 & LAG (1. Staatsexamen) & 4,1 & 1 & 12 \\
\hline
\end{tabular}

Tab. 7.2: Überblick über die Anzahl der Studierenden der Veranstaltung «Bildung metaphorisch verstehen» nach Studiengang und Fachsemester (FS).

Beide Seminargruppen waren gekennzeichnet durch eine Heterogenität hinsichtlich der gewählten Fächerkombinationen der Lehramtsstudierenden sowie der Anzahl an Fachsemester. Damit verbunden sind unterschiedliche Erfahrungen in den jeweiligen Fachdisziplinen hinsichtlich der Verwendung digitaler Medien zum Studium. Ferner unterscheidet sich die zeitliche Distanz zur eigenen Schulerfahrung zwischen verschiedenen Seminarteilnehmerinnen und -teilnehmer innerhalb der Gruppen.

\subsubsection{Phase I: Vorstellungen sichtbar machen}

Die Reflexion meiner Erfahrungen als Lehrender bei der praktischen Erprobung des Konzeptes in den zwei Seminaren «Lehren und Lernen metaphorisch verstehen» und «Bildung metaphorisch verstehen» wird im Folgenden für die erste Seminarphase hinsichtlich drei gewählter Schwerpunkte dargestellt: (1.) Durchführung und Erfahrungen des Kick-off-Termins, (2.) Materialien und Interaktionen über Moodle sowie (3.) Erleben und Thematisierung von Interaktionen mit Mitgliedern der Wikicommunity.

184 Die Anzahl der 39 verschiedenen Fächern ergibt sich dadurch, dass elf Lehramtsstudierende jeweils mit zwei Hauptfächern, vier Studierende mit jeweils drei Hauptfächern und eine weitere Person mit vier Hauptfächern angezeigt wurde.

185 Die vollständige Liste der studierten Fächer der Lehramtsstudierenden, sortiert nach Anzahl, lautet: Philosophie/Ethik $(n=7)$, Geschichte $(n=6)$, Mathematik $(n=5)$, Deutsch $(n=4)$, Informatik $(n=4)$, Politik und Wirtschaft $(n=4)$ Sport $(n=3)$, Chemie $(n=2)$, Biologie $(n=2)$ und Physik $(n=2)$. 


\subsubsection{Durchführung und Erfahrungen des Kick-off-Termins}

Der Seminarauftakt erfolgte in Form eines Blocktermins über acht Zeitstunden für jedes Seminar ${ }^{186}$. Die Veranstaltungen begannen mit einem 38-44 minütigen Vortrag zur Einführung in die Projektidee, im Rahmen eines Lehr- und Forschungsprojektes gemeinsam ein Wikibook zu schreiben. Diese Einführung ${ }^{187}$ umfasste (1.) die Vorstellung und Erläuterung des Projektes Wikibooks.org, (2.) des thematischen Schwerpunktes der Veranstaltung im Bezug zu den jeweiligen Studienordnungen, (3.) die studiengangspezifischen Leistungsanforderungen, (4.) des Ablaufs der Veranstaltung in Form des Phasenkonzepts und (5.) die Vorstellung der Tagesplanung in Form von drei Abschnitten (Warm-Up, Metaphernentwicklung, Diskussion und Produktion). Im Anschluss an die Einführung und eine Diskussion offener Fragen erfolgte als «WarmUp» eine Vorstellungsrunde aller Seminarbeteiligten und die erste Annäherung an Metaphern zum Lehren und Lernen (bzw. zu Bildung). Dafür erhielten die Studierenden ein Arbeitsblatt zur Vervollständigung des Vergleiches «Lehren und Lernen im Unterricht ist wie...» sowie zur Formulierung einer zugehörigen Erläuterung bzw. Interpretation der Metaphern (Abb. 6.3, Kap. 6.6.1.1). Der Zeitrahmen zur Bearbeitung betrug 20 Minuten. Im Anschluss an die Bearbeitung der Aufgaben hatten die Studierenden die erste Pause des Blocktermins. Für das Seminar «Lehren und Lernen metaphorisch verstehen» wurden 22 Arbeitsblätter abgegeben. Für das Seminar «Bildung metaphorisch verstehen» wurden zwölf Arbeitsblätter eingereicht.

Im Fokus der zweiten Phase stand zum einen die Anregung von Diskussionen zu den zuvor formulierten Vorstellungen sowie die Konfrontation mit alternativen Sichtweisen und Perspektiven. Zum anderen wurde das Ziel verfolgt, dass sich Studierende in kleinen Gruppen auf eine gemeinsame Metapher verständigen, die ihren Vorstellungen vom Lehren und Lernen bzw. von Bildung entspricht. Zur Vorbereitung der Seminarphase wurde die erste Pause von den Lehrenden genutzt, um die erstellten Ad-hoc-Metaphern der Studierenden für die zweite Phase der Metaphernentwicklung während des Blocktermins aufzubereiten. Dafür wurden jeweils die Bildbereiche der Ad-hoc-Metaphern in einer Tabelle zusammengestellt (Abb. 7.2). Die Erläuterung bzw. die Interpretation der Ad-hoc-Metapher wurde nicht übernommen. Stattdessen konnten die Studierenden in einem Freifeld jeweils individuelle Interpretationen notieren. Bei der Erstellung der Tabelle wurden ähnlich formulierte Bildbereiche ${ }^{188}$

186 Der Seminarauftakt der Veranstaltung «Lehren und Lernen metaphorisch verstehen erfolgte am Samstag, 11. April 2015. Der Seminarauftakt der Veranstaltung «Bildung metaphorisch verstehen» erfolgte am Samstag, 18. April 2015.

187 Dieser Einführungsvortrag wurde als Audioaufnahme jeweils aufgenommen und den Studierenden über Moodle zur Verfügung gestellt.

188 Der Begriff «Bildbereich» wird synonym zum Begriff «Quellbereich» (Schmitt 2017, 39) verwendet. In Anlehnung an die im Kapitel 5.3.3 vorgestellte Definition bezeichnet der Bild- bzw. Quellbereich die wörtliche Bedeutung eine Metapher. Der Begriff «Zielbereich» markiert den (häufig abstrakten) Bestandteil einer Metapher, auf den die wörtliche Bedeutung des konkreten Bildbereichs «übertragen» wird. 
zusammengefasst. Für das Seminar «Lehren und Lernen metaphorisch verstehen?» beinhaltete die tabellarische Übersicht 21 unterschiedlich formulierte Bildbereiche. Für das Seminar «Bildung metaphorisch verstehen?» beinhaltete die Übersichtstabelle 12 verschiedene Bildbereiche.
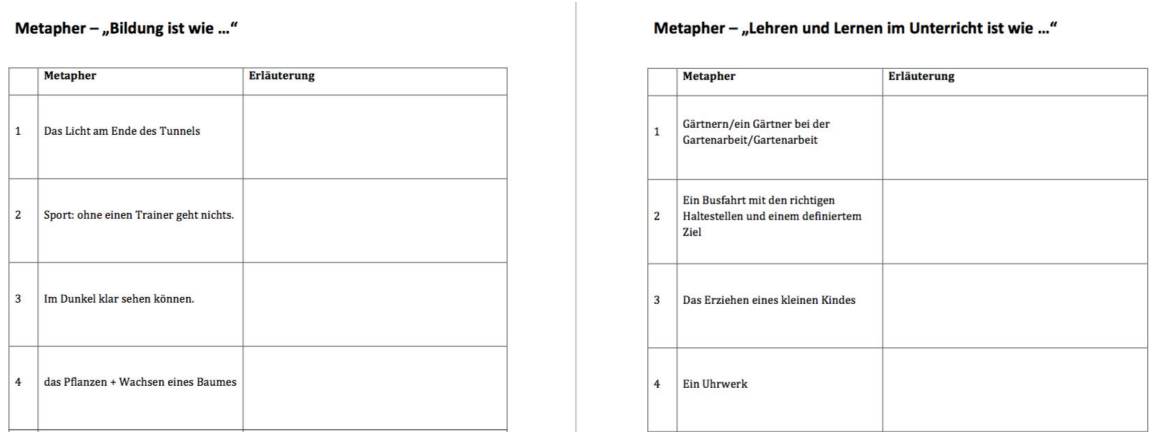

Abb. 7.2: Screenshot der Bildbereichsübersichten aus den Seminare «Bildung metaphorisch verstehen» (links) Lehren und Lernen metaphorisch verstehen» (rechts).

In Form einer adaptierten Think-Pair-Share Methode (Kap. 6.6.1.1) entstanden häufig lebhafte Diskussionen über die unterschiedlichen Interpretationen. Darauf aufbauend wurden in kleinen Gruppen Plakate zur Darstellung einer Gruppenmetapher für die Diskussion während der dritten Seminarphase entwickelt (Abb. 7.3). Die Lehrenden beschränkten sich in dieser Phase auf die Beachtung des zeitlichen Rahmens und die Beantwortung von studentischen Nachfragen. Zudem versuchten wir durch spezifischen Nachfragen zu den Interpretationen der Studierenden verschiedene Impulse und Anregungen in die Diskussionen der Arbeitsgruppen einzubringen.

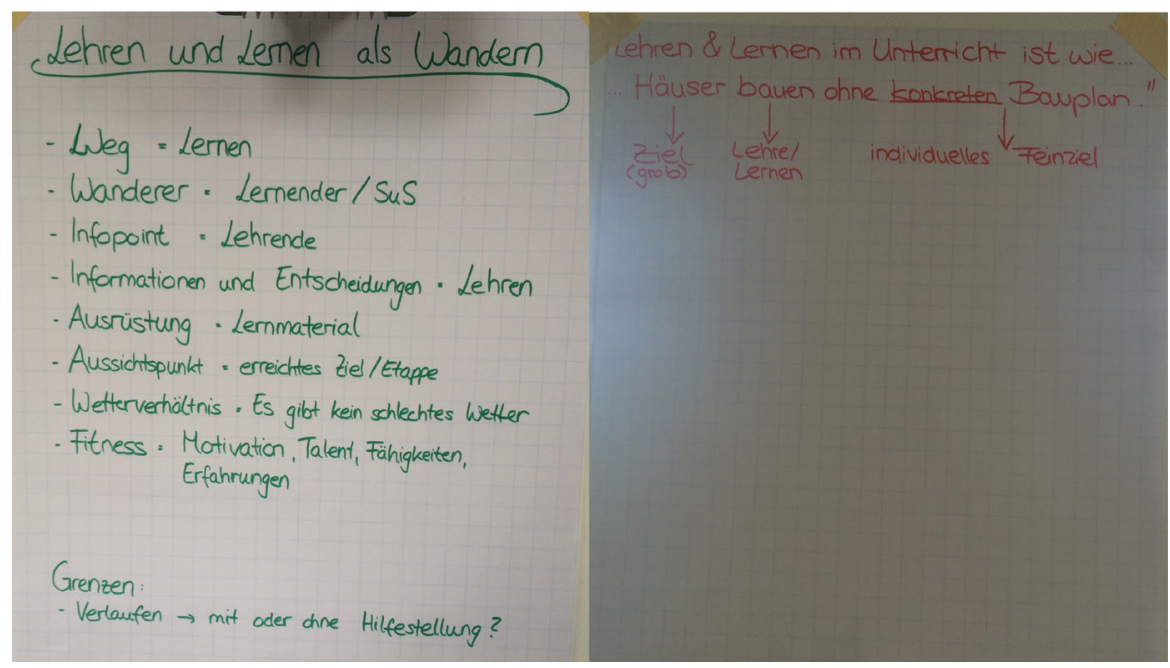

Abb. 7.3: Exemplarische Zwischenergebnisse zum Entwurf einer Gruppenmetapher im Seminar «Lehren und Lernen metaphorisch verstehen?» vom 11.04.2015. 
Die dritte Phase des Blockstermins wurde als «Diskussion und Produktion» bezeichnet. Die Phase begann mit einer Plenumsdiskussion der entwickelten Metaphern (Abb. 7.3). Die Aufgabenstellung für die studentischen Arbeitsgruppen bestand darin, als Gruppe ihre jeweilige Gruppenmetapher zu erläutern bzw. ihr Verständnis im Plenum zu erklären. Für die jeweils nicht präsentierenden Arbeitsgruppen wurde die Aufgabe formuliert, die Metaphern der anderen Arbeitsgruppen kritisch zu hinterfragen (Kap. 6.6). Dabei wurden insbesondere die Grenzen verschiedener Sprachbilder besprochen und diskutiert. Nach der Plenumsdiskussion überarbeiteten die Studierenden Aspekte ihrer Gruppenmetapher und widmeten sich der Produktion einer Videobotschaft für Studierende in Portugal sowie der Erstellung des Wikibeitrages. Die Bearbeitung der zwei Aufgaben erfolgte in den studentischen Arbeitsgruppen in unterschiedlicher Geschwindigkeit sowie in unterschiedlicher Intensität. Die anwesenden studentischen Arbeitsgruppen entschieden sich jeweils dazu, mit der Formulierung der Videoschaft zu beginnen und konnten diese auch im Rahmen des Blocktermins abschliessen. Zur Unterstützung der Studierenden wurde ihnen zunächst eine Vorlage für die Formulierung eines englischen Skripts zur Verfügung gestellt, auf dessen Grundlage sie ihre Videobotschaft formulieren konnten. Diese sollte die Grundlage für die Erstellung einer Animation ihrer Metaphern darstellen. Die Aufnahme der Videobotschaft wurde durch die studentische Tutorin unterstützt. Die studentischen Arbeitsgruppen begannen im Anschluss mit der Verschriftlichung der Metaphern. Das Schreiben erfolgte zunächst in verschiedenen Textverarbeitungsprogrammen.

Bei der Beratung der studentischen Arbeitsgruppen eröffnete sich durch die Nachfragen und Kommentare von Studierenden auch die Frage für mich als Lehrender und Forscher, inwiefern die entwickelten Gruppenmetaphern Rückschlüsse auf die individuellen Vorstellungen ermöglichen. Am Beispiel eines Sprachmemos lässt sich dies verdeutlichen:

«Es geht um eine Erfahrung im Rahmen der Präsenzsitzung und zwar ist Folgendes passiert. Jemand hatte gefragt, inwiefern (..) was sozusagen im Text erwartet wird und ob das jetzt noch konkreter gefasst werden muss und analytischer. Ich meinte, naja es muss deutlich werden, dass das eure Vorstellungen vom Lehren ist. Da hat [die Person] gelacht und meinte, naja [sie] weiss nicht so genau ob das jeweils die Vorstellung ist vom Lehren und Lernen» (Sprachmemo 003, 0:22-0:42 min, 17.04.2015).

Die Reaktion dieser Person war für mich der Anlass darüber nachzudenken, inwiefern die adressierten Ziele erreicht werden können. Es zeigte sich, dass in den Gruppenarbeiten über die Diskussion der unterschiedlichen Ad-hoc-Metaphern ein Austausch über unterschiedliche Aspekte des Lehrens und Lernens stattfand bzw. darüber, welche Aspekte für die verschiedenen Studierenden jeweils relevant waren. 
Inwiefern die formulierten Gruppenmetaphern konkrete Objektivationen der Vorstellungen der individuellen Studierenden darstellen bzw. in welchen Anteilen Studierende die aus ihrer jeweiligen Sicht relevanten Aspekte und Facetten von Lehren und Lernen in der Gruppenmetapher zum Ausdruck bringen können, erschien mir aufgrund der Reaktion der Studierenden allerdings fragwürdig. In einem Sprachmemo findet sich dazu folgende Einschätzung von mir:

«Inwiefern das jetzt aber jeweils Objektivationen oder Vergegenständlichung von den jeweils, von dem jeweiligen Denken der Person an für sich sind oder welche Facetten davon in der Gruppendiskussion noch ausgehen, dass muss man, glaube ich schon (ehm) irgendwie klar haben, dass das nur Facetten hauptsächlich sind und nicht eben die Vorstellung von einer Person. Also es ist vielleicht ein Gruppenergebnis als Konsens, worauf sich die Leute einlassen konnten, gleichwohl könnte man jetzt aus dieser Erfahrung oder seiner Formulierung deuten, dass es eben längst nicht bei allen dazu führt, dass alle eben einen Konsens damit verbinden, sondern auch das eine Form von Aufgabenbewältigung sein kann» (Sprachmemo 003, 1:04-1:55 min, 17.04.2015).

Zum Schreiben der Wikibeiträge sowie zur Kompensation des Blocktermins erfolgte in den ersten drei Wochen keine Präsenzsitzung. Den Studierenden wurde stattdessen die Möglichkeit eröffnet, die regulären Zeiten für Gruppentreffen in der ComputerStudienWerkstatt zu nutzen oder sich selbst zu organisieren. Zudem konnten Studierende Beratungsangebote in Form von Sprechstunden annehmen sowie Rückmeldungen zu ihren Textprodukten anfragen. Studierenden, die im Rahmen des Blocktermins nicht anwesend waren, wurde ebenfalls die Möglichkeit eingeräumt, die zu erbringenden Leistungen bis zum 30. April 2015 abzugeben. Diese Möglichkeit nutzten die Studierenden in selbstorganisierten Gruppen bzw. teilweise in Einzelarbeit. In unterschiedlichem Umfang nahmen Studierende dabei Beratungsangebote in Form von Sprechstunden wahr.

Erwähnenswert ist in diesem Zusammenhang die Erfahrung eine Beratungssituation in einer Sprechstunde. Im Gespräch mit einer Person, der studentischen Tutorin und mir, zeigten verschiedene Schwierigkeiten in der praktischen Beratung von Studierenden sowie interessante sprachliche Phänomene zum Ausdruck mit Metaphern. Die Person formulierte den expliziten Vergleich «Bildung ist wie die niederprasselnde Sonne auf die Erde». Beim Versuch diese Metaphorik nachvollziehen zu können, stellten wir der Person verschiedene Frage zu einzelnen Elementen des Vergleichs. Die Erläuterungen erfolgten dabei in Form weiterer, weitgehend routinisierter Metaphern ${ }^{189}$. Der Wechsel zwischen unterschiedlichen Metaphern erschien mir aus einer

189 Zum Beispiel verwies die Person darauf, dass es bei Bildung jeweils einen Start- und einen Zielpunkt gibt. Im Verlauf erfolgte allerdings kein Bezug zur Sonne und zur Erde. Stattdessen wurde im Gespräch das Bild der Geburt als Startpunkt benannt (Sprachmemo 004, 1:10-1:25 min, 28.04.2015). 
forschenden Perspektive einerseits interessant. Im Kontext der Aufgabenstellung, eine Ad-hoc-Metapher bzw. eine Gruppenmetapher zu entwickeln und deren Grenzen zu diskutieren, erlebte ich die Beratungssituation in meiner Rolle als Lehrenden andererseits als Herausforderung. In einem Sprachmemo habe ich diese Erfahrung wie folgt beschrieben:

«[...] und das ist ja interessant, weil [die Person] an der Stelle verschiedene metaphorische Ausdrücke verwendet hat, um uns bestimmte Dinge zu erklären, die aus [ihrer] Perspektive zur Bildung gehören. Aber es war eben keine konsistente Metapher, sondern es wurden immer wieder andere Bilder verwendet [...], aber es war eben irgendwie interessant, weil dieser Bildungsbegriff eben so facettenreich war und gleichzeitig so unpräzise und (kurze Pause) es auch schwierig war [die Person bzw. das Verständnis der Person] sichtbar zu kriegen. Genau, das mal als Memo: Diese Metaphern bringen auch Schwierigkeiten mit sich, weil sich auch bestimmte Sachen eben nicht als (ehm) erfahrbar [erweisen]. Als Bildbereiche der Metaphern hat [die Person] nicht einen erfahrbaren Bereich [verwendet] sondern eben andere Konstrukte wie «Endlosschleife» oder sowas abstraktes wie Sonne und Erde wo wir nur ein Modell dazu haben» (Sprachmemo 004, 1:04-3:12 min, 28.04.2015).

Das Sprachmemo kann als Indikator dafür gewertet werden, dass es mir in der Rolle als Lehrender zu diesem Zeitpunkt wichtig war, dass Studierende versuchen eine möglichst konsistente Metaphorik zu formulieren bzw. zu entwickeln. Der Wechsel zwischen zahlreichen - weitgehend routinisierten - Metaphern war für mich als Lehrender irritierend. Ich hatte vermutet, dass das Erkennen von Bildbrüchen die Chance bietet, die Grenzen bestimmter Bilder und Ausdrucksweisen zu identifizieren. Dieser Vermutung war aber die implizite Annahme vorausgegangen, dass das Verwenden unterschiedlicher Bilder auch als Bruch wahrgenommen wird. In der Beratungssituation erlebten nur die studentische Tutorin und ich die Äusserungen als widersprüchlich. Daher lohnte es sich, in der Auswertung (1.) die Darstellungsformen der Ad-hoc-Metaphern zu betrachten (Kap. 7.4.3.1) und (2.) die Verwendung routinisierter Metaphern zu berücksichtigen (Kap. 7.4.3.2). Dies erfolgte, wie von Marsch und Krüger (2007) vorgeschlagen, durch die Beachtung impliziter und expliziter metaphorischer Formulierungen (Kap. 3.4.3).

\subsubsection{Materialien und Interaktionen über Moodle}

Die Studierenden erhielten Orientierungsmaterialien zur ersten Phase im MoodleKurs in Form von organisatorischen Informationen zum Projekt der Lehrveranstaltung sowie zur Dokumentation der geblockten Präsenzveranstaltung. Allgemeine Informationen und Ankündigungen zur Veranstaltung wurden über ein Nachrichtenforum 
des Moodle-Kurses verschickt. Die Dokumentation der Arbeitsprodukte und Zwischenergebnisse der Präsenzveranstaltungen erfolgte in Form von Moodle-Foren, welche für die jeweiligen Arbeitsgruppen angelegt wurden. Über einzelne Threads wurden Bilder der Flipcharts sowie der erstellten Videobotschaften von der studentischen Mitarbeiterin und mir hochgeladen (Tab. 7.3). Zudem sollten die Foren den Studierenden eine Möglichkeit bieten, die im Blocktermin begonnen Arbeiten in einem geschützten Rahmen fortführen zu können (aber nicht zu müssen). Die Möglichkeit über die angelegten Moodle-Foren zusammenzuarbeiten, wurde nur von einer studentischen Arbeitsgruppe aktiv genutzt (Tab. 7.3).

\begin{tabular}{|l|l|l|l|l|}
\hline & \multicolumn{2}{l}{ Lehrende } & \multicolumn{2}{l|}{ Studierende } \\
\cline { 2 - 5 } & Threads & Antworten & Threads & Antworten \\
\hline Didaktik AG 1 & 2 & 0 & 1 & 0 \\
\hline Didaktik AG 2 & 2 & 1 & 1 & 0 \\
\hline Didaktik AG 3 & 2 & 1 & 1 & 11 \\
\hline Didaktik AG 4 & 2 & 0 & 1 & 0 \\
\hline Didaktik AG 5 & 0 & 0 & 3 & 0 \\
\hline Didaktik AG 6 & 1 & 0 & 1 & 0 \\
\hline Didaktik AG 7 & 2 & 1 & 0 & 0 \\
\hline Bildung AG 1 & 3 & 0 & 0 & 2 \\
\hline Bildung AG 2 & 2 & 1 & 1 & 2 \\
\hline Bildung AG 3 & 2 & 0 & 1 & 0 \\
\hline Bildung AG 4 & 1 & 0 & 0 & 2 \\
\hline
\end{tabular}

Tab. 7.3: Aktivitätsübersicht der Moodle-Foren der ersten Seminarphase in Form erstellter Threads und Antworten im Auswertungszeitraum vom 1.04.2015 bis zum 03.05.2015

Durch das Angebot einer Rückmeldung sollten die Studierenden zum Nachdenken und Weiterdenken angeregt werden. Zum anderen dienten die Rückmeldungen der Qualitätssicherung des Buchprojektes. Die Rückmeldungen erfolgten in Form von Antworten bzw. Threads in den Arbeitsgruppen-Foren der Studierenden. Die Feedbackbeiträge waren nach folgendem Schema aufgebaut: (1.) Einschätzung des Wikibeitrages, (2.) inhaltliches Nachfragen bzw. Reflexionsfragen, (3.) formale Hinweise zur Überarbeitung. Für die Einschätzung der Wikibeiträge wurde zwischen drei Niveaustufen unterschieden, die Einstufung wurde im kommunikativen Austausch der studentischen Mitarbeiterin getroffen ${ }^{190}$. Die inhaltlichen Nachfragen bzw. Reflexionsfragen sowie die formalen Hinweise zur Überarbeitung wurden für die verschiedenen Beiträge jeweils individuell formuliert. Aufgrund der begrenzten Zeit als Lehrender wurden die Rückmeldungen jeweils auf maximal drei zentrale inhaltliche

190 Zur Rückmeldung der jeweiligen Einschätzung der studentischen Beiträge wurde zwischen drei Formulierungen unterschieden (Anhang D.1.2.2). 
Aspekte beschränkt ${ }^{191}$. Eine exemplarische Rückmeldung zu einem Wikibeitrag ist im Anhang dokumentiert (Anhang D.1.2.3).

\subsubsection{Erleben und Thematisierung von Interaktionen mit der Wikicommunity}

Zum Ende der ersten Abgabefrist erstellten die Studierenden ihre Beiträge im Wikibook. Dabei zeigte sich, dass das Anlegen einer neuen Wikiseite mithilfe der Wikisyntax für viele Studierende eine Herausforderung darstellte. Zur Erstellung einer Wikiseite im Rahmen des Wikibooks mussten die Studierenden die Namenskonvention bzw. den Pfad beachten. Dazu gehörten die Angabe des Wikibooks «Lehren, Lernen und Bildung metaphorisch verstehen», des Kapitelnamens "Vorstellung» sowie die Angabe des Namens der Wikiseite. Für die Metapher «Lehren und Lernen ist wie Wandern» sah die zugehörige Wikisyntax zur Erstellung einer neuen Wikiseite beispielsweise wie folgt aus:

\#\#\# [[Lehren, Lernen und Bildung metaphorisch verstehen/ Vorstellungen/ Lehren und Lernen ist wie Wandern|Lehren und Lernen ist wie Wandern]]

Neben der Angabe des Pfades in der richtigen Reihenfolge war die Angabe der Zeichen «\#», «[» und «]» notwendig, damit ein Link auf eine neue Wikiseite in Form einer nummerierten Liste durch den Wiki-Editor erstellt wird. Die Erstellung entsprechender Einträge im Wikibook erfolgte durch die Studierenden in unterschiedlicher Weise. Eine studentische Arbeitsgruppe mit Vorerfahrungen im Umgang mit Wikis konnte angemessene Links erstellen. Weitere studentische Arbeitsgruppen orientierten sich an der entsprechenden Syntax. Dies gelang jedoch den drei folgenden studentischen Arbeitsgruppen nicht in angemessener Weise, wie sich am folgenden Versionsverlauf der Startseite ${ }^{192}$ exemplarisch zeigen lässt:

- Version vom 29. April 2015, 13:06 Uhr: Bearbeitung der Zeile 25 durch eine unangemeldete Person zu «\#\#\#[[Lehren und Lernen ist wie Wandern]]» (+ 41 Bytes) ohne Bearbeitungszusammenfassung

- Version vom 29. April 2015, 13:18 Uhr: Bearbeitung der Zeile 25 durch eine unangemeldete Person zu «\#\#\#[[Lehren, Lernen und Bildung metaphorisch verstehen/ Vorstellungen\#Lehren und Lernen ist wie Wandern|Lehren und Lernen ist wie Wandern]]» (+ 99 Bytes) ohne Bearbeitungszusammenfassung

191 Die Rückmeldungen zu den Metaphern wurden zu zwei Zeitpunkten formuliert. Studentische Arbeitsgruppen, die ihre Texte bereits vor der Abgabefrist zur Verfügung gestellt hatten, erhielten eine Rückmeldung am 27. April 2015, d. h. etwa eine Woche bevor die vorläufige Endfassung im Rahmen eines Wikibeitrages formuliert sein musste. Studentische Beiträge, die nach dem 27. April 2015 über ein Arbeitsgruppenforum bzw. über Wikibooks formuliert worden waren, erhielten in der zweiten Seminarphase eine schriftliche Rückmeldung.

192 Online verfügbar unter https://de.wikibooks.org/wiki/Lehren,_Lernen_und_Bildung_metaphorisch_ verstehen (01.01.2019). 
- Version vom 29. April 2015, 13:26 Uhr: Bearbeitung der Zeile 25 durch eine unangemeldete Person zu «\#\#\#[[Lehren und Lernen ist wie Wandern|Lehren und Lernen ist wie Wandern]]» (- 65 Bytes) ohne Bearbeitungszusammenfassung

- Version vom 29. April 2015, 14:35 Uhr: Bearbeitung der Zeile 25 durch den angemeldeten Nutzer Juetho zu «\#\#\#[[Lehren, Lernen und Bildung metaphorisch verstehen/ Vorstellungen/ Lehren und Lernen ist wie Wandern|Lehren und Lernen ist wie Wandern]]» (+ 66 Bytes) mit der Bearbeitungszusammenfassung «Wandern» als Kapitel ins Buch gesetzt»

Die vier Bearbeitungen erfolgten innerhalb von 89 Minuten durch mindestens zwei verschiedene Personen. Eine unangemeldete Person fügte zunächst die dargestellten Zeichenfolgen hinzu. Durch die Verwendung des Symbols Raute «\#» statt Slash «/» funktionierte die eingegebene Zeichenfolge in der zweiten Bearbeitung nicht zur Erstellung einer neuen Wikiseite. In der dritten Bearbeitung wurden verschiedene Textelemente entfernt, wodurch die in der ersten Bearbeitung erstellte Seite wieder zugänglich war, jedoch der Wikibeitrag nicht im zugehörigen Wikibook verortet wurde. Dieser Fehler wurde vom Wikibookianer Juetho ${ }^{193}$ in der vierten Bearbeitung korrigiert. Juetho unterstützte in den folgenden Tagen zwei weitere studentische Arbeitsgruppen bei Problemen mit der Erstellung einer Wikiseite. Ferner wurde von Juetho eine Arbeitsanleitung zur Erstellung von Wikiseiten am 1. Mai 2015 erstellt (Abb. 7.4). In einer Schritt-für-Schritt-Anleitung wurde Studierenden erklärt, wie eine Wikiseite für das Wikibook erstellt werden kann.

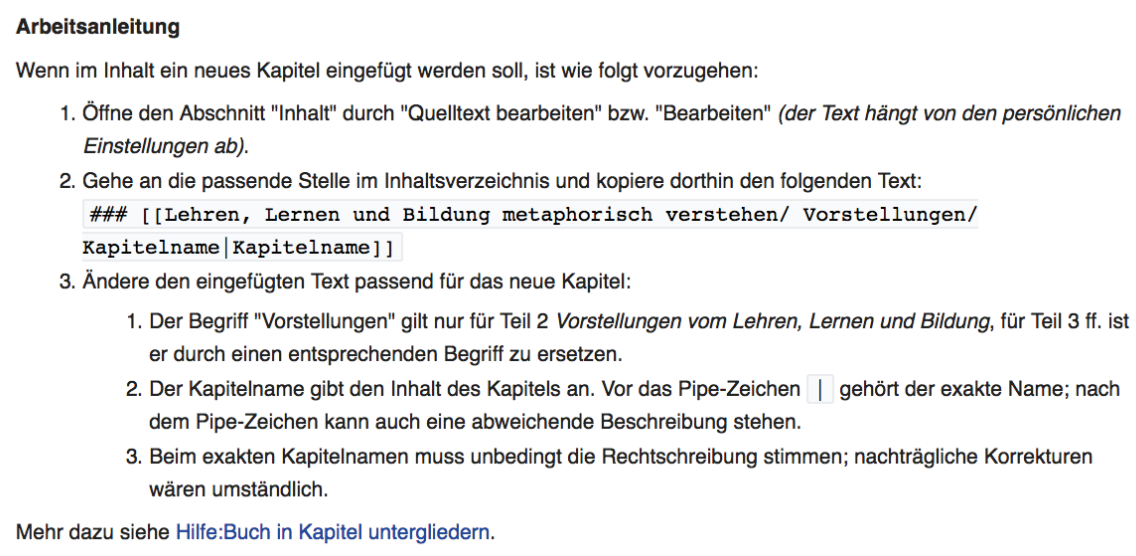

Abb. 7.4: Screenshot der Arbeitsanleitung des Wikibooks auf der Startseite (online abgerufen am 1. Mai 2015).

193 Juetho ist der öffentliche Benutzername eines Wikibookianers, der sich u. a. in seiner Rolle als Wikibook-Administrator aktiv an der Mitgestaltung des Wikibookprojektes eingebracht hat. Das Benutzerprofil ist online zugänglich: https://de.wikibooks.org/wiki/Benutzer:Juetho (01.01.2019). 
Neben den Beiträgen auf der Startseite formulierten Mitglieder der Wikicommunity auch Kommentare zum Wikibookprojekt. Diese erfolgten auf der Diskussionsseite des Wikibooks sowie in Form eines Beitrages auf der Diskussionsseite meines Wikibookprofils. Im Fokus der Kommentare standen «Allgemeine Anmerkungen» zur Formatierung, zur Arbeit mit der Wikistruktur sowie zur Strukturierung der einzelnen Seiten zur Darstellung der Metaphern (Abb. 7.5). Im Zeitraum vom 18. April 2015 bis zum 29. April wurde die Diskussionsseite elf Mal bearbeitet. Die Bearbeiter waren die angemeldeten Wikibookianer Juetho (7 Bearbeitungen) sowie Stephan Kulla ${ }^{194}$ (1 Bearbeitung) und ich in Form von Reaktionen auf die Anmerkungen (2 Bearbeitungen).

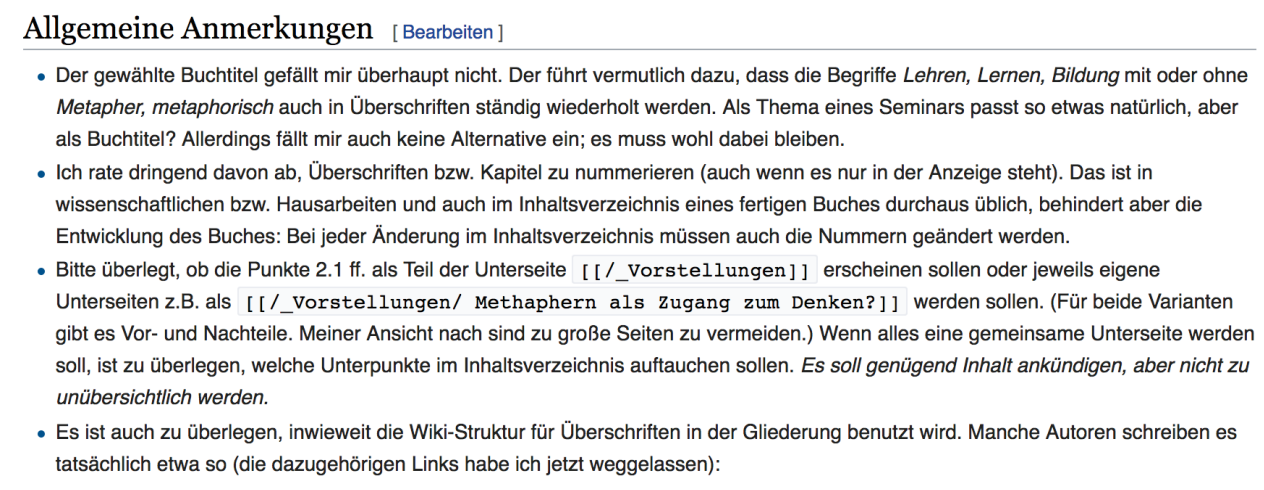

Abb. 7.5: Screenshot der Diskussionsseite der Startseite des Wikibooks (Anhang E.7).

Diese Kommentare waren für mich als Lehrender aus verschiedenen Gründen erfreulich. Zum einen kann durch die formulierten Kommentare gezeigt werden, dass das mit Studierenden bearbeitete Wikibook auch von Mitgliedern der Wikicommunity bzw. Menschen ausserhalb des Seminars zur Kenntnis genommen wurde. Die Kommentare wurden als Indikator dafür gedeutet, dass die erstellten Texte der Studierenden gelesen werden. Zudem ermöglichten die Kommentare ein Blick von «Aussen» auf das Wikibookprojekt sowie eine Unterstützung im Umgang mit der Wikisyntax. Die Kommentare beschränkten sich nicht nur auf die Diskussionsseite, vielmehr wurden verschiedene Bearbeitungen bzw. Überarbeitungen zur Formatierung auf den Inhaltsseiten der Studierenden durchgeführt. Diese Bearbeitungen, die durch die Versionsgeschichte sichtbar werden, wurden als geeignete Indikatoren betrachtet, um die von Iske und Marotzki (2010) markierten Prinzipien von Wikis in den Präsenzveranstaltungen thematisieren zu können. In einem Sprachmemo habe ich meine Erfahrungen als Lehrender in der Interaktion mit Mitgliedern der Wikicommunity wie folgt beschrieben:

194 Stephan Kulla ist der öffentliche Benutzername eines Wikibookianers, der sich $u$. a. in seiner Rolle als Wikibook-Administrator aktiv an der Mitgestaltung des Wikibookprojektes eingebracht hat. Das Benutzerprofil ist online zugänglich: https://de.wikibooks.org/wiki/Benutzer:Stephan_Kulla (01.01.2019). 
«Also im Moment könnte ich den Herrn ein Stück weit knutschen, weil er eben diese Interaktionen provoziert oder zumindest sichtbar macht, dass Menschen von ausserhalb sich für diese Produkte interessieren und Interaktionen entstehen. [...] Also, dass es tatsächlich gelesen wird und nicht im Sinne von: wir schreiben das jetzt irgendwo online und niemand sieht das eigentlich, sondern es wird sogar mehr oder weniger von Schritt zu Schritt verfolgt und kommentiert, was wir da eben tun und was wir da lassen» (Sprachmemo 005, 2:15-3:30 min, 29.04.2015).

Für die Planung der nächsten Veranstaltungen waren diese Erfahrungen aus zwei Gründen relevant. Zum einen eröffnete sich die Möglichkeit, an den konkreten Interaktionen exemplarisch zu thematisieren, in welcher Weise Artikel und Beiträge in Wiki-Communities ausgehandelt werden. So wurde es möglich, Lernhandlungen zum Durchschauen und Beurteilen von Bedingungen der Wissensproduktion anzuregen und zu unterstützen (Kap. 6.4.2.2). Zum anderen wurde die Notwendigkeit, den Studierenden mehr Unterstützung bei der Erstellung ihrer Beiträge auf Wikibooks bereitzustellen, ersichtlich. Für die Analyse des Projektes war es zudem interessant, systematisch den Entstehungsprozess der verschiedenen Wikiseiten zu untersuchen. Die forschungsmethodische Umsetzung dieser Analyse erfolgte in Form einer statistischen Auswertung der verschiedenen Daten der Versionsgeschichte (Kap. 3.4.2.2, 7.3.4).

\subsubsection{Phase II: Erarbeitung erziehungswissenschaftlicher «Denkwerkzeuge»}

In Anknüpfung an die Darstellung der ersten Seminarphase werden erneut drei Schwerpunkte gewählt: (1.) Gestaltung und Wahrnehmung der Präsenzveranstaltungen, (2.) Erfahrungen mit Moodle, Planungskorrektur und Partizipation sowie (3.) Interaktionen mit dem Wikibook und Mitgliedern der Wikicommunity.

\subsubsection{Gestaltung und Wahrnehmung der Präsenzveranstaltungen}

Die erste Präsenzveranstaltung in der zweiten Seminarphase wurde zu einer reflexiven Auseinandersetzung mit den bisherigen Wiki-Erfahrungen genutzt. Die weiteren Präsenztermine dienten der Diskussion erziehungswissenschaftlicher "Denkwerkzeuge» auf Basis des jeweiligen Bearbeitungsstandes der studentischen Arbeitsgruppen. Der Rückblick auf die bisherigen Erfahrungen erfolgte in drei Schritten: (1.) Betrachtung der Reaktionen der Wikibook-Community aus medienbildungstheoretischer Perspektive, (2.) Unterscheidung unterschiedlicher Wissensformen und Einschätzung der bisherigen Texte und (3.) Planung des weiteren Vorgehens und der Gruppenwahl. Im ersten Schritt sollten die beschriebenen Interaktionen mit den Mitgliedern der 
Wikicommunity genutzt werden, um Studierende für die unterschiedlichen Prinzipien von Wikis in Anlehnung an Iske und Marotzki (2010) zu sensibilisieren. Dafür wurden die drei Prinzipien «Reflexivität», «Partizipation» sowie «Prozessualität» von Iske und Marotzki (2010) am Beispiel des Wikibooks erläutert. Das Prinzip der Reflexivität diskutieren Iske und Marotzki (2010) z. B. hinsichtlich der Artikulation eigener Erfahrungen in Form von Wikibeiträgen, welche zum Ausgangspunkt für eine weitere individuelle und kollaborative Nutzung werden kann. Dies zeigt sich im Rahmen des Wikibookprojektes u. a. in Form von Kommentaren auf der Diskussionsseite. Ausgehend von den Wikibeiträgen der Studierenden formulierte der Wikibookianer Juetho verschiedene Hinweise zur Gestaltung der Wikiseiten (Abb. 7.6). Im zweiten Schritt erfolgte die bereits geplante Diskussion der im Rahmen des Seminars und des Wikibooks thematisierten Wissensformen (Kap. 6.6.1.2). Bei der Diskussion der unterschiedlichen Wissensformen konnten Studierende nachvollziehen, dass das Wikibook bisher vor allem Alltagswissen bzw. Alltagswissen in verdichteten Metaphern beinhaltet (Terhart 2009). In Anlehnung an das Leitbild von Wikibooks.org benötigt ein Wikibook jedoch "gesichertes Wissen» (Wikibooks 2016), z. B. in Form von wissenschaftlichen Modellen. Diese Anforderung an ein Wikibook wurde als ein Argument verwendet, um eine Auseinandersetzung mit erziehungswissenschaftlichen Modellen zu begründen. Dieses Argument war für viele Studierende nachvollziehbar.
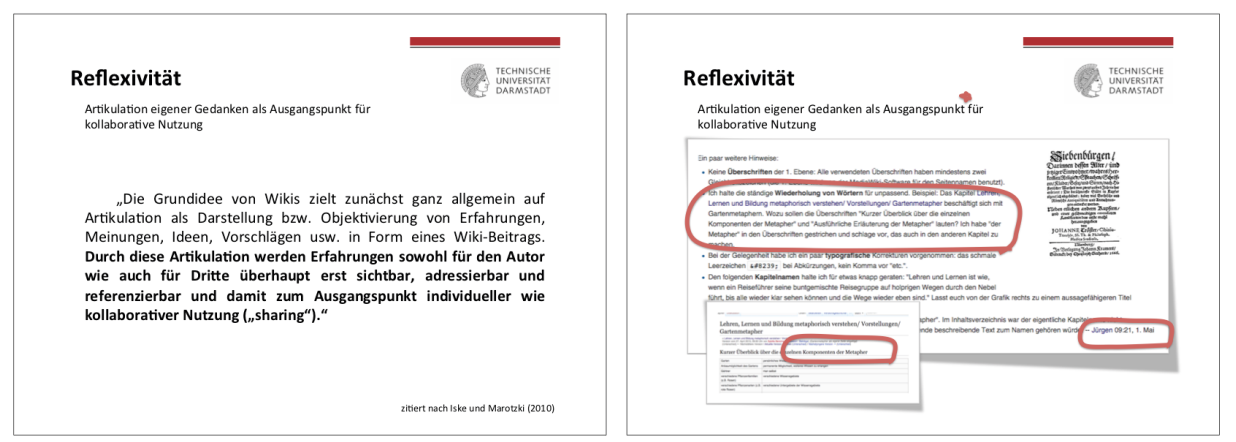

Abb. 7.6: Folien zur Erläuterung des Prinzips der Reflexivität am Beispiel von Kommentaren (eigene Darstellung).

Das weitere Vorgehen zur Erarbeitung erziehungswissenschaftlicher Denkwerkzeuge sowie zur Gestaltung des Wikibooks beinhaltete zwei Aspekte. Es wurden die konkreten Kommentare zum Wikibook diskutiert und zudem besprochen, wie mit den Kommentaren und Anregungen durch die Mitglieder der Wikicommunity umgegangen werden soll. Dabei entwickelte sich eine lebendige Diskussion über die verschiedenen Kommentare. Die Studierenden und Lehrenden einigten sich u. a. darauf, dass die Beitragsstruktur vereinheitlicht wird und die Darstellung der Metaphern durch tabellarische Übersichten zu ergänzen sind (Anhang E.3). Um Studierenden Partizipationsmöglichkeiten zu eröffnen, wurden ihnen für die zweite Seminarphase 
verschiedene erziehungswissenschaftliche Denkwerkzeuge zur Auswahl gestellt (Kap. 6.6.3). Zudem hatten die Studierenden die Möglichkeit, eigene Vorschläge einzubringen. Es wurden jedoch keine Modelle von Studierenden vorgeschlagen. Nach den Präsenzveranstaltungen beschrieb ich meinen Eindruck: «die Gruppen haben sich auf die entsprechenden Begriffe festgelegt, vermutlich ohne genau zu wissen, worauf sie sich da einlassen» (Sprachmemo 010, 2:40-2:50 min, 04.05.2015) ${ }^{195}$.

Die folgenden drei Präsenzveranstaltungen im Rahmen der zwei Seminare begannen jeweils mit einer zehn bis fünfzehnminütigen Diskussions- und Fragerunde zur gemeinsamen Gestaltung des Wikibooks (u. a. zur Darstellung erziehungswissenschaftlicher Denkwerkzeuge, zur Gestaltung des Inhaltsverzeichnisses, zur Verwendung einer geschlechtergerechten Sprache). Im Anschluss hatten die studentischen Arbeitsgruppen die Aufgabe, ihre jeweils ausgewählten didaktischen Modelle bzw. Lerntheorien zur Diskussion zu stellen. Aufgrund der unterschiedlichen Anzahl der Teilnehmerinnen und Teilnehmer der Seminargruppen (Kap.7.2.1) variierte die dafür zur Verfügung stehende Zeit ${ }^{196}$. Die studentischen Arbeitsgruppen nutzten dabei unterschiedliche Varianten zur Darstellung ihrer bis dahin erfolgten Auseinandersetzung mit didaktischen Modellen (z. B. eigene Textentwürfe zum Lesen, Kurzvorträge zur Aufarbeitung einzelner Facetten eines Modells, das gemeinsame Lesen von Sekundärliteratur). Zudem zeigten sich unterschiedliche Formen der Zusammenarbeit im Rahmen der studentischen Arbeitsgruppen.

Zur Gestaltung der Wikibeiträge wurde im Rahmen der Präsenztermine die Frage diskutiert, inwiefern die Einbindung von schematischen Darstellungen und Abbildungen möglich ist. Insbesondere zur Darstellung der analytischen Dimension didaktischer Modelle hielten es mehrere Studierende für sinnvoll, konkrete Darstellungen zu verwenden. Beim Versuch, Grafiken und Abbildungen in einen Wikibeitrag zu integrieren, wurden die Studierenden aufgefordert, eigene Darstellungen hochzuladen und der Lizenzierung unter einer Creative Commons Lizenz zuzustimmen. Diese Erfahrung sorgte bei verschiedenen Studierenden für Irritationen, weshalb die Frage im Rahmen der Präsenzsitzungen zu thematisiert wurde. Zum Umgang mit dieser Herausforderung erfolgte $u$. a. eine Anfrage an einen Verlag, ob die eigene Darstellung eines Schaubildes für einen Wikibeitrag unter einer Creative Commons Lizenz veröffentlicht werden durfte. Weitere studentische Arbeitsgruppen recherchierten relevante Darstellungen unter offenen Lizenzen.

195 Bei der Präsenzsitzung waren nicht alle Teilnehmerinnen und Teilnehmer der Seminare anwesend. Entsprechend konnten einige Studierende weder die Diskussion über das weitere Vorgehen mitgestalten, noch waren sie in die Gruppenauswahl involviert. Zum Umgang mit dieser Problematik erfolgte eine Rund-E-Mail mit den relevanten Informationen verschickt (Anhang D.1.3.1).

196 Im Seminar «Lehren und Lernen metaphorisch verstehen?» musste aufgrund von drei Themenpräsentation an einem Tag die Vorstellungszeit der studentischen Arbeitsgruppen auf 25 Minuten reduziert werde. Im Seminar «Bildung metaphorisch verstehen?» blieb hingegen deutlich mehr Zeit für die einzelnen Arbeitsgruppen, so dass auch weiterführende Themen im Rahmen einer Seminarsitzung diskutiert werden konnten (z. B. zur Frage, warum sich Lehramtsstudierende mit erziehungswissenschaftlichen Modellen und Theorien auseinandersetzen müssen). 


\subsubsection{Erfahrungen mit Moodle, Planungskorrektur und Partizipation}

Mit dem Einsatz von Moodle wurden in der zweiten Projektphase die folgenden Ziele verfolgt: (1.) Dokumentation der Präsenzveranstaltungen für die Studierenden, (2.) Bereitstellung von Orientierungsangeboten, (3.) Eröffnung von Austausch- und Partizipationsmöglichkeiten sowie (4.) Rückmeldungen zu den Textentwürfen der Studierenden. Die Dokumentation der Präsenzveranstaltungen erfolgte in Form von Materialien zur Organisation und zum Ablauf der Veranstaltung. Orientierungsangebote wurden den Studierenden zum einen zur formalen Gestaltung wissenschaftlicher Texte gegeben und zum anderen wurden Literaturempfehlungen zur Auseinandersetzung mit den gewählten Themen veröffentlicht. Zur Orientierung der Studierenden wurde zudem der Sinn der zweiten Projektphase in einem Textfenster im Moodlekurs formuliert ${ }^{197}$.

Die Ankündigungen sowie die Benachrichtigungen aller Teilnehmerinnen und Teilnehmer erfolgten hinsichtlich allgemeiner Projektinformationen über das Nachrichtenforum ${ }^{198}$. Die bereitgestellten Materialien zur Organisation der Veranstaltung umfassten den Foliensatz zur Projektplanung sowie die im Seminar vorgenommene Gruppen- und Terminzuordnung. Als Orientierungshilfe zum wissenschaftlichen Schreiben wurden den Studierenden zwei Kapitel ${ }^{199}$ von Kruse (2007) sowie die bereits thematisierte Formatvorlage über Moodle zur Verfügung gestellt (Anhang D.20). Die Formatvorlage wurde auch vor dem Hintergrund erstellt, dass die Artikel in der ersten Seminarphase sehr unterschiedlich strukturiert waren. Sie sollte zur Vereinheitlichung der Beiträge dienen und eine Orientierung dafür geben, was von den Studierenden erwartet wird. Zur inhaltlichen Orientierung erhielten die Studierenden jeweils themenspezifische Literaturempfehlungen. Die Vergabe erfolgte in den jeweiligen Arbeitsgruppenforen der zweiten Projektphase ${ }^{200}$.

Die Foren der Arbeitsgruppen sollten den Studierenden zudem Austauschmöglichkeiten untereinander bereitstellen. Die Studierenden nutzten die Foren überwiegend zur Bereitstellung ihrer Textentwürfe um Rückmeldungen zu erhalten. Die Ausnahme bildete jeweils eine Arbeitsgruppe, welche ihre gemeinsame Texterstellung über das zur Verfügung gestellte Moodle-Forum organisierte (Tab. 7.4). Die Anzahl der erstellten Threads und Antworten bieten Indikatoren, um die verschiedenen Umgangsweisen zu verdeutlichen.

197 Die Formulierung lautete: «Der Sinn der zweiten Phase ist es, eine Auseinandersetzung mit didaktischen sowie lern- und bildungstheoretischen Überlegungen anzuregen. Durch die Diskussion und Verschriftlichung eurer Gedanken wird sichtbar, welche Aspekte ihr als relevant erachtet und welche Aspekte für euch ggf. weniger von Bedeutung sind. So werden eure Gedanken einerseits für Feedback zugänglich. Andererseits bietet sich die Möglichkeit, eure Vorstellungen über Lehren und Lernen (welche mithilfe von Metaphern bereits versprachlicht wurden) zu hinterfragen».

198 Eine exemplarische Nachricht war die E-Mail zum «Beginn der zweiten Projektphase im Wikibookprojekt» mit Informationen, die im Rahmen der ersten Präsenzsitzung nicht thematisiert werden konnten (Anhang D.1.3.1).

199 Die Kapitel waren «Einen Diskurs konstruieren» (Kruse 2007, 68 ff.) und «Textverständlichkeit und wissenschaftlichem Schreiben» (ebd., 92 ff.).

200 Eine exemplarische Literaturempfehlung ist im Anhang D.1.3.2 dokumentiert. 


\begin{tabular}{|l|l|l|l|l|}
\hline & \multicolumn{2}{|l|}{ Lehrende } & \multicolumn{2}{l|}{ Studierende } \\
\cline { 2 - 5 } & Threads & Antworten & Threads & Antworten \\
\hline Didaktik AG 1 & 1 & 2 & 2 & 11 \\
\hline Didaktik AG 2 & 2 & 0 & 0 & 0 \\
\hline Didaktik AG 3 & 1 & 1 & 1 & 0 \\
\hline Didaktik AG 4 & 1 & 1 & 1 & 3 \\
\hline Didaktik AG 5 & 1 & 1 & 1 & 1 \\
\hline Didaktik AG 6 & 1 & 0 & 1 & 0 \\
\hline Didaktik AG 7 & 1 & 1 & 1 & 0 \\
\hline Bildung AG 1 & 1 & 2 & 1 & 1 \\
\hline Bildung AG 2 & 0 & 1 & 4 & 12 \\
\hline Bildung AG 3 & 1 & 1 & 1 & 0 \\
\hline Bildung AG 4 & 1 & 1 & 4 & 0 \\
\hline
\end{tabular}

Tab. 7.4: Aktivitätsübersicht der Moodle-Foren der zweiten Seminarphase in Form erstellter Threads und Antworten im Auswertungszeitraum vom 4.05.2015 bis zum 23.06.2015.

Nachdem die Studierenden ihre Textentwürfe über Moodle zur Diskussion stellten, nutzten die Lehrenden die Foren zum Posten von Rückmeldungen. Die Zielstellung der Rückmeldungen umfasste analog zur ersten Projektphase (Kap. 7.2.2) zwei Ebenen: Rückmeldung zur inhaltlichen Auseinandersetzung mit dem gewählten Thema sowie zur Qualitätssicherung der Texte im Wikibook. Der erste Eindruck der formulierten Texte für das Wikibook hat mich in meiner Rolle als Lehrende überrascht. Diese Überraschung ist im folgenden Sprachmemo dokumentiert:

«Ich lese mir gerade die ersten Formulierungen für die erziehungswissenschaftlichen Denkwerkzeuge durch und salopp formuliert, denke ich: «Oh

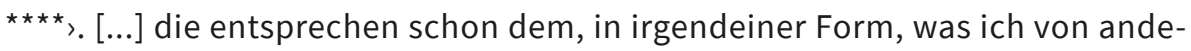
ren Seminaren kenne, z. B. aus dem Seminar «Föhn mich nicht zu! - Didaktische Fallanalysen, in der Art und Weise wie die Texte geschrieben sind. Aber lustiger Weise denke ich dieses Mal: «Oh $\mathrm{F}^{\star \star} k$ ». Auch mit dem Hinblick auf: Hey das ist ein Wikibookprojekt der TU Darmstadt und von mir auch ein Stück weit und irgendwie sollte das auch vernünftig formuliert sein und (kurze Pause) da gibt es bei den jeweiligen Texten aber glaube ich noch einiges zu tun. Die Frage, die ich mir gerade stelle ist: Wie kann ich Feedback formulieren, sodass sie sich da noch einmal ransetzen. Weil, dass [die Kritik] beginnt sozusagen bei, wie konstruiere ich einen Diskurs, wie mache ich deutlich welche Aussagen von mir sind und welche nicht. Also dann haben sie an der Strukturierung ein Stück weit Dinge verändert, also entgegen der Formatvorlage gearbeitet. Mitunter sind die Zuordnungen nicht ganz passend. [...] Und das beginnt schon damit, das mitunter auch Absätze nicht so verwendet worden sind, dass tatsächlich Sinneinheiten daraus entstehen, was mich wiederum irritiert. Genau 
das sozusagen als erstes, also die Frage, wie kriegt man jetzt eigentlich Beratungsangebote oder Angebote geschaffen, um die Qualität der Texte zu steigern» (Sprachmemo 015, 0:05-1:55 min, 16.05.2015).

Um den Studierenden hilfreiche und vergleichbare Rückmeldungen bereitzustellen, wurde im Verlauf der zweiten Seminarphase zum einen entschieden, für alle Textentwürfe jeweils einen Feedbackbogen zu verwenden. Zum anderen wurden die jeweiligen Textentwürfe annotiert, um die Einschätzungen des Feedbackbogens zu plausibilisieren und Hinweise zu konkreten Formulierungen geben zu können. Der Feedbackbogen basierte auf den Materialien des Arbeitsbereiches «Allgemeine Pädagogik mit dem Schwerpunkt Medienpädagogik» und wurde hinsichtlich des Umfangs der Kriterien für die zu schreibenden Beiträge reduziert und angepasst. Es wurde zwischen formalen sowie inhaltlichen Kriterien unterschieden. Die formalen Kriterien umfassten (F1.) korrekte Belege und Zitate, (F2.) korrektes Literaturverzeichnis, (F3.) Berücksichtigung der Formatvorlage zur Strukturierung des Textes sowie (F4.) Aufgreifen der behandelten Literatur sowie das Heranziehen von hinreichend weiterer Literatur. Die Kriterien zur Einschätzung der inhaltlichen Qualität des Textes sowie der Auseinandersetzung der Studierenden lauteten:

- Relevantes Fachwissen (Fachliteratur) ausgewählt und projektbezogene Sachverhalte sachlich richtig und präzise dargestellt

- Struktur \& roter Faden: Stringenz in der Darstellung relevanter Aspekte, sinnvolle Gliederung mithilfe der Formatvorlage

- Entwicklung von Argumenten in Auseinandersetzung mit Positionen anderer (Fachdiskurs)

- Quellentransparenz: Differenz zwischen übernommenen Daten/Auffassung und eigener Einschätzung/Argumentation erkennbar

Die Textentwürfe wurden hinsichtlich der benannten Kriterien auf einer vierstufigen Skala eingeschätzt. Die Skala umfasste die Stufen «Hier sind Sie stark», «ok», «Ansätze sind da aber ...» sowie «Hieran müssen Sie arbeiten». Mit der Skala wurde der Versuch unternommen, möglichst sprechende Rückmeldungen zu geben ${ }^{201}$. Zudem erlaubte der Rückmeldebogen die Formulierung offener Bemerkungen, welcher für alle Rückmeldungen genutzt wurde. Die Dokumentation der Einschätzung erfolgte in Form eines handschriftlich ausgefüllten Feedbackbogens, welcher über die Moodle-Foren zur Verfügung gestellt wurde. Zusätzlich wurde der bisherige Textentwurf kommentiert und ebenfalls über das Moodle-Forum zugänglich gemacht. Eine exemplarische Rückmeldung aus der zweiten Phase umfasste entsprechend zwei Dateien (Anhang D.1.3.4).

201 Die Skala erfüllt jedoch nicht den Anspruch, äquidistante Abstände hinsichtlich der Qualität abzubilden. 
Weiterhin wurde Moodle genutzt, um Studierenden Partizipationsmöglichkeiten zur formalen Gestaltung des Wikibooks zu eröffnen. Dies erfolgte in Form von Abstimmungen zur Gestaltung des Inhaltsverzeichnisses (Anhang D.21) sowie zur Berücksichtigung einer geschlechtergerechten Sprache (Anhang D.22). Nach einem Gespräch in der zweiten Präsenzsitzung dieser Projektphase (Kap. 7.2.3.1) wurden in den Moodle-Kursen jeweils zwei Abstimmungen erstellt. Nach dem Ablauf des Umfragezeitraums wurden die Abstimmungsergebnisse der beiden Veranstaltungen summiert und das gemeinsame Ergebnis beiden Seminargruppen zur Verfügung gestellt und thematisiert.

\subsubsection{Interaktionen mit dem Wikibook und der Wikicommunity}

Anlässe zur reflexiven sowie zur gestalterischen Auseinandersetzung mit dem Wikibook erfolgten im Rahmen der Präsenzphasen (Kap. 7.2.3.1) sowie über Abstimmungen über Moodle (Kap. 7.2.3.2). Zudem bestand für die Studierenden im Rahmen dieser Seminarphase die Aufgabe darin, ein Wikibookkapitel zu einem gewählten erziehungswissenschaftlichen Denkwerkzeug zu formulieren. Dafür mussten sie erneut eine Wikiseite für ihr Thema im Rahmen eines spezifischen Kapitels anlegen sowie ihren Text mithilfe der Wikisyntax formatieren.

Auf Basis der Erfahrungen des ersten Seminars wurde zur Erstellung einer neuen Wikiseite die Arbeitsanleitung für das neue Kapitel angepasst, indem der zugehörige Pfad entsprechend verändert wurde. Damit wurde das Ziel verfolgt, Studierenden Orientierungsangebote im Umgang mit dem Wikibook-Editor bereitzustellen. Bis auf wenige Ausnahmen, gelang die Erstellung eigener Wikiseiten durch die studentischen Arbeitsgruppen. Bei Problemen mit Wikisyntax wurden von den Wikibookianern Juetho und Stephan Kulla verschiedene Überarbeitungen vorgenommen. Zum Umgang mit diesen Problemen wurden von Juetho auch Kommentare auf der Diskussionsseite des Wikibooks formuliert ${ }^{202}$. Darüber hinaus wurden Kommentare zu konkreten Wikiseiten formuliert. In deutlicher Sprache werden dabei von Juetho verschiedene Probleme benannt (Abb. 7.7).

202 Zur allgemeinen Gestaltung und Formatierung von Wikibooks beinhalteten diese Kommentare u. a. die Hinweise auf Vorlagen zur Referenzierung verwendeter Literatur sowie zur Textgestaltung hinsichtlich verwendeter Leerzeilen. 


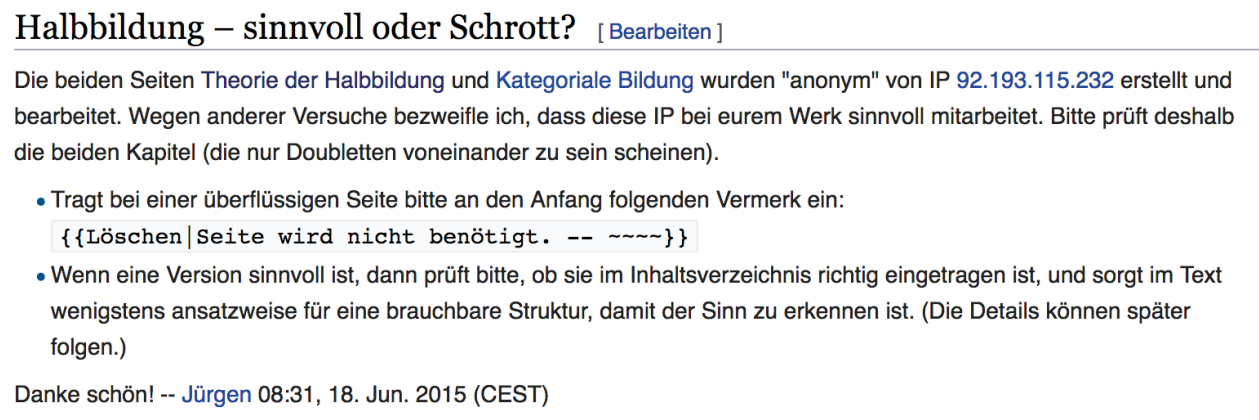

Abb.7.7: Screenshot eines Kommentars auf der Diskussionsseite «Halbbildung» (Anhang E.188)

Als Lehrender war ich für entsprechende Kommentare auch weiterhin dankbar, denn diese stellten eine Unterstützung zur Realisierung des Wikibooks dar, indem sichtbare Fallstricke benannt und zugehörige Unterstützungs- und Orientierungshilfen eröffnet wurden. Zudem konnten die Kommentare sowie die vorgenommenen Bearbeitungen der Mitglieder der Wikicommunity in den Präsenzseminaren erneut thematisiert werden, um die Prinzipien kollaborativer Textproduktion in Wikis zu erläutern. Für die Durchführung der dritten Phase des Seminars sorgten die gemachten Erfahrungen jedoch auch für Zweifel hinsichtlich des formulierten Planung (Kap. 6.6.1.3). Nachdem eine Vielzahl der Studierenden weiterhin unangemeldet Bearbeitungen vornahmen und es weiterhin Schwierigkeiten mit der Wikisyntax gab, erschien mir die individuelle Erstellung von Wikiseiten als Überforderung. Meine Zweifel hinsichtlich der Verschriftlichung der individuellen Analysen und Reflexionen zeigen sich beim folgenden Sprachmemo:

«Und zwar die Frage, inwiefern man das jetzt noch mal verpflichtend macht, das hochzuladen. Ich meine, es war von Anfang gesagt, aber ich bin mir nicht so ganz sicher, ob das für die so produktiv noch mal ist. Oder, was heisst produktiv. Inwiefern das noch mal enorm Arbeit für uns produziert, weil im Prinzip müssten wir die Seiten ein Stück weit vorher vielleicht anlegen und dann die Leute darauf nur schreiben lassen. Vielleicht wäre das eine Variante (kurze Pause), damit irgendwie umzugehen, dass die Studierenden nicht immer irgendwelche Seiten erstellen und die dann (kurze Pause) komplett, also die dann komplett nicht mehr benutzt werden oder umgeleitet werden müssen oder so verschiedene Sachen. Also das ist ein Stück weit das Dilemma» (Sprachmemo 018, 9:35-11:00 min, 10.07.2015).

Neben dem Arbeitsaufwand war das Problem bei der individuellen Erstellung der Wikibeiträge, dass die sich eröffnenden Erfahrungsmöglichkeiten nicht mehr im Seminar thematisiert werden konnten. Die bisher aufgetretenen Schwierigkeiten waren deshalb interessant gewesen, weil sie als authentische Problemstellung in den 
Präsenzseminaren zum Thema gemacht werden konnten. Aufgrund der begrenzten Anzahl an Terminen in der dritten Seminarphase gab es diese Möglichkeit jedoch nicht. Um Situationen zu vermeiden, in denen Studierende an der Veröffentlichung eines Beitrages scheitern, ohne im Rahmen der Präsenzveranstaltung Unterstützung erfahren zu können, wurde die Veröffentlichung der eigenen Analyse im Rahmen des Wikibooks für die Studierenden zur optionalen Möglichkeit erklärt. Die Verschriftlichung der Analyse musste jedoch über Moodle abgegeben werden (Kap. 7.2.4.2). Für die wissenschaftliche Analyse war es interessant, wie viele Studierende diese optionale Möglichkeit wahrnehmen würden (Kap. 7.4.4.3).

\subsubsection{Phase III: Reflexion eigener Vorstellungen}

Die Dokumentation und Reflexion der Praxiserfahrungen der dritten Seminarphase erfolgt erneut hinsichtlich drei gewählter Schwerpunkte: (1.) Erfahrungen während der Präsenzveranstaltungen, (2.) Erfahrungen mit Moodle sowie (3.) Überarbeitungen des Wikibooks.

\subsubsection{Erfahrungen während der Präsenzveranstaltungen}

Die dritte Phase des Seminars umfasste insgesamt drei Präsenztermine. In Adaption der ursprünglichen Zeitplanung (Abb. 7.8) diente die erste Veranstaltung insbesondere der Aufarbeitung der bisherigen Projektergebnisse. Dafür erfolgte zunächst ein Input mit den Ergebnissen der Reviews hinsichtlich der Kriterien zur Begutachtung sowie Kommentare zu den Rückmeldungen. Im Anschluss konnten die Studierenden sich mit den Rückmeldungen auseinanderzusetzen, Überarbeitungspotenziale identifizieren und eine Zeitplanung zur Bearbeitung erstellen. Zum Abschluss der Veranstaltung wurde ein Ausblick auf die weitere Gestaltung der dritten Seminarphase gegeben sowie formale Abgabekriterien und Abgabetermine konkretisiert (Kap. 7.2.4.3).

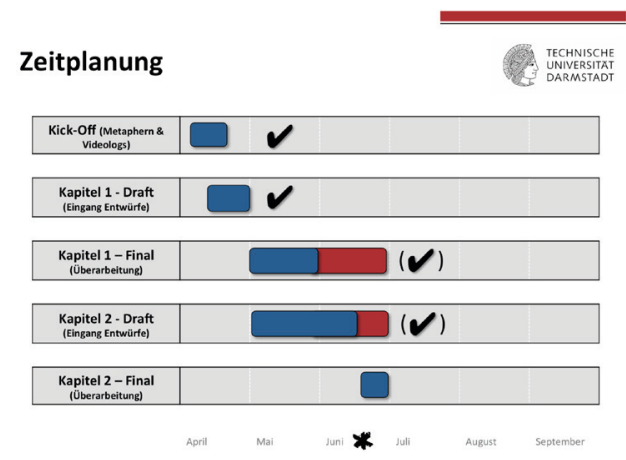

Abb. 7.8: Folie zur adaptierten Zeitplanung zur Fertigstellung der studentischen Arbeitsprodukte aus der zweiten Seminarsitzung (eigene Darstellung). 
Der zweite Präsenztermin widmete sich den Möglichkeiten der Metaphernanalyse mithilfe eines erziehungswissenschaftlichen Denkwerkzeugs. Dafür wurde zunächst der Sinn dieses Vorgehens erläutert und anschliessend zwei Möglichkeiten zum Vorgehen exemplarisch veranschaulicht (Abb. 7.9). In einem alltagssprachlichen Zugang wurde der Analysebegriff in Anlehnung an die Wikipedia-Definition ${ }^{203}$ in drei Schritten unterschieden: (1.) Systematische Zerlegung, (2.) Ordnung der Bestandteile sowie (3) Auswertung bzw. Beurteilung. Als exemplarische Kriterien zur Zerlegung der Gruppenmetaphern wurden zwei Varianten unterschiedenen: (a) Kriterien der Formatvorlage, z. B. die Frage nach der normativen Dimension, (b) Strukturmerkmale der erziehungswissenschaftlichen Denkmodelle, z. B. die Frage nach dem Entscheidungsfeld des Mediums im Berliner Modell nach Heinmann (1962). Im Anschluss daran konnten die Studierenden individuell zwischen zwei Möglichkeiten zur weiteren Nutzung der Präsenz entscheiden: (1.) Gemeinsames Ausprobieren einer Analyse an einem fremden Beispielmaterial und Diskussion, (2.) Beginn mit der (individuellen) Analyse der eigenen Metapher unter einer selbstgewählten Fragestellung.
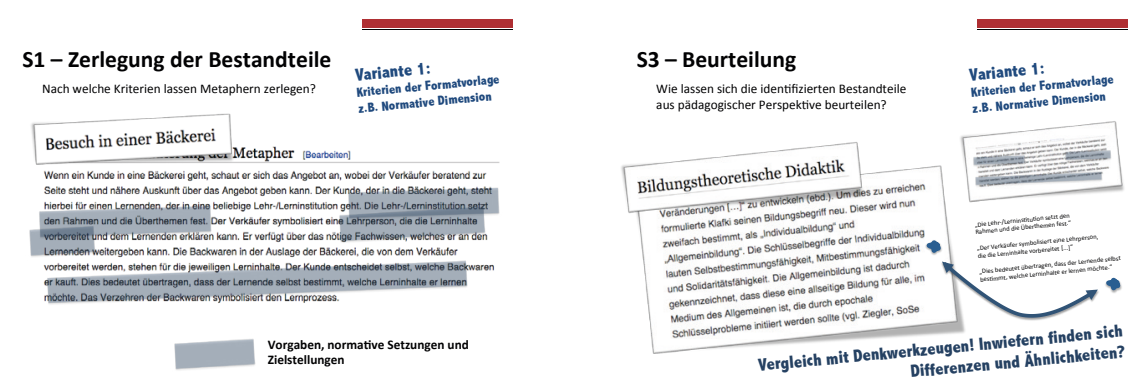

Abb. 7.9: Folien zur exemplarischen Erläuterung der Reflexion der erstellten Metaphern (eigene Darstellung).

Die Detailplanung zum Seminarabschluss erlebte ich als spannungsvoll. Einerseits wollte ich die Veranstaltung aus der Perspektive als Forscher im Rückblick auf die letzten Monate evaluieren und mit den Studierenden diskutieren. Anderseits sah ich aus der Perspektive des Lehrenden die Notwendigkeit, jeweils ein ausführliches Feedback zu den individuellen Analysen der Studierenden, deren Qualität unterschiedlich ausfiel, bereitzustellen. Neben einem inhaltlichen Feedback erschien die Reflexion des Medienhandelns und des Umgangs mit dem Wikibook sinnvoll aber unter den gegebenen zeitlichen Rahmenbedingungen kaum realisierbar. Um die verschiedenen Aspekte auszubalancieren, entstand folgende Strukturierung zur Durchführung des letzten Präsenztermins.

203 Der Auszug lautete «Eine Analyse [...] ist eine systematische Untersuchung, bei der das untersuchte Objekt oder Subjekt in Bestandteile (Elemente) zerlegt wird und diese anschließend geordnet, untersucht und ausgewertet werden» http://de.wikipedia.org/wiki/Analyse (24.04.2014). 
1. Rückblick - Kommentare zu den gesetzten und erreichten Zielen: Bezugnehmend auf die erste Veranstaltung wurde erläutert, inwiefern die formulierten Ziele aus der Perspektive des Lehrenden durch die Aktivitäten des Seminars erreicht werden konnten (Abb. 7.10). Zudem wurde das Wikibook über eine Exportfunktion als PDF-Datei gespeichert und ausgedruckt, um dieses als haptisch wahrnehmbares Produkt zugänglich zu machen.

2. Ausblick(e) zur Erbringung der Studienleistungen und zur Weiterentwicklung des Projektes: Um Studierenden einen Einblick in die Einschätzung ihrer Analyse zu geben, wurden die Kriterien zur Einschätzung der Angemessenheit vorgestellt und an einer Analyse exemplarisch erläutert. Dafür wurden die einzelnen Kriterien diskutiert und abschliessend die Beurteilungsstufen von «Zur Veröffentlichung empfohlen» bis «Intensive Überarbeitung für Studienleitung notwendig» erklärt (Anhang D.28). Als Ausblick auf Fortführung des Projektes wurden zudem die folgenden Aspekte angesprochen: (a) Rückmeldungen aus Portugal, (b) Bitte um Feedback zu den Kapiteleinleitungen sowie (c) weitere Projektschritte über das Semester hinaus.

3. Eure Perspektive - Mut zur Kritik und zur Mitgestaltung - entwicklungsorientierte Bildungsforschung: Die Erhebung der Perspektive der Studierenden erfolgte in drei Phasen bzw. in drei unterschiedlichen Formaten: (a) Kurzfragebogen zur Erfassung eines breiten Spektrums von individuellen Einschätzungen zum Erleben des Seminars (b) Gruppengespräche mit Moderationskarten zu den TOPs, FLOPs und blinde Flecken des Seminars sowie (c) eine offene Abschlussrunde im Plenum.

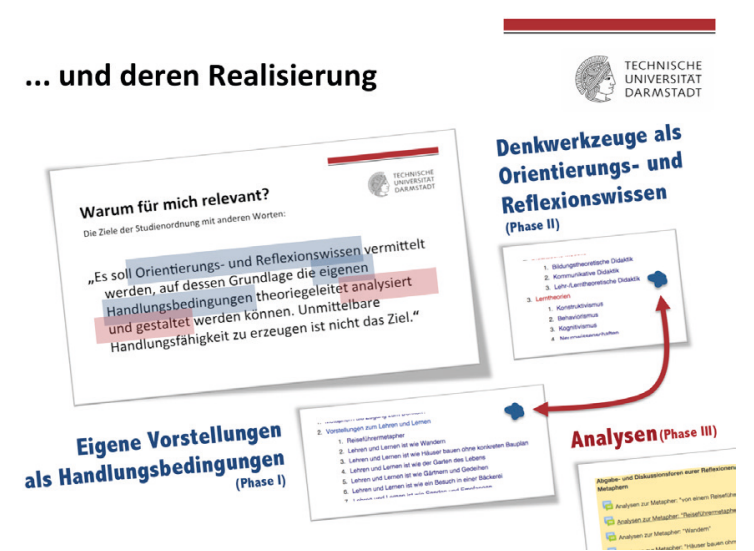

Abb. 7.10: Folie zur Erläuterung der Beziehung zwischen Zielstellung und Aktivitäten des Seminars (eigene Darstellung).

Mit der Verwendung eines Evaluationsbogens konnten Studierende zu konkreten Aspekten der Veranstaltung befragt werden. In den offenen Gesprächsrunden konnten Studierende ihre eigenen Themen zum Ausdruck bringen. Die im Rahmen 
des dritten Schrittes erhobenen Daten bilden die empirische Basis der Veranstaltungsevaluation. Sie eröffnen einen Einblick in die studentische Wahrnehmung und Bewertung des Seminars auf zwei Ebenen.

\subsubsection{Erfahrungen mit Moodle}

Mit dem Einsatz von Moodle wurden in der dritten Projektphase die folgenden Ziele adressiert: (1.) Dokumentation der Präsenzveranstaltung, (2.) Bereitstellung von Orientierungsangeboten, (3.) Schaffung einer Abgabemöglichkeit für die zu erbringenden Leistungen sowie (4.) Formulierung von Rückmeldungen zu den Textentwürfen der Studierenden geben. Zur Orientierung wurde zudem der Sinn der dritten Projektphase für die Studierenden in einem Textfenster im Moodlekurs expliziert ${ }^{204}$.

In dieser Phase erfolgte die Bereitstellung von digitalen Orientierungsangeboten in Form von Dokumenten zu den Präsenzveranstaltungen (Foliensatz und Übungsmaterial) sowie durch das Bereitstellen der Reviewkriterien in Form des Rückmeldebogens für die zu schreibenden Analysen. Auf Basis der bisherigen Erfahrungen mit dem Wikibook (Kap. 7.2.3.3) wurde entschieden, die Aufgabenstellung zu verwenden: «Verfassen Sie eine individuelle Analyse/Reflexion einer von Ihnen ausgewählten Metapher unter einer spezifischen Fragestellung auf Basis einer erziehungswissenschaftlichen Theorie/eines Modells. Die zu analysierende Metapher sowie das Denkwerkzeug als Analysebrille können aus den Themen und Beiträgen des Wikis frei gewählt werden. Der Umfang der Analyse sollte 3000 Zeichen (ohne Leerzeichen) bzw. eine DIN A4 Seite nicht überschreiten. Posten/Veröffentlichen Sie Ihre Analyse bis zum Freitag, den 10. Juli 2015 (um 23:55 Uhr) in den Moodle-Foren. Bitte benennen Sie Ihre Beiträge wie folgt: ‘[Metaphername] aus der Perspektive von [Theorie/Modellname]. Das Verfassen einer angemessen Metaphernanalyse ist Voraussetzung für den Erwerb einer Studienleistung und kann als Vorbereitung für die Modulprüfung betrachtet werden».

Das Feedback für die Studierenden zum dritten Teil der Studienleistung erfolgte, wie die Abgabe der Studienleistungen, über Moodle-Foren (Tab. 7.5). Im Vergleich zu den vorherigen Gruppenleistungen waren in dieser Seminarphase deutlich mehr

204 Die Formulierung lautete: «Der Sinn der dritten Phase ist es, mithilfe der didaktischen sowie lern- und bildungstheoretischen Überlegungen die eigenen Vorstellungen neu durchdenken zu können. Eine entsprechende reflexive Auseinandersetzung mit den eigenen Vorstellungen ermöglicht einen Einblick darin, inwiefern die eigenen Vorstellungen bereits durch erziehungswissenschaftliche Grundgedanken geprägt sind oder inwiefern bestimmte Facetten im Kontext von Lehren, Lernen und Bildung bisher gar nicht wahrgenommen werden können. Damit bietet sich die Möglichkeit, eure Vorstellungen über Lehren und Lernen (welche mithilfe von Metaphern bereits versprachlicht wurden) zu hinterfragen. Durch die Diskussion und Verschriftlichung eurer Gedanken werden ferner eure analytischen Fähigkeiten sichtbar und für Feedback zugänglich». 
Rückmeldungen zu formulieren. Im Seminar wurden «Lehren und Lernen metaphorisch verstehen» wurden 26 verschiedene Analysen und Reflexionen eingereicht. Im Seminar «Bildung metaphorisch verstehen» waren es 13 verschiedene Analysen und Reflexionen. Wie in der Aktivitätsübersicht der Moodle-Foren (Tab. 7.5) sichtbar wird, wurden von den Lehrenden 45 Antworten auf studentische Threads formuliert. 44 der 45 Antworten waren schriftliche Rückmeldungen für Beiträge von Studierenden $^{205}$. Aufgrund der Anzahl an zu begutachtenden studentischen Beiträgen konnten die Rückmeldungen erst im August veröffentlicht werden.

\begin{tabular}{|l|l|l|l|l|}
\hline & \multicolumn{2}{l}{} & \multicolumn{2}{l|}{ Studierende } \\
\cline { 2 - 6 } & Threads & Antworten & Threads & Antworten \\
\hline Metapher zum Lehren und Lernen 1 & 0 & 1 & 1 & 1 \\
\hline Metapher zum Lehren und Lernen 2 & 0 & 1 & 2 & 1 \\
\hline Metapher zum Lehren und Lernen 3 & 0 & 5 & 4 & 1 \\
\hline Metapher zum Lehren und Lernen 4 & 0 & 2 & 2 & 2 \\
\hline Metapher zum Lehren und Lernen 5 & 0 & 9 & 6 & 5 \\
\hline Metapher zum Lehren und Lernen 6 & 0 & 4 & 5 & 1 \\
\hline Metapher zum Lehren und Lernen 7 & 0 & 6 & 6 & 1 \\
\hline Metapher für Bildung 1 & 0 & 6 & 4 & 5 \\
\hline Metapher für Bildung 2 & 0 & 2 & 2 & 1 \\
\hline Metapher für Bildung 3 & 0 & 2 & 1 & 1 \\
\hline Metapher für Bildung 4 & 0 & 7 & 5 & 5 \\
\hline
\end{tabular}

Tab. 7.5: Aktivitätsübersicht der Moodle-Foren der dritten Seminarphase über erstellte Threads und Antworten im Auswertungszeitraum vom 24.06.2015 bis zum 30.09.2015.

Die Rückmeldungen erfolgten in Form digitaler Kopien eines ausgefüllten Feedbackbogens sowie handschriftlich annotierter Texte der Studierenden. Als zusammenfassende Einschätzung wurde zwischen vier Kategorien unterschieden: (1.) Zur Veröffentlichung empfohlen, (2.) Nach leichten Überarbeitungen zur Veröffentlichung empfohlen, (3.) Nach intensiven Überarbeitungen zur Veröffentlichung empfohlen sowie (4.) Intensive Überarbeitungen für Studienleistung notwendig. Die Formulierung der Kategorien erfolgte in Anlehnung an Bewertungsbögen von Zeitschriften mit Peer-Review und sollte die Studierenden ermuntern, sich individuell mit dem Wikibook auseinanderzusetzen bzw. zu erkennen, ob eine Überarbeitung zur Erbringung der Studienleistung notwendig ist. Ferner wurde den Studierenden im Rahmen der Forenposts erläutert, wie die Rückmeldungen verstanden werden

205 Verschiedene studentische Beiträge erhielten mehr als eine Rückmeldung. 
können. Zudem wurde eine kleinschrittige Anleitung ${ }^{206}$ zur Erstellung eines Wikibeitrags beigefügt. Mit der Anleitung wurde das Ziel verfolgt jenen Studierenden einen Zugang zur Nutzung des Wikibooks zu ermöglichen, welche in ihren Arbeitsgruppen bis dahin nicht mit der Erstellung einer Wikiseite in Kontakt gekommen waren.

\subsubsection{3 Überarbeitungen des Wikibooks}

Die Interaktionen mit dem Wikibook umfasste in der dritten Seminarphase u. a. Überarbeitungen der Hauptseite des Wikibooks und früher Gruppenleistungen von Studierenden, einen Austausch mit Mitgliedern der Wikicommunity sowie die Veröffentlichung einzelner Kapiteleinleitungen und individueller Analysen der Studierenden. So wurde auf Basis der getroffenen Abstimmung zur Gestaltung der Startseite des Wikibooks (Anhang D.21) das Layout der Hauptseite des Wikibooks überarbeitet. Während eine bis dahin sichtbare Arbeitsfassung die verschiedenen Beiträge ohne Nummerierung auflistete, wurde mit der Überarbeitung des Inhaltsverzeichnisses auch eine Nummerierung der Kapitel eingeführt (Abb. 7.11).
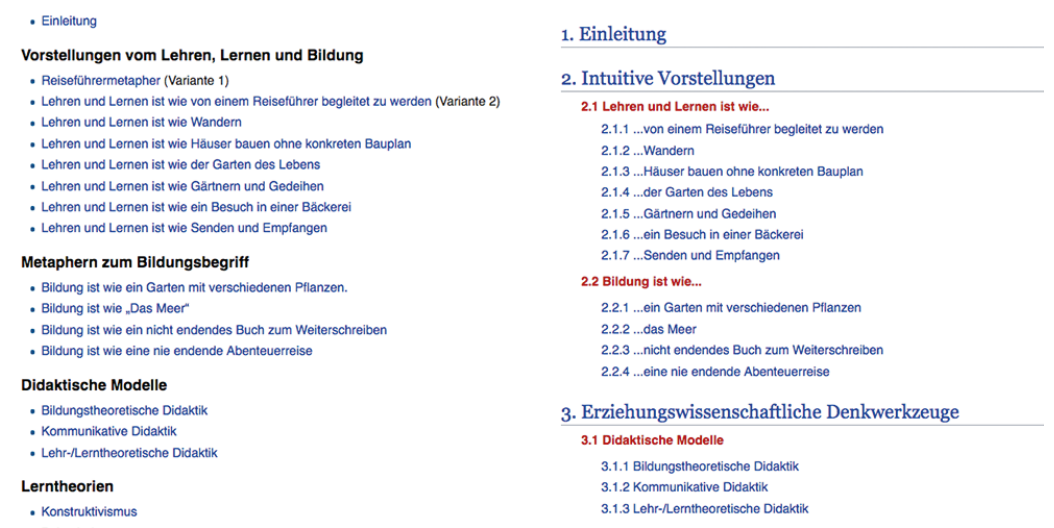

Abb. 7.11: Screenshot zur Formatierung des Inhaltsverzeichnisses des Wikibooks vor der Überarbeitung am 03.07.2015 (links) und nach der Überarbeitung am 09.07.2015 (rechts).

Im Zusammenhang mit der Überarbeitung des Inhaltsverzeichnisses erfolgte die Veröffentlichung von Einführungen zu den jeweiligen Kapiteln. Diese Einführungen sollten den Studierenden sowie den externen Leserinnen und Leser die Möglichkeit eröffnen, den Aufbau der jeweiligen Kapitel sowie dessen Funktion nachvollziehen zu können. Gemessen an zeitlichen Struktur der Seminare erfolgte die Veröffentlichung dieser Kapiteleinleitungen für die Studierenden verhältnismässig spät. Zwar konnte auf die Texte im Rahmen der Präsenzveranstaltungen verwiesen werden,

206 Die Anleitung beinhaltete die folgenden Aspekte: (1.) Rufe diese Seite [Platzhalter für eine Seite im Wiki-book] auf, (2.) Gehe auf «Bearbeiten», (3.) Schreibe eine Überschrift für deine Analyse (Level 2, also $==$ Überschrift ==) (4.) Kopiere deinen Text ein, (5.) Prüfe mit Vorschau was passiert, (6.) Klicke auf Seite speichern. 
allerdings blieb keine Zeit deren Qualität kritisch zu diskutieren. Diese Problematik wird auch im Rahmen der Modifikation des Entwurfs aufgegriffen (Kap. 9.2.3). Eine Veröffentlichung der individuellen studentischen Analysen erfolgte gemäss der Erfahrungen der zweiten Seminarphase sowie der vorgenommenen Planungskorrektur (Kap. 7.2.3) auf freiwilliger Basis. Dabei zeigte sich, dass nur wenige Studierende dieses Angebot wahrnahmen (Kap. 7.4.4.3).

\subsection{Durchführung der empirischen Analysen}

Das Ziel der folgenden Ausführungen ist die transparente Darstellung des Vorgehens zur Erhebung von Daten sowie deren Analyse für die erste Iteration im Sommersemester 2015. Auf Basis des geplanten Forschungsdesigns (Kap. 3) wird das Vorgehen zur Datenerhebung sowie zur Durchführung der empirischen Analysen vorgestellt. Dieses Vorgehen umfasst die Realisierung strukturierender Inhaltsanalysen von Interviews mit Studierenden (Kap. 7.3.1), die Durchführung einer qualitativen und quantitativen Veranstaltungsevaluation (Kap. 7.3.2), die analytische Betrachtung studentischer Seminarprodukte in Form von Metaphernanalysen (Kap. 7.3.3) und statistische Auswertungen der Wikibook-Interaktionen (Kap. 7.3.4).

\subsubsection{Strukturierende Inhaltsanalyse der Interviews}

Die Durchführung der Interviews erfolgte mit dem Ziel Erkenntnisse darüber zu gewinnen, welche Erfahrungen Studierende im Umgang mit dem Wikibookprojekt machen (Kap. 3.2.1.2) und wie sie die Seminargestaltung und die Lernsituationen zum Lernen mit digitalen Medien erleben und bewerten (Kap. 3.2.1.3). Einen Einblick in die studentischen Perspektiven zu diesen Fragen zu gewinnen, erschien hilfreich, um beurteilen zu können inwiefern die geplanten und realisierten Lehrhandlungen angemessen waren, um die geplanten Lernhandlungen der Studierenden zu unterstützen.

Die Interviews wurden im Zeitraum vom 22. Juni bis zum 6. Juli 2015 durchgeführt ${ }^{207}$. Der Interviewzeitraum entsprach dem Abschluss der zweiten Seminarphase bzw. dem Beginn der dritten Seminarphase. So konnten die Studierenden zu diesem Zeitpunkt über die Erfahrungen der ersten zwei Seminarphasen sowie über die zu erstellenden Seminarprodukte in Gruppenarbeit sprechen. Die Anfrage der Studierenden zur Durchführung eines Interviews erfolgte im Plenum sowie in den Arbeitsgruppen der Präsenzveranstaltungen (Kap. 3.3.2). Zudem wurde eine Anfrage über das Nachrichtenforum der Moodle-Kurse an die Teilnehmerinnen und Teilnehmer versendet. Die Teilnahme an den Interviews war für die Studierenden freiwillig.

207 In der ersten Woche wurden bereits die ersten zehn Interviews durchgeführt. Aufgrund einer Terminkollision erfolgte ein Interview mit einer Woche Verspätung. 
Im angegebenen Zeitraum konnten elf Interviews mit Studierenden realisiert werden (Tab. 7.6). Die Interviewdauer variierte zwischen 18 und 43 Minuten und betrug im Mittel etwa 26 Minuten. Die elf Teilnehmerinnen und Teilnehmer lassen sich auf Basis soziodemografischer Angaben wie folgt beschreiben: Etwa Dreiviertel der Befragten waren Lehramtsstudierende, ein Viertel der befragten Personen studierte Pädagogik (BA). Im Mittel waren die Studierenden im fünften Fachsemester und 25 Jahre alt. Neun Teilnehmerinnen gaben an weiblich zu sein, drei Teilnehmer der Gruppengespräche waren nach eigener Auskunft männlich. Von den elf Studierenden belegten sechs Studierende die Veranstaltung «Lehren und Lernen metaphorisch verstehen» und drei Studierende die Veranstaltung «Bildung metaphorisch verstehen». Zwei Studierende absolvierten beide Veranstaltungen.

\begin{tabular}{|l|l|l|l|l|l|l|l|l|l|l|}
\hline \multicolumn{2}{|l|}{ Studiengang } & \multicolumn{3}{l|}{ Fachsem. } & \multicolumn{2}{l|}{ Alter } & \multicolumn{2}{l|}{ Geschlecht } & \multicolumn{2}{l|}{ Seminar } \\
\hline Lehramt & Pädagogik & M & SD & M & SD & männl. & weibl. & Didaktik & Bildung \\
\hline 8 & 3 & 5 & 3 & 25 & 8 & 2 & 9 & 7 & 4 \\
\hline
\end{tabular}

Tab. 7.6: Beschreibung des Interviewsamples im Sommersemester 2015.

Zur Vorbereitung der Interviewanalyse erfolgte eine Transkription der Aufnahmen (Kap. 3.3.2). Die erstellten Transkripte stellten die Datenbasis für die inhaltsanalytische Auswertung dar (Anhang B.6). Wie im Kapitel "Strukturierende Inhaltsanalyse der Interviews» (Kap. 3.4.1.1) beschrieben, erfolgte zunächst eine fallbezogene Darstellung der zentralen und auffälligen Aspekte für jedes Interview. Als Orientierungspunkte für diese fallbezogene Erkundung sowie zur Formulierung der Fallzusammenfassung stand insbesondere die Auseinandersetzung mit dem Wikibook sowie die Zusammenarbeit in den Seminargruppen im Fokus. Es entstanden elf Kurztitel als charakteristische Bezeichnungen der Interviews (Anhang B.6.1). Diese lauteten u. a. «Die engagierte Wikinutzerin bzw. der engagierte Wikinutzer», «Die Oldschoolerin bzw. der Oldschooler» sowie «Die Person, die zum ersten Mal etwas im Internet veröffentlicht».

Aufgrund des begrenzten zeitlichen Rahmens zur Auswertung der Daten bis zur Überarbeitung des Entwurfs wurde eine kategorienbasierte Auswertung nach Kuckartz et al. $(2008,36)$ gewählt. Die Durchführung der Auswertung orientierte sich zudem am Vorgehen von Mayring $(1990,2000,2002)$ zur strukturierenden Inhaltsanalyse (Kap. 3.4.1.1). Auf Basis der fallbasierten Erkundung des Interviews sowie unter Berücksichtigung der für die Interviews relevanten Fragestellungen (Kap. 3.2.1.3, 3.2.1.2) wurde in Zusammenarbeit mit einem studentischen Mitarbeiter ein Kodierleitfaden mit vier Hauptkategorien entworfen (Anhang A.1.3). Die Bezeichnung der Kategorien sowie die zugehörigen Definitionen lauten:

- K1 Einstellung gegenüber der Projektidee eines kollaborativen Wikibookprojektes: Diese Kategorie umfasst Aussagen zu Einstellungen, Interessen und Ängsten 
gegenüber der Projektidee im Seminarkontext gemeinsam an einem Wikibook zu schreiben (z. B. zur Erstellung konkreter Texte, zur Veröffentlichung bzw. zum öffentlichen Schreiben).

- K2 Individuelle Vorerfahrungen und Kenntnisse: Diese Kategorie umfasst Aussagen zu eigenen Erfahrungen mit ähnlichen Projekten, Selbstzuschreibungen des eigenen Kenntnisstandes hinsichtlich der Nutzung von Wikis, dem Schreiben von Texten sowie der inhaltlichen Auseinandersetzung.

- K3 Vorgehen zur Erstellung von Wikibookbeiträgen in Gruppenarbeit: Diese Kategorie umfasst Beschreibungen und Reflexionen zum Vorgehen der Studierenden in der ersten und zweiten Seminarphase zur Selbst- und Gruppenorganisation, zum Schreiben an einem gemeinsamen Text in einer Gruppe sowie zum Umgang mit dem Wikibook.

- K4 Wahrnehmung der Wikibook-Community: Diese Kategorie umfasst Beschreibungen und Reflexionen zu gemachten Erfahrungen mit der Wikibook-Community, z.B. im Lesen anderer Wikibooks bzw. anderer Texte neben dem eigenen Beitrag, die Auseinandersetzung mit den Kommentaren und Überarbeitungen von anderen etc.

Neben der Bezeichnung und Definition der Hauptkategorien beinhaltet der vollständige Kodierleitfaden in Anlehnung an Mayring $(1990,2000)$ jeweils Ankerbeispiele in Form von Interviewzitaten des vorliegenden Materials. Zudem wurden die Kategorien «K3 Vorgehen zur Erstellung von Wikibookbeiträgen in Gruppenarbeit» sowie "K4 Wahrnehmung der Wikibook-Community» in weitere Subkategorien differenziert (Anhang A.1.3). Hinsichtlich der zu bestimmenden Analyseeinheit wurde entschieden, dass Sinneinheiten mindestens im Umfang eines Satzes bzw. vorzugsweise im Umfang eines Absatzes zu kodieren sind. Bei Bedarf können bzw. müssen auch mehrere Absätze sowie die Frage des Interviewers kodiert werden. Das zentrale Kriterium zur Auswahl des Textumfangs ist, dass die kodierte Textstelle «für sich allein ausreichend verständlich ist» (Kuckartz et al. 2008, 39). Ferner wurde festgehalten, dass Mehrfachkodierungen einer Textstelle möglich sind. Die interviewten Personen können Aussagen getroffen haben, die sich mehreren Kategorien zuordnen lassen, z. B. weil sie ihre spezifischen Einstellungen gegenüber Wikibooks (K1) aufgrund von Vorerfahrungen (K2) begründen. Entsprechende Sinneinheiten sind mit allen zutreffenden Kategorien zu kodieren.

\subsubsection{Qualitative und quantitative Veranstaltungsevaluation}

Um einen Einblick in die studentische Perspektive auf die Seminargestaltung sowie die verschiedenen Lernsituationen zu erhalten (Kap. 3.2), wurde zum Seminarabschluss eine Veranstaltungsevaluation durchgeführt. Die Evaluation umfasste eine 
quantitative Dimension durch die Verwendung eines modifizierten Evaluationsbogens der TU Darmstadt sowie eine qualitative Datenerhebung in Form von offenen Gesprächsrunden mit studentischen Kleingruppen. Entsprechend der chronologischen Reihenfolge der Datenerhebung wird im Folgenden zunächst das Vorgehen der Erhebung und Auswertung des Evaluationsbogens skizziert sowie die zugehörige Stichprobe charakterisiert (Kap. 7.3.2.1). Anschliessend wird die Durchführung der strukturierten Gruppengespräche beschrieben, die zugehörige Gelegenheitsstichprobe charakterisiert und das Vorgehen kommentiert (Kap. 7.3.2.2).

\subsubsection{Anpassung und Auswertung des Evaluationsbogens}

Die Verwendung eines quantitativen Evaluationsbogens erfolgte mit dem Ziel, die Seminargestaltung aus studentischer Perspektive bewerten und einschätzen zu lassen. Im Fokus standen die studentische Perspektive zur Gesamteinschätzung des Seminars, die Bewertung konkreter Lernsituationen und Lehrhandlungen (Kap. 3.2.1.3) sowie die Einschätzung spezifischer Aspekte zum Erleben des Wikibooks (Kap. 3.2.1.2).

Zur Entwicklung eines dafür geeigneten Erhebungsinstrumentes wurde der standardisierte Evaluationsbogen für Seminare vom Fachbereich Humanwissenschaften an der TU Darmstadt erweitert und angepasst. Neben der Angabe demografischer Daten (Alter, Geschlecht, Studiengang, Fachsemester) zur Beschreibung der Stichprobe wurden die Studierenden in Übereinstimmung zum Evaluationsbogen der TU Darmstadt zu ihrer Einschätzung bezüglich der Organisation und der Transparenz der Lehrveranstaltung (8 Items), zum Engagement der Lehrperson und der Aktivität der Studierenden (8 Items), zu den Anforderungen und zum Arbeitsaufwand (4 Items) sowie zum Lernerfolg und zur eigenen Zufriedenheit befragt ( 6 Items). Verschiedene Items des Evaluationsbogens konnten für die eigene Evaluation übernommen werden. Zur Einschätzung des Engagements der Lehrperson und der Aktivität der Studierenden wurden z. B. die zwei Items «Für einen inhaltlichen Austausch im Seminar ist genug Zeit.» sowie «Am Seminargeschehen habe ich mich beteiligt.» direkt übernommen. Weitere Aussagen wurden in Anlehnung an den Entwurf des Seminars (Kap. 6) konkretisiert bzw. differenziert. Die Aussage des Evaluationsbogens «Die Lehrperson regt uns gezielt zur eigenen Mitarbeit / zum Mitdenken an» wurde für den Fragebogen der Fallstudie beispielsweise für die Präsenzveranstaltungen konkretisiert. Zudem wurden zwei weitere Frage ergänzt, welche sich insbesondere den Rückmeldungen über Moodle widmeten: (1.) «Die schriftliche Rückmeldung für unsere Metapher war für mich nachvollziehbar.» sowie (2.) «Die schriftliche Rückmeldung zu unserem Text der zweiten Phase (Denkwerkzeuge) war für mich nachvollziehbar.» Entsprechende Anpassungen am Evaluationsbogen wurden für alle Items vorgenommen, für die unter Berücksichtigung des Seminarentwurfs - eine Konkretisierung sinnvoll war. 
Auf Basis der fallbezogenen Erkundung der Interviews (Kap. 7.3.1) wurden zwei weitere Themenkomplexe hinzugefügt: (1.) Erfahrungen mit Wikibooks und Medieneinsatz in der Lehrveranstaltung sowie (2.) Einstellung zu medialen Lehr- und Lernkonzepten. Die Entwicklung von Aussagen zu den entsprechenden Themen orientierte sich an auffälligen Aussagen in den Interviews. So zeigte sich bei der fallbezogenen Erkundung und der Erstellung der zugehörigen Kurzprofile beispielsweise, dass sich zwei Studierende «Die Oldschoolerin bzw. der Oldschooler» sowie «die bzw. der Desinsteressierte» sehr distanziert zur Verwendung digitaler Medien in Seminaren geäussert hatten. Beide Studierende hatten sich u. a. positiv darüber geäussert, dass die Bearbeitung des Wikibooks in ihren Seminargruppen von jeweils anderen Studierenden übernommen worden waren und sie sich mit der Nutzung des Wiki-Editors nicht beschäftigen mussten. Für den Themenbereich «Erfahrungen mit Wikibooks und Medieneinsatz in der Lehrveranstaltung» wurden in Auseinandersetzung mit den benannten und weiteren Interviewprofilen $u$. a. die folgenden drei Items formuliert: «Das Veröffentlichen der Texte im Wikibook habe ich übernommen», «Die Bedienung des Wiki-Editors war für mich einfach» sowie «Das Seminar bot genügend Unterstützung zur Nutzung von Wikibooks».

Die Befragung der Studierenden erfolgte im Rahmen der letzten Präsenzveranstaltung in gedruckter Form ( $N=17)$ sowie als Onlinebefragung ${ }^{208}(\mathrm{~N}=2)$. Mit der Befragung der Studierenden mit Hilfe eines Evaluationsbogens als Online- und Präsenzbefragung wurde das Ziel verfolgt, möglichst viele Teilnehmerinnen und Teilnehmer zu erreichen, was allerdings nur begrenzt gelang. Die Zusammenstellung der Teilnehmerinnen und Teilnehmer kann als Gelegenheitsstichprobe bezeichnet werden (Kromrey 2009, 264 f.), die nicht auf einem zufallsgesteuerten Auswahlverfahren basiert. Die 19 Teilnehmerinnen und Teilnehmer der quantitativen Befragung lassen sich auf Basis soziodemografischer Angaben wie folgt beschreiben: Über zwei Drittel waren Lehramtsstudierende und fast ein Drittel der Teilnehmerinnen und Teilnehmer studierten Pädagogik. Im Mittel waren die Studierenden im vierten Fachsemester und 24 Jahre alt. 14 Teilnehmerinnen gaben an weiblich zu sein und fünf Teilnehmer der Gruppengespräche nach eigener Auskunft männlich (Tab. 7.7).

\begin{tabular}{|l|l|l|l|l|l|l|l|l|l|}
\hline \multicolumn{2}{|l|}{ Studiengang } & \multicolumn{3}{l|}{ Fachsem. } & \multicolumn{2}{l|}{ Alter } & \multicolumn{2}{l|}{ Geschlecht } & \multicolumn{2}{l|}{ Seminar } \\
\hline Lehramt & Pädagogik & M & SD & M & SD & männl. & weibl. & Didaktik & Bildung \\
\hline 13 & 6 & 4 & 3 & 24 & 6 & 5 & 14 & 12 & 7 \\
\hline
\end{tabular}

Tab. 7.7: Beschreibung der Stichprobe der quantitativen Evaluation im Sommersemester 2015.

208 Die Erstellung der Online-Umfrage erfolgte mit einer eigenen Limesurvey-Installation. Die Bearbeitung der Onlinebefragung konnte innerhalb von sechs Wochen erfolgen. 
Die Evaluationsdaten stammen aus zwei thematisch unterschiedlichen Seminaren, die einen identischen konzeptionellen Aufbau aufweisen (Kap. 6.6). Zur Auswertung der Daten wurde entschieden, die Daten zusammenzufassen und gemeinsam auszuwerten. Die Entscheidung begründet sich zum einen durch das jeweils identische zugrundeliegende Konzept. Ausgenommen der konkreten Inhalte wurden die Studierenden in beiden Seminaren mit den gleichen - inhaltlich adaptierten - Aufgabenstellungen und strukturellen Vorgaben konfrontiert. Zudem fanden seminarübergreifende Abstimmungen zur Gestaltung des Wikibooks statt (Kap. 7.2.3.2 und Anhang D.21). Insofern wird davon ausgegangen, dass die Studierenden hinsichtlich der Beantwortung der Fragen des Evaluationsbogens auf vergleichbare Erfahrungen zurückgreifen konnten. Zum anderen zeigte sich in den Interviews, dass auch innerhalb der Seminare eine hohe Heterogenität zwischen den einzelnen Gruppen des Seminars bestand. Innerhalb der jeweiligen Seminare liessen sich beispielsweise sehr heterogene Strategien hinsichtlich der Gruppenorganisation und des Schreibens identifizieren, welche zwar für das eine Seminar einzigartig erscheinen, aber seminarübergreifend betrachtet häufiger zu identifizieren sind.

Die Auswertung und Präsentation der quantitativen Daten erfolgt mithilfe von Methoden der deskriptiven Statistik (Kap. 3.4.2.1). Die Darstellung der Ergebnisse erfolgt in Form von Häufigkeitstabellen. Für jedes Item wird die Anzahl der gegebenen Antworten (N), der Mittelwerte (M) zur Bestimmung des Schwerpunktes der jeweiligen Häufigkeitsverteilungen sowie der Standardabweichung (SD) als Streuungsmass der Verteilung angegeben. Zur Darstellung zentraler Tendenzen der Antworthäufigkeiten werden in der Verbalisierung der Häufigkeitstabellen zwei Antwortkategorien zusammengefasst. Die Berechnung der statistischen Kennwerte erfolgte mit der Software SPSS.

\subsubsection{Zusammenfassende Inhaltsanalyse strukturierter Gruppengespräche}

Zur Erfassung der studentischen Perspektive wurden zur Ergänzung des quantitativen Fragebogens Gesprächsrunden von Studierenden durchgeführt. In der Realisierung eines offenen Formates wird das Potenzial gesehen, dass Studierende ihre Perspektive zu Themen und Aspekte einbringen können, welche nicht antizipiert wurden (Kap. 3.3.3.1). Zum Umgang mit den methodischen Herausforderungen zur Durchführung von Gruppeninterviews in Seminarkontexten (Kap. 3.3.3.1) - der spezifischen Rolle im Feld, des Umgangs mit der Dynamik mit Gruppendiskussionen sowie der Entscheidung zur Gruppenzusammensetzung - wurde das Konzept der Gruppendiskussion hin zu einer aufgabenorientierten Gesprächsrunde mit Metaplankarten in der letzten Präsenzveranstaltung adaptiert. Um eine möglichst offene Gesprächssituationen zu schaffen, wurde auf die Anwesenheit der Lehrperson verzichtet. 
Um alle Gruppenteilnehmerinnen und -teilnehmer zum Austausch anzuregen und das zuvor skizzierte Problem der Dynamik einer Gruppendiskussion - welche durch den Verzicht auf eine Moderationsperson noch weniger «kontrollierbar» ist wurde die Gesprächssituation durch eine Aufgabe mit Metaplankarten ergänzt. Die Studierenden wurden aufgefordert, ihre «Tops», «Flops» sowie «Blinde Flecken» des Seminars mithilfe von Metaplankarten zu fixieren, diese anschliessend vorzustellen und zu diskutieren ${ }^{209}$. Die Gruppengespräche erfolgten nach dem Ausfüllen des Evaluationsbogens. Die Studierenden hatten die Möglichkeit, die Gesprächsrunden in Sympathiegruppen von drei bis vier Studierenden durchzuführen. Die Raumsituation der ComputerStudienWerkstatt ermöglichte es, dass sich die Gruppen in verschiedene Bereiche zurückziehen konnten. Die Aufnahme der Diskussion erfolgte durch die Studierenden mit Hilfe von zur Verfügung gestellte Tablets.

Die Durchführung erfolgte jeweils im Rahmen der letzten Präsenztermine mit insgesamt 17 Teilnehmerinnen und Teilnehmer. Im Seminar «Lehren und Lernen metaphorisch verstehen» erfolgte eine Gesprächsrunde in drei Gruppen zwischen drei und vier Personen. Im Seminar «Bildung metaphorisch verstehen» erfolgten zwei Gruppengespräche mit jeweils drei Studierende. In der Gesamtbetrachtung der Teilnehmerinnen und Teilnehmer der Gruppengespräche kann die Zusammenstellung ebenfalls als Gelegenheitsstichprobe bezeichnet werden. Die 17 Teilnehmerinnen und Teilnehmer der strukturierten Gesprächsrunden lassen sich auf Basis soziodemografischer Angaben wie folgt beschreiben: Über zwei Drittel waren Lehramtsstudierende und fast ein Drittel der Teilnehmerinnen und Teilnehmer studierten Pädagogik. Im Mittel waren die Studierenden im fünften Fachsemester und 25 Jahre alt. 13 Teilnehmerinnen gaben an weiblich zu sein und vier Teilnehmer der Gruppengespräche nach eigener Auskunft männlich (Tab. 7.8).

\begin{tabular}{|l|l|l|l|l|l|l|l|l|l|}
\hline \multicolumn{2}{|l|}{ Studiengang } & \multicolumn{3}{l|}{ Fachsem. } & \multicolumn{2}{l|}{ Alter } & \multicolumn{2}{l|}{ Geschlecht } & \multicolumn{2}{l|}{ Seminar } \\
\hline Lehramt & Pädagogik & M & SD & M & SD & männl. & weibl. & Didaktik & Bildung \\
\hline 12 & 5 & 5 & 3 & 25 & 7 & 4 & 13 & 11 & 6 \\
\hline
\end{tabular}

Tab. 7.8: Beschreibung des Samples der Gruppengespräche im Sommersemester 2015

Die Aufbereitung der aufgezeichneten Gruppengespräche erfolgte in Anlehnung an Bremer (2001) mithilfe eines modifizierten Leitfadens. Zu jedem Gruppengespräch wurden Kontextinformationen zum Gespräch (u. a. Raum, Zeit, Anzahl der Teilnehmerinnen und Teilnehmer), Informationen auf der Metaebene bzw. zum

209 Wenngleich Gruppendiskussionen mit Metaplankarten selten als Verfahren erziehungswissenschaftlicher oder hochschuldidaktischer Evaluationen eingesetzt werden, liegen mit den forschungsmethodischen Arbeiten von Bremer (2001) und Grell (2006) Möglichkeiten und Chancen eines entsprechenden Vorgehens vor. So hat sich bei Bremer $(2001,79)$ gezeigt, dass ein gegenseitiges Vorstellen der Metaplankarten dazu führen kann, dass sich alle Teilnehmerinnen und Teilnehmer beteiligen und es keine schweigenden Personen mehr gibt (oder geben kann) sowie dass jede neue präsentierte Metaplankarte als Impuls für die Diskussion dient. 
Gruppenprozess (u. a. Teilnahmestatistik, Sequenzprotokoll, Metaplanwände im Überblick) sowie auf der Einzelfallebene (u. a. sozio-demografische Angaben, Metaplankarten und Diskussionsbeiträge) dokumentiert. In der Aufbereitung der aufgezeichneten Gesprächsrunden zeigte sich, dass kaum diskursive Bestandteile in den Aufzeichnungen zu identifizieren waren, bei denen Studierende untereinander Bezug auf ihre Aussagen nahmen (Anhang B.5). Stattdessen kann das so entstandene Material als gruppierte Einzelaussagen von Studierenden zu den positiven, negativen und fehlenden Aspekten der Veranstaltungen verstanden werden.

Zur Analyse dieser Aussagen wurde das Ziel gesetzt, die Aussagen der Studierenden so zusammenzufassen, dass das Spektrum der unterschiedlichen studentischen Perspektiven auf die zwei Seminare in strukturierter Weise sichtbar wird. Zur Auswertung wurde sich für eine qualitative Inhaltsanalyse gewählt. Das Material der Analyse stellten die im Rahmen der Materialaufbereitung erstellten transkribierten Aussagen der Studierenden dar. Die auf den Metaplankarten notierten Statements wurden als sprachliche Aussagen behandelt. Nachvollziehbare Aussagen bzw. Sinneinheiten von Studierenden zur Bewertung und Beschreibung spezifischer Erfahrungen, Situationen und Aspekte des Seminars wurden in der Analyse als «Kodiereinheit» betrachtet, d. h. als kleinster Materialbestandteil der ausgewertet werden darf (Mayring 1990, 49). Entsprechende Aussagen bzw. Sinneinheiten mussten formal mindestens als Teilsätze eines grösseren Satzgefüges zu erkennen sein. Die Durchführung des ersten Reduktionsschritts der zusammenfassenden Inhaltsanalyse erfolgte zunächst gruppenspezifisch mit dem Ziel, möglichst spezifische Aussagen über die Einschätzung der Teilnehmerinnen und Teilnehmer zum Seminar hinsichtlich der positiven, negativen und wünschenswerten Aspekte zusammenzufassen (Anhang B.5.6). Im zweiten Reduktionsschritt wurden die generalisierten Aussagen bzw. vorläufigen Kategorien für das jeweilige Seminar betrachtet. Im dritten Schritt erfolgte die Reduktion seminarübergreifend mit dem Ziel, Einschätzungen von spezifischen Aspekten zum erlebten Wikibookprojekt im Kontext der jeweiligen Seminare zusammenzufassen. Als theoretische Strukturierungshilfe zur Konstruktion und Bündelung von Kategorien dienten die verschiedenen Entscheidungsfelder im Planungsprozess (Kap. 6.6) sowie eine Differenzierung nach didaktischen Handlungsebenen (Kap. 4.2.3).

\subsubsection{Metaphernanalyse studentischer Schreibprodukte}

Im konzeptuellen Entwurf (Kap. 6.4) wurde die lerntheoretische Annahme formuliert, dass sich die Fähigkeiten zur Artikulation und Reflexion von Vermittlungs- und Interaktionsprozessen für pädagogisches Handeln im Unterricht $u$. a. dann (weiter-) entwickeln, wenn sich die Studierenden ihrer eigenen Vorstellungen hinsichtlich der Begriffe Lehren, Lernen und Bildung bewusstwerden und mithilfe von Metaphern verschriftlichen und diskutieren. Auf Basis dieser und weiterer Annahmen wurden 
verschiedene Lernsituationen zur Auseinandersetzung mit selbst entwickelten Metaphern, dessen potenzieller Anregungsgehalt näher bestimmt werden soll, geplant und realisiert (Kap. 6.6.1.1, 7.2.2). Im Fokus stand die Frage, inwiefern die Formulierung expliziter Metaphern potenzielle Diskussions- und Reflexionsanlässe über Lehren und Lernen für Studierende eröffnen kann (Kap. 3.2). Das Ziel der Analyse war es, Erkenntnisse über die sprachlichen Artikulationsmöglichkeiten der Studierenden zur Beschreibung von Lehren und Lernen zu gewinnen sowie metaphorische Muster und Vorstellungen der Studierenden zu rekonstruieren.

Für die Analyse des Anregungsgehaltes der entwickelten Lernsituationen wurden Ad-hoc-Metaphern und Gruppenmetaphern als Datenbasis ausgewählt (Kap. 3.3.4). Die Auswahl dieser Daten erfolgte in Anlehnung an die vorgestellten Untersuchungen von Überzeugungen und Vorstellungen von Lehramtsstudierenden (Kap. 5.4.2) sowie in Anlehnung an Untersuchungen zum Reflexionspotenzial von Metaphern im Kontext universitärer Lehre in der Lehrerinnen- und Lehrerbildung (Kap. 5.4.3). Die Fokussierung des didaktischen Schwerpunktes erfolgte aufgrund forschungspraktischer Grenzen. Im Rahmen des Sommersemester 2015 wurden 22 Ad-hoc-Metaphern zum Lehren und Lernen im Unterricht formuliert und für die Analyse berücksichtigt. Ferner wurden acht Gruppenmetaphern formuliert, über das Wikibook veröffentlicht und im Rahmen der vorliegenden Fallstudie analysiert. Die Seminarprodukte der Studierenden lagen bereits durch die Seminardurchführung digital vor.

Zur Analyse der Materialien wurde in der ersten Fallstudie ein zirkuläres Verfahren gewählt, bei dem in mehreren Iterationen jeweils vier Schritte bearbeitet werden (Kap. 3.4.3). Für die Ad-hoc-Metaphern der Studierenden konnten auf Basis des Vorgehens einerseits gemeinsame Muster, die von mehreren Studierenden artikuliert wurden, markiert werden. Ferner eröffnete sich über die Analyse der Gruppenmetaphern die Möglichkeit, die Veränderung verschiedener Metaphern durch die Aktivitäten der Gruppenarbeiten in den Blick zu nehmen. Die Interpretation der Ergebnisse erfolgte auf Basis der beschriebenen Heuristiken (Kap. 3.4.3).

\subsubsection{Deskriptive Statistik der Versionsgeschichte}

Im Zeitraum der ersten Fallstudie wurden vom 1. April 2015 bis zum 30. September 2015 insgesamt 34 Wikiseiten für das Wikibook «Lehren, Lernen und Bildung metaphorisch verstehen» erstellt. Für alle 34 Wikiseiten erfolgte eine Analyse der Versionsgeschichte für die drei unterschiedlichen Seminar- bzw. Auswertungsphasen. Der erste Auswertungszeitraum erfolgte vom Semesterbeginn bis zum Abgabedatum des ersten Schreibproduktes für das Wikibook bzw. vom 1. April 2015 bis 03. Mai 2015. Der zweite Auswertungszeitraum begann am 04. Mai 2015 und endete zum Abgabetermin der Textprodukte für die erziehungswissenschaftlichen Denkwerkzeuge am 23. Juni 2015. Der dritte Auswertungszeitraum erfolgte bis zum Ende des Semesters vom 24. Juni 2015 bis zum 30. September 2015. 
Neben der Startseite umfasste das Wikibook 30 inhaltliche Wikiseiten, welche in fünf verschiedene Kapitel strukturiert sowie drei Diskussionsseiten die zur Startseite sowie zu den Beiträgen der Studierenden erstellt wurden. Die Einleitung des Wikibooks wurde von mir als Lehrender erstellt und stellt das erste Kapitel des Wikibooks dar. Das zweite Kapitel umfasst eine Kapiteleinleitung zur Relevanz von «Metaphern und Vorstellungen» sowie zwölf Wikiseiten, die von Studierenden erstellt und gestaltet wurden. Es wurden acht Metaphern zum thematischen Bereich «Lehren und Lernen ist wie ...» und vier Metaphern zum thematischen Bereich «Bildung ist wie ...» erstellt. Für das dritte Kapitel wurde eine thematische Einführung von mir als Lehrender erstellt. Von den Studierenden wurden drei Wikiseiten zur Darstellung unterschiedlicher didaktischer Modelle, vier Wikibeiträge zur Darstellung von verschiedenen Lerntheorien und vier Wikiseiten mit unterschiedlichen bildungstheoretischen Beiträgen erstellt und gestaltet. Das vierte Kapitel umfasst eine Wikiseite als thematische Einführung zur Relevanz individueller Analysen und Reflexionen der verschiedenen Metaphern. Ferner wurden drei thematisch unterschiedliche Wikiseiten zum Schreiben von Analysen und Reflexionen aus didaktischer, lerntheoretischer sowie bildungstheoretischer Perspektive erstellt. Studierende hatten die Möglichkeit, diese Wikiseiten mitzugestalten. Gemäss der Beschreibung im Kapitel «Deskriptive Analyse der Versionsgeschichte des Wikibooks» (Kap. 3.4.2.2) erfolgte die Analyse der Versionsgeschichte mit der Analysesoftware xTools.

\subsection{Ergebnisse der empirischen Analysen}

Zur wissenschaftlichen Analyse wurden vier unterschiedliche forschungsmethodische Zugänge gewählt (Kap. 3.2.2, 7.3). Dargestellt werden die Ergebnisse der Interviews (Kap. 7.4.1), die Ergebnisse der Veranstaltungsevaluation (Kap. 7.4.2), die Ergebnisse der Metaphernanalyse der studentischen Seminarprodukte (Kap. 7.4.3) sowie die Erkenntnisse aus der Analyse der Versionsgeschichte des Wikibooks (Kap. 7.4.4).

\subsubsection{Ergebnisse der Interviewanalyse}

Die Ergebnisse der Interviewauswertung eröffnen einen systematischen Einblick in die Perspektive der Studierenden. Auf Basis von elf problemzentrierten bzw. episodischen Interviews (Kap. 7.3.1) wird in strukturierender Weise aufgearbeitet, mit welchen Vorkenntnissen und Einstellungen Studierende das Projektseminar belegt und erlebt haben, welche Strategien sie zur Erstellung von Wikiseiten entwickelt haben und wie sie die gesammelten Erfahrungen im Rahmen des Seminars bewerten. So können empirische Indikatoren dafür identifiziert werden, inwiefern das Seminarkonzept geeignet war, um ein Lernen mit und ein Lernen über Medien zu ermöglichen 
(Kap. 3.2). Die Auswertung der durchgeführten Interviews erfolgte in Form einer strukturierenden Inhaltsanalyse (Kap. 3.4.1.1). Zur Darstellung der Ergebnisse wird zwischen vier zentralen Aspekten unterschieden: Einstellungen gegenüber der Projektidee (Kap. 7.4.1.1), Vorkenntnisse und Vorerfahrungen der Studierenden (Kap. 7.4.1.2), Strategien zur Zusammenarbeit und zum Medienhandeln (Kap. 7.4.1.3) sowie Wahrnehmung und Reflexion der (Wikibook-)Gemeinschaft (Kap. 7.4.1.4) ${ }^{210}$.

\subsubsection{Einstellungen gegenüber der Projektidee}

Die Mehrheit der Studierenden war interessiert an der Projektidee und äusserte sich positiv gegenüber dem Vorhaben, im Rahmen eines Seminars gemeinsam ein Wikibook zu erstellen. Die Begründungen für diese Positionierung umfassten verschiedene Elemente. Eines dieser Elemente war die als positive Abwechslung zu anderen Seminaren und Seminarkonzepten erlebte Gestaltung. Diese Abwechslung erläuterten die Studierenden hinsichtlich verschiedener Aspekte, z. B. «Projektarbeit, das ist mal was Anderes» $(B 02,4)$ sowie mit Blick auf «die Zusammenarbeit an sich, das war schon spannend, ja» (B03, 130). Zudem war das Projekt für Studierende «schon noch einmal eine andere Idee von der Art der Veranstaltung, wo man auch aktiver teilnimmt, ja.» (B03, 4). Diese Abwechslung bzw. Abgrenzung zu anderen Seminaren erfolgte hinsichtlich Veranstaltungen, in denen überwiegend Referate und Texte zu vorab vereinbarten Themen diskutiert werden.

«Also, ich fand es mal etwas Anderes, besser als, also ich kenne halt in Pädagogik viele Seminare, wo man hingeht, dann ein Thema zugeteilt bekommt und dann ein Referat halten muss und das war es dann und ansonsten sitzt man blöd rum und macht eigentlich gar nichts. Und ich finde es ganz schön, etwas zu machen, wo man am Ende was in der Hand hat. Also, dann auch weiss, da habe ich mitgemacht oder ich kann auch mal bei anderen reingucken, was haben die gemacht $[\ldots] »(B 09,2)$.

«Also, ich fand es erst mal reizvoll, überhaupt daran teilzunehmen, weil ich halt fand, das ist einmal (kurze Pause) ein anderes Arbeiten als sonst. Also, dass man halt nicht so stupide dasitzt und über Texte diskutiert, sondern dass man halt auch mal was machen kann und einfach mal etwas Praktisches macht» (B11, 2).

Die in den Zitaten positiv hervorgehobene Produktorientierung in Form der praktischen Erstellung eines gemeinsamen Wikibooks wurde auch von weiteren

210 Zur Darstellung der Ergebnisse werden Interviewzitate zur selektiven Plausibilisierung verwendet. Die Referenzierung von direkten Zitaten erfolgt durch die Angabe einer Identifikationsnummer für die befragten Personen sowie die zugehörige Absatznummer im Transkript, z. B. «(B13, 37)». Die verwendeten Namen in den Interviews sind anonymisiert. Die zugehörigen Transkripte befinden sich im Anhang B.6. 
Studierenden markiert. Die Aussagen bezogen sich zum einen auf den eigenen Nutzen, "[a]lso da hat man halt gleich etwas Kompaktes, wo man nachschlagen kann. Das finde ich eigentlich ganz sinnvoll» (B04, 34). Zum anderen wurde der Nutzen für andere beschrieben: «das finde ich eigentlich einen schönen Gedanken, also was produziert zu haben, was jemand anderes nutzen könnte» $(B 10,16)$. Neben dem Interesse am gemeinsamen Produkt in Form eines Wikibooks zum Abschluss des Seminars beschrieben andere Studierende ihr Interesse an den methodischen Möglichkeiten, «Was man so mit welchen Medien da heute macht» $(B 03,130)$ sowie am Prozess der Entstehung eines Wikibooks (B02). In ähnlicher Weise sah eine Studierende, die sich gerne über Wikipedia und Wikibooks informiert, in dem Projekt die Chance einen Einblick «hinter die Kulissen» $(B 01,4)$ zu erhalten:

«Also, das heisst, ich habe als Nutzer selbst schon sehr viel davon profitiert und da ich schon selbst an Wikis mitgearbeitet habe, fand ich es eigentlich auch ganz cool mal selber was zu machen und so ein bisschen hinter die Kulissen, wie viel Arbeit ist das eigentlich, bis das so aussieht, wie man das gerne hätte, weil ich meine, es ist auch schwierig, viel Informationen kompakt darzustellen und trotzdem verständlich zu bleiben. Was ja eines der Ziele ist zum Beispiel» (B01, 4).

Die Frage, welche Bedeutung die Veröffentlichung der produzierten Texte für die Studierenden hat, wurde unterschiedlich beantwortet. Für etwa die Hälfte der Studierenden war das öffentliche Schreiben ein interessantes sowie motivierendes Element (B02, B04, B05, B09, B11). Die Beschreibungen bezogen sich u. a. auf die konzeptionellen Überlegungen zum Seminar. So war eine Person überrascht, «dass halt überhaupt Studenten da was schreiben» (B03, 2), da aus ihrer Erfahrung die Produkte von Studierenden nur für die Leistungserfassung geschrieben und nicht weiter verwendet werden (oder nur in unangemessener Weise) ${ }^{211}$. Das gemeinsame Schreiben eines Wikibooks war für Studierende in diesem Sinne eine interessante Erfahrung, da Gruppen von Studierenden Texte zu einem öffentlichen Wikibook beitragen konnten. Weitere Studierende beschrieben das Wissen darüber, dass ihre Texte von Menschen ausserhalb des Seminars gelesen werden können als Motiv, sich in anderer Weise mit dem Schreiben auseinanderzusetzen. Studierende sagten z. B. «man hatte eine ganz andere Herangehensweise, weil man weiss, ok, das wird irgendwie von Leuten gelesen» (B02, 28), «man hat auch irgendwie ein bisschen einen anderen Anspruch daran.» (B11, 14) und dass «man sich vielleicht mal ein bisschen mehr Mühe [gibt], wenn man weiss: ach das ist jetzt nicht nur für mich» (B09, 2). In diesem Sinne erzeugte die Veröffentlichung für Studierende auch einen gewissen Druck, welcher von einzelnen Studierenden positiv bewertet wurde.

211 Es wurde von einem Fall berichtet, bei dem studentische Arbeitsergebnisse ohne deren Einwilligung in einer Publikation einer Lehrperson verwendet wurden. 
"Also, das hat ein bisschen Druck auf mich ausgeübt, weil ich wusste, dass das, was ich schreibe, irgendwie veröffentlicht wird und jeder darauf Zugriff hat. Aber irgendwo war das auch gut, weil ich das dann ernst genommen habe und nicht so <Larifari) damit umgegangen bin, sondern mich auch mal in die Bibliothek gesetzt habe und Literatur rausgesucht habe. Das hat mir halt auch geholfen, weil ich vorher noch nie etwas verfassen musste und da konnte ich mich halt auch noch damit auseinandersetzen wie man überhaupt Literatur findet und wie man sie anwendet und ja» (B05, 08).

Zudem finden sich Äusserungen von zwei Studierenden, für die die Veröffentlichung keine Bedeutung für die Auseinandersetzung mit den Seminarinhalten und dem Erstellen von Texten hatte. Dies begründeten die Studierenden dahingehend, dass in einem Seminar an der Universität immer ein wissenschaftlicher Anspruch beim Schreiben von Texten verfolgt werden sollte (B01, B10). In den Aussagen von zwei weiteren Studierenden wird sichtbar, dass der durch die Veröffentlichung erzeugte Druck auch problematisiert wurde. So waren sich diese Studierenden unsicher, inwiefern sie einen angemessenen Beitrag zum Wikibook leisten konnten (B06, B08). Die damit verbundenen Zweifel wurden wie folgt beschrieben:

«[...] und was ist jetzt, wenn ich was Falsches schreibe oder etwas total Schreckliches schreibe, was völlig komisch oder falsch ist? Und ich war dadurch halt so ein bisschen eingeschränkt und hatte auch so einen Druck, den ich so empfunden habe, irgendwie so. Also, es war so eine gefährliche Situation irgendwie, also es war nicht so angenehm» (B08, 8).

Ein Studierender äusserte sich skeptisch gegenüber der Notwendigkeit der Projektidee, die gemeinsam erstellten Arbeitsergebnisse im Rahmen des öffentlichen Wikibooks zu schreiben. Diese Person hatte das Gefühl instrumentalisiert zu werden in dem Sinne, dass es mehr darum geht «Wikibooks zu (kurze Pause) voranzubringen und zu perfektionieren anstelle, dass wir Studenten besondere Erkenntnisse erlangen» (B06, 2). Die Auseinandersetzung mit Wikibooks als bis dahin unbekanntes Medium wurde von dieser Person als Nachteil gesehen, weil «ich mich noch mit Formalia auseinandersetzen muss, die mich eigentlich nicht interessieren» (B06, 08). Die Person begründete ihre Perspektive wie folgt:

«Von daher ist es quasi eine zusätzliche Belastung, die mir persönlich, und es geht darum (kurze Pause), natürlich erzeugen wir dadurch irgendwas in der Öffentlichkeit, das andere Leute irgendwie nutzen können, aber ich studiere, ja um mich selbst zu bilden hauptsächlich. Und unter dem Aspekt, habe ich dadurch keinen Mehrwert gesehen» (B06, 10). 
Im Vergleich der elf Interviewten zeigt sich, dass sich die Mehrheit der Studierenden für die Projektidee interessierte und diese aufgrund unterschiedlicher Elemente positiv bewertete. Diese Elemente umfassten (1.) die positive Abwechslung zu anderen Seminaren, (2.) die gemeinsame Entwicklung nützlicher Materialien, (3.) einen Einblick in den Entstehungsprozess eines Wikibooks sowie (4.) die Veröffentlichung der geschriebenen Texte als Motiv für eine aktive Teilhabe. Zugleich wurde von wenigen Studierenden markiert, dass die Veröffentlichung der gemeinsamen Arbeitsergebnisse aufgrund eigener Bedenken als unangenehm empfunden wurde.

\subsubsection{Vorkenntnisse und Vorerfahrungen der Studierenden}

Im Rahmen der Interviews verwiesen die Studierenden bei verschiedenen Antworten auf ihre eigenen Vorkenntnisse und Vorerfahrungen. Diese Vorerfahrungen bezogen sich auf den Umgang mit Wikis und beinhalteten Selbsteinschätzungen des eigenen Kenntnisstandes hinsichtlich der instrumentell-pragmatischen Bedienung eines Wiki-Editors, des Schreibens von Texten sowie thematisch-inhaltlichen Vorkenntnissen. Die Mehrheit der Studierenden kannte die Plattform Wikibooks vor dem Projekt nicht bzw. nutzte diese nicht bewusst (B02, B03, B04, B07). Die Befragten sagten beispielsweise, «[...] dass ich noch nie was von Wikibook erstens mal gehört habe» (B04, 2) oder «[a]lso ich kannte das Vorher gar nicht, also vielleicht hat man schon mal was davon gehört» (B02, 4). Stattdessen ist «Wikipedia [...] da so eher das, was man als Schüler oder Student kennt» (B02, 4). Sieben Studierende nahmen im Gespräch explizit Bezug auf Wikipedia (z. B. B02, B03, B05). Im Fokus steht dabei die rezeptive Nutzung bzw. die Verwendung von Wikipedia als Informationsquelle, wie in der folgenden Aussage exemplarisch zum Ausdruck gebracht wird: «Also, ich benutze das sehr oft, um mich zu informieren, was mir gerade durch den Kopf geht [...]» (B07, 10). Zudem wird in Aussagen zu Wikipedia auch erkennbar, dass Studierende in der Lage sind, Prinzipien von Wikis in beiden Projekten zu erkennen: «Aber, das Grundkonzept, wie es zum Beispiel auch bei Wikipedia ist, finde ich eigentlich gut, dass halt Texte immer wieder überarbeitet werden können» (B10, 18). Interessant ist, dass von sieben interviewten Lehramtsstudierenden nur eine Person deutlich machte, dass sie sowohl mit Wikipedia als auch mit Wikibooks vertraut ist:

«Ok. Ich persönlich finde das eigentlich ganz cool, weil ich selbst gerne mit Wikibooks und Wikipedia arbeite, als Informationsquelle, und es eigentlich immer ganz gut finde, dass man (kurze Pause), wie sagt man, zu den Sachen wo ich dann auch suche, eigentlich immer etwas finde, also gerade Matheschwerpunkt ist Wikipedia recht stark. Und was LaTeX angeht, ist Wikibooks recht stark» (B01, 4). 
In dem Interview wird zudem deutlich, dass die Person nicht nur über Erfahrungen mit Wikis bei der Informationsrecherche verfügte, sondern bereits an Wikis mitgearbeitet hatte. So berichtete die Person von Fachschaftsprojekten, welche mit Wikis organisiert werden. Sie beschrieb in diesem Zusammenhang ihre Kenntnisse über die Wikisyntax als vorteilhaft für die Teilnahme am Wikibookprojekt: «Und vorher kannte ich schon die Syntax schon zum Grossteil und das macht es ja doch ein bisschen leichter» (B01, 6). Weitere Erfahrungen zur Mitgestaltung und Bearbeitung von Wikis wurden von zwei der drei interviewten Pädagogikstudierenden in den Interviews beschrieben. Die Befragten verwiesen dafür auf eine Veranstaltung im Rahmen ihres Studiums, bei denen sie in Tandems an einem DokuWiki arbeiten mussten. Die Veranstaltung wurde von einer Dozentin ${ }^{212}$ des Instituts für Allgemeine Pädagogik und Berufspädagogik angeboten (B08, B11). Die Mitgestaltung von Wikis wurde von einer der zwei Studierenden wie folgt beschrieben:

"Also, erstens hatten wir das ja bei Frau Dunkel vor zwei Semestern, von daher wusste man ja so grob was man damit machen kann. [...] so die grundlegenden Sachen, wie Text hochladen, was ändern und so, dass ist ja eigentlich kein Problem. Dass steht ja auch eigentlich auch da. Bearbeiten (kurze Pause), speichern oder halt Vorschau anzeigen. Ich meine, kann man eigentlich nicht viel falsch machen, von daher fand ich das in Ordnung. Und die Anmeldung war ja auch kein Problem. Wenn man sich schon mal irgendwo anders angemeldet hat im Internet, dann kriegt man das bei Wikibooks glaube ich auch hin» (B11, 30).

Die Mehrheit der Studierenden hatte keine Vorerfahrung mit der Bearbeitung von Wikiseiten sowie hinsichtlich des Umgangs mit der Syntax (z. B. B02, B03, B04). Die Aussagen zu Erfahrungen im Umgang mit einem Wiki-Editor beinhalteten Selbsteinschätzungen der eigenen Kenntnisse, wie beispielsweise «also, ich komme zwar super mit so Computern klar, aber mit so Programmierzeug habe ich echt überhaupt keine Ahnung» (B09, 48). Eine andere Person war dankbar dafür, dass eine Mitstudierende bzw. ein Mitstudierender die Bearbeitung des Wiki-Editors in der Gruppe übernommen hatte, «weil ich mit dieser ganzen Syntax-Geschichte mich komplett neu hätte rein denken müssen und keine Erfahrung mit so etwas habe» (B06, 66). Die verwendete Formulierung der Person «Bin auch sehr dankbar darum [...]» (B06, 66), lässt sich auch als spezifische Haltung im Umgang mit neuen bzw. unbekannten Medien verstehen. Im folgenden Zitat wird exemplarisch deutlich, wie die Person ihre Erfahrungen im Umgang mit dem Wiki-Editor, welche sie u. a. als «Hochladen» bezeichnete, im Zusammenhang mit ihrer distanzierten Einstellung bzw. Haltung gegenüber «Medien» beschreibt:

212 Im Folgenden Frau Dunkel genannt. 
«Befragte Person: Ja da bin ich ehrlich gesagt nicht so der Fan mit Medien. Ich bin da eher ein bisschen Oldschool.

Interviewer: Ok. Wenn du Oldschool sagst, wie meinst du das?

Befragte Person: Ja, also ich benutze schon Word und benutze da ein paar Sachen. Aber dieses Hochladen und irgendwas, irgendwelche Kommentare anschauen, das ist nichts für mich. Ich drucke das dann lieber aus, gebe das dann jemandem, der liest sich das dann durch und deswegen ich habe auch noch nie etwas hochgeladen. Ich war schon überfordert, als ich diese E-Mail in der Gruppe schreiben sollte in Moodle und eine Menge Leute anschreiben sollte $[\ldots . .].(B 05,88-90)$.

Neben den thematisierten Vorerfahrungen mit öffentlichen Wikis sowie bezüglich des Umgangs mit dem Wiki-Editor beschrieben Studierende weitere Aspekte. Beispielsweise äusserte sich eine Person distanziert gegenüber den verschiedenen Arbeitsaufträgen in studentischen Arbeitsgruppen aufgrund eigener Vorerfahrungen: «Da muss ich halt ehrlich sagen: Ja, ich will Lehrer werden, aber ich bin kein Fan von Gruppenarbeiten, weil ich damit bisher noch nie gute Erfahrungen gemacht habe» (B04, 04). In weiteren Aussagen wurden von Studierenden (fehlende) Erfahrungen mit wissenschaftlichen Schreibstrategien aufgrund der geringen Fachsemesteranzahl thematisiert, «weil, wenn man neu an der Uni ist und dann im zweiten Semester so ein Projekt hat, das ist halt schon etwas Anderes» (B02, 30). Entsprechende Äusserungen wurden allerdings nicht von Studierenden in höheren Fachsemestern getroffen. Wie bereits im vorherigen Kapitel skizziert, wurden von den wenigen Studierenden auch inhaltliche Vorerfahrungen beschrieben. Die verschiedenen Vorerfahrungen der Studierenden können als Indikator für die Heterogenität der Befragten hinsichtlich der genannten Aspekte gedeutet werden.

Vergleicht man die Aussagen der elf Interviews ist auffällig, dass die Mehrheit der Studierenden Wikipedia als öffentliches Wiki kannte und als Informationsquelle verwendet. Allerdings verfügen nur drei Studierende über Erfahrungen hinsichtlich der Mitgestaltung öffentlicher Wikis und lediglich eine Studierende kannte das Wikibook als Schwesterprojekt der Wikipedia.

\subsubsection{Strategien zur Zusammenarbeit und zum Medienhandeln}

In den Interviews wurden Studierende explizit zur ihrem «Vorgehen zum Schreiben der Wikibeiträge» (Anhang A.1.1) befragt. Zur strukturierten Darstellung der Analyse dieser Aussagen werden im Folgenden drei Aspekte unterschieden (a) Schreiben (und Lernen) in Gruppen, (b) Gruppenkommunikation und -organisation via Moodle und WhatsApp sowie (c) Strategien und Reflexionen zum (gemeinsamen) Schreiben mit Wikis. 


\subsection{Schreiben (und Lernen) in Gruppen}

Über die Hälfte der Befragten (B2, B6, B8, B9, B11) gaben im Interview einen Einblick in ihr Vorgehen während der ersten Seminarphase «Vorstellungen sichtbar «machen » (Kap. 7.2.2) und beschrieben das Erleben der ersten Gruppenarbeitsphase (B2, B4, B5, B6, B10, B11). In diesen Beschreibungen finden sich verschiedene Hinweise dafür, dass verschiedene Studierende die Texte in weitgehend kollaborativer Zusammenarbeit erstellt haben. So verwiesen zwei Studierende auf den ersten Seminartag, an dem sie bereits mit der Erstellung des Textes begonnen hatten (B8, B9). Ihren kollaborativen Austausch beschrieb eine Person in der Form, dass sie einander ein Textdokument "geschickt» und bearbeitet hatten (B9). Eine weitere Person beschrieb die gelungene Zusammenarbeit in der ersten Phase dadurch, dass sie sich am Seminartag «total gut absprechen» konnte (B8). Von zwei weiteren Studierenden wurde die Bedeutung des inhaltlichen Austauschs bei gemeinsamen Treffen zur Texterstellung hervorgehoben (B2, B11):

«Ja. Also in der ersten Gruppe, bei der Metapher, haben wir das komplett zusammen geschrieben, also ich habe zum Beispiel getippt und der Rest war halt irgendwie dabei. Es war zwar ein bisschen schwierig auf einem Rechner da zu sechst drumrum zu sitzen, aber es war auf jeden Fall sehr gut, weil jeder sich eingebracht hat und da super schnell ein Text zu Stande gekommen ist und jeder auch alle so in einen einheitlichen Konsens hatten» $(B 11,26)$.

Im Gegensatz dazu wurde von einer Person ein anderes Vorgehen beschrieben, welches kaum Elemente zum Austausch aufweist. So arbeitete diese studentische Arbeitsgruppe in der Phase «Diskussion und Produktion» (Kap. 7.2.2.1) des ersten Blocktermins arbeitsteilig: «Wir haben uns zweigeteilt. Klara und ich haben uns um den Text gekümmert. Und die anderen beiden haben sich um den Videobeitrag gekümmert» (B06, 59). Das «gemeinsame Kümmern» um den Text erfolgte gemäss der Beschreibung jedoch weitgehend ohne die Beteiligung dieser Person, die wie sie selbst sagt, «geringe bis keine Motivation» $(\mathrm{B} 06,61)$ hatte:

«War auch wiederum schwierig, weil Klara halt den Text geschrieben hat. Ich sass halt daneben daran und (habe) mir gedacht: Na gut, das würde ich jetzt anders formulieren, das würde ich jetzt anders formulieren, das (unverständlich). Ich hätte es halt ganz anders geschrieben» (B06, 59).

Der Umgang mit den Rückmeldungen zu den erstellten Texten in der ersten Seminarphase (Kap. 7.2.2.2) wurde von drei Studierenden beschrieben (B01, B04, B06). Es wird zum einen deutlich, dass sich die zwei Studierenden mit den Rückmeldungen auseinandergesetzt haben: «Ich habe mir die Kritik angeguckt, habe mir so gedacht: Oh ja Scheisse, ja, ja, ja und das muss man alles noch verbessern» $(B 06,57)$, sowie, 
"Gut, also ich sage jetzt einmal, kam ja jetzt was zu den Metaphern, dass wir die noch einmal überarbeiten sollten» (B04, 18). Zum anderen bringen die Studierenden zum Ausdruck, dass die Überarbeitung der Metaphern zum Zeitpunkt des Interviews noch nicht erfolgt war. Dies begründeten die Studierenden in einem unterschiedlichen Grad der Distanzierung damit, dass in ihren Gruppen nicht klar war, wer sich darum kümmern sollte, wie sich an folgenden Formulierungen zeigt «Im Endeffekt hat sich niemand zuständig gefühlt» (B06, 57), und, «[...] aber ich weiss halt, also ich habe halt wirklich das Gefühl, wenn ich es jetzt nicht mache, wird es nicht gemacht» (B04, 18). Dieses Phänomen beschrieb eine weitere Person als «Verantwortungsdiffusion» $(B 10,4)$ innerhalb der Gruppe, welches sich auch in weiteren Arbeitsphasen zeigte. Für eine Person war damit eine grundsätzliche Problematik des Umgangs mit dem Wikibook verbunden.

«Also immer die Deadline ist da, ich muss es mal reinstellen, aber danach muss ich es mir auch nicht noch einmal angucken oder so. Also sieht man ja die erste Phase, da wurde ja glaube ich gar nichts - soweit ich es gesehen habe - beim letzten Stand überarbeitet» (D01, 8).

Die Zusammenarbeit in der zweiten Seminarphase zur «Erarbeitung erziehungswissenschaftlicher Denkwerkzeuge» (Kap. 7.2.3) wurde in allen Interviews beschrieben. Dabei lassen sich im Sinne von Hinze (2008) kollaborative, kooperative sowie kollektive Formen der Zusammenarbeit identifizieren (Kap. 6.4). Studentische Vorgehensweisen mit mehreren Austausch- und Kommunikationsphasen sowie einem gemeinsamen Schreiben am Text bzw. dem Durchlaufen unterschiedlicher Überarbeitungsschritte werden als kollaborative Form der Zusammenarbeit verstanden. Zwei Studierende beschrieben eine Form der kollaborativen Zusammenarbeit dahingehend, dass sie damit begannen individuell zu lesen, um sich anschliessend in der Gruppe über die Texte auszutauschen (B04, B09). Dies beschrieb eine Person beispielsweise wie folgt: «Wir haben uns jeder zuerst einmal schlau gelesen über die Themen und haben uns, bevor wir geschrieben haben, einmal getroffen» $(B 4,10)$. Nach dem Austausch über die gesichteten Texte einigten sich die Studierenden in den zwei Gruppen jeweils darauf, sich zum Schreiben bestimmter Abschnitte aufzuteilen: «Also wir haben dann gesagt: Okay du schreibst jetzt eher dann hier darüber und jemand anderes schreibt dann über Kritik [...]» $(B 09,10)$.

«Dann haben wir uns entschieden, wer über was schreibt. Also wir haben es sozusagen aufgeteilt, dass jeder etwas schreibt (kurze Pause) und haben dann eben beschlossen, dass wir uns das eben in Moodle hochladen und eben uns gegenseitig korrigieren. Das hat auch soweit ganz gut geklappt» (B04, 10). 
Zum Zusammenstellen des finalen Textes erfolgten, wie im Zitat deutlich wird, gegenseitige Korrekturen sowie weitere Treffen zum Austausch. Von weiteren Studierenden wurden ähnliche Formen der kollaborativen Zusammenarbeit beschrieben, welche zunächst durch die Aktivität weniger Gruppenmitglieder initiiert wurden (B01, B03, B04, B05, B06). Eine bzw. zwei Personen hatten damit begonnen einen ersten Textentwurf zu erstellen, welcher daraufhin mit weiteren Gruppenmitgliedern besprochen und bzw. oder von diesen ergänzt und überarbeitet wurde. Wie sich in den folgenden Aussagen zeigt, war der Grad an Austauschprozessen hier geringer als bei den zwei zuvor skizzierten Beispielen:

"[...] hat sie es übernommen, quasi die (kurze Pause) den ersten Entwurf zu machen, den wir auch besprochen haben. Und dann war ja die Sache, ja gut, sie hat dann gemeint: Von wegen, ich habe jetzt den ganzen Kram gelesen und habe jetzt hier Entwurf gemacht, schreib du den Text. Ja gut, dann schreibe ich halt den Text. Habe mir halt die Literatur zur Hand genommen und habe versucht, halt einen Text zu formulieren. Habe inn dann noch einmal als Rücksprache, also noch einmal geschickt $[\ldots]$ (..] (B06, 61).

«Aber dann haben wir das, ne, eine von uns hat Stichpunkte schon mal geschrieben, hat die reingestellt und dann hat jeder ergänzt. Jeder hat mal geschaut und irgendwie hat sich das wer aufgeteilt. Bei der Ausarbeitung haben dann die einen die analytische Dimension, die anderen die normative, und so haben wir das dann geschrieben. Der eine hat eine Literaturliste gemacht, der andere die Einleitung» (B05, 34).

Vier Studierende beschrieben die Gruppenstrategie zum Schreiben eines Wikibeitrages überwiegend als arbeitsteiliges Vorgehen, bei dem zum Abschluss die einzelnen Textteile zu einem Text zusammengetragen wurden (B02, B08, B10, B11). In Anlehnung an Hinze (2008) werden entsprechende Beschreibungen als kooperatives Vorgehen verstanden. In Abgrenzung zu den zuvor beschriebenen Strategien wurden von den Studierenden bis zum Zusammentragen der Einzelergebnisse keine Diskussionsprozesse oder Überarbeitungsschleifen benannt. Stattdessen erscheinen die beschriebenen Prozesse jeweils drei Elemente zu beinhalten: (1.) die Aufteilung der Aufgaben, (2.) die eigenständige Bearbeitung sowie (3.) das Zusammentragen der Ergebnisse. Die ersten zwei Elemente werden exemplarisch wie folgt beschrieben: «Also, wir haben am Anfang diesen Text, den du uns geschickt hast, diesen aus dem Reclam-Heftchen, erst haben wir das so nach Sinnabschnitten geteilt, jeder hat seinen Text geschrieben» (B02, 10). Die eigenständige Bearbeitung und das Zusammenstellen der Texte wurden in einem anderen Interview wie folgt beschrieben: 
«Eigentlich haben wir alles so relativ in Eigenarbeit gemacht. Erst haben wir uns über das Thema informiert, jeder hat etwas mitgebracht, dann haben wir uns zusammengesetzt [...]. Genau, und dann haben wir das halt eben zusammengetragen und so ein bisschen ergänzt» (B08, 23-25).

Wenn die eigenständige Bearbeitung der Teiltexte nicht (angemessen) erfolgte, wurde dieses Vorgehen von verschiedenen Studierenden problematisiert. Dies zeigt das folgende Zitat: «[...] zum Beispiel gerade dieses, die Lerntheorien, die wir erarbeitet haben, das steht bei uns immer noch nicht richtig, weil man dann wartet darauf, auf die Arbeit von den anderen [...]» (B10, 4). Zugleich finden sich Beschreibungen, bei denen Studierende ihre Strategien im Verlauf geändert hatten. In einem Interview wurde davon berichtet, dass auf das Bestreben zweier Studierender die Gruppe zunächst eine kooperative Strategie verfolgte: «Wir machen das jetzt so, dass jeder einen Teil schreibt und wir basteln das zusammen» (B11, 26). Im Verlauf der Gruppenarbeitsphase entstanden jedoch Probleme, woraufhin sich ein Teil der Gruppe dazu entschied, sich häufiger zu treffen und eher kollaborativ vorzugehen.

«Vor allem haben wir dann festgestellt, dass diese Struktur die wir erst hatten auch noch völliger Schwachsinn war. Und wir das dann im Endeffekt in zweimal Treffen, wo diese beiden Leute die das vorgeschlagen haben nicht einmal da waren, dann irgendwie wieder zusammen gewurschtelt haben und natürlich auch die Blöcke in unterschiedlichen Dimensionen reingekommen sind (kurze Pause) und wir uns dann zu zweit, zu dritt noch einmal hingesetzt haben um das noch einmal komplett zu lesen, dass es wenigstens einigermassen einheitlich klingt, dass der Sprachstil wenigstens einheitlich ist» $(B 11,26)$.

In einem Interview wurde davon berichtet, dass sich die Gruppe zunächst ebenfalls den zu schreibenden Text aufteilte, jedoch kein Austausch oder Treffen zum Zusammentragen des Textes erfolgte (B07). Aufgrund von Problemen bei der Gruppenorganisation veröffentlichten die Studierenden jeweils einzeln ihre Texte im Wikibook. Diese Form der «Zusammenarbeit» kann eher als kollektive Form der Zusammenarbeit beschrieben werden, da eine gegenseitige Überarbeitung ausblieb.

Der Umgang mit den Rückmeldungen zur zweiten Seminarphase wurde von drei Studierenden ausführlicher beschrieben (B05, B06, B08). Es wurde positiv markiert (B06), dass die Möglichkeit bestand, sich im Seminar über die Online-Rückmeldungen auszutauschen. Zum Umgang mit den Rückmeldungen beschrieben zwei Studierende ein arbeitsteiliges Vorgehen in dem Sinne, dass sich die Studierenden die Überarbeitung der Kritikpunkte aufteilten und einzeln bearbeiten wollten. In welchem Umfang bei diesem Vorgehen Austausch- und Kommunikationsprozesse erfolgten, blieb offen, wie das folgende Zitat exemplarisch belegt: 
"Und jetzt auch beim Korrigieren, da war ja das Feedback, dass wir unsere Quellen, also, dass wir die auch noch zitieren sollen im Text, drin. Da haben wir das auch aufgeteilt, dass zwei sich an der normativen Ebene und zwei an der analytischen orientieren» $(\mathrm{B} 05,34)$.

Beim Vergleich der Interviews zeigen sich in der ersten Seminarphase kollaborative und arbeitsteilige Vorgehensweisen zum Erstellen der Texte durch die studentischen Arbeitsgruppen. Die kollaborative Zusammenarbeit wurde von den Studierenden jedoch nicht mithilfe des Wiki-Editors beschrieben, sondern u. a. durch das Versenden von Dateien realisiert. In der zweiten Seminarphase beschrieben die Studierenden kollaborative, kooperative und kollektive Vorgehensweisen zum Schreiben der Textprodukte. Kollaborative Arbeitsformen im Sinne einer gegenseitigen Korrektur und Überarbeitung von Texten wurden von sechs Studierenden beschrieben. Diese Form wurde häufig von einer Person initiiert. Weitere vier Studierende beschrieben kooperative bzw. arbeitsteilige Vorgehensweisen. Die Rückmeldungen wurden von den Studierenden als nachvollziehbar beschrieben, führten jedoch in der ersten Phase u. a. aufgrund einer «Verantwortungsdiffusion» nicht zur Überarbeitung.

\subsection{Gruppenkommunikation und -organisation via Moodle und WhatsApp}

Mit der Verwendung von Moodle wurde u. a. das Ziel verfolgt, die Gruppenarbeit der Studierenden zu unterstützen (Kap. 6.6.2). In den Interviews mit Studierenden wurde die Verwendung von Moodle für unterschiedliche Ziele beschrieben und unterschiedlich bewertet von (B01, B02, B03, B04, B05, B08, B10, B11). Zwei Studierende berichteten davon, dass sie ihre Ergebnisse über Moodle hochgeladen hatten (B08, B11). Die Sichtbarkeit der Ergebnisse über Moodle eröffnete für eine Studierende die Möglichkeit, sich über Fortschritte der anderen studentischen Kleingruppen zu informieren und Rückfragen zu stellen.

«Ich habe im Prinzip immer, wenn ich gesehen habe, es ist etwas Neues da, habe ich es gelesen. Weil, ich war ja in allen Sitzungen da, ich weiss ja, was inhaltlich in den Sitzungen passiert ist, theoretisch zumindest, wie genau man das noch weiss ist ja die Frage. Dann habe ich geguckt, was daraus geworden ist und habe dann (kurze Pause) immer komplett gelesen und hab dann meistens sowas geschrieben wie: Ja, inhaltlich kann ich alles nachvollziehen, ich weiss natürlich nicht, ob es vollständig ist, weil ich mich nicht mit dem Thema auskenne, und habe dann halt formale oder inhaltliche Fragen gestellt» (B01, 38). 
Die Verwendung von Moodle zum Austausch innerhalb der studentischen Arbeitsgruppe wurde von fünf Studierenden beschrieben (B02, B03, B04, B05, B10). In drei Interviews wurde die Verwendung von Moodle als positiv und praktisch im Vergleich zu E-Mails charakterisiert: «[...] ich finde Moodle besser, wenn man wirklich Dokumente austauscht» $(\mathrm{B} 03,50)$, «dass wir uns das eben in Moodle hochladen und eben uns gegenseitig korrigieren. Das hat auch soweit ganz gut geklappt» (B04, 10), sowie: "obwohl ich mittlerweile eigentlich die Moodle-Kommunikation eigentlich ganz gut finde, also dieses chatten quasi, ähm, weil man dann auch alle Teilnehmer direkt in dieser Gruppe ja eigentlich drin haben sollte» (B10, 6).

Von einer Person wurde markiert, dass die benannten Vorteile abhängig von der Gruppe sind: «Ich glaube bei manchen Gruppen klappt es besser, bei manchen weniger. Bei meiner zweiten, der Lerntheorie, hat es eher schlecht geklappt, [...]» $(B 10,6)$. Das Scheitern von Austauschprozessen wurde von drei weiteren Studierenden im Zusammenhang mit Moodle beschrieben. So problematisierten die Befragten, dass die verschickten Nachrichten über Moodle von ihren Gruppenmitgliedern nicht gelesen wurden: «nur irgendwann kam dann halt überhaupt nichts in Moodle und irgendwie es kam auch keine Kommunikation zustande» $(\mathrm{B} 04,10)$, sowie «[...] das ging irgendwie so ein bisschen, naja nach hinten los will ich nicht sagen, aber dann: Oh ich war nicht bei Moodle online, ich habe die Nachricht von euch nicht gesehen. Und dann dachte man sich: Ja super» (B02, 32). Die Problematik mit der ausbleibenden Kommunikation über Moodle führte bei den Studierenden dazu, dass sie sich im Prozess der Gruppenarbeit bzw. in folgenden Gruppen darum bemühten, alternative Kommunikations- und Austauschmöglichkeiten zu nutzen.

«Weil über Moodle da hat man dann was geschrieben und es kam einfach keine Antwort zurück. Daraus haben wir gelernt und dann haben wir bei der zweiten Gruppe Nummern ausgetauscht und da lief es halt prima, da konnte man auch ständig schreiben und da haben wir auch eine Dropbox, einen Ordner, eröffnet und da konnte jeder die Sachen hochladen, bearbeiten und so sind wir dann im Endeffekt dazu gekommen. Also, jeder hat mal darüber gelesen und eigene Sachen mit reingeschrieben» $(B 05,28)$.

Der Austausch von Telefonnummern, wie in dem Zitat beschrieben, diente den Studierenden zum Erstellen von WhatsApp ${ }^{213}$-Gruppen. So beschrieben sieben Studierende die Verwendung von WhatsApp zum Austausch innerhalb der Gruppen (B02, B03, B04, B05, B07, B08, B11). Der Austausch über WhatsApp umfasste inhaltliche sowie organisatorische Fragen, z. B. zur Vereinbarung von Treffen, zum Austausch von Literatur, zur Krankmeldung innerhalb der Gruppe bzw. zur Kommentierung von

213 Die App «WhatsApp» kann als Instant-Messenger-Anwendung bezeichnet bzw. kategorisiert werden. Auffällig war im Rahmen der Analyse, dass die befragten Studierende nur die Nutzung von WhatsApp erwähnten. Die Verwendung alternativer Instant-Messenger-Anwendung wurden Seminarkontext nicht beschrieben. 
Textentwürfen. Für die Befragten, die sich für den Austausch über WhatsApp entschieden hatten, schien die Verwendung von WhatsApp nahezu selbstverständlich. Die Person, welche sich als «nicht so der Fan mit Medien» und als «eher ein bisschen Oldschool» beschrieb $(B 05,90)$, charakterisierte die Vorteile von WhatsApp beispielsweise wie folgt:

"Ja das Gute bei WhatsApp ist halt, da kommt man nicht drumrum, da muss man antworten, weil man ja sieht, wer was wann gelesen hat. Und mit einer EMail ist das ja nicht so, da verschickt man eine E-Mail und wenn man keine Lust hat, das hochzuladen, dann liest man die einfach nicht. Das war das Problem mit der ersten Gruppe zum Beispiel, dass man nicht einmal darauf eingegangen ist» $(B 05,40)$.

Es zeigte sich jedoch im Gespräch mit einer weiteren Person, dass auch das Vorhandensein einer WhatsApp-Gruppe keine Garantie für einen Gruppenaustausch sowie eine gelungene Gruppenkommunikation ist. So hat für diese Person, die zweite Gruppenarbeitsphase «nicht so ganz toll funktioniert» (B07, 12). Zwar hatten die Arbeitsgruppe eine WhatsApp-Gruppe erstellt aber «da hat kaum einer was geschrieben» $(B 07,12)$.

Beim Vergleich aller Interviews zeigt sich, dass über die Hälfte der Studierenden nahezu selbstverständlich über WhatsApp kommunizieren. Die Möglichkeiten zur Gruppenkommunikation über Moodle nutzten nur wenige Studierende.

\subsection{Strategien und Reflexionen zum (gemeinsamen) Schreiben mit Wikis}

Mit dem Projekt wurde u. a. das Ziel verfolgt, über das gemeinsame Schreiben eines freien und öffentlichen Fachbuchs, einen Beitrag für eine offene und partizipative Kultur zu leisten (Kap. 4.5.2.1). In Form dieser handlungsorientierten Auseinandersetzung wurde das Potenzial gesehen, Lernhandlungen zur Entwicklung der Medienkompetenz der Studierenden anzuregen (Kap. 6.4.2). Eine entsprechende Entwicklung erschien $u$. a. in den Bereichen Gestalten und Veröffentlichen eigener Wikibeiträge (Kap. 6.4.2.1) sowie Auswahl und Nutzen von sozialen Medien zur Kooperation und zum Lernen (Kap. 6.4.2.3) möglich. In der Analyse der Interviews zeigten sich diesbezüglich unterschiedliche Handlungsstrategien und Reflexionen zum (gemeinsamen) Schreiben mit Wikis.

Zunächst ist auffällig, dass die Studierenden zum gemeinsamen Schreiben ihrer Texte für das Wikibook zunächst nicht den Editor von Wikibooks verwendeten. Die Verschriftlichung der studentischen Beiträge erfolgte bei allen befragten Studierenden zunächst über lokal gespeicherte Dateien. Die Mehrheit der Interviewten sprach in diesem Zusammenhang von «Word» (B03, B05, B07), «Worddateien» (B08, B11) und «Worddokumenten» (B04, B09) als zentrales Dateiformat bzw. als Programm zur 
Textverarbeitung. Drei Studierende sprachen in ihren Interviews neutral von Texten (B02, B06, B11) und eine weitere Person verwendete LaTeX zum Schreiben ihrer Textbeiträge (B01). Fast alle Befragten beschrieben ihre Vorgehensweise zum gemeinsamen Schreiben in der Form, dass sie lokal gespeicherte Dateien über die eingerichteten Moodle-Foren (B04, B10), über E-Mails (B03, B04, B06, B11) oder über einen Clouddienst (B05) miteinander austauschten. Die folgenden zwei Zitate veranschaulichen diese studentischen Vorgehensweisen exemplarisch:

«Genau, also wir hatten so einen Ordner noch in Neurowissenschaften und dann hab ich eben eingeladen und dann konnte jeder seine Worddatei hochladen. Wir hatten eine Datei und da hat jeder irgendwas geschrieben und verbessert. Das ist halt auch gut, wenn jeder die Möglichkeit hat, mal darauf zuzugreifen und da hat jeder mal einen Rechtschreibfehler korrigiert» (B05, 30).

«Dann haben wir wirklich die Word-Datei immer rumgeschickt und immer aktualisiert, was total anstrengend war, weil es mit den Treffen einfach nicht funktioniert hat. Dann haben wir das teilweise auf Moodle hochgeladen. Zum Schluss war es uns, wie gesagt dann einfach langsam echt egal, dann haben wir es einfach auf Wikibook hochgeladen» $(B 11,30)$.

Die Formulierung des «Hochladens» im zweiten Zitat ist eine interessante Sprachfigur der Studierenden. Im Zusammenhang mit dem Erstellen einer Wikiseite und dem Schreiben eines Textes mithilfe des Wiki-Editors verwendeten - bis auf eine Ausnahme - alle Befragten den Begriff «Hochladen» ${ }^{214}$, z. B. «Und beim zweiten Mal haben wir uns das halt aufgeteilt, haben es dann in ein Worddokument gemacht, haben das dann bei Wiki hochgeladen» $(B 09,16)$ oder «Da kann ich jetzt gar nichts dazu sagen, da hat die Franziska gesagt, sie lädt das hoch» (B03, 70). Das Interessante an der Formulierung ist zum einen, dass der Begriff «Hochladen» im Fachdiskurs nicht für die Bedienung des kollaborativen Texteditors verwendet wird (Kap. 4.3.3), sondern vielmehr für den Umgang mit Dateien.

Das Schreiben bzw. Veröffentlichen des Textes mithilfe des Wiki-Editors wurde in diesem Zusammenhang von den Befragten in drei Formen beschrieben: (1.) als «Hochladen» durch eine «Technikbeauftragte» bzw. einen "Technikbeauftragten», (2.) als zu klärende «Verantwortungsdiffusion» vor der Abgabe sowie (3.) als Auseinandersetzung mit der Wikisyntax und Orientierung an vorhandenen Beiträgen. Mit der ersten Formulierung, des «Hochladens» durch eine «Technikbeauftragte» bzw. einen «Technikbeauftragten», wird eine Gruppensituation beschrieben, in der sich jeweils eine Person bereit erklärt, die Veröffentlichung des vorgeschriebenen Textes

214 In den Interviews mit B03, B04 und B06 wurde der Begriff «Hochladen» auch vom Interviewer verwendet, nachdem die Studierenden diesen Begriff im Rahmen der Interviews zum ersten Mal geäussert hatten. Im Interview mit B05 wurde den Begriff zum ersten Mal vom Interviewer verwendet. Zuvor hatte die Person den Begriff «Hochladen» im Zusammenhang mit einem Clouddienst verwendet. 
über Wikibooks zu übernehmen (B02, B03, B04, B05, B06, B11). In den Erzählungen der Studierenden erfüllten die entsprechenden technikbeauftragten Personen diese Aufgabe weitgehend freiwillig, u. a. weil diese Vorerfahrungen bezüglich des Schreibens von Wikibeiträgen hatten. Entsprechende Formulierungen waren z. B. «Sie hat gleich von Vornherein gesagt, sie ist da fit darin. Für sie ist das eine Sache von zwei Minuten so ungefähr, dass da reinzustellen mit den Formalia» (B06, 66), «Ja, in den anderen Gruppen war immer einer, der gesagt hat: Ja ich mach das, ich habe da voll den Durchblick, ich kann das, ich habe das jetzt schon öfters gemacht» (B04, 28), sowie «Also wir haben dann unseren Technikfachmann beauftragt, das doch bitte alles bei Wikibooks hochzuladen, das war dann aber auch ok» (B02, 36). Die Bearbeitung durch die «Technikfachfrau» bzw. den «Technikfachmann» beschrieben die Befragten mehrheitlich als dankbare Situation, da sich sie nicht mit der Wikisyntax auseinandersetzen mussten. Die folgenden Zitate veranschaulichen diese Position exemplarisch: «Bin auch sehr dankbar darum, weil ich mit dieser ganzen Syntax-Geschichte mich komplett neu hätte reindenken müssen und keine Erfahrung mit so etwas habe» (B06, 66), und «[...] dafür war, wie gesagt, der Ben zuständig. Der hat gleich gesagt, er macht das, das ist überhaupt kein Ding und da (kurze Pause) bin ich dann auch froh $[\ldots] »(B 11,30)$.

Die zweite Formulierung, die zu klärende «Verantwortungsdiffusion» (B10, 4), beschreibt eine Gruppensituation, in der bis zum Abgabedatum eines Textes ungeklärt war, wer das Schreiben des Wikibookbeitrages übernimmt (B04, B05, B08, B10). Die von einer Person geäusserte Metapher der «Verantwortungsdiffusion» kann so verstanden werden, dass es in der Gruppe nicht klar (bzw. diffus) war, wer die Verantwortung für das Veröffentlichen des Wikibeitrages übernehmen sollte: «und gerade dadurch, dass man das beim Wiki hochlädt, ist so eine gewisse Verantwortungsdiffusion, also irgendwer wird es ja schon machen, irgendwer wird es schon hochladen, glaube ich» (B10, 4). Ähnliche Situationen wurden auch von weiteren Studierenden beschrieben:

«Also, es war so, ja bei uns in der Gruppe hat das mit der Kommunikation irgendwie nicht richtig funktioniert, also es war ein bisschen komisch. Erst hiess es, jeder guckt mal, ob er es reinstellen kann und (kurze Pause) dabei sind wir geblieben. Dann hat jeder geguckt, ob er es reinstellen kann (Lachen), und es war halt nichts drinnen dann auch am Abgabetermin. Also wir hatten es dann auf Moodle, aber halt nicht in dem Wikibook» (B08, 38).

Die dritte Strategie zur Erstellung von Wikiseiten umfasst eine Auseinandersetzung mit der Wikisyntax sowie eine Orientierung an vorhanden Beiträgen. Dieses Vorgehen beschrieben Studierende, die sich dieser Auseinandersetzung alleine oder mit mehreren Gruppenmitgliedern gemeinsam stellten (B01, B07, B09) sowie Studierende, die aus der Situation der Verantwortungsdiffusion heraus sich dazu entschieden, 
die Erstellung der Wikiseiten zu übernehmen (B04, B05, B08, B10). Diese Entscheidungen wurden zum Teil als einzige Option beschrieben, «weil es keiner machen wollte» (B04, 24) bzw. als Abwägungsprozess dargestellt:

«Ja, da waren da, also, da war es den einen wichtiger, das hochzuladen, den anderen nicht, und denen es wichtiger war, die haben dann gesagt: Ja wir müssen das jetzt hochladen, weil. Okay das ist dann blöd, weil die anderen mitgewinnen sozusagen und so dastehen, als hätten die das auch hochgeladen, aber in dem Moment war das egal, weil wir machen das ja auch für uns und wenn man immer daran denkt, was andere machen und was sie nicht machen, dann kommt man ja nie voran» (B05, 42).

Die Nutzung des Wiki-Editors zur Erstellung einer Seite sowie zur Formatierung des vorliegenden Textes wurde von den Studierenden in unterschiedlicher Weise dargestellt. Vier Studierende beschrieben, dass sie das Erstellen und Bearbeiten einer Wikiseite mit mehreren Gruppenmitgliedern durchführten (B05, B07, B08, B09). Andere Studierende übernahmen die Bearbeitung der Wikiseiten alleine (B01, B04, B10). Hinsichtlich des konkreten Vorgehens wiesen zwei Studierende ohne Vorerfahrungen darauf hin, dass sie sich zur Formatierung ihres eigenen Textes an bereits erstellten Wikibeiträgen orientierten (B07, B09). Dies beschrieb eine Studierende wie folgt: «Wir haben immer geguckt wie es die anderen davor gemacht haben. Sind dann da halt auch auf Bearbeiten gegangen, haben halt geguckt welche Zeichen die halt davor gemacht haben oder so» (B09, 18). Die Einschätzung der Schwierigkeit dieser Aufgabe, wurde von zwei weiteren Studierenden ohne Erfahrungen im Umgang mit Wikibooks sehr unterschiedlich bewertet. Eine Person (B10), die in beiden Phasen die Erstellung der Wikiseiten übernommen hatte, fand es «jetzt auch nicht so schlimm, dass jetzt mal kurz da einzustellen» (B10, 13). Da der Text schon fertig geschrieben war, «man muss es nur noch hochladen» und aus ihrer Sicht ist das «relativ einfach irgendwie» (B10, 12). Eine andere Person (B04) kam nach ihrer Auseinandersetzung mit dem Wikibook, weil es in ihrer Gruppe niemand sonst machen wollte, zu einer deutlich anderen Einschätzung:

«Ja, also ich habe fast eine Stunde gebraucht, um es in Wikibook einzustellen und mir war es dann, wenn ich ehrlich bin, egal, ob es richtig ist oder nicht, ich war einfach froh, dass es dann drinnen ist und dachte nur: das kann man ja noch verändern und verbessern» $(\mathrm{B} 04,54)$.

«Also, ich glaube, wenn ich es jetzt noch einmal machen würde, wüsste ich es besser, wie es geht, oder, es war halt das erste Mal und dann war es halt für mich, als nicht medialer Mensch, relativ schwierig» (B04, 26). 
Für diese Person, die sich selbst als «medial nicht auf der Höhe» (B04, 18) bezeichnet, war die Erstellung eines Wikibeitrages eine echte Schwierigkeit. Zugleich beinhalteten ihre Beschreibungen der Situationen bereits Reflexionen über ihre Mitgestaltungsmöglichkeiten an öffentlichen Wikis. Die Hinweise auf die Veränderbarkeit ihres erstellten Textes lässt erkennen, dass sich die Person den Prinzipien der Prozessualität und Partizpation bewusst ist (Kap. 6.4.2.2). Ferner schätzte sie ihre eigene Fähigkeit zum zukünftigen Umgang mit dem Wiki-Editor als «besser ein». Auch weitere Studierende brachten in ihren Beschreibungen zum Ausdruck, dass sie durch die Auseinandersetzung mit dem Wiki-Editor etwas über die Gestaltung von Wikiseiten mithilfe der entsprechenden Syntax gelernt haben (B01, B07, B08). Ferner beschrieben Studierende, dass ihnen durch die Erstellung eigener Beiträge die Angst bzw. der Respekt vor der Aufgabe genommen wurde (B08, B09).

«Also anfangs war das darauf bezogen, dass ich eben (kurze Pause) also komisch fand, diese Übertragung und mir das gar nicht vorstellen konnte und dann, als ich das aber hochgeladen habe, dann verstanden habe, also mit diesen ganzen komischen Rauten und Klammern und was weiss ich was. Aber das ging dann, das war dann in Ordnung $[\ldots] »(B 08,12)$.

Weitere Reflexionen beinhalteten die Beschreibungen von Erfolgserlebnissen, die sich dadurch einstellten, dass Studierende die Formatierung von Wikiseiten gelang. In entsprechenden Beschreibungen wurde die eigene Mitgestaltung eines Produktes im Internet positiv hervorgehoben. Eine Person ohne Erfahrungen mit Wikis beschrieb die gelungene Erstellung ihrer Wikiseite beispielsweise mehrfach als «schon ganz cool» (B09, 20):

«Also ich habe es halt auch persönlich auch noch nie gemacht, ich habe auch keine Ahnung von so was. Aber fand das überhaupt, ich fand das ganz cool auch mal selbst so was zu sehen: Ach ich habe jetzt gerade meine Überschrift hingekriegt. Also das war (Lachen) auch wenn es nur so kleine Erfolge waren, aber das war schon ganz cool irgendwo auch. Wo Birgit mir auch geschrieben hat, ich kriege es nicht hin, oder wir hatten dann auch mal etwas doppelt und wir haben es dann nicht mehr rausgelöscht bekommen und (kurze Pause) irgendwann ging es dann aber und man war dann auch nicht stolz auf sich aber schon: Okay wir haben es jetzt hingekriegt, das jetzt wirklich im Internet was von uns steht. Also es war schon ganz cool eigentlich» $(B 09,18)$.

Ferner sprach eine Person zum Abschluss des Interviews über die Möglichkeit der Zusammenarbeit durch Wikis (B11). Die Zusammenarbeit in ihrer Gruppe war über WhatsApp und das Verschicken von Word-Dateien organisiert. Die Person reflektierte die Schwierigkeiten dieser Vorgehensweisen und diskutierte alternative Handlungsoptionen zur Organisation der Gruppenarbeit. Dabei wurde die Zusammenarbeit 
mithilfe des Wikibooks als bessere und potentiell vorhandene Alternative beschrieben, welche jedoch im Rahmen des Seminars nicht genutzt wurde.

«[...] also, ich glaube wirklich, dass es im Endeffekt am besten gewesen wäre, hätten wir das im Vornherein in Wikibooks hochgeladen, jeder hätte sich einen Account gemacht und man hätte daran rumgebastelt, so gross die Gefahr auch ist, dass ein und dieselbe Stelle von sechs Leuten geändert wird, wäre das im Endeffekt besser gewesen als ständig diese Word-Dateien rum zu schicken. Also (kurze Pause) es kann, glaube ich, gut funktionieren, wenn sich die Gruppe einig ist, was für ein Medium benutzt wird oder, wenn einer halt die zündende Idee hat, wie man es halt machen kann oder man sich halt trifft. (Kurze Pause). Von daher fand ich das technisch eigentlich (kurze Pause) waren die Gegebenheiten schon da, das gut hinzukriegen, aber (Lachen) das ist so ein bisschen gescheitert halt so» (B11, 28).

Bei vergleichender Betrachtung der Interviews wird deutlich, dass die technische Organisation und Realisierung der Schreibprodukte von der Mehrheit der Studierenden mithilfe lokal gespeicherter Textverarbeitungsdokumente erfolgte. Die Möglichkeiten die Zusammenarbeit über die Plattform Wikibooks zu organisieren, wurde nur von einer Studierenden thematisiert. Von der Mehrheit der Studierenden wurde das Wikibook sowie der Wiki-Editor als Plattform zur Abgabe bzw. zum «Hochladen» der Ergebnisse beschrieben. Diese «Hochladen» wurde dabei häufig von einer bzw. einem Technikbeauftragten stellvertretend für die Gruppe erledigt.

\subsubsection{Wahrnehmung und Reflexion der (Wikibook-)Gemeinschaft}

Im Rahmen dieses Kapitels wird zwischen der Wahrnehmung und Reflexion der Seminargemeinschaft (7.4.1.4.1) sowie der Wahrnehmung und Reflexion der WikbookCommunity (7.4.1.4.2) unterschieden.

\subsection{Seminargemeinschaft}

Über die gemeinsame Erstellung des Wikibooks war es den Studierenden möglich, im Rahmen von Moodle sowie über das Wikibook die Schreibprodukte ihrer Mitstudierenden einzusehen und zu kommentieren. Mit dieser Möglichkeit sind die Studierenden in unterschiedlicher Weise umgegangen. Sieben Studierende (B01, B04, B07, B08, B09, B10, B11) beschrieben zunächst, dass sie sich mit den Texten ihrer Mitstudierenden auseinandergesetzt haben. Für zwei der Studierenden (B1, B04) eröffnete das Lesen der Textprodukte die Möglichkeit, nach den Zwischenpräsentationen in den Präsenzveranstaltungen der zweiten Seminarphase (Kap. 7.2.3.1) nachzusehen «was daraus geworden ist» (B01, 42). So beschrieben die zwei Studierenden, dass sie 
auf Basis der Präsentationen jeweils nur einen Einblick in die verschiedenen Themen bekamen. Das Nachlesen der Beiträge bot ihnen eine Möglichkeit, sich mit den Themen erneut auseinanderzusetzen und das eigene Verständnis zu prüfen: «[...] ich weiss ja, was inhaltlich in den Sitzungen passiert ist, theoretisch zumindest, wie genau man das noch weiss ist ja die Frage» (B01, 40). In ähnlicher Weise beschrieb eine weitere Studierende ihren Erkenntnisstand nach den Präsenzveranstaltungen und «von daher fand ich jetzt schön mal», so die Formulierung der Person, «das durchzulesen und mal zu schauen, wie haben das denn die anderen Gruppen so gemacht» (B04, 34). Diese Auseinandersetzung mit weiteren Beiträgen wurde von einer Person als regelmässige Aktivität beschrieben: «lch habe im Prinzip immer wenn ich gesehen habe, es ist etwas Neues da, habe ich es gelesen» (B01, 40). Eine weitere Studierende unterschied ihr Lesen der weiteren Beiträge hinsichtlich der unterschiedlichen Seminarphasen wie folgt:

«Also ich habe irgendwann mal auch in der Mittagspause oder sogar noch länger, hab mir mal drei, vier Stunden Zeit investiert und habe auch mal geguckt, was haben sich denn die anderen ausgedacht und haben uns das auch wirklich jetzt vielleicht nicht alles durchgelesen, aber halt schon diese Tabellen fand ich ganz cool bei diesen Metaphern, da haben wir uns das immer mal angeguckt oder halt dann auch mal wirklich weitergelesen, wenn es uns wirklich interessiert hat (kurze Pause). Jetzt bei den Denkwerkzeugen (kurze Pause) habe ich halt, also das habe ich auch schon viel gehört in anderen Seminaren und so. Deswegen habe ich da jetzt nicht so viel gelesen (kurze Pause), aber auch noch einmal geguckt teilweise, ob ich es richtig verstanden habe $[. .$.$] »$ (B09, 34).

Drei Studierende beschrieben die Auseinandersetzung mit den Beiträgen ihrer Mitstudierenden als Orientierungsmöglichkeit zur inhaltlichen Strukturierung der eigenen Beiträge (B08, B10, B11). Eine Studierende beschrieb die orientierende Funktion dahingehend, dass sie «gerade bei den Dimensionen geguckt haben: was haben die grob da eingeordnet? Also das hat uns eigentlich ganz gut geholfen $[\ldots]$ (B11, 36). In ähnlicher Weise beschrieb eine weitere Person, dass sie durch den Vergleich mit anderen studentischen Arbeitsgruppen versucht habe «Sicherheit zu bekommen» (B08, 46). Die Möglichkeit, die Schreibprodukte anderer vergleichen zu können, bewertete sie rückblickend «eigentlich ganz gut, weil es hat einem eigentlich nur geholfen, dass man auch andere Texte lesen kann und sehen kann, was die dazu beigetragen haben» (B08, 46). Auch die dritte Person beschrieb die Möglichkeit, andere Texte lesen zu können, als «sehr hilfreich» $(B 10,24)$. Dabei unterschied sie zwischen den zwei unterschiedlichen Phasen: 
"Jetzt gerade bei der zweiten Arbeitsphase, also zu den Theorien, habe ich schon genutzt, weil gerade durch die Rückmeldung, einfach durch das Feedback von euch man noch einmal die Möglichkeit hatte zu schauen, okay, wie haben die anderen das Problem gelöst. Das fand ich dann sehr hilfreich zu sehen: Okay, die haben das so gegliedert und haben in dem Bereich sehr viel geschrieben oder haben sich eher darauf konzentriert und auch einmal inhaltlich zu schauen: Okay, worum geht es bei denen und inwieweit könnte man das auch auf unser Projekt auch übertragen, also auf unser Thema. In diesem Fall fand ich es sehr gut zu schauen, bei den Metaphern habe ich eher, glaube ich, auf Formatsachen nachgeschaut bei den anderen, als inhaltlich mir wirklich etwas durchgelesen» (B10, 24).

Neben der inhaltlichen Orientierung wird in dem Zitat mit dem Begriff der «Formatsachen» auch die Möglichkeit angesprochen, sich hinsichtlich des Umgangs mit dem Wiki-Editor an weiteren Beiträgen zur orientieren. Diese Möglichkeit wurde, wie bereits skizziert (Kap. 7.4.1.3.3), auch von weiteren Studierenden genutzt (B07, B09).

In den Interviews mit vier weiteren Studierenden wurde nur eine geringe bis keine Auseinandersetzung mit den unterschiedlichen Beiträgen der Mitstudierenden beschrieben (B02, B03, B05, B06). Gemeinsam ist den Studierenden, dass sie jeweils über keine Vorerfahrungen in der Mitgestaltung von Wikis berichteten haben und sich in ihren Gruppen nicht aktiv mit dem Wiki-Editor auseinandersetzen mussten. Die Bewertung der Möglichkeit zum Lesen anderer Beiträge erfolgte von den Studierenden in unterschiedlicher Weise. Zwei Studierende (B02, B03) beschrieben ihre Auseinandersetzung mit anderen Beiträgen als «nur ein bisschen und nicht so intensiv» (B03, 156) bzw. als nicht wahrgenommen: «habe ich nicht genutzt» (B06, 70). Dies begründeten beide Personen damit, dass sie für eine Auseinandersetzung mit den entsprechenden Inhalten eher auf Primärliteratur zurückgreifen. Mit diesen Begründungen markierten die Studierenden Bedenken gegenüber den von Studierenden erstellten Beiträgen hinsichtlich der fachlichen Angemessenheit und Vertrauenswürdigkeit. Im Gegensatz dazu kamen die zwei weiteren Studierenden (B02, B05), die sich zum Zeitpunkt des Interviews ebenfalls «nicht wirklich» (B02) mit den Beiträgen ihrer Kommilitoninnen und Kommilitonen auseinandersetzt hatten, zu einer deutlich positiveren Einschätzung. In ihren Bewertungen zum Wikibook und der Möglichkeit, sich die Beiträge der Kommilitoninnen und Kommilitonen ansehen zu können, wurde nicht die fachliche Angemessenheit fokussiert, sondern die eigene Mitwirkung hervorgehoben:

«Aber, ich habe vor, mir das einmal im Ganzen, vielleicht nicht jeden Satz einzeln durchzulesen, aber auf jeden Fall mal anzusehen, wenn es fertig ist oder auch einmal keine Ahnung, um es meinen Eltern zu zeigen oder so, was wir da gemacht haben» $(B 02,46)$. 
«Also dieses Seminar finde ich richtig gut und ich habe auch sehr viel mitgenommen und jetzt weiss ich auch, wenn ich irgendetwas brauche über Lerntheorien, dann weiss ich, wo ich Zugriff habe direkt, und weiss auch das haben wir bearbeitet, das ist unsere Gruppe gewesen. Also fand ich echt sehr gut» (B05, 80).

Beim Vergleich der Interviews zeigt sich ein breites Spektrum an unterschiedlichen studentischen Perspektiven und Umgangsweisen mit den Textprodukten der Mitstudierenden. Die Möglichkeit, die Texte der anderen studentischen Arbeitsgruppen zu lesen, wurde beispielsweise von einer Person für alle neuen Beiträge wahrgenommen. Im Gegensatz dazu wurde in den Interviews aber auch das Vorgehen beschrieben, keine Texte der Mitstudierenden zu lesen.

\subsection{Wikbook-Community}

Im Rahmen des Wikibookprojektes konnten, wie in der Erprobung beschrieben (Kap. 7.2.2.3, 7.2.3.3, 7.2.4.3), Studierende unterschiedliche Erfahrungen in der Auseinandersetzung mit Mitgliedern der Wikicommunity machen. Diese Auseinandersetzungen und Interaktionen mit Wikibookianerinnen und Wikibookianern in Form von Kommentaren, Bearbeitungen und individuellen Nachrichten wurde von acht Studierenden explizit thematisiert (B01, B02, B04, B05 B06, B08, B09, B11). Drei weitere Studierende nahmen entsprechenden Interaktionen nicht wahr bzw. brachten diese im Interview nicht explizit zum Ausdruck (B03, B07, B10).

Die Wahrnehmung dieser Interaktionen sowie die damit verbundenen Einschätzungen und Reflexionen erfolgten mit unterschiedlichen Akzentuierungen. Für drei Studierende waren die Rückmeldungen nachvollziehbar, zudem äusserten sie sich wertschätzend gegenüber kritischen Kommentaren von Mitgliedern der Wikicommunity (B01, B04, B09, B11). Exemplarische Formulierungen sind z. B. «Also ich finde es gut, dass wir so jemanden haben» (B01, 48), oder «Gut, das finde ich eigentlich sehr sinnvoll und das sollte ja auch so sein. [...] also ich finde Feedback eigentlich immer produktiv» (B04, 38). Zugleich markierten vier Studierende, dass der Ton der Kritik unangemessen war bezeichneten diesen als «garstig» (B01), «scharf» (B02), «böse» und «bisschen fies» (B09) sowie als «unfreundlich» (B11). Für drei Studierende (B01, $B 02, B 09)$ blieb die Kritik dennoch berechtigt, «aber man kann auch ein bisschen netter sein an manchen Stellen» (B01, 48). Zwei weitere Studierende (B02, B11) markierten die Art und Weise der kritisierten Aspekte jedoch als problematisch: «Was ich aber als negativ empfunden habe, was ich wirklich mal sagen muss, was uns in der Gruppe auch echt aufgeregt hat, war dieser Jürgen» (B11, 14). Dies begründete die Person mit unterschiedlichen Argumenten, z. B. fehlten der Person Begründungen für die kritisierten Aspekte: 
"Aber er sagt halt auch selten warum. Also ich finde einfach, er sagt, er mag das nicht und dann (kurze Pause) war es das. Und dann sagt dieser andere Typ halt auch noch einmal: Ne ist blöd. Oder sagt halt: Ja ist gut» (B11, 46).

Darüber hinaus wurden von den Studierenden sehr unterschiedliche Aspekte hervorgehoben. Drei Studierende diskutierten die Frage, inwiefern Wikibookianerinnen und Wikibookianer wie Juetho qualifiziert sind, um inhaltliche Rückmeldungen zu geben (B02, B05, B11). So lautete die Formulierung einer Person: «Weil ich mich im Endeffekt gefragt, habe inwiefern dieser Jürgen überhaupt qualifiziert ist, bei unserem Pädagogik Wikibook irgendetwas zu sagen. Also was jetzt nicht die äussere Form betrifft $[. .] ».(B 11,14)$. Eine weitere Person markierte, dass die Kommentare einen Anlass darstellten, Aussagen zu prüfen:

«Genau. Wenn er jetzt gesagt hätte, das ist inhaltlich nicht so gut, dann hätte ich trotzdem noch einmal geschaut, und wenn ich der Meinung bin das ist inhaltlich gut, dann hätte ich das auch so gelassen» $(\mathrm{B} 05,62)$.

Weitere Reflexionen widmeten sich der Frage, warum sich Wikibookianerinnen und Wikibookianer so intensiv mit dem im Seminar erstellten Wikibook auseinandersetzten (B02, B09, B11). Eine Person fragte sich beispielsweise, «ob er jetzt vielleicht der Gründer oder so von der Seite ist» (B09, 28). Zwei weitere Studierende thematisierten, dass die Kommentare von Juetho hilfreich waren, um die angemessene Nutzung von Wikibooks zu erlernen (B01, B11). So wurden die Kommentare von Juetho zur Formatierung als sinnvolles Korrektiv beschrieben $(B 01,48)$ bzw. erläutert, dass bestimmte Kommentare von Juetho dazu führten, dass die Studierenden für die Anmeldung bei Wikibooks sensibilisiert wurden und versuchten, nicht mehr anonym ihre Seiten zu bearbeiten (B11, 30).

Eine Person, welche sich bereits zu Beginn des Interviews uninteressiert gegenüber der Projektidee geäussert hatte, markierte, dass sie die Kommentare zwar einerseits wahrgenommen habe. Andererseits hatten diese sie «nicht gestört ehrlich gesagt, also ging mir jetzt nicht besonders Nahe oder so» (B06, 72). Im Gegensatz dazu waren die Kommentare für eine andere Person, die sich zu Beginn des Interviews von der Idee der Veröffentlichung distanziert hatte, von Bedeutung. Für diese waren die Kommentare der Wikibookianerinnen und Wikibookianer ein Indikator dafür, dass die Texte wirklich gelesen werden. Dies hat zugleich ihre «Ängste bestätigt», so die Formulierung dieser Person, «weil ich eben bemerkt habe: Ja das lesen wirklich andere und mehrere und Menschen, die ich gar nicht kenne, die damit gar nichts zu tun haben» $(\mathrm{B} 08,10)$.

In drei Interviews wurden von den Studierenden Interaktionen mit den Wikibookianerinnen und Wikibookianern nicht explizit zum Ausdruck gebracht (B03, B07, B10). Die Nichtthematisierung ist zum einen interessant, da sich eine Person 
skeptisch gegenüber der Reichweite von Wikibooks äusserte «also ich glaube jetzt auch ehrlich gesagt nicht, dass da so viele Leute da recherchieren» (B03, 32). In diesem Sinne vertrat die Person auch die Meinung, dass sie wohl kaum Rückmeldungen auf Beiträge erhalten wird: «und dann höre ich wahrscheinlich erst mal nichts, wenn es nicht ganz extrem ist» $(\mathrm{B} 03,36)$. Auf die Frage, ob die Person bereits die Kommentare zum Wikibook wahrgenommen habe, verneinte sie: «Ne, ehrlich gesagt nicht so. $\mathrm{Ne}$, also» (B03, 38). Zudem thematisierten Studierende die Möglichkeiten zur Veränderungen der geschriebenen Texte (B07, B08, B10, B11). Dabei problematisierten insbesondere die Studierenden, welche die Bearbeitungen nicht wahrnahmen, dass es die Möglichkeit zur Bearbeitung des «eigenen» Textes gibt.

"Das ist bei uns, soweit ich weiss, nicht passiert eigentlich. Dass die Möglichkeit besteht, das finde ich ehrlich gesagt nicht so toll. Also zumindest nicht einfach so, also da sollte man schon eigentlich irgendwie mal darüber Bescheid wissen. Also zumindest, dass man weiss, ja, dass da jemand an dem Text rumwerkelt, weil es ist ja schon der eigene [Text]» (B07, 54).

Beim Vergleich der Interviews zeigt sich, dass Interaktionen und Kommentare von Wikibookianerinnen und Wikibookianern von mehreren Studierenden wahrgenommen und im Rahmen der Interviews thematisiert wurden. Die Einschätzung der Kommentare war für die entsprechenden Studierenden weitgehend nachvollziehbar. Verschiedene Studierenden problematisierten den «Ton» der formulierten Kritik. Zudem wurde sichtbar, dass verschiedene Studierende die Partizipations- und Kollaborationsmöglichkeiten nicht kannten bzw. erst im Verlauf des Interviews realisierten.

\subsubsection{Zusammenfassung und Relevanz der Interviewergebnisse}

Die Ergebnisse der Interviewanalyse umfassen studentische Positionen zu vier zentralen Themen: Einstellungen gegenüber der Projektidee (Kap. 7.4.1.1), Vorkenntnisse und Vorerfahrungen der Studierenden (Kap. 7.4.1.2), Strategien zur Zusammenarbeit und zum Medienhandeln (Kap. 7.4.1.3) sowie Wahrnehmung und Reflexion der (Wikibook-)Gemeinschaft (Kap. 7.4.1.4). Mit unterschiedlichen Begründungen, z. B. Abwechslung gegenüber anderen Seminarerfahrungen, äusserten sich mehrere Studierende positiv gegenüber der Projektidee (Kap. 7.4.1.1). Vorerfahrungen im Umgang mit Wikis hatte die Mehrheit der Studierenden hinsichtlich rezeptiver Nutzungsstrategien zur Recherche von Informationen. Zugleich hatte die Mehrheit der Studierenden keine Vorerfahrungen bzw. Vorkenntnisse hinsichtlich der Mitgestaltung von Wikiprojekten (Kap. 7.4.1.2). Die gemeinsame Erstellung von Texten erfolgte in kollaborativen, kooperativen sowie kollektiven Formen der Zusammenarbeit. Die Möglichkeiten die Zusammenarbeit über die Plattform Wikibooks zu organisieren, wurde nur von einer Studierenden thematisiert. Für die Mehrheit der Studierenden 
wurde Wikibook stattdessen vor allem als Plattform zum Hochladen der Ergebnisse beschrieben. Das Hochladen wurde in verschiedenen Gruppen nur von einzelnen Personen übernommen (Kap. 7.4.1.3). Die Kommentare der Wikicommunity wurden von verschiedenen Studierenden wahrgenommen und im Rahmen der Interviews unterschiedlich thematisiert. Während die formulierten Kritikpunkte für die Studierenden in der Regel nachvollziehbar waren, problematisierten sie den «Ton» der Kritik.

Auf Basis der strukturierenden Inhaltsanalyse der Interviews konnten verschiedene empirische Indikatoren identifiziert werden, welche neue Erfahrungsmöglichkeiten von Studierenden zum Lernen mit digitalen Medien zeigen (Kap. 3.2.1.3) sowie Hinweise zu potenziellen Anlässen zum Lernen über soziale Medien eröffnen (Kap. 3.2.1.2). Relevante Aspekte der Interviewauswertung werden im folgenden Kapitel Auswertung und Neuperspektivierung (Kap.8) aufgegriffen und zur Modifikation des Entwurfs diskutiert (Kap. 9).

\subsubsection{Ergebnisse der Veranstaltungsevaluation}

Die Durchführung der Veranstaltungsevaluation dient dem Ziel, die Perspektive der Studierenden zu ausgewählten Seminarelementen systematisch zu erfassen. Neben der studentischen Einschätzung des erlebten Seminars (Kap. 6.6) wird die Absicht verfolgt, Erkenntnisse über die Erfahrungsmöglichkeiten zum Lernen mit sozialen Medien (Kap. 3.2.1.3) zu gewinnen. Ferner sollen potenzielle Lernsituationen zur Teilhabe an öffentlichen Wikis (Kap. 3.2.1.2) auf Basis der Evaluation näher bestimmt werden. Dafür wurden im Rahmen der letzten Präsenzveranstaltungen offene Gruppengespräche geführt (Kap. 3.3.3.1, 7.3.2.2) und ein Evaluationsbogen verwendet (Kap. 3.3.3.2, 7.3.2.1). Zur Darstellung der Ergebnisse wird zwischen der Auswertung der quantitativen Evaluationsdaten (Kap. 7.4.2.1) sowie der Auswertung der qualitativen Evaluationsdaten (Kap. 7.4.2.2) unterschieden.

\subsubsection{Auswertung der quantitativen Evaluationsdaten}

Die quantitative Evaluation der zwei Lehrveranstaltungen ( $N=19)$ eröffnet einen Einblick in die studentische Perspektive zum Erleben sowie zur Bewertung des Wikibookprojektes (Kap. 7.3.2.1). Die Evaluationsdaten stammen aus den zwei inhaltlich unterschiedlichen Seminaren «Lehren und Lernen metaphorisch verstehen» und «Bildung metaphorisch verstehen», welche auf Basis des gleichen Konzepts geplant und durchgeführt worden sind (Kap. 6, 7.2). Zur Darstellung der Ergebnisse werden vier thematische Aspekte unterschieden:

- Einschätzung der Lehrveranstaltung (Kap. 7.4.2.1.1)

- Bewertung der Seminaranforderungen und Lehr-Lerninteraktionen (Kap. 7.4.2.1.2)

- Bewertung der Erfahrungen zur Mitgestaltung eines Wikibooks (Kap. 7.4.2.1.3) 
Die Darstellung der Ergebnisse erfolgt in Form von Häufigkeitstabellen (Kap. 3.4.2.1). Zentrale Tendenzen der Antworthäufigkeiten werden durch die Zusammenfassung jeweils zweier Antwortkategorien dargestellt ${ }^{215}$.

\subsection{Einschätzung der Lehrveranstaltung}

Der erste thematische Schwerpunkt umfasst Aussagen zur gesamten Lehrveranstaltung $^{216}$. Dazu zählen Formulierungen zum erlebten Seminarkonzept über den Verlauf des Semesters (Tab. 7.9) sowie eine abschliessende Bewertung des Seminars mit Schulnoten. Die Bewertung der Studierenden fällt überwiegend positiv aus.

Die positive Bewertung zeigt sich zum einen in der Einschätzung des Seminarkonzeptes und der Einschätzung der Organisation (Tab. 7.9). Die häufigste Zustimmung erhielten die formulierten Aussagen zum angemessenen Tempo (57,9 \%), zur guten Strukturierung des Seminars (50\%) sowie zum angemessenen Wechsel zwischen Präsenz- und Schreibphasen (55,6 \%). In der Zusammenfassung der zwei Antwortkategorien «Stimme zu» und «Stimme eher zu» wurde den drei Aussagen jeweils von über 80 \% der Evaluationsteilnehmerinnen und -teilnehmer (eher) zugestimmt. Die Aussagen zur Angemessenheit des inhaltlichen Umfangs sowie zur ausreichenden Zeit zur Diskussion bewerteten jeweils über $50 \%$ der Evaluationsteilnehmerinnen und -teilnehmer (eher) positiv. Zugleich war die Anzahl an nicht zustimmenden Aussagen sehr gering.

215 Dafür werden sprachlich die zwei Antwortkategorien «Stimme zu» und «Stimme eher zu» zu «Stimme (eher) zu» zusammengefasst. Entsprechend werden sprachlich die zwei Antwortkategorien «Stimme nicht zu» und «Stimme eher nicht zu» zu «Stimme (eher) nicht zu» zusammengefasst.

216 Für Wildt $(2002,8)$ umfasst diese didaktische Handlungsebene einzelne Lehrveranstaltungen bzw. die «Zusammenfassung [...] aufeinander folgender Lernsituationen». 


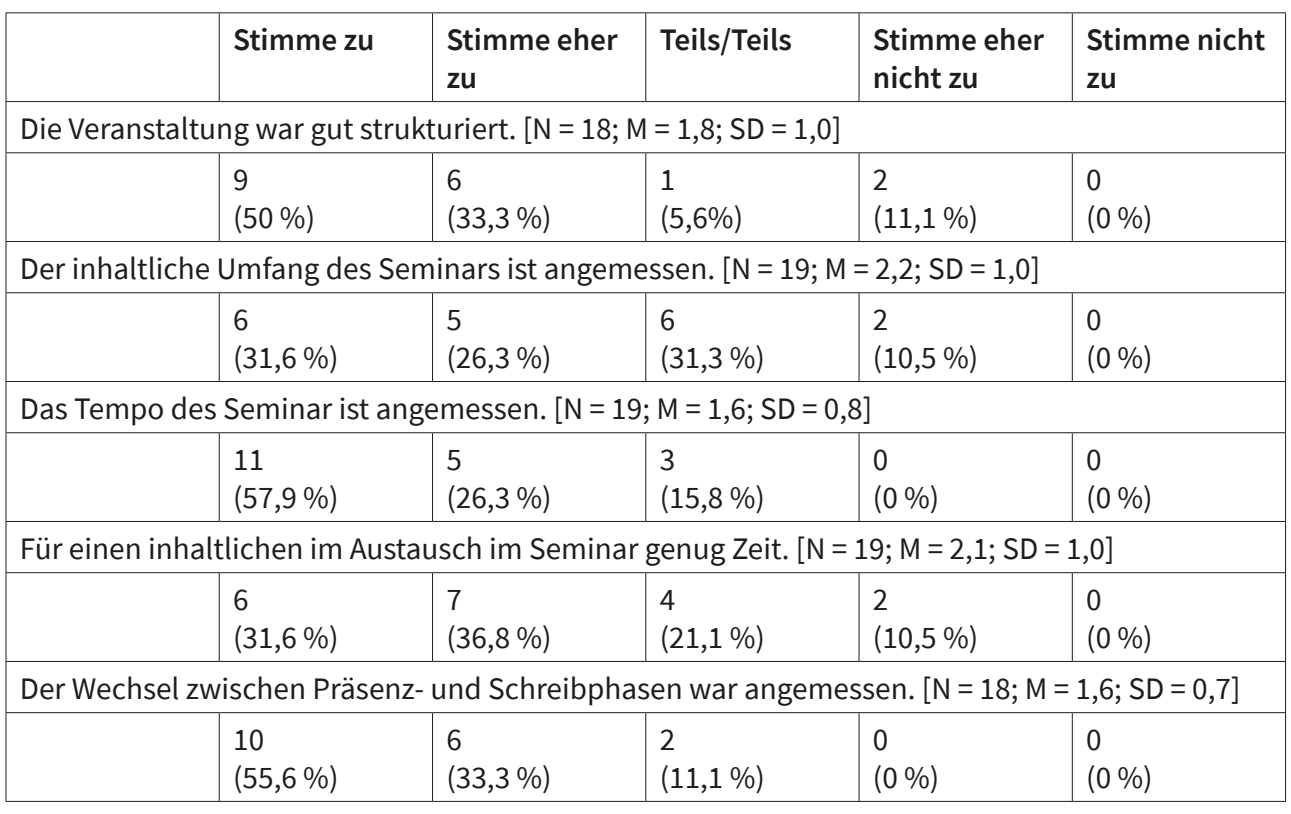

Tab. 7.9: Einschätzung des Seminarkonzeptes und der Organisation durch die Studierenden.

Die positive Bewertung zeigt sich zum anderen in der Benotung des Seminars durch die Studierenden. 14 von 18 Studierenden vergaben die Note "Gut» oder «Sehr gut». Vier Studierende bewerteten die Veranstaltung mit befriedigend. Der Mittelwert mit $M=2,1$ sowie die geringe Standardabweichung $S D=0,6$ bestätigen den Schwerpunkt der Häufigkeitsverteilung bei der Note «Gut».

\subsection{Bewertung der Seminaranforderungen und Lehr-Lerninteraktionen}

Die Einschätzung der Seminaranforderungen sowie der Lehr-Lerninteraktionen stellt den zweiten thematischen Schwerpunkt der Ergebnisdarstellung dar. Dafür wird die Perspektive der Studierenden hinsichtlich dreier Schwerpunkte auf der Ebene von Lernsituationen dargestellt: Transparenz der Ziele und Einschätzung der Leistungsanforderungen (Tab. 7.10) sowie ihrer Zufriedenheit mit der Gruppenbildung, der Unterstützung der Gruppenaktivitäten und der Aktivität der Gruppenteilnehmerinnen und -teilnehmer (Tab. 7.11). Zudem werden die Aussagen der Studierenden zu LehrLern-Interaktionen dargestellt. Dies erfolgt hinsichtlich ihrer Einschätzung der Interaktionen in der Präsenz (Tab. 7.12) und durch die Betrachtung ihrer Einschätzung der schriftlichen Rückmeldungen und Hinweise via Moodle (Tab. 7.13).

Aussagen zur Transparenz der Zielsetzung sowie der zu erbringenden Leistungen wurden überwiegend zustimmend bewertet (Tab. 7.10). Etwa zwei Drittel der Teilnehmerinnen und Teilnehmer beantworteten die Aussagen jeweils mit «Stimme zu» oder mit «Stimme eher zu». Lediglich einer Person sind Lehrziele der Veranstaltung 
in der ersten Blockveranstaltung eher nicht klargeworden. Im Kontrast zu «ReferatSeminaren» wurden Studierende im Rahmen der Veranstaltung mit der Aufgabe konfrontiert, Textprodukte auf unterschiedlichen Abstraktionsebenen für ein öffentliches Wikibookprojekt zu formulieren. Während die Formulierung von Hausarbeiten sowie die schriftliche Auseinandersetzung mit Themen für Studierende erfahrbar wird, beschränken sich entsprechende Aufgabenformate häufig auf individuelle Leistungen, die nicht im Rahmen der Vorlesungszeit bzw. konkreter Seminare entstehen müssen. Die mögliche Problematik, dass die entsprechenden komplexen Aufgaben zur Texterstellung für die Studierenden eine Überforderung darstellen, wurde aus der Perspektive der Teilnehmerinnen und Teilnehmer der Evaluation nicht markiert (Tab. 7.10). Jeweils über $80 \%$ der Teilnehmerinnen und Teilnehmer stimmten den Aussagen (eher) nicht zu, dass die eigenständige Textproduktion sowie das wissenschaftliche Schreiben von (Fach-)Texten eine Überforderung waren. Dies zeigt sich auch in den hohen Mittelwerten von $M=4,3$ zum Schreiben wissenschaftlicher Texte sowie $M=4,4$ hinsichtlich der eigenständigen Textproduktion ${ }^{217}$.

\begin{tabular}{|c|c|c|c|c|c|}
\hline & Stimme zu & $\begin{array}{l}\text { Stimme eher } \\
\mathrm{zu}\end{array}$ & Teils/Teils & $\begin{array}{l}\text { Stimme eher } \\
\text { nicht zu }\end{array}$ & $\begin{array}{l}\text { Stimme nicht } \\
\mathrm{zu}\end{array}$ \\
\hline \multicolumn{6}{|c|}{$\begin{array}{l}\text { Die Lehrziele der Veranstaltung sind mir in der ersten Blickveranstaltungen klar geworden. [N } \\
=19, M=2,1, S D=0,9]\end{array}$} \\
\hline & $\begin{array}{l}5 \\
(27,8 \%)\end{array}$ & $\begin{array}{l}7 \\
(38,3 \%)\end{array}$ & $\begin{array}{l}5 \\
(27,8 \%)\end{array}$ & $\begin{array}{l}1 \\
(5,6 \%) \\
\end{array}$ & $\begin{array}{l}0 \\
(0 \%)\end{array}$ \\
\hline \multicolumn{6}{|c|}{$\begin{array}{l}\text { Die Anforderungen an den Leistungserwerb wurden transparent gemacht. [N=19; } M=1,8 ; S D= \\
0,9]\end{array}$} \\
\hline & 9 & $\begin{array}{l}5 \\
(26,3 \%)\end{array}$ & $\begin{array}{l}5 \\
(26,3 \%)\end{array}$ & $\begin{array}{l}0 \\
(0 \%)\end{array}$ & $\begin{array}{l}0 \\
(0 \%)\end{array}$ \\
\hline \multicolumn{6}{|c|}{$\begin{array}{l}\text { Die Anforderung, eigenständig Wissen in Form von Texten produzieren zu müssen, war eine } \\
\text { Überforderung. [N=18; } M=4,4 ; S D=0,7]\end{array}$} \\
\hline & $\begin{array}{l}0 \\
(0 \%)\end{array}$ & $\begin{array}{l}0 \\
(0 \%)\end{array}$ & $\begin{array}{l}3 \\
(16,7 \%)\end{array}$ & $\begin{array}{l}7 \\
(38,9 \%)\end{array}$ & $\begin{array}{l}8 \\
(44,4 \%)\end{array}$ \\
\hline \multicolumn{6}{|c|}{$\begin{array}{l}\text { Das wissenschaftliche Schreiben von (Fach-)Texten war eine Überforderung. [N=18; } M=4,3 ; S D \\
=0,8]\end{array}$} \\
\hline & $\begin{array}{l}0 \\
(0 \%)\end{array}$ & $\begin{array}{l}0 \\
(0 \%)\end{array}$ & $\begin{array}{l}3 \\
(16,7 \%)\end{array}$ & $\begin{array}{l}7 \\
(38,9 \%)\end{array}$ & $\begin{array}{l}8 \\
(44 \%)\end{array}$ \\
\hline
\end{tabular}

Tab. 7.10: Einschätzung der Transparenz der Ziele und der Leistungsanforderungen durch die Studierenden.

Zur Strukturierung und Unterstützung der Studierenden in den Gruppenphasen wurden zum einen Angebote in der Präsenz sowie über Moodle ${ }^{218}$ geschaffen. Zum

217 Zugleich finden sich in den Äusserungen der Gruppengespräche auch Hinweise darauf, dass die Anforderungen des Seminars für einige Studierende herausfordernd bzw. überfordernd erschienen (Kap. 7.4.2).

218 Die Nutzung der Moodle-Foren wurde in den Kapiteln 7.2.2.2, 7.2.3.2 und 7.2.4.2 beschrieben. 
anderen wurden die Gruppenbildungsprozesse didaktisch unterstützt (Kap. 6.6.1, 7.2.2, 7.2.2). Die verschiedenen Orientierungs- und Unterstützungsangebote wurden von der Mehrheit der Studierenden positiv bewertet (Tab. 7.11). Über $60 \%$ der Studierenden stimmten (eher) der Aussage zu, dass das Seminar genügend Unterstützung zur Gruppenorganisation und zum Schreiben bot. Nur zwei von 18 Studierenden stimmten der Aussage eher nicht zu. Die verschiedenen Ansätze zur Gruppenbildung im Rahmen der Auftaktsitzung (Kap. 7.2.2) sowie zu Beginn der zweiten Projektphase (Kap. 7.2.3) wurden jeweils von über $50 \%$ der Evaluationsteilnehmerinnen und -teilnehmer - in der Zusammenfassung der Antwortkategorien «Stimme zu» und «Stimme eher zu»- als (eher) angemessen bewertet. Zugleich zeigt sich auch, dass die Aussage zur Angemessenheit der Gruppeneinteilung in der zweiten Phase seltener positiv zugestimmt wurde und das Antwortspektrum vielfältiger ausfiel. Diese Unterschiede werden auch bei Betrachtung der Mittelwerte bzw. der Standardabweichung sichtbar. Die Aussage zur unterstützenden Funktion von Moodle für die Interaktion der Studierenden hat im Vergleich zu den vorherigen Aussagen die geringste Zustimmung erhalten. Neun von 18 Studierenden stimmten der Aussage (eher) zu, während 3 Studierende der Aussage (eher) nicht zustimmten. 


\begin{tabular}{|l|l|l|l|l|l|}
\hline & Stimme zu & $\begin{array}{l}\text { Stimme eher } \\
\text { zu }\end{array}$ & Teils/Teils & $\begin{array}{l}\text { Stimme eher } \\
\text { nicht zu }\end{array}$ & $\begin{array}{l}\text { Stimme nicht } \\
\text { zu }\end{array}$ \\
\hline
\end{tabular}

Das Seminar bot genügend Unterstützung zur Organisation der Gruppenarbeiten und das gemeinsame Schreiben. $[N=18 ; M=2,2 ; S D=1,0]$

\begin{tabular}{|l|l|l|l|l|l|}
\hline & 5 & 6 & 5 & 2 & 0 \\
$(27,8 \%)$ & $(33,3 \%)$ & $(27,8 \%)$ & $(11,1 \%)$ & $(0 \%)$ \\
\hline
\end{tabular}

Der Moodlekurs des Seminares hat die Interaktionen zwischen den Studierenden angemessen unterstützt. [N=18; $M=2,6 ; S D=1,1]$

\begin{tabular}{|l|l|l|l|l|l|}
\hline & $\begin{array}{l}9 \\
(50 \%)\end{array}$ & $\begin{array}{l}4 \\
(22,2 \%)\end{array}$ & $\begin{array}{l}4 \\
(22,2 \%)\end{array}$ & $\begin{array}{l}1 \\
(5,6 \%)\end{array}$ & $\begin{array}{l}0 \\
(0 \%)\end{array}$ \\
\hline
\end{tabular}

Die Gruppeneinteilung in der ersten Phase („Sympathigruppen“) war angemessen. $[\mathrm{N}=19 ; \mathrm{M}=1,8 ; \mathrm{SD}=1,0]$

\begin{tabular}{|l|l|l|l|l|l|}
\hline & 8 & 3 & 3 & 1 & 4 \\
$(42,1 \%)$ & $(15,8 \%)$ & $(15,8 \%)$ & $(5,3 \%)$ & $(21,1 \%)$ \\
\hline
\end{tabular}

Die Gruppeneinteilung in der zweiten Phase („Mischgruppen“) war angemessen. $[\mathrm{N}=19 ; \mathrm{M}=2,5 ; \mathrm{SD}=1,6]$

\begin{tabular}{|l|l|l|l|l|l|}
\hline & 8 & 3 & 3 & 1 & 4 \\
$(42,1 \%)$ & $(15,8 \%)$ & $(15,8 \%)$ & $(5,3 \%)$ & $(21,1 \%)$ \\
\hline
\end{tabular}

Ich bin mit der Aktivität meiner Kommilitonen in der ersten Gruppenphase (Entwicklung von Metaphern) zufrieden. [ $\mathrm{N}=19 ; \mathrm{M}=2,1 ; \mathrm{SD}=1,2]$

\begin{tabular}{|c|c|c|c|c|c|}
\hline & 8 & $\begin{array}{l}4 \\
(21,1 \%)\end{array}$ & $\begin{array}{l}5 \\
(26,3 \%)\end{array}$ & $\begin{array}{l}1 \\
(5,3 \%)\end{array}$ & $\begin{array}{l}1 \\
(5,3 \%)\end{array}$ \\
\hline \multicolumn{6}{|c|}{$\begin{array}{l}\text { Ich bin mit der Aktivität meiner Kommilitonen in der zweiten Gruppenphase (Entwicklung vor } \\
\text { Denkwerkzeugen) zufrieden. }[N=19 ; M=2,8 ; S D=1,4]\end{array}$} \\
\hline & $\begin{array}{l}3 \\
(15,8 \%)\end{array}$ & $\begin{array}{l}7 \\
(36,8)\end{array}$ & $\begin{array}{l}3 \\
(15,8 \%)\end{array}$ & $\begin{array}{l}3 \\
(15,8 \%)\end{array}$ & $\begin{array}{l}3 \\
(15,8 \%)\end{array}$ \\
\hline
\end{tabular}

Tab. 7.11: Zufriedenheit der Studierenden mit der Gruppenbildung, der Unterstützung der Gruppenaktivitäten und der Aktivität der Gruppenmitglieder.

Von den Studierenden wurde erwartet, gemeinsam in Gruppen spezifische Textprodukte zu erstellen (Kap. 6.6.1.1, 6.6.1.2). Die Zufriedenheit der Studierenden mit der Aktivität der Gruppenmitglieder in der ersten und zweiten Projektphase unterscheidet sich (Tab. 7.11). Wenngleich jeweils über $50 \%$ der Evaluationsteilnehmerinnen und -teilnehmer mit der Aktivität ihrer Gruppenmitglieder in beiden Phasen (eher) zufriedenen waren, steigerte sich die Anzahl der unzufriedenen Studierenden. Während in der ersten Gruppenarbeitsphase lediglich zwei von 19 Studierenden mit der Aktivität ihrer Mitstudierenden (eher) nicht zufrieden waren, waren es in der zweiten Gruppenarbeitsphase sechs von 19 Studierenden.

Die zwei weiteren thematischen Bereiche umfassen Interaktionen der Präsenzveranstaltungen (Kap. 7.2.2.1, 7.2.3.1, 7.2.4.1) sowie die Rückmeldungen und Literaturhinweise via Moodle (Kap. 7.2.2.2, 7.2.3.2). Bei der Bewertung der Präsenzsitzungen wiederholen sich - in Ansätzen - die Ergebnisse zur Zufriedenheit der Studierenden mit ihren Kommilitoninnen und Kommilitonen in der zweiten Gruppenphase 
(Tab. 7.12). Bei einem Mittelwert von $M=2,8$ waren acht Studierende mit der Aktivität ihrer Kommilitoninnen und Kommilitonen (eher) zufrieden während fünf Studierende (eher) nicht zufrieden waren. Die auffällige Streuung der Antworten wird auch durch den hohen Wert der Standardabweichung SD = 1,3 sichtbar. Deutlich positiver bewerteten die Studierenden die eigene Beteiligung am Seminargeschehen sowie die Aktivität der Lehrperson. Es stimmten mindestens 14 von 19 Studierende (d. h. jeweils über $70 \%$ ) den zwei Aussagen zu.

\begin{tabular}{|c|c|c|c|c|c|}
\hline & Stimme zu & $\begin{array}{l}\text { Stimme eher } \\
\text { zu }\end{array}$ & Teils/Teils & $\begin{array}{l}\text { Stimme eher } \\
\text { nicht zu }\end{array}$ & $\begin{array}{l}\text { Stimme nicht } \\
\text { zu }\end{array}$ \\
\hline \multicolumn{6}{|c|}{ Die Lehrperson regt uns in der Präsenz gezielt zum Nachdenken an. $[N=19 ; M=1,8 ; S D=0,9]$} \\
\hline & $\begin{array}{l}8 \\
(42,8 \%)\end{array}$ & $\begin{array}{l}7 \\
(36,8 \%)\end{array}$ & $\begin{array}{l}3 \\
(15,8 \%)\end{array}$ & $\begin{array}{l}1 \\
(5,3 \%)\end{array}$ & $\begin{array}{l}0 \\
(0 \%)\end{array}$ \\
\hline \multicolumn{6}{|c|}{ Am Seminargeschehen habe ich mich beteiligt. $[N=19 ; M=2,0 ; S D=0,8]$} \\
\hline & $\begin{array}{l}6 \\
(31,6 \%)\end{array}$ & $\begin{array}{l}8 \\
(42,1 \%)\end{array}$ & $\begin{array}{l}5 \\
(26,3 \%)\end{array}$ & $\begin{array}{l}0 \\
(0 \%)\end{array}$ & $\begin{array}{l}0 \\
(0 \%)\end{array}$ \\
\hline \multicolumn{6}{|c|}{$\begin{array}{l}\text { Ich bin mit der Aktivität meiner Kommilitonen im Rahmen der Präsenztermine zufrieden. } \\
{[N=19 ; M=2,8 ; S D=1,3]}\end{array}$} \\
\hline & $\begin{array}{l}3 \\
(15,8 \%)\end{array}$ & $\begin{array}{l}5 \\
(25,3 \%)\end{array}$ & $\begin{array}{l}6 \\
(31,6 \%)\end{array}$ & $\begin{array}{l}2 \\
(10,5 \%)\end{array}$ & $\begin{array}{l}3 \\
(15,8 \%)\end{array}$ \\
\hline
\end{tabular}

Tab. 7.12: Einschätzung der Interaktionen in der Präsenz durch die Studierenden.

Eine eher positive Bewertung der Interaktionen mit den Lehrenden zeigt sich auch in der Bewertung der schriftlichen Rückmeldungen und Literaturempfehlungen via Moodle (Tab. 7.13). So stimmten 14 von 19 Studierenden den zwei Aussagen (eher) zu, dass die Rückmeldungen für die Metaphernformulierungen nachvollziehbar waren und die Literaturempfehlungen zur Auseinandersetzung mit einer Theorie bzw. einem Modell hilfreich waren. Die Rückmeldungen zu den Textprodukten der zweiten Gruppenphase bewerteten 12 von 19 Studierenden als nachvollziehbar.

Bei zusammenfassender Betrachtung der studentischen Perspektive zur Bewertung von Lernsituationen und Interaktionen sind folgende Aspekte zentral: Es zeigt sich zunächst, dass die Studierenden die Lehrziele und Leistungsanforderungen verstanden haben und sich nicht von den Schreibaufgaben überfordert fühlten (Tab. 7.10). Bei der Bearbeitung in Gruppen gab es zugleich Studierende, die mit der Gruppenbildung in der zweiten Phase unzufrieden waren sowie mit der Aktivität ihrer Mitstudierenden (Tab. 7.11). Es zeigt sich, dass es bei den jeweiligen Fragen eine hohe Streuung bei den Antworten gibt. In den zwei weiteren Fragegruppen bewerteten die Studierenden die online erhaltenen Rückmeldungen als nachvollziehbar (Tab. 7.13) und empfanden die Aktivität des Lehrenden in den Präsenzveranstaltungen als fördernd (Tab. 7.13). 


\begin{tabular}{|l|l|l|l|l|l|}
\hline & Stimme zu & $\begin{array}{l}\text { Stimme eher } \\
\text { zu }\end{array}$ & Teils/Teils & $\begin{array}{l}\text { Stimme eher } \\
\text { nicht zu }\end{array}$ & $\begin{array}{l}\text { Stimme nicht } \\
\text { zu }\end{array}$ \\
\hline $\begin{array}{l}\text { Die schriftliche Rückmledung für unsere Metapher war für mich nachvollziehbar. } \\
\text { [N = 19; M =1,8; SD =0,9] }\end{array}$ \\
\hline $\begin{array}{l}9 \\
(47,4 \%)\end{array}$ & $\begin{array}{l}5 \\
(26,3 \%)\end{array}$ & $\begin{array}{l}5 \\
(26,3 \%)\end{array}$ & $\begin{array}{l}0 \\
(0 \%)\end{array}$ & $\begin{array}{l}0 \\
(0 \%)\end{array}$ \\
\hline $\begin{array}{l}\text { Die Literaturempfehlung waren hilfreich zur Auseinandersetzung mit unserer Theorie/unserem } \\
\text { Modell [N = 19; M=2,0; SD = 0,9] }\end{array}$ & $\begin{array}{l}6 \\
(31,6 \%)\end{array}$ & $\begin{array}{l}4 \\
(42,1 \%)\end{array}$ & $\begin{array}{l}1 \\
(5,3 \%)\end{array}$ & $\begin{array}{l}0 \\
(0 \%)\end{array}$ \\
\hline
\end{tabular}

Tab. 7.13: Einschätzung der schriftlichen Rückmeldungen und Hinweise via Moodle durch die Studierenden.

\subsection{Bewertung der Erfahrungen zur Mitgestaltung eines Wikibooks}

Die Einschätzung und Bewertung der Wikibookerfahrungen stellen den dritten thematischen Schwerpunkt der Ergebnisdarstellung der quantitativen Evaluation dar. Dafür wird die studentische Perspektive hinsichtlich der folgenden Facetten präsentiert: Erfahrungen mit Wikibooks und Bedeutung des öffentlichen Schreibens (Tab. 7.14), Interesse und Einschätzung der öffentlichen Rückmeldungen und Texte der Mitstudierenden (Tab. 7.15) sowie Bedienbarkeit des Wiki-Editors und Bewertung der Unterstützungsangebote (Tab. 7.16).

An frei und öffentlich zugänglichen Texten (mit) zu schreiben, war für über 80 $\%$ der Teilnehmerinnen und Teilnehmer der Evaluationssitzung etwas Neues (Tab. 7.14). Der Aussage, dass die im Seminar produzierten Texte von Menschen ausserhalb des Seminars gelesen werden können, das eigene Schreiben beeinflusst, stimmten 12 von 17 Studierenden (eher) nicht zu. Weniger eindeutig wurde die Aussage bewertet, inwiefern das Schreiben an Wikitexten eine besondere Bedeutung für die Studierenden hat. Bei einem Mittelwert von $M=3,0$ stimmten acht Studierende der Aussage (eher) zu, während fünf Studierende der Aussage (eher) nicht zustimmten. Ferner stimmten zehn von 16 Studierenden der Aussage (eher) zu, dass das Veröffentlichen und Betrachten von Wikibeiträgen für ein gutes Gefühlt gesorgt hat. 


\begin{tabular}{|c|c|c|c|c|c|}
\hline & Stimme zu & $\begin{array}{l}\text { Stimme eher } \\
\text { zu }\end{array}$ & Teils/Teils & $\begin{array}{l}\text { Stimme eher } \\
\text { nicht zu }\end{array}$ & $\begin{array}{l}\text { Stimme nicht } \\
\text { zu }\end{array}$ \\
\hline \multicolumn{6}{|c|}{$\begin{array}{l}\text { An frei und öffentlich zugänglichen Texten (mit) zu schreiben, war für mich eine neue Erfahrung. } \\
{[N=18 ; M=1,7 ; S D=1,3]}\end{array}$} \\
\hline & $\begin{array}{l}12 \\
(66,7 \%)\end{array}$ & $\begin{array}{l}3 \\
(16,7 \%)\end{array}$ & $\begin{array}{l}1 \\
(5,6 \%)\end{array}$ & $\begin{array}{l}0 \\
(0 \%)\end{array}$ & $\begin{array}{l}2 \\
(11,1 \%)\end{array}$ \\
\hline \multicolumn{6}{|c|}{$\begin{array}{l}\text { Für mich hat es eine besondere Bedeutung gehabt, an öffentlichen Texten (mit) zu schreiben. } \\
{[N=18 ; M=3,0 ; S D=1,3]}\end{array}$} \\
\hline & $\begin{array}{l}1 \\
(5,6 \%)\end{array}$ & $\begin{array}{l}7 \\
(38,9 \%)\end{array}$ & $\begin{array}{l}5 \\
(27,8 \%)\end{array}$ & $\begin{array}{l}1 \\
(5,6 \%)\end{array}$ & $\begin{array}{l}4 \\
(22,2 \%)\end{array}$ \\
\hline \multicolumn{6}{|c|}{$\begin{array}{l}\text { Die Möglichkeit, dass Menschen außerhalb unseres Seminars meine Texte lesen können, hat } \\
\text { mein Schreiben verändert. [N =17; } M=4,1 ; S D=0,9]\end{array}$} \\
\hline & $\begin{array}{l}0 \\
(0 \%)\end{array}$ & $\begin{array}{l}0 \\
(0 \%)\end{array}$ & $\begin{array}{l}5 \\
(29,4 \%)\end{array}$ & $\begin{array}{l}5 \\
(29,4 \%)\end{array}$ & $\begin{array}{l}7 \\
(41,2 \%)\end{array}$ \\
\hline \multicolumn{6}{|c|}{$\begin{array}{l}\text { Das Veröffentlichen unserer Texte auf Wikibooks sowie das anschließende Betrachten hat für ein } \\
\text { gutes Gefühl gesorgt. [N=16; } M=2,3 ; S D=1,3]\end{array}$} \\
\hline & $\begin{array}{l}5 \\
(31,3 \%)\end{array}$ & $\begin{array}{l}5 \\
(31,3 \%)\end{array}$ & $\begin{array}{l}3 \\
(18,8 \%)\end{array}$ & $\begin{array}{l}2 \\
(12,5 \%)\end{array}$ & $\begin{array}{l}1 \\
(6,3 \%)\end{array}$ \\
\hline
\end{tabular}

Tab. 7.14: Erfahrungen der Studierenden mit Wikibooks und Bedeutung des öffentlichen Schreibens.

Mit der öffentlichen Entwicklung des Wikibookprojektes waren die Zwischenergebnisse der Studierenden und des Projektes öffentlich zugänglich, $d$. h. sowohl für die eigenen Kommilitoninnen und Kommilitonen als auch für Menschen ausserhalb des Seminars. Als Reaktion auf das gestartete Wikibookprojekt sowie auf die Zwischenergebnisse der Studierenden wurden im Verlauf der Veranstaltungen Kommentare von Menschen der Wikibook-Community formuliert (z. B. Kap. 7.2.2.3) und im Rahmen des Seminars thematisiert. Im Seminar entstand aus der Perspektive des Lehrenden mitunter der Eindruck, die Studierenden würden die Rückmeldungen der Community nicht wahrnehmen. In der Evaluation zeigt sich jedoch ein vielfältigeres Bild (Tab. 7.15). Bei allen drei Fragen reicht das Spektrum der fünfstufigen Skala von eins bis fünf. Die Mittelwerte reichen von $M=2,6$ bis $M=3,4$ und liegen jeweils in der Antwortkategorie «Teils/Teils». Ähnlich zu den Einschätzungen der öffentlichen Rückmeldungen und Kommentare zeigt sich bei der Bewertung der Texte der Kommilitoninnen und Kommilitonen ein heterogenes Bild. In beiden Fragen zu diesem Themenbereich wurde das Spektrum der fünfstufigen Skala ausgeschöpft. Die Mittelwerte reichen von $M=2,9$ bis $M=3,4$ und liegen damit ebenfalls in der Antwortkategorie «Teils/Teils». Interessant ist, dass die Häufigkeitsverteilung bei den Aussagen «Die Rückmeldungen von Menschen der Wikibook-Community haben mich nicht interessiert» und «Die Texte meiner Kommilitonen habe ich nicht gelesen» identisch sind. 


\begin{tabular}{|c|c|c|c|c|c|}
\hline & Stimme zu & $\begin{array}{l}\text { Stimme eher } \\
\text { zu }\end{array}$ & Teils/Teils & $\begin{array}{l}\text { Stimme eher } \\
\text { nicht zu }\end{array}$ & $\begin{array}{l}\text { Stimme nicht } \\
\text { zu }\end{array}$ \\
\hline \multicolumn{6}{|c|}{ Die Texte meiner Kommilitonen habe ich nicht gelesen. $[\mathrm{N}=18 ; \mathrm{M}=3,4 ; \mathrm{SD}=1,4]$} \\
\hline & $\begin{array}{l}2 \\
(11,1 \%)\end{array}$ & $\begin{array}{l}2 \\
(11,1 \%)\end{array}$ & $\begin{array}{l}6 \\
(33,3 \%)\end{array}$ & $\begin{array}{l}3 \\
(16,7 \%)\end{array}$ & $\begin{array}{l}5 \\
(27,3 \%)\end{array}$ \\
\hline \multicolumn{6}{|c|}{$\begin{array}{l}\text { Die Texte von meinen Kommilitonen haben mir eine gute Orientierung gegeben. } \\
{[N=17 ; M=2,9 ; S D=1,1]}\end{array}$} \\
\hline & $\begin{array}{l}1 \\
(5,9 \%)\end{array}$ & $\begin{array}{l}5 \\
(29,4 \%)\end{array}$ & $\begin{array}{l}8 \\
(47,1 \%)\end{array}$ & $\begin{array}{l}1 \\
(5,9 \%)\end{array}$ & $\begin{array}{l}2 \\
(11,8 \%)\end{array}$ \\
\hline \multicolumn{6}{|c|}{$\begin{array}{l}\text { Die Rückmeldungen von Menschen der Wikibook-Community haben mich nicht interessiert. } \\
{[N=18 ; M=3,4 ; S D=1,3]}\end{array}$} \\
\hline & $\begin{array}{l}2 \\
(11,1 \%)\end{array}$ & $\begin{array}{l}2 \\
(11,1 \%)\end{array}$ & $\begin{array}{l}6 \\
(33,3 \%)\end{array}$ & $\begin{array}{l}3 \\
(16,7 \%)\end{array}$ & $\begin{array}{l}5 \\
(27,8 \%)\end{array}$ \\
\hline \multicolumn{6}{|c|}{$\begin{array}{l}\text { Die Kommentare und Änderungen von Menschen der Wikibook-Community (z. B. Stephan Kulla, } \\
\text { Juetho) waren fachlich kompetent. }[N=16 ; M=2,6 ; S D=1,0]\end{array}$} \\
\hline & $\begin{array}{l}2 \\
(12,5 \%)\end{array}$ & $\begin{array}{l}6 \\
(37,5 \%)\end{array}$ & $\begin{array}{l}6 \\
(37,5 \%)\end{array}$ & $\begin{array}{l}1 \\
(6,3 \%)\end{array}$ & $\begin{array}{l}1 \\
(6,3 \%)\end{array}$ \\
\hline \multicolumn{6}{|c|}{$\begin{array}{l}\text { Die Kommentare von Menschen der Wikibook-Community (z. B. Stephan Kulla, Juetho) waren in } \\
\text { einem angemessenen Ton formuliert. }[N=17 ; M=3,0 ; S D=1,0]\end{array}$} \\
\hline & $\begin{array}{l}1 \\
(5,9 \%)\end{array}$ & $\begin{array}{l}4 \\
(23,5 \%)\end{array}$ & $\begin{array}{l}8 \\
(47,1 \%)\end{array}$ & $\begin{array}{l}3 \\
(17,6 \%)\end{array}$ & $\begin{array}{l}1 \\
(5,9 \%)\end{array}$ \\
\hline
\end{tabular}

Tab. 7.15: Interesse und Einschätzung der Studierenden an den öffentlichen Rückmeldungen und an den Texten der Mitstudierenden.

Ein weiterer Fragenkomplex widmete sich der Nutzung des Wiki-Editors (Tab. 7.16). Die Formulierung der Fragen orientierte sich an verschiedenen Interviewaussagen, welche ein arbeitsteiliges Schreiben bzw. Veröffentlichen von Texten auf Wikibooks zum Ausdruck bringen. Wie in den Interviews beschrieben, zeigt sich auch in den Antworten der Teilnehmerinnen und Teilnehmer der Evaluation, dass beispielsweise sechs Studierende der Aussage «Das Veröffentlichen der Texte im Wikibook habe ich übernommen», (eher) nicht zustimmten. Ob diesen Studierenden spezifische Erfahrungen zur Mediengestaltung zugänglich geworden sind, kann bezweifelt werden. Die zwei weiteren Fragen zur Einfachheit des Umgangs mit dem Wiki-Editor $(M=2,5)$ sowie zur angebotenen Unterstützung zur Nutzung des Wikibooks $(M=2,9)$ wurden im Mittel jeweils mit «Teils/Teils» beantwortet. Zugleich zeigen die hohen Standardabweichungen, dass die Mittelwerte kaum ein Abbild für die Schwerpunkte der Verteilung der Antworten darstellen (Kromrey 2009). Während für acht Studierende die Bedienung des Wiki-Editors (eher) einfach war, stimmten fünf Studierende dieser Aussage (eher) nicht zu. Für neun Studierende bot das Seminar (eher) genügend Unterstützung zur Nutzung von Wikibooks. Sieben Studierende stimmten dieser Aussage hingegen (eher nicht) zu. 


\begin{tabular}{|l|l|l|l|l|l|}
\hline & Stimme zu & $\begin{array}{l}\text { Stimme eher } \\
\text { zu }\end{array}$ & Teils/Teils & $\begin{array}{l}\text { Stimme eher } \\
\text { nicht zu }\end{array}$ & $\begin{array}{l}\text { Stimme nicht } \\
\text { zu }\end{array}$ \\
\hline Das Veröffentlichen der Texte im Wikibook habe ich übernommen. [N = 18; M = 3,0; SD = 1,4] \\
\hline \\
$\begin{array}{l}3 \\
(16,7 \%)\end{array}$ & $\begin{array}{l}4 \\
(22,2 \%)\end{array}$ & $\begin{array}{l}5 \\
(27,8 \%)\end{array}$ & $\begin{array}{l}2 \\
(11,1 \%)\end{array}$ & $\begin{array}{l}4 \\
(22,2 \%)\end{array}$ \\
\hline Die Bedienung des Wiki-Editors war für mich einfach. [N = 17; M = 2,5; SD = 1,4] \\
\hline \\
\begin{tabular}{|l|l|l|l|}
\hline \\
$(35,3 \%)$
\end{tabular} & $\begin{array}{l}4 \\
(11,8 \%)\end{array}$ & $\begin{array}{l}4 \\
(23,5 \%)\end{array}$ & $\begin{array}{l}1 \\
(5,9 \%)\end{array}$ \\
\hline Das Seminar bot genügend Unterstützung zur Nutzung von Wikibooks. [N = 18; M = 3,0; SD = 1,5] \\
\hline
\end{tabular}

Tab. 7.16: Einschätzung der Studierenden zur Bedienbarkeit des Wiki-Editors und Bewertung der Unterstützungsangebote.

Bei der übergreifenden Betrachtung der einzelnen Fragenkomplexe zur Bewertung der Erfahrungen zur Mitgestaltung eines Wikibooks sind verschiedene Phänomene interessant. Dazu gehört u. a., dass das Schreiben an frei und öffentlich zugänglichen Texten für Studierende eine neue Erfahrung darstellt. Die Bedeutung an entsprechenden Texten mitzuschreiben, wurde unterschiedlich eingeschätzt (Tab. 7.14). Eine hohe Streuung der Antworten zeigt sich auch in den Aussagen zum Interesse und zur Einschätzung der öffentlichen Rückmeldungen und Texte der Mitstudierenden (Tab. 7.15) sowie zur Bedienbarkeit des Wiki-Editors und zur Bewertung der Unterstützungsangebote (Tab. 7.16).

\subsection{Selbsteinschätzung des Lernerfolges}

Der letzte Fragenkomplex beinhaltet die Selbsteinschätzung der Studierenden hinsichtlich des eigenen Lernens. Damit wird ein Blick auf die - subjektiv erfahrenen - Ergebnisse des Seminars möglich (Tab. 7.17). Die eigenen Lernerfahrungen in didaktischer Hinsicht wurden eher positiv bewertet. Hingegen fühlten sich nur wenige Studierende sicherer im Umgang mit dem Wiki-Editor.

Aussagen zum fachlichen Lernen ${ }^{219}$ wurden jeweils von etwa der Hälfte der Studierenden (eher) zustimmend bewertet. Zehn von 19 Studierenden schätzen ihren fachlichen Lernzuwachs als (eher) hoch ein $(M=2,6)$. Durch das Seminar fühlten sich elf von 19 Studierenden (eher) in der Lage, strukturierter als zuvor über Lehr- und Lernsituationen nachdenken zu können $(M=2,5)$ sowie neun Studierende fühlten sich (eher) in der Lage verschiedene Lehr- und Lernsituationen pädagogisch begründet beurteilen zu können ( $M=2,8)$. Zugleich befinden sich die Mittelwerte der Antworten

219 Das fachliche Lernen umfasste hier die Entwicklung einer pädagogischen Artikulations- und Reflexionsfähigkeit (Kap. 6.4.1). Inhaltlich stand dafür u. a. die Relationierung eigener Vorstellungen zu didaktischen sowie bildungstheoretischen Themen im Fokus (Kap. 6.6.3). 
zu den drei Aussagen zwischen den Antwortkategorien «Stimme eher zu» und «Teils/ Teils». Die Aussagen zur gewonnenen Sicherheit im Umgang mit Wikis sowie zur Sinnhaftigkeit des aktiven Mitschreibens an öffentlichen Wikis umfasst ebenfalls die gesamte Breite des Antwortspektrums. Dabei sind die Studierenden im Mittel skeptisch hinsichtlich einer neu gewonnen Sicherheit. Im Mittel bewerteten die Studierenden diese Aussagen mit «Stimmer eher nicht zu» bei einem Mittelwert von $M=3,5$ und einer Standardabweichung von SD = 1,2. Die Sinnhaftigkeit des Mitschreibens an öffentlichen Wikis wurde im Mittel mit «Teils/Teils» bewertet. Die Häufigkeitsverteilung sowie die hohe Standardabweichung SD = 1,3 zeigen dabei erneut die heterogenen Einschätzungen im Kontext der Aussagen zum Wikibook.

\begin{tabular}{|l|l|l|l|l|l|}
\hline & Stimme zu & $\begin{array}{l}\text { Stimme eher } \\
\text { zu }\end{array}$ & Teils/Teils & $\begin{array}{l}\text { Stimme eher } \\
\text { nicht zu }\end{array}$ & $\begin{array}{l}\text { Stimme nicht } \\
\text { zu }\end{array}$ \\
\hline
\end{tabular}

Ich schätze meinen fachlichen Lernzuwachs (zum Thema Lehren und Lernen) durch dieses Seminar als hoch ein. $[N=19 ; M=2,6 ; S D=1,0]$

\begin{tabular}{|l|l|l|l|l|l|}
\hline & 1 & 9 & 6 & 2 & 1 \\
$(5,3 \%)$ & $(47,4 \%)$ & $(31,6 \%)$ & $(10,5 \%)$ & $(5,3 \%)$ \\
\hline
\end{tabular}

Durch das Seminar fühle ich mich in der Lage, strukturierter über Lehr- und Lernsituationen nachdenken zu können. [N=19; $M=2,5 ; S D=0,8]$

\begin{tabular}{|l|l|l|l|l|l|}
\hline & $\begin{array}{l}1 \\
(5,3 \%)\end{array}$ & $\begin{array}{l}10 \\
(52,6 \%)\end{array}$ & $\begin{array}{l}7 \\
(36,8 \%)\end{array}$ & $\begin{array}{l}0 \\
(0 \%)\end{array}$ & $\begin{array}{l}1 \\
(5,3 \%)\end{array}$ \\
\hline \\
$\begin{array}{l}\text { Durch das Seminar fühle ich mich in der Lage, verschiedene Lehr- und Lernsituationen pädago- } \\
\text { gisch begründet beurteilen zu können. [N = 19; M = 2,8; SD = 1,1] }\end{array}$ \\
\hline \begin{tabular}{l|l|l|l|l}
$\mid$ \\
$(10,5 \%)$
\end{tabular} & 7 & 4 & $\begin{array}{l}5 \\
(26,3 \%)\end{array}$ & \begin{tabular}{l}
$(5,3 \%)$ \\
\hline
\end{tabular}
\end{tabular}

Das Mitschreiben an einem Wikibook hat mir mehr Sicherheit gegebenen, mich zukünftig aktiv in Wikis einzubringen. [ $\mathrm{N}=19 ; \mathrm{M}=3,5 ; \mathrm{SD}=1,2]$

\begin{tabular}{|l|l|l|l|l|l|}
\hline & 1 & 3 & 4 & 7 & 4 \\
$(5,3 \%)$ & $(15,8 \%)$ & $(21,1 \%)$ & $(36,8 \%)$ & $(21,1 \%)$ \\
\hline
\end{tabular}

Es erscheint mir sinnvoll, mich an öffentlichen Wiki-Projekten (wie Wikibooks und Wikipeda) aktiv einzubringen. $[N=19 ; M=3,0 ; S D=1,3]$

\begin{tabular}{|l|l|l|l|l|l|}
\hline & 4 & 2 & 6 & 5 & 2 \\
& $(21,1 \%)$ & $(10,5 \%)$ & $(31,6 \%)$ & $(26,3 \%)$ & $(10,5 \%)$ \\
\hline
\end{tabular}

Tab. 7.17: Subjektive Einschätzung des eigenen Lernens durch die Studierenden.

\subsubsection{Auswertung der qualitativen Evaluationsdaten}

Um einen Einblick in die studentische Perspektive zum Erleben des Seminars zu erhalten, wurden die Studierenden im Rahmen der Abschlusssitzungen gebeten, mithilfe von Moderationskarten ihre individuellen Einschätzungen zu notieren und anschliessend in einem Gruppengespräch ( 3 bis 4 Personen) vorzustellen und zu diskutieren (Kap. 7.3.2.2). Auf Basis einer zusammenfassenden Inhaltsanalyse der aufbereiteten Gruppengespräche (Kap. B.5) konnten die Aussagen der Studierenden zu den folgenden Themen verdichtet werden. 
- Originalität und Innovation des Seminarkonzeptes (Kap. 7.4.2.2.1)

- Wikibooks als neuer Erfahrungsraum vs. «Hochladeplattform» (Kap. 7.4.2.2.2)

- Verschiebung der Inhaltserwartungen: digitale Medien im Unterricht (Kap. 7.4.2.2.3)

- Hilfreiche und nachvollziehbare Rückmeldungen (Kap. 7.4.2.2.4)

- Problematisierung der Passivität von Mitstudierenden (Kap. 7.4.2.2.5)

- Freiräume vs. Strukturierungswünsche zur Gruppenarbeit (Kap. 7.4.2.2.6)

Mit dieser Auswahl werden die zentralen Kategorien ${ }^{220}$ der zusammenfassenden Inhaltsanalyse dargestellt und die Relevanz für die Auswertung und Reproblematisierung skizziert. Zur Plausibilisierung der Kategorien werden ausgewählte Zitate der Gruppengespräche verwendet (Anhang B.5).

\subsection{Originalität und Innovation des Seminarkonzeptes}

Für beide Lehrveranstaltungen wurde die Seminargestaltung bzw. die Durchführung des Seminars positiv bewertet. Eine wiederkehrende Begründung für diese positive Bewertung war die neue Form der Seminargestaltung bzw. die Abwechslung im Vergleich zu anderen Seminarkonzepten. Dabei verwiesen die Studierenden auf Seminare, welche sich nach einer Präsentation oder dem gemeinsamen Lesen eines Textes vor allem durch eine gemeinsame Diskussionsphase charakterisieren lassen.

«Super fand ich eben [KR: keine 08/15 Durchführung des Seminars], dass es keine 08/15 Durchführung des Seminars war, so nach dem Standardverfahren der anderen Dozenten, immer dieses: ihr lest nen Text, dann ein Referat, Punkt» (D3, BLT).

Die positiven Bewertungen wurden zudem mit Bezug auf den inhaltlichen bzw. strukturellen Aufbau des Seminars begründet. Diese Begründungen umfassten eher vorsichtig formulierte Aussagen, z. B. «es war ein bisschen strukturierter und langfristiger» (B1, TC) sowie umfangreichere Formulierungen, in denen die Strukturierung des Seminars in unterschiedlichen Phasen als sinnvoll erlebt wurde.

«Meiner Meinung nach war der Aufbau gelungen [KR strukturierter Aufbau -> Phasen], dass man sich eben zuerst die Metaphern hergeleitet hat, dann eben eine Theorie sozusagen sich angeeignet hat und das dann verglichen hat. Also

220 Im Rahmen der Gruppengespräche wurden von den Studierenden auch Aspekte thematisiert, welche über die drei didaktischen Handlungsebenen «l. (Inter)Aktionen», «ll. (Lern)Situationen» sowie «III. (Lehr)Veranstaltungen» nach Wildt $(2002,8)$ hinausgehen und somit nur begrenzt von einzelnen Lehrenden mitgestaltet werden können. Dazu zählte die positive Beurteilung der «Themenwahl» (D, TC) bzw. der «theoretischen Inhalte» (B2, KU) der Seminare. Wenngleich die Themenwahl Hochschullehrenden verschiedene Freiräume und Gestaltungsentscheidungen eröffnet, ist diese auch durch die Modulbeschreibungen vorstrukturiert. Eine weitere Kategorie, die nur begrenzt durch Hochschullehrende beeinflusst werden kann, ist die Wahl eines Seminarraumes bzw. einer Seminarzeit. Zudem sind bestimmte Kategorien auf die Aussagen von Einzelpersonen zurückzuführen, diese werden daher nicht weiterverfolgt. 
von den Phasen her fand ich das gut und von der Strukturierung. Das fand ich sehr sinnvoll» (D3, OK).

Ferner wurden einzelne Elemente der Seminargestaltung positiv bewertet, u. a. die wechselnden Gruppenphasen sowie die jeweils unterschiedlichen Lehr- und Lerntätigkeiten. Weitere Aspekte und Begründungen für die positiv bewertete und als Abwechslung erlebte Seminargestaltung verwiesen auf die Medienwahl, die erhaltenen Rückmeldungen sowie die Produktorientierung ${ }^{221}$.

\subsection{Wikibooks als neuer Erfahrungsraum vs. «Hochladeplattform»}

Die Verwendung der Plattform Wikibooks sowie die Erstellung eines Wikibooks im Rahmen eines Seminars wurde von den Studierenden unterschiedlich eingeschätzt. Während Studierende den Einsatz von Wikibooks einerseits mit verschiedenen Begründungen positiv hervorhoben, markierten andere Studierende konkrete Schwierigkeiten und Wünsche zur Verwendung von Wikibooks im Rahmen eines Seminars.

Positive Bewertungen zum Einsatz von Wikibooks wurden von verschiedenen Studierenden mit dem Einsatz eines neuen Mediums begründet. Diese Begründungen knüpften einerseits an das erste Thema «[...] es war eben nicht so ein 08/15 Seminar» (Kap. 7.4.2.2.1) an und beschrieben den Einsatz von Wikibooks als "Abwechslung» (D3, BLT). Zum anderen wurde die Verwendung von Wikibooks im Seminar positiv bewertet, weil es als wichtig eingeschätzt wurde, den Umgang mit neuen Medien zu lernen und weil Wikis Einsatzmöglichkeiten in der Schule eröffnen. Ein weiteres Begründungsmuster für die positive Einschätzung umfasste die Produktorientierung bzw. die Erstellung eines (Text)Produktes sowie die damit verbundenen praktischen Tätigkeiten. Die Aussagen von Studierenden hoben zum einen die Tätigkeiten und neuen Erfahrungen hervor, z. B.: «Ich fand es gut [KR: neue Erfahrungen mit dem Text schreiben], dass wir neue Erfahrungen mit dem Text schreiben uns angeeignet haben» (D2, CP). Zum anderen wurde stärker das erstellte Produkt bzw. Werk betont, welches über Wikibooks offen zur Verfügung stand.

«Also, ich fand es gut, dass wir erst mal was Praktisches gemacht haben [KR: Praktische Arbeit, Erstellen eines Werkes] $]^{222}$. Dass wir da was erstellt haben, was man im Endeffekt dann irgendwie so in der Hand hat, was man immer wieder angucken kann und was tatsächlich sogar andere irgendwie auch bewerten» (B1, NH).

221 Die Rückmeldungen verwiesen damit in unterschiedlicher Weise auf die geplanten Lehrhandlungen zur «Produkt- und Projektorientierung» (Kap. 6.5.1), zur «Kooperation, Kollaboration und Kommunikationsorientierung» (Kap. 6.5.2) und zur «Eröffnung von Partizipationsmöglichkeiten und Erfahrungsorientierung» (Kap. 6.5.3) sowie auf Entscheidungen zum Entwurf des konkreten Seminarkonzeptes (Kap. 6.6.2).

222 Im Zusammenhang mit der getroffenen Aussage platzierte die Person eine rote Moderationskarte mit den Stichpunkten: «Praktische Arbeit, Erstellen eines Werkes». 
Die Bewertung durch Andere kann in diesem Zitat als Verweis auf die Rückmeldungen durch die Wikibook-Community interpretiert werden. Die Bewertung und Rückmeldung durch die Wikibook-Community erfolgte bereits im Verlauf des Seminars vor allem durch die zwei Wikibookianer «Juetho» und "Stephan Kulla» (Kap. 7.2.2.3, 7.2.3.3, 7.2.4.3). In einer Diskussionsgruppe wurden diese Rückmeldungen auf unterschiedlichen Ebenen thematisiert und unterschiedlich bewertet. Eine Person hat die Rückmeldungen durch Juetho als eher abwertend kommentiert, wie sich in umgangssprachlichen Formulierungen zeigte, z. B. «Jürgen hat halt einfach abgekotzt und das wars, ne» (D2, FC). Zugleich wurde in der Diskussion auch die Unsicherheit zum Ausdruck gebracht, wie Rückmeldungen von Menschen im Kontext einer offenen Sharing-Gemeinschaft eingeschätzt werden können.

«FC: Beziehungsweise Jürgen hat ja auch über pädagogische Sachen abgekotzt, (TT: Ja, genau.), aber man war sich da nicht so sicher ob er das beurteilen kann.

TT: Genau und ob er da so das Fachwissen und die Kompetenz dahinter hat, deswegen fand ich das so ein bisschen unangebracht» (Gruppe D2).

Wenngleich die Kommentare von «Juetho» in dieser Gruppe einerseits problematisiert wurden, wurde andererseits auch der Wunsch nach mehr Rückmeldungen von «anderen Fachpersonen» (D2, TT) zum Ausdruck gebracht.

«In Bezug auf Jürgen, ich hab jetzt mal geschrieben [KG: Rückmeldung zu den verfassten Texten von weiteren Fachpersonen, die sich auch mit der Erstellung von Wikis auskennen], dass es für mich irgendwie noch gefehlt hat, dass es keine Rückmeldung von anderen Fachpersonen gab. Also Jürgen hat zwar eine Rückmeldung zu unserem Schreibstil auf Wikibooks gegeben, aber er hat eigentlich kein wirklich fachliches Wissen über Pädagogik und dementsprechend hat er quasi formale Fehler korrigiert und inhaltliche Fehler wurden zwar von Franco und Sophie korrigiert, aber ich hätte mir gewünscht, dass es vielleicht noch eine Person gibt, die in beiden Aspekten sehr gut sich auskennt» (D2, TT).

Neben dem Wunsch nach «anderen Fachpersonen» (D2, TT) wurden in dieser Diskussionsgruppe weitere Wünsche zur Verwendung des Wikibooks formuliert sowie verschiedene Aspekte problematisiert. Die Wünsche umfassten u. a. motivationale Aspekte. Beispielsweise wurde der Wunsch formuliert, dass mehr Interesse für die Beteiligung am Wikibook geweckt werden sollte und eine Begründung für die Nutzung von Wikibooks wünschenswert gewesen wäre. Problematisiert wurde die Einbindung von Wikibooks in das Seminar, welche teilweise nur als Plattform zum Hochladen von Texten erlebt wurde. 
«Ja und für mich war auch noch ein Flop [KB: Wikibook wie 2. Moodle], dass das Wikibooks so ein grosses Thema war eigentlich, aber dann letzten Endes eigentlich nur als 2. Moodle genutzt wurde, wo die Texte hochgeladen werden und ich weiss zwar nicht genau wie, aber ich denke, da hätte man noch bisschen draus machen können. Dass auch die Motivation, mit dem Wikibook zu arbeiten bisschen steigt. Ausser nur mit Jürgen zu (kurze Pause), naja nicht mal zu diskutieren, aber zu lesen» (D2, KC).

Weitere Kritikpunkte an der Einbindung des Wikis wurden vereinzelt auch in anderen Gruppen formuliert. Diese bezogen sich auf die Problematisierung fehlender Unterstützung hinsichtlich der Bedienung des Wiki-Editors sowie den Wunsch nach mehr Wissen über die Funktionsweise von Wikibooks.

\subsection{Verschiebung der Inhaltserwartungen: digitale Medien im Unterricht}

Ein weiteres Thema, das nur von Lehramtsstudierenden formuliert wurde, bezieht sich insbesondere auf den Einsatz von neuen Medien im Unterricht. So brachten verschiedene Studierende seminarübergreifend zum Ausdruck, dass innen der Bezug zur «Praxis im Unterricht» sowie der «Anwendungsbezug in der Schule» (D1, BK) fehlte. Da beide Veranstaltungen hinsichtlich des Modulhandbuchs in der Bildungstheorie sowie als Seminar für die Allgemeine Didaktik verortet waren, war das Interesse an einer expliziten Auseinandersetzung mit neuen Medien überraschend. Dabei wurde in den Aussagen der Studierenden auf neue Medien im Allgemeinen sowie explizit auf das Wikibook Bezug genommen.

«Was mir ein bisschen gefehlt hat, [KG: Umgang neuer Medien im Unterricht] ist, dass man den Umgang von den neuen Medien noch mal reflektiert, auch wie man das im Unterricht einsetzen kann, das kam mir hier ein bisschen zu kurz. Vielleicht dass man einfach noch mal darüber geredet hätte, wie man das Wikibook, ob man das im Unterricht einsetzen kann, ob die Form gut ist oder auch nicht» (D3, DL).

Zudem wurde von verschiedenen Studierenden darauf hingewiesen, dass auch weitere neue Medien im Seminar diskutiert hätten werden können. Offen bleiben muss an dieser Stelle die Frage, ob das Seminar das Interesse an diesen Themen erst geweckt hat bzw. sich die Studierenden bereits vor dem Seminar für diese Fragen interessierten. 


\subsection{Hilfreiche und nachvollziehbare Rückmeldungen}

Die von uns schriftlich formulierten Rückmeldungen zu den verschiedenen Textprodukten wurden von den Studierenden thematisiert und durchgehend wertschätzend beurteilt. Begründet wurden die positiven Bewertungen der Rückmeldungen hinsichtlich (a) des Umfangs und der Verständlichkeit, (b) der individuellen Formulierung zu den jeweils konkreten Textprodukten sowie (c) hinsichtlich der hilfreichen Hinweise zur Überarbeitung. In den Äusserungen wurden teilweise einzelne Facetten betont, z. B. «und was ich auch noch sehr gut fand [KR: hilfreiche Rückmeldungen], waren die hilfreichen Rückmeldungen, wo wir sozusagen auch noch überarbeiten können» (D2, CP). In anderen Aussagen der Studierenden wurden mehrere Aspekte zum Ausdruck gebracht:

«Dann fange ich mal mit den Tops an und zwar waren wir uns relativ einig und zwar die Rückmeldung zu unseren Texten und Ausarbeitungen war sehr umfangreich und verständlich [KR: Gute Rückmeldungen zu den Texten]. Ausserdem waren die Seminarleiter sehr engagiert, dass auch sie uns ordentliches Feedback geben. [KR: Zuständige für das Seminar waren sehr engagiert -> jeder Text wurde individuell bewertet etc.]» (D1, BK).

Neben der Wertschätzung der Rückmeldungen wurden von dieser Gruppe auch das Engagement der Lehrenden hervorgehoben. Weitere Aussagen zur Aktivität der Lehrenden hinsichtlich der Formulierungen von Rückmeldungen wurden u. a. in Abgrenzung zur Aktivität der Studierenden formuliert. In der Beschreibung einer Nachfrage im Moodle-Forum wurde beispielsweise die Aussage formuliert «von Franco und Sophie kam was, das ist aber deren Job, aber von den Teilnehmern kam von wenigen überhaupt Feedback» (B1, TC). Die Formulierung «das ist aber deren Job» erscheint eher als Ausdruck dafür, dass eine Rückmeldung auch erwartet wird.

\subsection{Problematisierung der Passivität von Mitstudierenden}

Ausbleibende Rückmeldungen von Mitstudierenden sind ein Beispiel für die Passivität, welche von verschiedenen Studierenden im Rahmen der Evaluationssitzung problematisiert wurde. Diese Kritik an der Passivität von Mitstudierenden bezog sich auf die Beteiligung am und im Seminar im Allgemeinen sowie auf die Beteiligung in konkreten Gruppenarbeiten und Projektphasen. Die Formulierung der ausbleibenden Beteiligung am und im Seminar wurde von Studierenden hinsichtlich der geringen Anwesenheit in den Präsenzseminaren sowie deren Zurückhaltung in Diskussionen zum Ausdruck gebracht.

"Was mir, was mich gestört hat in dem Seminar war [KB: mangelnde Beteiligung], dass oftmals sehr wenige da waren und auch immer die gleichen, obwohl es mehr Leute gibt, die an dem Seminar teilnehmen und auch die Leute, 
die da waren, haben sich zum Teil komplett zurückgezogen und gefühlt zumindest in einer Sitzung gar nichts gesagt. Ich erinnere mich z. B. an meine Sitzung, wo ich meinen Text vorgestellt hab, da waren insgesamt fünf Leute da und davon hatten zwei den Text geschrieben und drei weitere, und davon haben ein oder zwei Leute gar nichts gesagt, das war dann irgendwie ein bisschen schwierig» (B1, TC).

Darüber hinaus wurde der Wunsch nach mehr Beteiligung auf Moodle in Form von gruppenübergreifendem Peer-Feedback formuliert. Zugleich wurden bereits in der Zusammenarbeit der Gruppen verschiedene Probleme hinsichtlich der Aktivität der Gruppenmitglieder zum Ausdruck gebracht. Unabhängig von der Gruppenphase wurde von verschiedenen Studierenden die Zusammenarbeit mit ihren Gruppenmitgliedern problematisiert.

«Dann unsere Flops waren, [KB: Kommunikation innerhalb der Gruppen tw. schwierig, Startschwierigkeit] [...]. Ausserdem [KB: Gruppenzusammenarbeit in der 1. Erarbeitungsphase] gibt es innerhalb der Gruppen auch immer die Leute, die was machen und die Leute, die nichts machen. Wie man das aus der Schule kennt. Das hatten wir halt auch teilweise» (D1, BK).

«Weniger gut gefallen hat mir [...] die Absprache in unserer Gruppe während der zweiten Projektphase und die teilweise fragwürdige Motivation meiner Kommilitonen» (B2, KU).

Zudem wurden die Probleme der Gruppenarbeit von weiteren Studierenden mit der Überforderung einiger Gruppenmitglieder begründet. In diesem Zusammenhang wurde von Studierenden der Aufwand für die zu erbringende Studienleistung als hoch eingeschätzt.

\subsection{Freiräume vs. Strukturierungswünsche zur Gruppenarbeit}

Im Zusammenhang mit der Durchführung von Gruppenarbeiten wurde von den Studierenden Aussagen zu einem weiteren Thema getroffen. Dieses bewegt sich zwischen den positiv bewerteten Freiräumen zur Selbst- und Gruppenorganisation einerseits sowie dem Wunsch nach bzw. der Kritik an fehlenden Orientierungshilfen andererseits. Der Wunsch nach Orientierungshilfen drückt sich zum einen am Fehlen formaler (und inhaltlicher) Orientierungsangebote aus. Zum anderen wird das Verhältnis zwischen eigenständiger Gruppenarbeit und (fehlender) gemeinsamer Präsenzveranstaltungen problematisiert. 
Verschiedene Studierende wertschätzten die im Seminar eröffneten Freiheiten zur Selbst- und Gruppenorganisation. Insbesondere die Möglichkeit, einen eigenen Zeitplan im Rahmen der Seminarphasen für sich selbst gestalten zu können, wurde positiv bewertet.

«Gut fand ich [KR: Selbst-/Gruppenorganisation], die Selbst-/Gruppenorganisation, dass wir uns selbst einteilen konnten und unseren eigenen Zeitplan im Endeffekt gestalten konnten» (B2, BH).

In diesem Zusammenhang eröffnete das Seminarkonzept mit Schreibzeiten ohne Präsenztermine Freiheiten für Studierende, welche entsprechend positiv bewertet wurden. "Gerade wenn auch, gerade weil viel Zuhausearbeit anfiel, die Stunden auch eben ausgefallen sind» (D1, BK). Der «Ausfall von Stunden», wie es die Studierenden bezeichneten sowie der Umstand, dass es keine Anwesenheitspflicht gab, wurde auch von weiteren Studierenden positiv hervorgehoben. Zugleich wurde das Verhältnis zwischen selbstorganisierten Schreibphasen und gemeinsamen Präsenzterminen von verschiedenen Studierenden problematisiert, da so u. a. die Präsenzzeit als «zerstückelt» (B2, KU) erlebt wurde. Andere Aussagen lassen sich dahingehend deuten, dass die Schreibzeiten nicht als Freiraum zur Selbst- und Gruppenorganisation erlebt wurden. Vielmehr entstand durch die Möglichkeit, sich selbst zu organisieren, eine Überforderung und es wurde der Wunsch geäussert, im Rahmen der Präsenztermine in den Gruppen zusammenarbeiten zu können.

«Was mir einfach gefehlt hat [KG: viel Heimarbeit statt im Seminar], ist, dass wir hier im Seminar sehr wenig dafür gearbeitet haben, und viel Heimarbeit hatten. Das war echt manchmal überfordernd, weil wir ja auch andere Veranstaltungen haben und ich würde mir wünschen, dass wir vielleicht zwei-, dreimal mehr hier Seminar mit den Gruppen zusammengesessen hätten» (D2, CP).

Der Wunsch nach bzw. die Kritik an fehlenden formalen Orientierungs- und Strukturierungshilfen stand ebenfalls in Spannung zu den positiv bewerteten Freiräumen. Ausserdem wurde von vereinzelten Studierenden problematisiert, dass die Erstellung der Gruppenmetapher zu Beginn als schwierig erlebt wurde, da es keine Vorlage gab. Eine Person formulierte beispielsweise den Vorschlag, bereits zu Beginn der verschiedenen Seminarphasen die verwendeten Feedbackbögen zur Verfügung zu stellen: «also mit den Richtwerten, an was man sich halten soll» (B1, NS). Zugleich formulierte eine andere Person: «Es waren eindeutige Kriterien zum Bestehen der Studienleistung da» (B2, LI). Der grösste Kontrast zur positiven Bewertung der eröffneten Freiräume stellte die Kritik am nicht als verpflichtend erlebten Orientierungsrahmen des Seminars dar: 
«Und was mich auch ein bisschen gestört hat [KB: keine Verpflichtungen (Abgabefristen, Anwesenheit)], auch wenn ich eigentlich kein Fan von Anwesenheitspflicht oder irgendwas Ähnlichem bin, wenn halt gesagt wird, die Deadline ist am, und im nächsten Satz kommt dann, naja, wer es bis dahin nicht fertig hat, ist auch nicht schlimm. Das ist dann irgendwie so sehr unverbindlich und das verleitet dann bei vielen dazu, dass sie es dann entweder gar nicht oder sehr spät machen, das fand ich auch ein bisschen schwierig» (D3, TC).

Neben dem Wunsch nach formalen Orientierungshinweisen fanden sich in einer Diskussionsgruppe des Seminars «Bildung metaphorisch verstehen» auch Wünsche und Verbesserungshinweise zur Bereitstellung von Orientierungshilfen zur Erarbeitung von Bildungstheorien.

\subsubsection{Zusammenfassung und Relevanz der Evaluationsergebnisse}

Mit der Durchführung der Veranstaltungsevaluation und der Auswertung der quantitativen sowie der qualitativen Daten (Kap. 7.4.2.1, 7.4.2.2) wurde das Ziel verfolgt, insbesondere Erkenntnisse zu zwei zentralen Fragestellungen zu gewinnen. Diese Fragestellungen fokussieren die Erfahrungsmöglichkeiten zum Lernen mit sozialen Medien (Kap. 3.2.1.3) einerseits und potenzielle Lernsituationen zur Teilhabe an öffentlichen Wikis (Kap. 3.2.1.2) anderseits. Die zentralen Ergebnisse der quantitativen Veranstaltungsevaluation umfassen studentische Positionen zur Einschätzung der Lehrveranstaltung (Kap. 7.4.2.1.1), zur Bewertung der Seminaranforderungen und Lehr-Lerninteraktionen (Kap. 7.4.2.1.2), zur Bewertung der Erfahrungen zur Mitgestaltung eines Wikibooks (Kap. 7.4.2.1.3), sowie zur Selbsteinschätzung des Lernerfolges (Kap. 7.4.2.1.4). Dabei wurde das gesamte Seminar als «gut» bewertet und die Seminarstruktur von den Studierenden positiv eingeschätzt (Kap. 7.4.2.1.1). Problematisiert wurden u. a. Aussagen zur Aktivität der Gruppe sowie zur Gruppenbildung (Kap. 7.4.2.1.2). Die Durchführung des Wikibookprojektes ermöglichte der Mehrheit der Studierenden neuen Erfahrungen bei der Auseinandersetzung mit digitalen Medien. Zugleich waren die Einschätzungen und Bewertungen der Erfahrungen zur Nutzung des Wikibooks sowie zur Wahrnehmung der Wikibook-Community sehr unterschiedlich (Kap. 7.4.2.1.2).

Mithilfe einer Inhaltsanalyse konnten die qualitativen Aussagen der Studierenden zu sechs zentralen Themen zusammengefasst werden. Hinsichtlich des Konzeptes zum Lernen mit digitalen Medien (Kap. 3.2.1.3) sind insbesondere die Aussagen zur Originalität und Innovation des Seminarkonzeptes (Kap. 7.4.2.2.1), zu Inhaltserwartungen zur Thematisierung digitaler Medien (Kap. 7.4.2.2.3) sowie zu den als hilfreich und nachvollziehbar bewerteten Rückmeldungen (Kap. 7.4.2.2.4) relevant. Erkenntnisse zur Frage nach potenziellen Anlässen zum Lernen über soziale Medien 
(Kap. 3.2.1.2) bietet das kontrovers diskutierte Thema «Wikibooks als neuer Erfahrungsraum vs. 〈Hochladeplattform» (Kap. 7.4.2.2.2). Die zwei weiteren Themen zur «Problematisierung der Passivität von Mitstudierenden» (Kap. 7.4.2.2.5) sowie zum «Verhältnis von Freiräumen vs. Strukturierungswünschen zur Gruppenarbeit» (Kap. 7.4.2.2.6) sind anknüpfungsfähig an die geäusserte Kritik im quantitativen Fragebogen (Kap. 7.4.2.1).

Relevante Aspekte werden im folgenden Kapitel «Auswertung und Neuperspektivierung» (Kap. 8) aufgegriffen und zur Modifikation des Entwurfs diskutiert. Bei vergleichender Betrachtung mit den Ergebnissen der Interviews (Kap. 7.4.1) sowie der folgenden Auswertung der Versionsgeschichte (Kap. 7.4.4) werden die Ergebnisse der Veranstaltungsevaluation zur Modifikation ausgewählter Lernsituationen (Kap. 9.2) sowie zur Modifikation der Prozessplanung (Kap. 9.3) diskutiert.

\subsubsection{Ergebnisse der Metaphernanalysen}

Mit den Ergebnissen der Metaphernanalysen wird ein systematischer Einblick in die studentischen Seminarprodukte zur Artikulation und Reflexion eigener Vorstellungen eröffnet. Durch die Formulierung, Diskussion und Reflexion von Metaphern sollten Lernsituationen geschaffen werden, um die Entwicklung einer Artikulations- und Reflexionsfähigkeit zu ermöglichen (Kap. 6.4.1, 6.6.1). Um den Anregungsgehalt dieser gestalteten Lernsituationen empirisch erfassen zu können, wurden die im Sommersemester 2015 entstandenen studentischen Arbeitsergebnisse in Form von individuell formulierten Ad-hoc-Metaphern und gemeinsamen entwickelten Gruppenmetaphern analysiert (Kap. 7.3.3). Zur Strukturierung der Ergebnisse erfolgt für die jeweiligen Arbeitsprodukte im ersten Schritt eine formale Beschreibung spezifischer Ausdrucksformen und -phänomene und im zweiten Schritt eine Rekonstruktion metaphorischer Muster:

- Ausdrucksformen und -phänomene der Ad-hoc-Metaphern (Kap. 7.4.3.1)

- Sprachfiguren und metaphorische Muster der Ad-hoc-Metaphern (Kap. 7.4.3.2)

- Darstellungsformen und Strukturelemente der Gruppenmetaphern (Kap. 7.4.3.3)

- Sprachfiguren und metaphorische Muster der Gruppenmetaphern (Kap. 7.4.3.4)

Die Darstellung der Ergebnisse der Metaphernanalyse erfolgt in Anlehnung an de Guerrero und Villamil (2002). Zur Darstellung der metaphorischen Muster werden GROSSBUCHSTABEN verwendet. Die Angabe expliziter metaphorischer Ausdrücke und Redewendungen erfolgt in Form von fettgedrucktem Text. Mit Kursivschrift werden implizite Metaphern hervorgehoben (Kap. 3.4.3). Zur Plausibilisierung der Ergebnisse werden die studentischen Seminarprodukte weitgehend im Original zitiert. 
Diese Zitate beinhalten teilweise Rechtschreibfehler ${ }^{223}$ und verwenden überwiegend das generische Maskulinum. Um einen authentischen Einblick in die studentischen Produkte zu ermöglichen, ist die Verwendung einer geschlechtergerechten Sprache in diesem Kapitel nicht gegeben. Dieses Phänomen wird in der folgenden Auswertung aufgegriffen und diskutiert (Kap. 8.2.2.3).

\subsubsection{Ausdrucksformen und-phänomene der Ad-hoc-Metaphern}

Zur Bearbeitung der Frage, «Welche Vorstellung haben Sie vom Lehren und Lernen im Unterricht?», wurden die Studierenden aufgefordert, eine Metapher zu formulieren und zu erläutern. Die in diesem Kontext erstellten studentischen Schreibprodukte lassen sich hinsichtlich unterschiedlicher Ausdrucksformen und sprachlicher Phänomene unterscheiden. Um einen strukturierten Einblick über die Ausdrucksformen und -phänomene der studentischen Schreibprodukte zu ermöglichen, werden im Folgenden drei formal unterscheidbare Ausdrucksformen skizziert, die Studierende zur Erläuterung ihrer formulierten Ad-hoc-Metapher gewählt haben.

- Explizite Benennung der übertragenden Elemente (Kap. 7.4.3.1.1)

- Exemplarische Narration/Beschreibung zur Veranschaulichung (Kap. 7.4.3.1.2)

- Mischformen von expliziten Übertragungen und exemplarischen Narrationen (Kap. 7.4.3.1.3)

Die drei benannten Formen bringen metaphorische Bedeutungsübertragungen in jeweils unterschiedlicher Weise zum Ausdruck. In Anlehnung an Schmitt (2017) werden diese Bedeutungsübertragungen jeweils als metaphorische Redewendungen und Einzelmetaphern verstanden (Kap. 5.3.3224). Am Beispiel der studentischen Schreib- und Textprodukte wird dabei auf die jeweiligen Besonderheiten des Materials aufmerksam gemacht. Als Besonderheiten des Materials werden zudem drei Aspekte einzeln hervorgehoben:

- Routinisierte Metaphern «fremder» Bildbereiche (Kap. 7.4.3.1.4)

- Bildbrüche innerhalb des Bildbereichs einer Ad-hoc-Metapher (Kap. 7.4.3.1.5)

- Nicht nachvollziehbare Bedeutungsübertragungen (Kap. 7.4.3.1.6)

Die exemplarisch skizzierten Besonderheiten wurden ausgewählt, um damit verbundene Herausforderungen auf praktischer Ebene in der Lehre sowie auf forschungsmethodischer Ebene zur Auswertung der Produkte veranschaulichen zu

223 Die Lesbarkeit der Zitate wurde durch die Kennzeichnung der Rechtschreibfehler mit der Formulierung «(sic)» stärker beeinträchtigt als durch die Rechtschreibfehler selbst. Daher wurde auf die Kennzeichnung weitgehend verzichtet.

224 Wie im Kapitel 5.3.3 dargestellt, geht der Metaphernbegriff über ein Alltagsverständnis von Metaphern hinaus. 
können ${ }^{225}$. Die Relevanz der formalen Betrachtung der studentischen Arbeitsprodukte wird im Rahmen der einzelnen Kapitel skizziert.

\subsection{Explizite Benennung der übertragenden Elemente}

Eine Form zur Erläuterung formulierter Metaphern kann als explizite Benennung bzw. Darstellung der übertragenden Elemente bezeichnet werden. Neben der Vervollständigung des Satzes «Lehren und Lernen im Unterricht ist wie ...» formulierten die Studierenden die Bedeutungsübertragung für ausgewählte Elemente bzw. stellten diese überblicksartig in Stichpunkten dar. Die folgende Formulierung steht beispielhaft für diese Darstellungsform:

"Lehren und Lernen im Unterricht ist wie wenn ein Reiseführer seine buntgemischte Reisegruppe auf holprigen Wegen durch den Nebel führt. Bis alle wieder klar sehen können und die Wege wieder eben sind:

- Reiseführer: Lehrer (Lehrt nach dem Plan),

- buntgemischte Reisegruppe: Unterschiedl. Schüler (vorlaut, hochbegabt, Lese-Rechtschreibschwäche, ADHS),

- Reise: Lernprozess,

- Nebel: Lernstoff (Am Anfang noch unverstanden),

- holpriger Weg: Probleme, die beim Lernprozess auftreten können» (D15-19).

Die vorgestellte Ad-hoc-Metapher beinhaltet vielfältige Elemente, die vom Bildbereich des Reiseführers mit seiner Reisegruppe auf den Zielbereich des Lehrens und Lernens im Unterricht übertragen wurden. Die in diesem Beispiel ausgewählten Elemente umfassen überwiegend Substantive, die in der Ad-hoc-Metapher verwendet wurden. Dargelegt wird die Bedeutung der handelnden Menschen (Reiseführer, Reisegruppe) in Auseinandersetzung mit Rahmenbedingungen (Nebel, holpriger Weg). Die Bedeutungsübertragung «Reise: Lernprozess» stellt eine Ausnahme dar. Verben bzw. die Handlungen der jeweiligen Personen werden in diesem Beispiel nicht explizit erläutert.

\subsection{Exemplarische Narration/Beschreibung zur Veranschaulichung}

Eine zweite Form zur Erläuterung der entwickelten Ad-hoc-Metaphern erfolgte in Form von «exemplarischen Narration» (Schmitt 2017, 477) bzw. «szenischen Metaphern» (Bohnsack 2010, 67). Schmitt $(2017,477)$ und Bohnsack $(2010,67)$ verstehen die Begriffe als «Beschreibungen und Erzählungen von (relativ) hohem Detaillierungsgrad, in denen zentrale Orientierungen ihren metaphorischen Ausdruck finden»

225 Eine systematische Übersicht über die formale Einschätzung aller formulierten Ad-hoc Metaphern findet sich im Anhang (Abb. B.3, B.4). 
(Bohnsack 2010, 67). In Abgrenzung von expliziten Vergleichen und begrifflichen Metaphern (Bohnsack 2010, 67) lassen sich die Erläuterungen der Studierenden als Beschreibung verstehen, um die Metaphorik ihres formulierten Vergleiches zu veranschaulichen bzw. auszuschmücken. Dabei muss diese Beschreibung selbst keine Metaphorik beinhalten. Ein Beispiel für diese Darstellungsform bietet die folgende Ad-hoc-Metapher sowie die zugehörige Erläuterung:

«Lehren und Lernen im Unterricht ist wie Ein Papierflugzeug / Ein Drachen (D15-17):

- Es kann viel oder wenig Planung im Bau stecken.

- Das Material des Papierflugzeugs ist die Grundlage.

- Wie gut er fliegen kann ist ungewiss.

- Die Art des Werfenden und er selbst beeinflussen den Flug.

- Er kann trotz seines gegebenen Materials viele Farben haben.

- Sein Umfeld beeinflusst seinen Flug» (D15-17).

Die in der Erläuterung getroffenen Aussagen zum Papierflugzeug stellen keine bzw. kaum explizit ausformulierten Bedeutungsübertragungen dar. Die gesamte Beschreibung kann jedoch als Veranschaulichung bzw. "Bebilderung» (Schmitt 2017, 477) der entworfenen Ad-hoc-Metapher, «Lehren und Lernen im Unterricht ist wie Ein Papierflugzeug», verstanden werden. In diesem Beispiel bleibt offen, wofür das Pronomen «er» sowie das zugehörige Possessivpronomen «seines» steht.

\subsection{Mischformen von expliziten Übertragungen und exemplarischen Narrationen}

Eine dritte Form zur Erläuterung von Ad-hoc-Metaphern kann als Mischform von expliziten Übertragungen und exemplarischen Narrationen bezeichnet werden. Die Vervollständigung des Satzes «Lehren und Lernen im Unterricht ist wie ...» beschränkt sich in dieser Form häufig auf wenige Worte. Dafür erfolgt eine ausführliche Veranschaulichung und Konkretisierung der Ad-hoc-Metapher in Form einer exemplarischen Beschreibung. Die Bedeutungsübertragung der relevanten Elemente wird für eine beschränkte Auswahl expliziert. Exemplarisch für diese Form der Erläuterung steht die folgende Ad-hoc-Metapher:

«Lehren und Lernen im Unterricht ist wie Gartenarbeit.

- Lehrer als Gärtner,

- Schüler als Pflanze,

- Gärtner pflanzt, pflegt,

- Gießen als Input, Gärtner kann gewisse Rahmenbedingungen festlegen, kann letztendlich nicht über Qualität des Endproduktes entscheiden» (D15-22). 
In dieser Erläuterung wird zunächst die Bedeutungsübertragung zwischen dem Zielbereich («Lehrer» und «Schüler») und dem Bild- bzw. Quellbereich der Gartenarbeit («Gärtner» und «Pflanzen») explizit formuliert. In Form einer exemplarischen Beschreibung werden im Folgenden die Tätigkeiten des «Gärtners» im Bildbereich konkretisiert. Während die Tätigkeiten des Pflanzens und Pflegens nicht offen gelegt werden, kann die Formulierung «Gießen als Input» als weitere dargelegte Bedeutungsübertragung verstanden werden. Die im Rahmen der kurzen Narration verwendeten Elemente des Bildbereichs, wie z. B. das Pflanzen durch den Gärtner, die Rahmenbedingungen des Gartens sowie die Endprodukte, werden nicht explizit übertragen. Insofern kann diese Ad-hoc-Metapher als Mischform von expliziten Übertragungen und einer exemplarischen Narration beschrieben werden.

\subsection{Routinisierte Metaphern fremder Bildbereiche}

Ein spezifisches Phänomen, welches sich in den zuvor beschriebenen Beispielen bereits identifizieren lässt, ist die Verwendung von routinisierten bzw. impliziten Metaphern (Kap. 5.3.3) zur Darstellung sowie zur Erläuterung von Ad-hoc-Metaphern. Das folgende Beispiel kann dieses Phänomen veranschaulichen:

«Lehren und Lernen im Unterricht ist wie Gärtnern/ein Gärtner bei der Gartenarbeit:

Der Gärtner gibt seinen Pflanzen alles mit auf den Weg um sich gut entwickeln und wachsen zu können. Der Lehrer vermittelt den Schülern Wissen und auch soziale Normen, die sie (teils) für das spätere Leben brauchen -> um zu wachsen / ‘aufzublühen`/ sich gut zu entwickeln» (D15-01)

In der Ad-hoc-Metapher erfolgt eine Übertragung vom Bildbereich des «Gärtnern/ ein Gärtner bei der Gartenarbeit» auf den Zielbereich Lehren und Lernen im Unterricht. In der folgenden exemplarischen Narration wird die Handlung des Gärtners mit routinisierten metaphorischen Redewendungen jedoch aus weiteren Bildbereichen veranschaulicht. Erkennbar wird dies an der Formulierung «Mitgebens» bzw. «mit auf den Weg [geben]». Da sich Pflanzen in der Regel nicht zur eigenen Entwicklung bzw. zum Wachstum fortbewegen müssen, ist die Formulierung «mit auf den Weg [geben]» an dieser Stelle nur metaphorisch zu verstehen. Wenngleich diese Metaphorik in unserem alltäglichen Sprachgebrauch routinisiert erfolgt, kann die Verwendung einer Wegmetaphorik für Pflanzen überraschen (z. B. aufgrund ihrer Wurzeln). 


\subsection{Bildbrüche innerhalb des Bildbereichs einer Ad-hoc-Metapher}

Ad-hoc-Metaphern, in denen die Erläuterung widersprüchliche Bedeutungsübertragungen und Beschreibungen aufweisen, werden im Folgenden metaphorisch unter dem Begriff «Bildbruch» zusammengefasst. Widersprüchlichkeit in einer Ad-hoc-Metapher ist dann gegeben, wenn keine kohärente bzw. zusammenhänge Metaphorik verwendet wird bzw. den übertragenden Elementen mehrere Bedeutungen zugeschrieben werden, die sich widersprechen. Exemplarisch kann dies an der folgenden Ad-hoc-Metapher skizziert werden:

«Lehren und Lernen im Unterricht ist wie ein Fenster:

Wenn die Lernenden das Fenster offenlassen, bekommen sie vieles für die Zukunft mit. Natürlich muss der Lehrende dies erlauben (Kein Schloss am Fenster)» (D15-06).

Zunächst werden die zwei Aktivitäten Lehren und Lernen im Kontext der Institution Schule mit einem «Fenster» verglichen. In der folgenden Erläuterung wird in jeweils unterschiedlicher Form auf dieses Fenster Bezug genommen. Lernenden wird die Handlungs- bzw. Entscheidungsmöglichkeit zugeschrieben, dass sie das «Fenster offenlassen». Zugleich ist damit eine Bedingung formuliert, die, wenn sie erfüllt ist, die Konsequenz hat, dass die Lernenden «vieles für die Zukunft mit[bekommen]» (D15-06). In dieser Formulierung eröffnet sich die Lesart, dass offene Fenster als offene Haltung oder Einstellung der Lernenden zu verstehen sind, beispielsweise gegenüber neuen Themen und Fächern. Der zweite Satz der Erläuterung widerspricht dieser Lesart jedoch, da der Lehrende nicht als derjenige beschrieben wird, der etwas durch das Fenster gibt, sondern als derjenige, der die Offenheit des Fensters erlauben und verhindern kann. Dieser Widerspruch lässt sich als Bildbruch deuten.

\subsection{Nicht nachvollziehbare Bedeutungsübertragungen}

Mit der Formulierung nicht nachvollziehbarer Bedeutungsübertragungen ist gemeint, dass die Erläuterung der Ad-hoc-Metapher nicht dazu beigetragen hat, diese besser zu verstehen. Exemplarisch kann dies an der folgenden Ad-hoc-Metapher skizziert werden:

«Lehren und Lernen im Unterricht ist wie Tag und Nacht:

Es gibt Themen, die vermittelt werden sollen, aber der Lernerfolg kann individuell ausfallen es gibt unterschiedliche Ergebnisse» (D15-11).

Die formulierte Erläuterung beschränkt sich auf Darstellungen im Zielbereich des Lehrens und Lernens. Bezüge zum Tag oder zur Nacht, wie die Ad-hoc-Metapher es erwarten lässt, erfolgen weder durch explizite Übertragungen noch durch routinisierte Metaphern. Entsprechende Darstellungen werden unter dem Begriff der nicht 
identifizierbaren Bedeutungsübertragung zusammengefasst. So sind verschiedene Lesarten der Ad-hoc-Metapher denkbar, aber auf Basis der Erläuterung nicht belegbar. Denkbar ist unter anderem, dass Tag und Nacht als Gegensätze zu verstehen sind und Lehren und Lernen entsprechend Gegensätze darstellen. Die Aussagen über Lehren und Lernen sind aber nicht notwendigerweise als Gegensätze zu verstehen. Wenngleich Lernerfolge individuell ausfallen können, müssen sie nicht das Gegenteil der vorgegebenen Themen darstellen. Denkbar wäre ebenfalls, dass Tag und Nacht als zyklisch aufeinander folgende Phasen verstanden werden können und entsprechend Phasen des Lehrens und Lernens zyklisch aufeinander folgen. Eine entsprechende Lesart lässt sich auf Basis der Erläuterung ebenfalls nicht belegen.

\subsubsection{Sprachfiguren und metaphorische Muster der Ad-hoc-Metaphern}

Die Analyse der Sprachfiguren und metaphorischen Muster bietet eine erste Antwort auf die Frage, mit welchen Metaphern Studierende ihre Vorstellungen vom Lehren und Lernen beschreiben. Der Begriff Sprachfigur wird für kohärente Bedeutungsübertragungen von einem Bildbereich zu einem Zielbereich verwendet. Metaphorische Muster werden als Bündelung ähnlicher Sprachfiguren verstanden (Kap. 7.3.3). Als Datenbasis dienten 22 Ad-hoc-Metaphern welche von Studierenden zur Aufgabenstellung «Lehren und Lernen im Unterricht ist wie ...» formuliert und erläutert wurden (Kap. 6.6.1.1). Mithilfe des skizzierten Vorgehens zur Metaphernanalyse (Kap. 7.3.3) konnten vier metaphorischer Muster unterschieden werden.

- Lernen und Lehren ist eine SICHTBARE FORTBEWEGUNG NACH PLAN (Kap.7.4.3.2.1)

- Lehren ist MATERIALBEARBEITUNG UND OBJEKTBEWEGUNG (Kap. 7.4.3.2.2)

- Lehren und Lernen ist GARTENARBEIT UND WACHSTUM (Kap. 7.4.3.2.3)

- Lehren und Lernen ist GEBEN UND NEHMEN (Kap. 7.4.3.2.4)

Zur kompakten Darstellung der metaphorischen Muster wird in Anlehnung an Gropengießer (2004) der Zielbereich über das Wort «ist» mit dem Bild- bzw. Quellbereich verknüpft. Die Formulierung «ist» wird dabei abkürzend für «wird metaphorisch beschrieben als» verwendet. Die unterschiedlichen Abstraktionsgerade der einzelnen metaphorischen Muster ergeben sich durch das vielfältige Datenmaterial und dessen unterschiedliche Ausdrucksformen und Präzisierungen (Kap. 7.4.3.1). Neben den identifizierten sprachlichen Mustern werden Hinweise auf weitere vereinzelt artikulierte Sprachfiguren skizziert und die Relevanz der Ergebnisse markiert (Kap. 7.4.3.2.5). 


\subsection{Lernen und Lehren ist eine SICHTBARE FORTBEWEGUNG NACH PLAN}

Das metaphorische Muster «Lernen und Lehren ist eine SICHTBARE FORTBEWEGUNG NACH PLAN» fokussiert drei Aspekte: (1.) die Fortbewegung der Lehrenden und Lernenden steht als Metapher für den Lehr- und/oder Lernprozess, (2.) diese Fortbewegung erfolgt nach einem Plan als Metapher für Lehr- und Lernziele und (3.) in unterschiedlicher Weise wird die Metaphorik des Sehens gebraucht, sowohl als Aspekt des Lehrens als auch als Reflexion des Lernens. Diese drei Schwerpunkte werden in vier Ad-hoc-Metaphern (Lehren und Lernen ist wie Wandern (D15-14), «... einen Berg zu erklimmen» (D15-16), «... eine Busfahrt» (D15-02) 226, «... ein Reiseführer» (D15-19) 227) in unterschiedlicher Weise akzentuiert.

Lernen wird in den verschiedenen Metaphern jeweils als eine Form der Fortbewegung beschrieben. Dies zeigt sich in Form expliziter Übertragungen wie beispielsweise «Reise: Lernprozess» (D15-19) oder "geht «bergauf -> man lernt immer dazu (Höhe $=$ Wissen)» (D15-16). Die Aktivität der Lernenden wird in den Ad-hoc-Metaphern unterschiedlich akzentuiert. So wird die aktive Rolle der Lernenden durch den Begriff der «Anstrengung» (D15-16) hinsichtlich des zu erklimmenden Berges erkennbar. Eine ebenfalls aktive Rolle übernehmen die Lernenden bei den Metaphern des Wanderns (D15-14) sowie des Reiseführers (D15-19). Dies zeigt sich zum einen an den zu bewältigenden Problemen beim Beschreiten des Weges, wie z. B. der holprige Weg (D15-19) oder den «eingeschränkten Bedingungen (z. B. zu steil)» (D15-14). Zum anderen zeigt sich dies auch an der Freude über zurückgelegte Meilen (D15-14). Im Bild der Busfahrt (D15-02) lässt sich die Formulierung «der Lernende sitzt in dem Bus» hingegen als Indiz dafür werten, dass den Lernenden eine eher passive Rolle zugeschrieben wird. So impliziert das Sprachbild, dass die Lernenden vom Bus gefahren werden ohne sich eigenständig bewegen zu müssen.

In den Ad-hoc-Metaphern wird Lernen in Beziehung zum Lehren über die Ziele der Fortbewegung und die geplante Erreichung dieser Ziele dargestellt. Dies erfolgt in unterschiedlicher Akzentuierung über explizite und implizite Metaphern. In der Metaphorik der Busfahrt erscheint vor allem der Lehrende in der Verantwortung für die Ziele zu stehen. Er ist es, der die Lernenden zu den «richtigen Haltestellen» fährt und er «legt Zwischenziele fest und zeigt das Endziel an». So kann das Sprachbild der «richtigen Haltestellen» dahingehend gedeutet werden, dass das Lehren und Lehren im Unterricht durch einen (Busfahrt-)Plan strukturiert wird. Die Bezeichnungen unterschiedlicher Zielformen lassen sich sowohl in der Alltagssprache als auch in der Berufs- und Wissenschaftssprache von Pädagoginnen und Pädagogen für Lehr- und Lernziele markieren, wobei nur der Lehrende für das Festlegen der Zielstellungen

226 Der Vergleich wurde zur Übersichtlichkeit gekürzt. Das Original lautet: Lehren und Lernen im Unterricht ist wie «eine Busfahrt mit den richtigen Haltestellen und einem definierten Ziel» (D15-02).

227 Der Vergleich wurde zur Übersichtlichkeit gekürzt. Das Original lautet: Lehren und Lernen im Unterricht ist wie «wenn ein Reiseführer seine buntgemischte Reisegruppe auf holprigen Wegen durch den Nebel führt» (D15-19). 
benannt wird. Das Vorhandensein von Zielen sowie eines Plans findet sich auch den weiteren Ad-hoc-Metaphern. In der Metaphorik des zu erklimmenden Berges, ist der Aufstieg des Berges bzw. das Erreichen der Spitze des Berges bereits implizit als Ziel gesetzt, ohne das beispielsweise expliziert wird, wer sich für den Berg entschieden hat. Ferner wird der Hinweis formuliert: Eine «Wanderung muss gut geplant sein» (D15-16). Implizite Metaphern die ebenfalls auf den Ursprungsbereich der geplanten Fortbewegung hindeuten, wurden über den Lehrplan zum Ausdruck gebracht. In der Formulierung «Reiseführer: Lehrer (Lehrt nach dem Plan)» (D15-19) wird Lehren vor allem als geplante Handlung dargestellt. Im Sprachbild der Wanderung deutet die Formulierung «zurückgelegte Meilen (Lehrplan)» darauf hin, dass der zurückgelegte Weg nicht beliebig war, sondern durch den «Lehrplan» strukturiert war. Inwiefern das Lehren nach Plan sowie der zurückgelegte Weg für eine Unterrichtsplanung des Lehrers oder dem Rahmenlehrplan für ein Schulfach einer Jahrgangsstufe entspricht, bleibt in den Formulierungen offen.

Die Verwendung der Metaphorik des Sehens zur Beschreibung des Lehr- und Lernprozesses ist eine weitere Gemeinsamkeit des Musters. Dabei erscheint der Rückblick auf den zurückgelegten Weg sowie der durch die Wanderung möglich gewordene Ausblick als reflexive Auseinandersetzung mit dem Ziel. Dieser Bezug erfolgt sowohl in positiv als auch negativ konnotierten Narrationen. Während die Szene «oben angekommen hat man guten Ausblick» (D15-16) als Erweiterung der Perspektive verstanden werden kann, birgt schlechtes Wetter die Gefahr, dass man «die zurückgelegte Strecke nicht erkennen und anerkennen» (D15-14) kann. Der Rückblick auf den zurückgelegten Weg als Reflexion des Lehr-Lernprozess ist in der Beschreibung aufgrund äusserer Bedingungen nicht möglich und wird als Problem markiert. Zudem wird die Metaphorik des Sehens mit der Aufgabe der Lehrenden verbunden. Die Formulierung, der Lehrende «zeigt das Endziel an», bringt in der Busfahrt-Metapher zum Ausdruck, dass der Lehrende nicht nur die Ziele festlegt, sondern sie auch für die Schülerinnen und Schüler sichtbar macht, indem er sie anzeigt. In der Metaphorik des Reiseführers (D15-14) wird der explizite Vergleich formuliert «Nebel: Lernstoff (Am Anfang noch unverstanden)». Die Aufgabe der Lehrenden wird dahingehend beschrieben, dass dieser die Lernenden «durch den Nebel führt», so dass «alle wieder klar sehen können». Der durch die Reise neu gewonnene Durchblick kann ebenfalls als Erweiterung der Perspektive im Sinne des durch den Bergaufstieg möglichen Ausblicks verstanden werden.

\subsection{Lehren ist MATERIALBEARBEITUNG UND OBJEKTBEWEGUNG}

Das Muster «Lehren ist MATERIALBEARBEITUNG UND OBJEKTBEWEGUNG» fokussiert zwei Aspekte: (1.) Lernende werden als passive (und nicht lebendige) Objekte und Materialien konzeptualisiert, welche (2.) von Lehrenden bearbeitet, verändert bzw. 
verbessert werden. Diese zwei Schwerpunkte werden in drei Ad-hoc-Metaphern (Lehren und Lernen ist wie ein Bäcker mit seinen Zutaten und Rezepten (D15-18), «... ein Papierflugzeug / ein Drachen» (D15-17) $)^{228}$, «... Schneeballkugel» (D15-21) ${ }^{229}$ ) mit unterschiedlichen Bildbereichen und Akzentuierungen veranschaulicht.

Lehrende sind in diesem metaphorischen Muster die aktiv Handelnden, welche ihr Vorgehen in unterschiedlicher Weise planen und strukturieren können sowie sich für verschiedene Handlungsalternativen entscheiden können bzw. müssen. Dies wird in den Vergleichen unterschiedlich zum Ausdruck gebracht. Dem Bäcker werden beispielsweise unterschiedliche Handlungsstrategien zugeschrieben: «der Lehrende (Bäcker) hat mündlich viele Rezepte/ Wege den Schüler zu belehren» (D15-18). Der Rezeptvergleich legt die Lesart nahe, dass das Lehren in einer durch das Rezept strukturierten bzw. geplanten Weise erfolgt. In der Konstruktion des Papierflugzeuges werden im Bildbereich ebenfalls Aspekte der Planung sowie unterschiedliche Handlungsmöglichkeiten skizziert. So «kann viel oder wenig Planung im Bau stecken» und die Formulierung «Die Art des Werfenden» lässt den Schluss zu, dass unterschiedliche Möglichkeiten des Werfens existieren ${ }^{230}$. Im Vergleich des Lehrens als zu rollende Schneekugel, sind ebenfalls die Lehrenden die aktiv Handelnden: «Kugelanschieber» bzw. «Roller = Lehrer». Im Gegensatz zu den zwei vorherigen Vergleichen werden keine Aspekte der Planung expliziert. Auch die unterschiedlichen Möglichkeiten zu handeln bzw. eine Kugel zu rollen, scheinen nicht frei wählbar, sondern sind abhängig von äusseren Rahmenbedingungen: «Muss manchmal bergauf rollen (Kraft) oder Umwege nehmen oder auch rollen lassen (bergab)» (D15-21). Die Vorstellung, dass Lehren als Veränderung und Bewegung von passiven Lernenden möglich ist, findet sich in den drei benannten Ad-hoc-Metaphern. Zweifel an bzw. ein Hinterfragen der Wirkmächtigkeit des Lehrenden werden kaum thematisiert. Grenzen des Lehrenden werden implizit im Umfeld und den Bedingungen sowie den Voraussetzungen der Lernenden gesehen.

Lernende werden in diesem Muster zum einen als Rohmaterial für einen Fertigungs- bzw. Bearbeitungsprozess verstanden: als Zutaten für einen Bäcker (D15-18) sowie als Material des Papierflugzeugs (D15-17). Zum anderen werden Lernende als bewegliche Objekte konzeptualisiert, welche Lehrende bewegen können, als ein $\mathrm{Pa}$ pierflugzeug (D15-17) sowie als «Kugel = Lernender» (D15-21). Gemeinsam ist diesen Formulierungen, dass Lernende als weitgehend passive Objekte eines Lehrprozesses verstanden werden. Die Fokussierung auf den Lehrprozess zeigt sich u. a. dadurch, dass entweder Lernen nicht thematisiert wird oder als Folge eines vorherigen

228 Allerdings wurde in der Erläuterung nicht auf die Drachenmetaphorik eingegangen.

229 Der Vergleich wurde zur Übersichtlichkeit gekürzt. Das Original lautet: Lehren und Lernen im Unterricht ist wie «[eine] Schneeballkugel, die über hügelige Wiese gerollt wird, auf der Schnee liegt» (D15-21).

230 In Rahmen des Vergleichs bleibt allerdings offen, inwiefern die jeweiligen Lehrenden über die Art des Werfens selbst entscheiden können bzw. inwiefern die Art des Werfens für einen bestimmten Lehrenden steht. 
Lehrprozesses verstanden wird. Die Passivität der Lernenden zeigt sich implizit im Vergleich mit nicht lebendigen Objekten. Ferner wird die Passivität der Lernenden dadurch zum Ausdruck gebracht, dass die Objekte bewegt bzw. verändert werden und sich nicht eigenständig bewegen oder verändern können. Die Schneeballkugel beispielsweise «rollt manchmal alleine» (D15-21) aber benötigt den «Roller = Lehrer» (D15-21). Diese Passivität zeigt sich auch im Vergleich mit einem Papierflugzeug. Die Formulierung «Die Art des Werfenden und [das Flugzeug] selbst beeinflussen den Flug» eröffnet zwar die Lesart, dass die Lernenden selbst einen Einfluss auf den Prozess des Fliegens haben. Allerdings impliziert das Bild des geworfenen Papierflugzeuges auch, dass ein Papierflugzeug ohne den Wurf des Lehrenden gar nicht fliegt.

\subsection{Lehren und Lernen ist GARTENARBEIT UND WACHSTUM}

Das metaphorische Muster «Lehren und Lernen ist GARTENARBEIT UND WACHSTUM» fokussiert zwei Aspekte: (1.) Lehren wird als Gartenarbeit konzeptualisiert mit dem Ziel (2.) Lernenden als Pflanzen angemessene Bedingungen zum Wachsen zu schaffen. Diese zwei Schwerpunkte werden in zwei Ad-hoc-Metaphern (Lehren und Lernen ist wie Gartenarbeit (D15-22), und «... Gärtnern» (D15-01) ${ }^{231}$ ) mit unterschiedlichen Akzentuierungen und unterschiedlicher Stringenz veranschaulicht.

Die Lehrenden bzw. das Lehren stehen im Fokus dieses Musters. Lehrende werden jeweils mit Gärtnern (D15-01, D15-22) verglichen. Als Tätigkeiten des Gärtners werden - im Bildbereich bleibend - Tätigkeiten des Pflanzens, Pflegens und Gießens (D15-22) genannt. Zugleich wird zur Erläuterung dieser Tätigkeiten von den Studierenden auf weitere Bildbereiche zurückgegriffen. In der Formulierung «Gießen als Input» (D15-22) wird beispielsweise der Begriff des Inputs scheinbar selbsterklärend verwendet. Allerdings kann Input auch als implizite Metapher verstanden werden, mit der Lehren als Eingabe oder Mitgeben von Wissen, Fähigkeiten oder Ähnlichem verstanden wird. Bei der Formulierung «Der Gärtner gibt seinen Pflanzen alles mit auf den Weg um sich gut entwickeln und wachsen zu können» (D15-01) ${ }^{232}$ werden zur Beschreibung der Gärtnertätigkeiten implizit auch die Bildbereiche der Fortbewegung sowie des Gebens und Nehmens verwendet ${ }^{233}$. Gemeinsam sind diesen Formulierungen, dass Lehrenden eine aktive und handelnde Rolle zugeschrieben werden. Im Gegensatz zu dem vorherigen metaphorischen Muster «Lehren als MATERIALBEARBEITUNG UND OBJEKTBEWEGUNG» stehen Lehrende jedoch in einer anderen

231 Der Vergleich wurde zur Übersichtlichkeit gekürzt. Das Original lautet: Lehren und Lernen im Unterricht ist wie «Gärtnern/ein Gärtner bei der Gartenarbeit/Gartenarbeit» (D15-02).

232 Wenngleich die Formulierung so gelesen werden kann, dass es um die Entwicklung der Gärtners geht, wurde sich im Rahmen der kommunikativen Validierung dazu entschieden, die Entwicklung auf die Pflanzen zu beziehen.

233 Die Frage, warum dies als einheitlich gewertet wird, begründet sich vor dem Hintergrund, dass weiteren Bildbereiche jeweils nur einmal verwendet werden, während die Gartenarbeit und WachstumsMetaphorik häufiger verwendet wird. 
Relation zu ihren Lernenden. Die Tätigkeiten des Lehrenden zielen nicht direkt auf die Veränderung der Pflanzen bzw. Lernenden, sondern auf die Gestaltung der Rahmenbedingungen ab. Damit wird der Zusammenhang zwischen Lehren und Lernen nicht als kausale Abhängigkeit verstanden: Der «Gärtner kann gewisse Rahmenbedingungen festlegen, kann letztendlich nicht über Qualität des Endproduktes entscheiden» (D15-22). Dabei unterscheiden sich die zwei Metaphern hinsichtlich der Begründung der Gartenarbeit bzw. hinsichtlich des Ergebnisses. Die Formulierung «Qualität des Endproduktes» (D15-22) lässt Gartenarbeit einerseits als eine Art Fertigungsprozess erscheinen. Andererseits dient die Gartenarbeit dem Zweck, Pflanzen Möglichkeiten zu schaffen «um zu wachsen, aufzublühen, sich gut zu entwickeln» (D15-01), somit scheint vielmehr die Entwicklung der Pflanzen selbst das Ziel zu sein.

Einen «Schüler als Pflanze» (D15-22) zu betrachten, ist die zentrale Gemeinsamkeit dieses Musters. Entsprechend sind Lernende lebendig und keine leblosen Objekte, wie im metaphorischen Muster Lehren als MATERIALBEARBEITUNG UND OBJEKTBEWEGUNG. Lernenden wird in diesem Bild stattdessen das Potenzial zugeschrieben, eigenständig wachsen und sich entwickeln zu können. Das Lernen der Schülerinnen und Schüler kann als Wachsen der Pflanzen verstanden werden. Zugleich wird Lernen im Rahmen dieses Musters nicht näher präzisiert und die Handlungsmöglichkeiten der Lernenden bleiben weitgehend unbestimmt. Im Vergleich mit dem metaphorischen Muster «Lernen und Lehren ist eine SICHTBARE FORTBEWEGUNG NACH PLAN» haben Lernende im Bildbereich der Gartenarbeit beispielsweise keine Artikulations- oder Interaktionsmöglichkeiten mit dem Lehrenden. Während Lernenden als lebendige und eigenständig wachsende Lebenswesen einerseits eine aktive Rolle als Lernenden unterstellt werden kann, sind ihre Möglichkeiten sich partizipativ am Unterricht beteiligen zu können, aufgrund fehlender Interaktionsmöglichkeiten, deutlich beschränkt.

\subsection{Lehren und Lernen ist GEBEN UND NEHMEN}

Das metaphorische Muster «Lehren und Lernen ist GEBEN UND NEHMEN» hebt insbesondere hervor, dass Lehren und Lernen als wechselseitige Interaktionsprozesse verstanden werden. Zudem werden sowohl die Lehrenden als auch die Lernenden als Geberinnen und Geber bzw. Nehmerinnen und Nehmer verstanden. Diese zwei Schwerpunkte werden in zwei Ad-hoc-Metaphern (Lehren und Lernen ist wie "eine gute Beziehung in der sich geben und nehmen die Waage halten» (D15-12), «... was einzukaufen und zu essen» (D15-20 $)^{234}$ ) mit unterschiedlichen Bildbereichen und unterschiedlichen Schwerpunktsetzungen veranschaulicht ${ }^{235}$.

234 Der Vergleich wurde zur Übersichtlichkeit gekürzt. Das Original lautet: Lehren und Lernen im Unterricht ist wie «zum Bäcker gehen, was einzukaufen und zu essen» (D15-20).

235 Ferner findet sich die Metaphorik des Gebens und Nehmens bei weiteren Ad-hoc-Metaphern in Form impliziter Metaphern für Lehrende (z. B. «Der Gärtner gibt seinen Pflanzen alles mit» (D15-06) sowie für 
Lehren wird in diesem Muster vor allem als Prozess des Gebens verstanden und wird sowohl in Form von expliziten als auch impliziten Metaphern sprachlich zum Ausdruck gebracht. Explizit wird diese Metapher im Bildbereich des Verkaufens über das Angebot eines Verkäufers versprachlicht. Der «Verkäufer ist der Lehrende» und dieser gibt ein «Angebot/Thema» (D15-20) vor. Dabei wird das Angebot verstanden als «Vor-(Zu)bereitete Themen und Inhalte (Zutaten)» (D15-20).

Zudem wird dem Lehrenden die Aufgabe zugeschrieben, dass er das Angebot schmackhaft macht. Das Geben des Lehrenden erfolgt in dieser Ad-hoc-Metapher zum einen als das Geben einer Auswahl. Zum anderen wird das Geben als Verkaufen metaphorisiert. Dabei impliziert das Sprachbild, das der Lehrende für die jeweiligen verkauften Backwaren auch etwas nimmt. Dies bleibt im Rahmen der Ad-hoc-Metapher unbestimmt. Im Bildbereich der «Beziehung, in der sich geben und nehmen die Waage halten» (D15-12) wird der Prozess des Gebens direkter formuliert: «Der Lehrer teilt sein Wissen, gibt seine Erfahrung an die Schüler weiter» (D15-12). Inwiefern die Schülerinnen und Schüler die Entscheidungsmöglichkeiten haben diese weitergegebene Erfahrung anzunehmen, wird im Rahmen der Ad-hoc-Metapher nicht expliziert. In diesem Sprachbild ist die Tätigkeit des Lehrenden nicht auf die Weitergabe beschränkt. Stattdessen «nimmt [der Lehrende] aber auch ebenso Impulse von den Schülern auf» (D15-12).

Lernen wird in den zwei Bildbereichen unterschiedlich präzise bestimmt. Im Rahmen der Ad-hoc-Metapher der Beziehung werden keine expliziten Aussagen über die Lernenden formuliert. Die Erläuterungen der Ad-hoc-Metaphern implizieren bzw. legen die Vermutung nahe, dass Lernende die weitergegebenen Erfahrungen des Lehrenden aufnehmen und ihrerseits dem Lehrenden Impulse geben. Im Rahmen der Ad-hoc-Metapher «... was einzukaufen und zu essen» wird der Lernende explizit als Käufer metaphorisiert. Dieser «wählt aus, entscheidet selbst, was von dem Angebot er an- und schließlich auch aufnimmt» (D15-20).

\subsection{Weitere Sprachfiguren und Sprachbilder}

Neben den skizzierten metaphorischen Mustern werden in den Ad-hoc-Metaphern der Studierenden weitere Sprachfiguren zum Lehren und Lernen im Unterricht zum Ausdruck gebracht. Diese einzelnen Sprachfiguren eröffnen ein vielfältiges Spektrum an unterschiedlichen Bildbereichen. Lehren und Lernen im Unterricht wird $u$. a. verglichen mit konkreten Objekten, wie z. B. einem «Uhrwerk» (D15-04) oder einem «Fenster» (D15-06). Ferner werden das Lehren und Lernen im Unterricht explizit mit spezifischen Tätigkeiten verglichen. Beispiele sind u. a. der Blick über den Tellerrand

Lernende (z. B. «Wenn die Lernenden das Fenster offen lassen, bekommen sie vieles für die Zukunft mit» (D15-06) oder «wenn die gesendeten Lehrinhalte entgegengenommen und verarbeitet werden» (D15-13). 
(D15-10), der «Gang auf dünnem Eis» (D15-09) oder das «Häuser bauen ohne Bauplan» (D15-08) sowie das «Senden und Empfangen» (D15-13). Mit den verschiedenen Bildbereichen geht auch die Metaphorisierung sehr unterschiedlicher Aspekte zu Lehren und Lernen einher. Mit der Metapher vom «Gang auf dünnem Eis» (D1509) wird u. a. das "Klima in der Klasse» sowie das Verhältnis zwischen Lehrkräften und Lernenden thematisiert. Bei den Ad-hoc-Metaphern «Blick über den Tellerrand» (D15-10) sowie "Sendens und Empfangen» (D15-13) werden insbesondere die Vorstellungen über das Lernen und Lehren thematisiert. Durch diese unterschiedlichen Bildbereiche werden auch unterschiedliche Lernvorstellungen sichtbar. In der Metapher «Senden und Empfangen» (D15-13) werden Lehrinhalte beim Lernen nahezu mechanisch «entgegengenommen und verarbeitet» (D15-13). In der Metapher des «Blick[s] über den Tellerrand» (D15-10) kann Lernen hingegen als «Erweiterung des Horizonts» sowie als «Entdeckung neuer Perspektiven» (D15-10) beschrieben werden.

\subsubsection{Darstellungsformen und Strukturelemente der Gruppenmetaphern}

Ein zentraler Anlass zur Diskussion und Auseinandersetzung mit alternativen Sprachund Denkfiguren zum Lehren und Lernen bestand in der Aufgabenstellung, als studentische Arbeitsgruppe eine Metapher zum Lehren und Lernen zu entwickeln. Die konkrete Aufgabenstellung lautete: «Schreiben Sie als Gruppe Ihren Beitrag für das Wikibook. Ziel des Beitrages ist es, Ihre Vorstellung vom Lehren und Lernen als Metapher auszudrücken sowie die Grenzen und Probleme der Metapher deutlich zu machen» (Kap. 6.6.1.1). Zur einheitlichen Gestaltung der Wikibookseiten wurden gemeinsam mit den Studierenden verschiedene Gestaltungsprinzipien vereinbart (Kap. 7.2.2). Im Folgenden wird der Umgang der Studierenden mit diesen Gestaltungsprinzipien anhand der erstellten Wikibeiträge exemplarisch erläutert:

- Formulierung eines prägnanten expliziten Vergleichs (Kap. 7.4.3.3.1)

- Tabellarische Darstellung expliziter Bedeutungsübertragungen (Kap. 7.4.3.3.2)

- Erläuterung der Gruppenmetapher in Form des Fliesstextes (Kap. 7.4.3.3.3)

- Thematisierung von Grenzen der Metaphorik (Kap. 7.4.3.3.4)

Die benannten Darstellungsformen bringen metaphorische Bedeutungsübertragungen in unterschiedlicher Weise zum Ausdruck und eröffnen so unterschiedliche Artikulations- und Reflexionspotenziale. Am Beispiel der studentischen Schreib- und Textprodukte wird dabei auf die jeweiligen Besonderheiten des Materials aufmerksam gemacht. 


\subsection{Formulierung eines prägnanten expliziten Vergleichs}

In Anlehnung an die Aufgabenstellung der individuell zu formulierenden Ad-hoc-Metaphern zu Beginn des Seminars (Kap. 6.6.1.1) wurden die Studierenden in Gruppen dazu aufgefordert, ihre Metaphern in Form eines prägnanten expliziten Vergleichs zu formulieren. Für die Gestaltung des Wikibooks sollte dieser Vergleich vor allem zur Referenzierung der jeweiligen Gruppenmetaphern dienen.

Bei Betrachtung des Wikibooks und der studentischen Beiträge zeigt sich, dass diese Aufgabe in unterschiedlicher Weise bewältigt wurde. Der vom Zeichenumfang kürzeste explizite Vergleich lautete «Lehren und Lernen ist wie Wandern». Der Vergleich wurde in der Form sowohl als Seitentitel sowie zur Referenzierung im Inhaltsverzeichnis verwendet. Nach diesem Vorgehen erfolgte auch die Bezeichnung von sechs weiteren Gruppenmetaphern ${ }^{236}$ (Abb. 7.12).

\section{Vorstellungen und Metaphern [Bearbeiten ]}

2.1 Lehren und Lernen ist wie... [ Bearbeiten ]
2.1.1 ...von einem Reisebegleiter begleitet zu werden
2.1.2 ...Wandern
2.1.3 ...von einem Reiseführer begleitet werden
2.1.4 ...Häuser bauen ohne konkreten Bauplan
2.1.5 ...der Garten des Lebens
2.1.6 ...Gärtnern und Gedeihen
2.1.7 ...ein Besuch in einer Bäckerei
2.1.8 ...Senden und Empfangen

Abb. 7.12: Screenshot zum Inhaltsverzeichnis zum zweiten Kapitel mit den expliziten Vergleichen (online abgerufen am 31. September 2015).

\subsection{Tabellarische Darstellung expliziter Bedeutungsübertragungen}

Ein weiteres Strukturierungsmerkmal zur Gestaltung die Wikiseiten bestand darin, die zentralen Bedeutungsübertragungen vom jeweils gewählten Bildbereich zum Zielbereich des Lehrens und Lernens tabellarisch zum Ausdruck zu bringen (z. B. Abb. 7.13). Dieses Format wurde den Studierenden bereits im Rahmen des Seminars zur

236 Die Ausnahme bildet der vom Zeichenumfang längste explizite Vergleich. Dieser lautete: «Lehren und Lernen ist wie, wenn ein Reiseführer seine buntgemischte Reisegruppe auf holprigen Wegen durch den Nebel führt, bis alle wieder klar sehen können und die Wege wieder eben sind». Für die Bezeichnung der Wikibookseite wählte die Gruppe den Titel «Reiseführermetapher» und für die Referenzierung im Inhaltsverzeichnis die Formulierung «Lehren und Lernen ist wie von einem Reiseführer begleitet [zu] werden». 
Flipchartgestaltung für die Darstellung und Diskussion der erarbeiteten Gruppenmetaphern empfohlen und von ihnen realisiert ${ }^{237}$.

\begin{tabular}{|l|l|}
\hline \multicolumn{1}{|c|}{ Metapher } & \multicolumn{1}{c|}{ Bedeutung } \\
\hline Baumanagement & die Lehrkraft \\
\hline Handwerker & heterogene Gruppe von Lernenden \\
\hline Bauplan & Lehrplan \\
\hline Bauziel/ fertiges Bauprojekt & Lernziel \\
\hline Bauamt & Kultusministerium/ Schulamt \\
\hline Material & individuelles Feinziel \\
\hline Bauprozess & Lernprozess \\
\hline überwachen & zum Lernziel hinführen \\
\hline
\end{tabular}

Abb. 7.13: Screenshot der tabellarischen Übersicht der übertragenden Elemente vom Bildbereich (Metapher) zum Zielbereich (Bedeutung) (G15-04).

Die veröffentlichten Wikiseiten der Gruppenmetaphern umfassen tabellarische Übersichten mit jeweils sechs bis acht explizit formulierten Elementen, die als Bedeutungsübertragung zwischen Bild- und Zielbereich verstanden werden können. Interessant dabei ist, wie selbstverständlich routinisierte metaphorische Redewendungen von den Studierenden als Fachsprache des Lehrens und Lernens verwendet werden. Im Beispiel (Abb. 7.13) zeigt sich dieses Phänomen an der Bedeutungsübertragung von «überwachen = zum Lernziel hinführen» (G15-04). Da die Rhetorik der Führung in der pädagogischen Literatur verwendet wird (Kap. 5.4.1, 5.5.1), mag diese Formulierung nicht überraschend sein. Gleichwohl bietet sich ein Diskussionsanlass zur Frage, warum Studierende in diesem Zusammenhang eine Fortbewegungs-/Führungsmetaphorik verwenden, da diese als Bildbruch zum Bauen eines Hauses interpretiert werden kann. Das Phänomen der Verwendung routinisierter Metaphern zur Erklärung expliziter Bedeutungsübertragungen findet sich auch in weiteren Gruppenmetaphern (Kap. 7.4.3.4).

\subsection{Erläuterung der Gruppenmetapher in Form des Fliesstextes}

Die Erläuterung der Metapher erfolgte durch die Studierenden jeweils als Fliesstextes. Die Beiträge der Studierenden umfassen zwischen 2094 und 6094 Zeichen (inklusive Leerzeichen). Die Darstellungsformen zur Erläuterung können als szenische Beschreibungen, explizite Bedeutungsübertragungen und Mischformen beschrieben

237 Ferner kann das Darstellungsformat als formalisierte Variante der Darstellungsform «Explizite Benennung der übertragenden Elemente» (Kap. 7.4.3.1) der Ad-hoc-Metaphern verstanden werden. 
werden (Kap. 7.4.3.1). Zwischen diesen Formen wird in der Darstellung häufig gewechselt, sodass beispielsweise eine szenische Beschreibung im Bildbereich der Metapher erfolgt, welche im folgenden (Halb-)Satz explizit auf den Zielbereich des Lehrens und Lernens übertragen wird.

«Wenn ein Kunde in eine Bäckerei geht, schaut er sich das Angebot an, wobei der Verkäufer beratend zur Seite steht und nähere Auskunft über das Angebot geben kann. Der Kunde, der in die Bäckerei geht, steht hierbei für einen Lernenden, der in eine beliebige Lehr-/Lerninstitution geht» (G15-07).

Während der erste Satz als szenische Beschreibung im Bildbereich zunächst keinen Bezug zum Lehren und Lernen aufweist, wird dieser im folgenden Satz erläutert. Neben der expliziten Bedeutungsübertragung, «Der Kunde [...] steht hierbei für einen Lernenden» (G15-07), lässt der folgende Halbsatz die Lesart zu, dass das Hineingehen in die «Bäckerei» (G15-07) auch dem Hineingehen in eine «beliebige Lehr-/ Lerninstitution» (G15-07) entspricht. Zudem finden sich in den Erläuterungen verschiedene Mischformen (Kap. 7.4.3.1). Diese Mischformen lassen sich zum einen auf formaler Ebene identifizieren, z. B. bei Metaphern bei denen szenische Beschreibungen und explizite Vergleiche ineinander übergehen. Zum anderen findet sich auch in den Erläuterungen der Metaphern das Phänomen, dass routinisierte Metaphern von Studierenden zur Erläuterung des Zielbereichs verwendet werden.

\subsection{Thematisierung von Grenzen der Metaphorik}

Im Rahmen der Erläuterung wurden die Studierenden aufgefordert, die Grenzen und Probleme der Metaphorik zu thematisieren. Dafür wurden im Rahmen des Seminars mögliche Widersprüche in Form erkennbarer Bildbrüche sowie Aspekte des Highlighting und des Hiding (Kap. 5.3.3) diskutiert. So stand in den Gruppenpräsentationen jeweils in Frage, welche Facetten des Lehrens und Lernens durch die jeweilige Metapher angemessen und welche gar zum Ausdruck gebracht werden können. Im Rahmen ihrer Wikitexte thematisierten die studentischen Arbeitsgruppen die Grenzen und Probleme sehr unterschiedlich. Während einige Gruppen keine entsprechende Betrachtung ausformulierten (G15-01 ${ }^{238}, \mathrm{G} 15-05, \mathrm{G} 15-06, \mathrm{G} 15-08^{239}$ ), erfolgte bei anderen Gruppen die Benennung einzelner ausgewählter Problematiken bzw. eine Diskussion über verschiedene Grenzen und offene Fragen zur entwickelten Metapher (G15-02, G15-03, G15-04, G15-07) ${ }^{240}$.

238 Eine Betrachtung der Grenzen erfolgte von dieser Gruppe hinsichtlich der Handlungsmöglichkeiten im Bildbereich, aber nicht bezogen auf die Metaphorik.

239 Dies ist insofern interessant, weil in der Diskussion sowie auf dem selbst vorbereiteten Flipchart der Gruppe verschiedene Probleme thematisiert wurden.

240 Eine exemplarische Diskussion wurde wie folgt geführt: «So wie andere Metaphern, ist auch diese nur begrenzt für das Lehren und Lernen im Unterricht anzuwenden. So ist es zwar in manchen Fällen gut, 


\subsubsection{Sprachfiguren und metaphorische Muster der Gruppenmetaphern}

Im Rahmen des Seminars wurden acht Gruppenmetaphern zum Lehren und Lernen von studentischen Kleingruppen zwischen zwei und fünf Personen formuliert. Zur Erstellung einer strukturieren Übersicht der Sprachfiguren sowie der metaphorischen Muster wurden die Gruppenmetaphern analysiert (Kap. 7.3.3). Analog zur Darstellung der Ad-hoc-Metaphern werden Gruppenmetaphern, welche Gemeinsamkeiten hinsichtlich der Übertragung von einem Bildbereich auf einen Zielbereich aufweisen, im Folgenden als metaphorische Muster zusammengefasst. Einzelne Gruppenmetaphern, die eine eigene bzw. von den anderen Metaphern abgrenzbare Metaphorik entfalten, werden im Folgenden als Sprachfiguren bezeichnet. Es wird zwischen fünf metaphorischen Mustern und Sprachfiguren in den Gruppenmetaphern unterschieden:

- Lernen und Lehren ist eine SICHTBARE FORTBEWEGUNG MIT HINDERNISSEN ( $n=3)$.

- Lehren und Lernen ist GARTENARBEIT UND WACHSTUM $(n=2)$.

- Lehren und Lernen ist EIN BESUCH IN EINER BÄCKEREI $(n=1)$.

- Lehren und Lernen ist HÄUSER BAUEN OHNE KONKRETEN BAUPLAN $(n=1)$.

- Lehren und Lernen ist SENDEN UND EMPFANGEN $(n=1)$.

Das Muster «Lernen und Lehren ist eine SICHTBARE FORTBEWEGUNG MIT HINDERNISSEN» basiert auf drei Gruppenmetaphern (G15-01, G15-02, G15-03). Die tabellarische Übersicht der jeweiligen Gruppenmetaphern ist im Anhang dargestellt (Abb. B.5). Das Muster stellt eine veränderte Akzentuierung des metaphorischen Musters "SICHTBARE FORTBEWEGUNG NACH PLAN» der Ad-hoc-Metaphern dar (Kap. 7.4.3.2). Auf Basis der Ausdifferenzierungen der Gruppenmetaphern lassen sich für das Muster die folgenden konstitutiven Merkmale skizzieren: (1.) Lernende werden als (eine Gruppe von) Menschen metaphorisiert, die entlang verschiedener Routen und Wege spezifische Ziele erreichen wollen bzw. sollen, z. B. als «Reisegruppe» (G15-01, G1503) oder Wanderer» (G15-02). Die Fortbewegung zum Ziel als «Reisen» (G15-01, G15-03) und «Wandern» (G15-02) wird als Lernen verstanden. (2.) Schwierigkeiten und Hindernisse entlang der Routen und Wege, z. B. in Form von «Schlaglöcher[n]» (G15-01, G15-03) oder «bei schlechtem Wetter» (G15-03), stehen für mögliche Lernschwierigkeiten sowie für die unterschiedlichen Voraussetzungen der Lernenden. (3.) Lehrende werden in den Gruppenmetaphern als «Reiseführer» (G15-01) bzw. «Reisebegleiter»(G15-02) sowie als «Infopoint» (G15-03), welche die Lernenden in unterschiedlicher Weise beim Erreichen der Ziele unterstützen, metaphorisiert.

wenn der Lernende vom vorgegebenen Weg abkommt und durch ein individuelles Vorgehen das Lernziel erreicht, es ist jedoch unklar, wie vorgegangen wird, wenn sich ein Schüler komplett verirrt und es ihm nicht von alleine gelingt, wieder auf den richtigen Pfad zu kommen. Da die Lehrperson in dieser Metapher nur an den festgelegten Infopoints als Helfer zur Verfügung steht, stellt sich die Frage, in wieweit sie in diesem Fall eingreifend wirkt. Eine weitere Grenze der Metapher stellt der Infopoint als Informations-und Entscheidungsmedium da[r], denn dieser tritt bei Auftreten des Lehrers in den Hintergrund und kann nicht präzise gefasst werden» (G15-02). 
Lehren umfasst in diesem Muster ein Spektrum von Metaphorisierungen. Dazu gehört zum einen die Bereitstellung von Orientierungshilfen und Materialien an spezifischen Orten entlang der Routen und Wege. Zum anderen beinhaltet das Spektrum die Metaphorik der Führung einer Reisegruppe. (4.) Die Metaphorik des Sehens wird in Gruppenmetaphern einerseits als Ausdruck möglicher Schwierigkeiten und anderseits als reflexives Moment des Lernens verwendet. Während «Aussichtspunkte» (G15-02) beispielsweise spezifische Ziele des Lernens darstellen, beschreibt die eingeschränkte Sicht durch «Nebel» (G15-03) eine Schwierigkeit des Lernens. Eine Reflexion der Grenzen der spezifischen Metaphern erfolgte durch die Gruppen mit unterschiedlichen Schwerpunkten. Dabei stellte sich jeweils die Frage, inwiefern alle Schülerinnen und Schüler ein spezifisches Ziel erreichen (können).

Lehren und Lernen als «GARTENARBEIT UND WACHSTUM» zu beschreiben, findet sich als Muster in zwei Gruppenmetaphern (G15-05, G15-06). Die tabellarische Übersicht der jeweiligen Gruppenmetaphern befindet sich im Anhang (Abb. B.6). Das bereits in den Ad-hoc-Metaphern identifizierte Muster (Kap. 7.4.3.2) kann bei Betrachtung der Ausdifferenzierungen der Gruppenmetaphern durch die folgenden Merkmale beschrieben werden. (1.) Lehrende werden als Gärtner metaphorisiert und das Lehren wird als Gartenarbeit beschrieben. Die Gartenarbeit wird im Bildbereich in unterschiedlichen Phasen beschrieben, welche das Säen der Samen, die Pflege der Pflanzen und die Ernte der Früchte umfassen. Ferner werden einzelne Aufgaben beschrieben, z. B. «muss der Gärtner die Pflanzen zurechtstutzen, Unkraut jäten, düngen, bewässern usw.» (G15-05). Diese Aufgaben werden verstanden als das «Schaffen einer Lernumgebung, das Befähigen und Unterstützen von Lernprozessen, aber auch Zurechtweisung» (G15-05). (2.) Lernende werden als Pflanzen metaphorisiert. Lernen entspricht in diesem Zusammenhang dem «Wachstum» (G15-05) bzw. dem «Wachsen» sowie dem «Gedeihen» (G15-06). Dabei wird jeweils zum Ausdruck gebracht, dass der Gärtner zwar die Voraussetzungen schaffen kann, jedoch «die Pflanze von alleine wachsen [muss]»(G15-06). Analog können Lehrende über die Gestaltung von Lernumgebungen beispielsweise die Voraussetzungen zum Lernen schaffen aber Lernende letztlich nicht lernend «machen». (3.) Das Wachstum der Pflanzen (bzw. das Lernen der Schülerinnen und Schüler) wird von äusseren Umwelteinflüssen beeinflusst. Diese äusseren «Bedingungen (Wind, Wetter, Boden ...)» (G15-06) werden u. a. auf das «soziale Umfeld» (G15-06) übertragen und es werden Folgen des Einflusses als Über- oder Unterforderung der Lernenden im Zielbereich ausgeführt (G15-05, G15-06).

Die Sprachfigur «Lehren und Lernen ist EIN BESUCH IN EINER BÄCKEREI» (G15-07) wurde in einer Gruppenmetapher zum Ausdruck gebracht (Anhang B.7). Diese Metaphorik wurde bereits in Form einer Ad-hoc-Metapher formuliert und kann als spezifische und zugleich erweiterte Form des Musters «Lehren und Lernen ist wie GEBEN UND NEHMEN» (Kap. 7.4.3.2) verstanden werden. Folgende Merkmale sind zentral für 
dieses Muster: (1.) Lehrende werden als Verkäufer von Backwaren verstanden. Backwaren entsprechen den zu lernenden Lehrinhalten. Die Aufgabe des Lehrenden als Verkäufer besteht darin, dass er «beratend zur Seite steht und nähere Auskunft über das Angebot geben kann»(G15-07). Davon zu unterscheiden ist ein Bäcker. Diese wird verglichen «mit einem Wissenschaftler, der forscht oder sein Wissen weiter gibt, indem er beispielsweise Bücher schreibt (= neue Rezepte)». (2.) Lernende werden in dieser Metaphorik als Kunden der Bäckerei verstanden. Lernen umfasst die Auswahl von Backwaren sowie den Verzehr von diesen. «Dies bedeutet übertragen, dass der Lernende selbst bestimmt, welche Lerninhalte er lernen möchte» (G15-07). Darüber hinaus benötigen Kunden im Bildbereich Geld, um Backwaren kaufen zu können: «Das Geld steht hierbei für die Anstrengung, welche der Kunde aufbringen muss, um den jeweiligen Lerninhalt zu verstehen und zu lernen»(G15-07). Grenzen der Metaphorik werden u. a. in der geringen Gestaltungsmöglichkeit des Lehrenden gesehen.

«Lehren und Lernen ist HÄUSER BAUEN OHNE KONKRETEN BAUPLAN» (G15-04) ist eine weitere Sprachfigur, welche in Form einer Gruppenmetapher zum Ausdruck gebracht wurde (Anhang B.8). Diese Metaphorik wurde bereits in Form einer Ad-hocMetapher formuliert (Kap. 7.4.3.2). Die Gruppenmetapher erweitert und konkretisiert die Ausführungen der Ad-hoc-Metapher. Im Rahmen dieser Sprachfigur wird insbesondere die Planbarkeit des Lehrens und Lernens thematisiert. Dafür wird zwischen einem groben und einem konkreten Bauplan unterschieden:

«[Es] sollen Häuser nach einem groben Bauplan gebaut werden (Villa, Hochhaus, Appartement). Dieser Bauplan (Lehrplan) dient zur Orientierung, um gewisse Ziele zu erreichen, die vor dem Baubeginn formuliert wurden. Während des Bauprozesses ist es von Bedeutung, dass das Lehren und Lernen unter Vorgabe des Lehrplans (Bauplans) stattfinde[n], jedoch ist die Umsetzung hinsichtlich eines individuellen Feinziels frei gestaltbar. Dieses individuelle Feinziel wird von jedem Schüler, der im Fall des Bauprozesses als Handwerker fungiert, in einem individuellen Prozess gestaltet» (G15-04).

Lehrerinnen- und Lehrer werden in dieser Sprachfigur als «Baumanagement» (G15-04) metaphorisiert. Zu den Aufgaben des Baumanagements gehört die Überwachung des gesamten Bauprozesses, die Bereitstellung der nötigen Materialien sowie das Geben von «Hilfestellung und Tipps»(G15-04). "Handwerker» stehen in dieser Sprachfigur für eine «heterogene Gruppe von Lernenden». Der Bauprozess wird als Lernen verstanden, bei dem die Handwerker verschiedene Elemente des Hauses fertigstellen. Grenzen dieser Sprachfigur werden u. a. in «der eher passiven Rolle, die der Lehrende einnimmt» gesehen sowie in der offenen Frage, "was mit Handwerkern/Schülern gemacht werden sollte, welche den Bau des Hauses gar nicht oder nicht in vollem Maße bewältigen» (G15-04). 
Die Sprachfigur «Lehren und Lernen ist SENDEN UND EMPFANGEN»(G15-08) wurde in einer Gruppenmetapher zum Ausdruck gebracht (Anhang B.9). Diese Metaphorik wurde bereits in Form einer Ad-hoc-Metapher formuliert und als Hinweis auf weitere Sprachfiguren skizziert (Kap. 7.4.3.2). Die Gruppenmetapher stellt in diesem Zusammenhang eine Erweiterung und Konkretisierung dar. Dafür belegten die Studierende die eigene Metaphorik mit Verweisen auf ein Kommunikationsmodell, dass Kommunikation als Austausch von Informationen zwischen Sendern und Empfängern modelliert bzw. metaphorisiert. Im Bezug des Modells auf den Unterricht markieren die Studierenden die folgenden zentralen Merkmale: (1.) Lehrende werden als Sender verstanden. Das Lehren entspricht in diesem Zusammenhang dem Senden von Informationen: "In diesen Unterrichtseinheiten werden Sachinhalte vom Lehrenden an den Lernenden vermittelt. An dieser Stelle verdeutlicht sich das Sender (Lehrer) Empfänger (Schüler) Verhältnis» (G15-08). (2.) Lernende werden als Empfänger metaphorisiert und Lernen wird entsprechend als Empfangen verstanden. Ferner wird Lernen mit weiteren Begriffen der Informationsverarbeitung als «Informationen zu verarbeiten, abzuspeichern und wiederzugeben» (G15-08) beschrieben. Grenzen bzw. Schwierigkeiten der Metaphorik wurden nicht thematisiert.

Die Darstellung der Sprachfiguren und metaphorischen Muster der Gruppenmetaphern erfolgte mit dem Ziel, eine zweite strukturierte Antwort auf die Frage zu geben, mit welchen Metaphern Studierende ihre Vorstellungen von Lehren und Lernen beschreiben. Die dargestellten metaphorischen Muster sowie Sprachfiguren basieren auf den Überlegungen studentischer Arbeitsgruppen und wurden im Rahmen des Seminars sowie für die Veröffentlichung der Wikibeiträge als konsensuale Vorstellungen vertreten.

\subsubsection{Zusammenfassung und Relevanz der Ergebnisse}

Die Darstellung der Ausdrucksformen und -phänomene der Ad-hoc-Metaphern erfolgte mit dem Ziel, einen strukturierten Einblick in die empirischen Daten der studentischen Arbeits- und Textprodukte zu eröffnen. Dieser Einblick bildete die empirische Grundlage für die weiterführenden Interpretationen und Aussagen, dass (1.) die Aufgabenbearbeitung für Studierende eine Herausforderung darstellt (Kap. 8.2.1), (2.) die verschiedenen Ausdrucksphänomene Irritations- und Reflexionsanlässe eröffnen (Kap. 8.2.2) und dass (3.) Herausforderungen in der wissenschaftlichen Analyse der Schreibprodukte entstehen (Kap. 8.4).

Die drei bei der Darstellung von Ad-hoc-Metaphern identifizierten Phänomene, (1.) «Routinisierte Metaphern «fremder Bildbereiche», (2.) «Bildbrüche innerhalb des Bildbereichs eine Ad-hoc-Metapher» sowie (3.) «nicht nachvollziehbare Bedeutungsübertragungen», haben die Gemeinsamkeit, dass die formulierten Erläuterungen nicht bzw. nur begrenzt dazu beitragen, dass die zum Ausdruck gebrachten Vorstellungen über Lehren und Lernen intersubjektiv nachvollziehbar rekonstruiert werden 
konnten. Für den praktischen Lehr- und Lernkontext eröffnen sich vielfältige Diskussionsanlässe, welche zu einem gemeinsamen Verständnis sowie einer Erweiterung der pädagogischen Artikulationsfähigkeit führen können (Kap. 8.2). Für die wissenschaftliche Analyse, deren Qualität u. a. an der intersubjektiven Nachvollziehbarkeit der Ergebnisse gemessen wird, stellt sich die Herausforderung, mit dieser Vielfalt an Phänomenen umgehen zu müssen (Kap. 8.4.1).

Die Darstellung der Sprachfiguren und metaphorischen Muster erfolgte mit dem Ziel, eine strukturierte Antwort auf die Frage zu geben, mit welchen Metaphern Studierende ihre Vorstellungen vom Lehren und Lernen beschreiben. Die dargestellten metaphorischen Muster bündeln dabei einzelne Sprachfiguren, die als kohärente Übertragungen von Begriffen eines Herkunftsbereichs auf den Zielbereich des Lehrens und Lernens im Unterricht erfolgen. In Anlehnung an de Guerrero und Villamil (2000, 2002) wird davon ausgegangen, dass es über die Versprachlichung dieser expliziten Metaphern möglich wird, die bewusstseinsfähigen bzw. leicht zugänglichen Vorstellungen über Lehren und Lernen zu artikulieren und die Rekonstruktion der zugrundeliegenden metaphorischen Muster Rückschlüsse auf die Vorstellungswelt der Studierenden erlaubt (Kap. 5.3.3). Dabei zeigt sich eine Vielfalt an Bildbereichen und unterschiedlichen Verständnissen. Auf Basis dieser Ergebnisse eröffnet sich die Möglichkeit, dass das Diskussions- und Reflexionspotenzial der Aufgabenstellung sowie der in diesem Zusammenhang entstandenen Produkte zu diskutieren (Kap. 8.2.2). Aus forschungsmethodischer Sicht eröffnet sich zudem die Möglichkeit, Vergleiche mit den metaphorischen Mustern der Gruppenprodukte (Kap. 7.4.3.4) anzustellen, um perspektivisch die didaktische Sinnhaftigkeit der Gruppenphasen zu diskutieren (Kap. 8.2.3).

Die exemplarische Erläuterung der Darstellungsformen und Strukturelemente der Gruppenmetaphern diente dem Zweck, einen strukturierten Einblick in die empirischen Daten der studentischen Gruppen- bzw. Textprodukte zu eröffnen. Ferner wurden bereits in der formalen Betrachtung der Gruppenprodukte Hinweise sichtbar, in welcher Weise es Studierenden gelang, ihr Denken über Lehren und Lernen zum Ausdruck zu bringen. Die Formulierung von Grenzen einer Metaphorik hinsichtlich des komplexen Zielbereichs des Lehrens und Lernen setzt beispielsweise voraus, dass sich Studierende dieser Komplexität überhaupt bewusst sind. Insofern kann das formale Fehlen einer Grenzbetrachtung als Indikator gedeutet werden, dass diese Voraussetzung nicht erfüllt ist. Auf Basis dieser Daten wird in der Auswertung diskutiert, inwiefern die Aufgabenstellung sowie die in Gruppen formulierten Metaphern dazu beitragen können, die Artikulations- und Reflexionsfähigkeit der Studierenden zu entwickeln bzw. zu erweitern (Kap. 8.2.3). Bei vergleichender Betrachtung der Sprachfiguren und metaphorischen Muster der Ad-hoc-Metaphern zeigen sich dabei (a) Übereinstimmungen, (b) Adaptionen spezifischer Ad-hoc-Metaphern sowie (c) das «Fehlen» von metaphorischen Mustern und Sprachfiguren. Auf Basis dieser 
Ergebnisse eröffnet sich die Möglichkeit, dass das Diskussions- und Reflexionspotenzial der Aufgabenstellung sowie die in diesem Zusammenhang entstandenen Produkte zu diskutieren (Kap. 8.2.3).

\subsubsection{Erkenntnisse der Wikibook-Analyse}

Die Analyse der Versionsgeschichte der im Projekt erstellten Wikiseiten eröffnet verschiedene Einblicke in den Entstehungsprozess des Wikibooks sowie den zugehörigen Interaktionen. Die Versionsgeschichte zeigt Bearbeitungen durch Lehrende, Studierende und Mitglieder der Wikicommunity ausserhalb des institutionellen Seminarkontextes. Als Erweiterung der bisherigen Praxisreflexionen (Kap. 7.2) und der studentischen Perspektiven auf das Wikibook (Kap. 7.4.1, Kap. 7.4.2) können faktisch stattgefundene Interaktionen mit verschiedenen Wikiseiten und Nutzungsweisen des Wikibooks bzw. des Wiki-Editors beschrieben werden. Zur Beantwortung der Fragestellung, welche Erfahrungen (und medienbezogene Reflexionsanlässe) die Gestaltung eines Wikibooks im Kontext der angebotenen Seminare eröffnen können, werden auf Basis der Wikidaten bereits getroffene Aussagen bestätigt und weitere Aussagen formuliert. Die Auswertung und Darstellung der Ergebnisse erfolgen für die einzelnen Phasen des Konzepts, wie folgt:

- Phase I: (Gemeinsames) Verschriftlichen der Gruppenmetaphern (Kap. 7.4.4.1)

- Phase II: (Gemeinsames) Verschriftlichen der Modelle (Kap. 7.4.4.2)

- Phase III: Individuelle Erstellung (und Veröffentlichung) von Analysen (Kap. 7.4.4.3)

Um Aussagen über die kollaborative Verwendung des Wiki-Editors zu treffen, wird die Anzahl an Bearbeiterinnen und Bearbeitern (Editors) einer Seite, die Anzahl an Bearbeitungen (Edits) sowie die Anzahl an Bearbeitungen ohne angemeldeten Account (IP-Edits) dargestellt. Ferner wird angegeben, ob der hinzugefügte Textumfang bei Seitenerstellung dem grössten hinzugefügten Textumfang einer Seitenbearbeitung entspricht $(F E=M E)$. Diese Information bietet einen Hinweis auf die Frage, ob die Texte im Wiki-Editor gemeinsam erstellt oder lediglich veröffentlicht wurden.

\subsubsection{Phase I: (Gemeinsames) Verschriftlichen der Gruppenmetaphern}

Die erste Phase des Seminarkonzeptes umfasste verschiedene Aktivitäten zur Artikulation der eigenen Vorstellungen der Studierenden mithilfe von Metaphern (Kap. 6.6, Kap. 7.2.2). Als Ergebnis sollten die studentischen Arbeitsgruppen ihre gemeinsam entwickelte Metapher in Form eines Wikibeitrages im Rahmen des zweiten Kapitel des Wikibooks bis zum 3. Mai 2015 verschriftlichen. Zur Analyse der Versionsgeschichte der verschiedenen Wikiseiten wurde der Auswertungszeitraum vom 01. April 2015 bis zum 03. Mai 2015 gewählt ${ }^{241}$. Auf Basis dieser Daten wird im Folgenden

241 Der erste April wurde als Semesterbeginn ausgewählt. 
gezeigt, dass verschiedene Personen an der Gestaltung der Startseite mitwirkten und so erste Erfahrungen zur kollaborativen Texterstellung möglich waren (Abb. 7.14). Zugleich wird sichtbar, dass Studierende in der Bearbeitung ihrer inhaltlich zu verantwortenden Wikiseiten den Wikieditor überwiegend nicht zum kollaborativen Schreiben verwendeten (Tab. 7.18). Interessant ist insbesondere die Aktivität von Wikibookianerinnen und Wikibookianern. Diese haben an der Mehrheit der Wikiseiten Bearbeitungen vorgenommen (z. B. Abb. 7.14 und 7.15) und so Möglichkeiten zur partizipativen Gestaltung von Wikiseiten für Studierende potenziell zugänglich gemacht, wie im Folgenden näher erläutert wird.

Die Startseite des Wikibooks «Lehren, Lernen und Bildung metaphorisch verstehen» kann auf Basis der Bearbeitungsstatistiken als zentrale Seite bzw. die Seite des Buchprojektes mit den häufigsten Interaktionen beschrieben werden. Diese zentrale Stellung lässt sich zum einen mit der Anzahl von 15 unterschiedlichen Bearbeiterinnen und Bearbeitern der Seite begründen (Tab. 7.18). Neben Studierenden und Lehrenden des Seminars gehören zu den Bearbeiterinnen und Bearbeitern auch Wikibookianerinnen und Wikibookianern. Zum anderen kann die Anzahl von 48 Bearbeitungen (Tab. 7.18) der Seite als Indikator für die zentrale Stellung gewertet werden, da sie am häufigsten aktualisiert wurde. Zwischen der Erstellung der Seite am 17.04.2015 und dem Ende des Auswertungszeitraumes am 3.05.2015 wurde sie im Mittel 4,7-mal pro Tag bearbeitet. Ferner wurde nur für die Startseite des Wikibooks im Rahmen des ersten Auswertungszeitraumes eine Diskussionsseite erstellt (Tab. 7.18). Die Diskussionsseite wurde von Wikibookianerinnen und Wikibookianern und mir in der Rolle als Lehrender genutzt. 


\begin{tabular}{|c|c|c|c|c|}
\hline & Editors & Edits & IP-Edits & $\mathrm{FE}=\mathrm{ME}$ \\
\hline Start $^{\star}$ & 15 & 48 & $14(29 \%)$ & $\mathrm{Ja}$ \\
\hline Diskussion:Start & 3 & 9 & 0 & $\mathrm{Ja}$ \\
\hline Einleitung & 1 & 1 & 0 & $\mathrm{Ja}$ \\
\hline Metahern und Vorstellungen & \multicolumn{4}{|c|}{ keine Bearbeitungen } \\
\hline \multicolumn{5}{|l|}{ Lehren und Lernen ist wie ... } \\
\hline ...von einem Reisebegleiter begleitet zu werden & \multicolumn{4}{|c|}{ keine Bearbeitungen } \\
\hline ...Wandern* & 3 & 5 & $2(40 \%)$ & $\mathrm{Ja}$ \\
\hline ...von einem Reiseführer begleitet werden & 1 & 2 & 0 & $\mathrm{Ja}$ \\
\hline ...Häuser bauen ohne konkreten Bauplan & 1 & 2 & $2(100 \%)$ & $\mathrm{Ja}$ \\
\hline ...der Garten des Lebens & 3 & 5 & $4(80 \%)$ & $\mathrm{Ja}$ \\
\hline ...Gärtnern und Gedeihen & 2 & 2 & $1(50 \%)$ & $\mathrm{Ja}$ \\
\hline ...ein Besuch in einer Bäckerei & 1 & 2 & $2(100 \%)$ & $\mathrm{Ja}$ \\
\hline ...Senden und Empfangen & 9 & 16 & $3(18 \%)$ & $\mathrm{Ja}$ \\
\hline \multicolumn{5}{|l|}{ Bildung ist wie ... } \\
\hline ...ein Garten mit verschiedenen Pflanzen & 2 & 2 & 0 & $\mathrm{Ja}$ \\
\hline ...das Meer & 2 & 5 & $4(80 \%)$ & Nein \\
\hline ...nicht endendes Buch zum Weiterschreiben & 2 & 2 & 0 & $\mathrm{Ja}$ \\
\hline ...eine nie endende Abenteuerreise & \multicolumn{4}{|c|}{ keine Bearbeitungen } \\
\hline
\end{tabular}

Tab. 7.18: Bearbeitungsstatistiken relevanter Wikiseiten der ersten Seminarphase im Auswertungszeitraum vom 17.04.2015 bis 3.05.2015.

Bei näherer Betrachtung der Bearbeiterinnen und Bearbeitern und der Bearbeitungen der Startseite lassen sich Unterschiede zwischen Studierenden, Lehrenden und Wikibookianerinnen und Wikibookianern markieren. Die Diagramme zur Anzahl der Bearbeitungen sowie des hinzugefügten Textes machen zunächst deutlich, dass ich als Lehrender sehr aktiv in die Erstellung des Wikibookprojektes involviert war. Mit etwa $45 \%$ wurde fast die Hälfte der Veränderungen von mir vorgenommen und zugleich stammt über Dreiviertel des hinzugefügten Textes von mir als Lehrender (Abb. 7.14). Hinsichtlich der Aktivität der Studierenden zeigt sich an der hohen Anzahl an IP-Edits, dass diese fast überwiegend unangemeldet die Startseite bearbeiteten. Von den angemeldeten Bearbeiterinnen und Bearbeitern war lediglich ein Account von Studierenden. Die weiteren Bearbeitungen wurden jeweils durch IP-Adressen dokumentiert, wie die Bearbeitungsstatistiken (Tab. 7.18) und Diagramme der Bearbeiterinnen und Bearbeiter (Abb. 7.14) zeigen. 

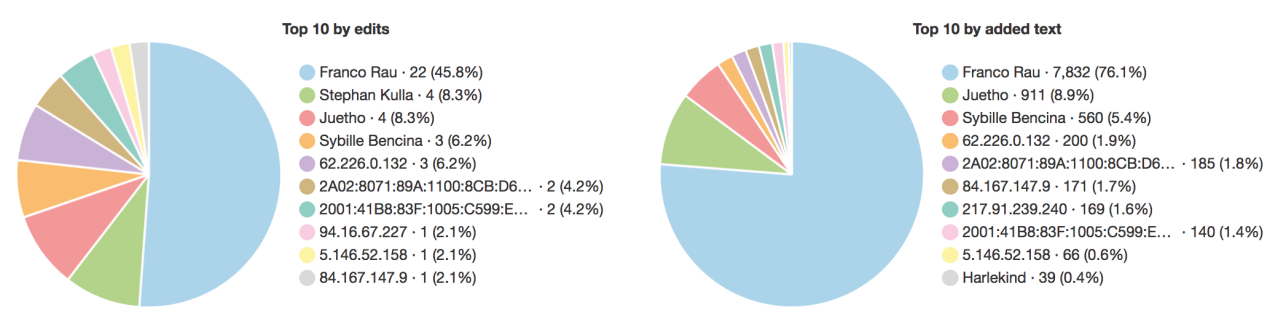

Abb. 7.14: Bearbeitungsanzahl (links) und -umfang (rechts) der zehn aktivsten Bearbeiterinnen und Bearbeiter der Startseite im Zeitraum vom 01.04.2015 bis 03.05.2015.

Hinsichtlich der Zielstellung des Seminars, einen Beitrag für partizipative Kultur in Form eines Wikibooks zu leisten (Kap. 6.2), ist es interessant, dass im Rahmen der ersten Seminarphase bereits Personen am Wikibook mitgearbeitet haben, welche nicht zum Seminar gehörten. Die weiterführenden Statistiken zeigen die Accounts von Juetho und Stephan Kulla mit jeweils vier Bearbeitungen im Auswertungszeitraum. Neben der Bearbeitung von Formatierungs- und Verlinkungsproblemen fügte Juetho zudem am zweitmeisten Text zur Startseite hinzu. Der hinzugefügte Text entspricht der bereits beschriebenen Anleitung für Studierende (Kap. 7.2.2.3). Neben den bereits in der Erprobung beschriebenen Beiträgen zur Diskussionsseite (Kap. 7.2.2.3) wird bei Betrachtung der Bearbeiterinnen und Bearbeitern der studentischen Seiten zudem sichtbar, dass Juetho bei sieben von zehn Beiträgen Bearbeitungen vornahm. Dazu gehören alle studentischen Wikibeiträge im zweiten Kapitel mit mehr als einer bearbeitenden Person (Tab. 7.18). Dabei beschränkten sich die Bearbeitungen überwiegend auf Textformatierung und Korrekturen in der Rechtschreibung. Inhaltliche Überarbeitungen wurden nicht vorgenommen, wie sich auch an der exemplarischen Auswertung der Bearbeiterinnen und Bearbeitern der Seite «Lehren und Lernen ist wie Wandern» zeigt (Abb.7.15). Juetho nahm zwar die häufigsten Bearbeitungen der Seite vor, ergänzte aber keinen inhaltlichen Text.
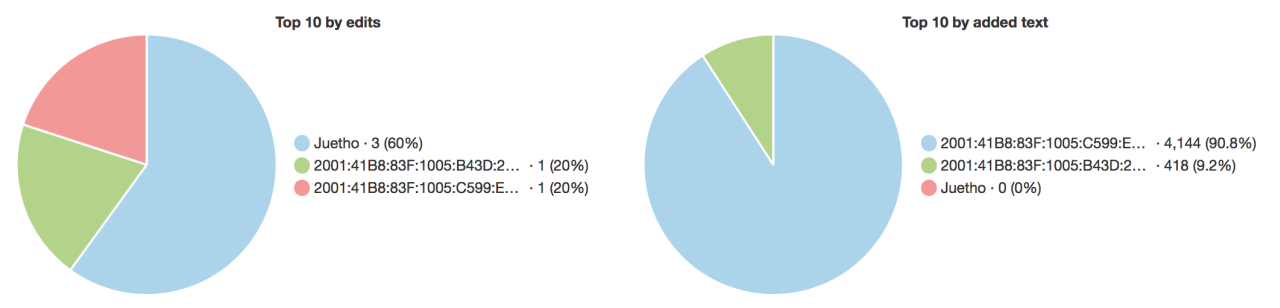

Abb. 7.15: Bearbeitungsanzahl (links) und -umfang (rechts) der zehn aktivsten Bearbeiterinnen und Bearbeiter der Seite «Lehren und Lernen ist wie Wandern» im Zeitraum vom 01.04.2015 bis 03.05.2015.

Die Bearbeitungsstatistiken der Wikiseiten des zweiten Kapitels bieten zudem weitere Informationen über den Umgang der Studierenden mit der Aufgabe, ihre Ergebnisse als Wikibeitrag zu dokumentieren (Tab. 7.18). Die geringe Anzahl an 
Editoren und an Bearbeitungen können als Indikator dafür gewertet werden, dass die Texte kaum kollaborativ über die Möglichkeiten des Wikis geschrieben wurden. Die Information, dass bei neun von zehn Wikibeiträgen der erste Beitrag jeweils den umfangreichsten Beitrag darstellt, stützt diese These. Ferner wird durch die hohe Anzahl an IP-Edits deutlich, dass die Mehrheit der Studierenden unangemeldet Veränderungen vornahm. Die exemplarische Auswertung der Seite «Lehren und Lernen ist wie Wandern» (Abb.7.15) veranschaulicht diese Aussagen. Von zwei unangemeldeten Personen wurde jeweils durch eine Bearbeitung Text hinzugefügt. Formale Korrekturen wurden vom angemeldeten Wikibookianer Juetho vorgenommen.

\subsubsection{Phase II: (Gemeinsames) Verschriftlichen der Modelle}

Die zweite Phase des Seminarkonzeptes widmete sich der Erarbeitung didaktischer Modelle sowie Lern- bzw. Bildungstheorien (Kap. 6.6, 7.2.3). Die studentischen Arbeitsgruppen wurden aufgefordert, ihre Auseinandersetzung mit dem jeweils gewählten Modell bzw. der gewählten Theorie als Wikibeitrag im dritten Kapitel des Wikibooks zu verschriftlichen. Während die Studierenden die Möglichkeit hatten, Zwischenstände in einer geschützten Umgebung über Moodle und in der Präsenz zur Diskussion zu stellen, sollte die vorläufige Endfassung bis zum 23. Juni ausformuliert sein. Die Analyse der Versionsgeschichte erfolgte entsprechend im Zeitraum vom 04. Mai 2015 bis zum 23. Juni 2015242. Dabei zeigt sich erneut, dass die Startseite des Projektes von verschiedenen Menschen gestaltet wurde und kollaborative Erfahrungen möglich waren. Bei der Betrachtung spezifischer Wikiseiten der Studierenden zu erziehungswissenschaftlichen Denkwerkzeugen wurde erneut deutlich, dass Studierende den Wiki-Editor überwiegend zur Veröffentlichung ihrer Arbeitsergebnisse verwendeten und nur selten die Möglichkeit zur kollaborativen Texterstellung nutzten. Ferner hatten sich Wikibookianerinnen und Wikibookianer an der Bearbeitung der verschiedenen Wikiseiten beteiligt, wie im Folgenden näher erläutert wird.

Auf Basis der Bearbeitungsstatistiken (Tab. 7.19) kann die Startseite des Wikibooks auch in dieser Phase als zentrale Seite bzw. als Seite mit den häufigsten Interaktionen beschrieben werden. Im Auswertungszeitraum zwischen Mai und Juni haben zum einen 13 unterschiedliche Bearbeiterinnen und Bearbeiter die Seite verändert. Zu den Bearbeiterinnen und Bearbeitern gehörten Studierende, Lehrende und Personen der Wikibook-Community (Abb. 7.16). Die Anzahl von 23 Bearbeitungen (Tab. 7.19) kann erneut als Indikator für die zentrale Stellung gewertet werden. Im Vergleich zur ersten Phase fällt die Häufigkeit der Bearbeitungen mit 0,6 Bearbeitungen pro Tag in der zweiten Phase im Mittel deutlich geringer aus. Neben den inhaltlichen und formalen Änderungen der Startseite wurde zudem die Diskussionsseite der Startseite verwendet.

242 Der Auswertungszeitraum wurde als Zeitraum zwischen den zwei Abgabeterminen gewählt. 


\begin{tabular}{|c|c|c|c|c|}
\hline & Editors & Edits & IP-Edits & $\mathrm{FE}=\mathrm{ME}$ \\
\hline Start* & 13 & 23 & $11(48 \%)$ & - \\
\hline Diskussion:Start & 1 & 4 & 0 & - \\
\hline Erziehungswissenschaftliche Denkwerkzeuge & \multicolumn{4}{|c|}{ keine Bearbeitungen } \\
\hline \multicolumn{5}{|l|}{ Didaktische Modelle } \\
\hline Bildungstheoretische Didaktik & 5 & 7 & $3(43 \%)$ & $\mathrm{Ja}$ \\
\hline Kommunikative Didaktik & 3 & 8 & $1(13 \%)$ & $\mathrm{Ja}$ \\
\hline Lehr-/Lerntheoretische Didaktik & \multicolumn{4}{|c|}{ keine Bearbeitungen } \\
\hline \multicolumn{5}{|l|}{ Lerntheorien } \\
\hline Konstruktivismus & 4 & 12 & $4(33 \%)$ & Ja \\
\hline Behaviorismus & 3 & 4 & 0 & $\mathrm{Ja}$ \\
\hline Kognitivismus & 5 & 28 & $17(61 \%)$ & $\mathrm{Ja}$ \\
\hline Neurowissenschaften & 4 & 7 & $5(71 \%)$ & $\mathrm{Ja}$ \\
\hline \multicolumn{5}{|l|}{ Bildungstheorien } \\
\hline Formale Bildungstheorie & 2 & 5 & 0 & $\mathrm{Ja}$ \\
\hline Kategoriale Bildung & \multicolumn{4}{|c|}{ keine Bearbeitugen } \\
\hline Theorie der Halbbildung & 6 & 8 & $6(75 \%)$ & Ja \\
\hline Theorie der materialen Bildung & 2 & 2 & $1(50 \%)$ & $\mathrm{Ja}$ \\
\hline
\end{tabular}

Tab. 7.19: Bearbeitungsstatistiken relevanter Wikiseiten der zweiten Seminarphase im Auswertungszeitraum vom 04.05.2015 bis 23.06.2015.

Die zugehörige Statistik der Bearbeiterinnen und Bearbeiter zeigt, dass Studierende, Lehrende und Mitglieder der Wikicommunity erneut Bearbeitungen auf der Startseite vornahmen (Abb. 7.16). Zum anderen werden Unterschiede im Vergleich zur ersten Seminarphase erkennbar. Dazu gehört u. a., dass Anzahl und Umfang an Bearbeitungen durch mich als Lehrenden deutlich geringer sind als in der ersten Phase. Meine Veränderungen beschränkten sich auf die Anpassung der Anleitung zur Erstellung neuer Wikiseiten sowie die Erweiterung des Inhaltsverzeichnisses. Bei den Studierenden zeigt sich eine Veränderung hinsichtlich der Verwendung von Accounts. So übernahmen in dieser Phase fünf Studierende als angemeldete Nutzerinnen und Nutzer das Editieren, während sechs Studierende unangemeldet Veränderungen an der Startseite vornahmen. Zudem zeigt das Tortendiagramm zu den zehn aktivsten Bearbeiterinnen und Bearbeiter, dass sich deren Aktivität deutlich gleichmässiger verteilt (Abb. 7.16). 

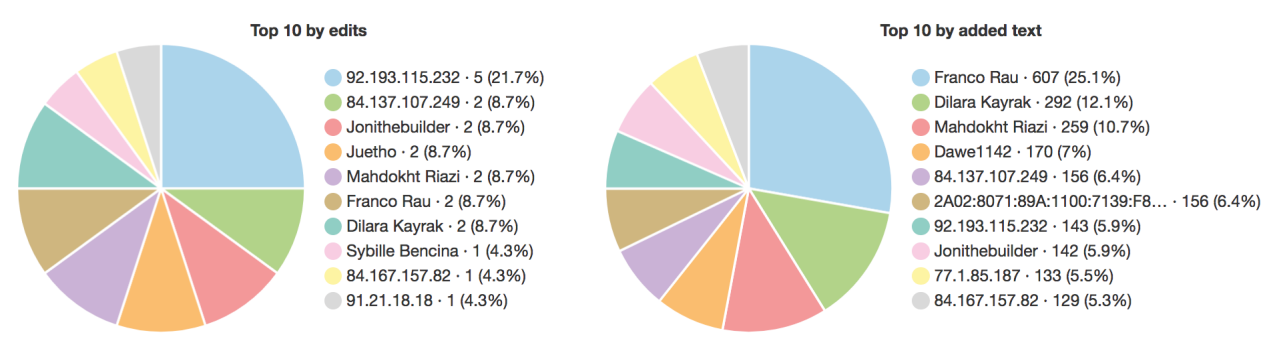

Abb. 7.16: Bearbeitungsanzahl (links) und -umfang (rechts) der zehn aktivsten Bearbeiterinnen und Bearbeiter der Startseite im Zeitraum vom 04.05.2015 bis 23.06.2015.

Unter den aktiven Bearbeiterinnen und Bearbeitern finden sich auch in der zweiten Seminarphase Mitglieder der Wikicommunity. Dies zeigt sich hinsichtlich der Bearbeitung der Startseite (Abb. 7.16), weiterer Beiträge auf der zugehörigen Diskussionsseite sowie verschiedener Bearbeitungen auf den Wikiseiten der Studierenden (Abb. 7.17). Die Diskussionsseite der Startseite wurde in dieser Phase ausschliesslich von einem Mitglied der Wikicommunity ergänzt. Die Bearbeitungen auf der Startseite sowie den inhaltlichen Seiten umfassten vor allem Überarbeitungen formaler Probleme, z. B. unangemessene Verlinkungen oder Formatierungen. Bemerkenswert ist dabei, dass auf allen neun von Studierenden angelegten Seiten auch Bearbeitungen durch mindestens einem Mitglied der Wikicommunity vorgenommen wurden. Dies zeigt sich exemplarisch auf der Wikiseite «Konstruktivismus» (Abb. 7.17). Interessant ist ebenfalls, dass auf den Wikiseiten zum Behaviorismus, zum Kognitivismus sowie zu den Neurowissenschaften jeweils zwei angemeldete Wikibookianer aktiv waren (und nur eine angemeldete Person). Eine inhaltliche Auseinandersetzung zu einem Wikibeitrag fand nur zu einer Wikiseite "Theorie der Halbbildung» statt. Zu diesem Beitrag wurde eine Diskussionsseite erstellt, auf welcher Aussagen des Beitrages hinterfragt wurden. Die Bearbeitung der Diskussionsseite erfolgte ohne Angabe eines Accounts.
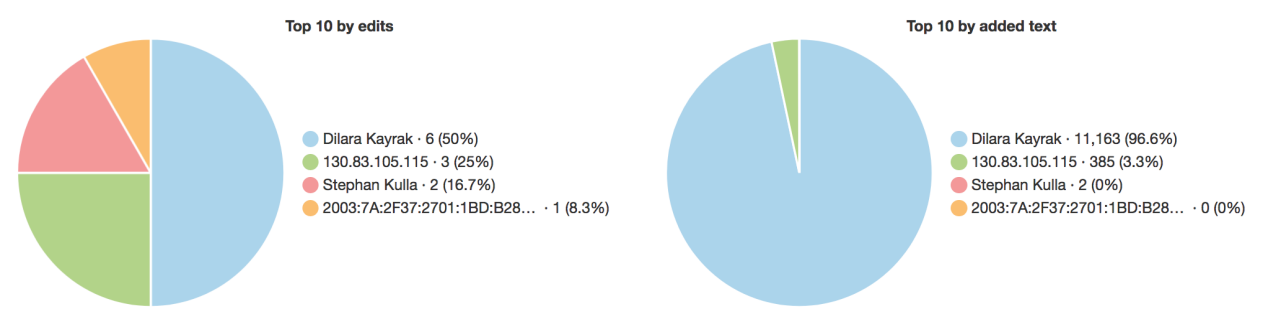

Abb. 7.17: Bearbeitungsanzahl (links) und -umfang (rechts) der zehn aktivsten Bearbeiterinnen und Bearbeiter der Wikiseite «Konstruktivismus» im Zeitraum vom 04.05.2015 bis 23.06.2015. 
Die Statistiken zur Bearbeitung der Wikiseiten der erziehungswissenschaftlichen Denkwerkzeuge bieten Indikatoren für Aussagen zur (ausgebliebenen) wiki-basierten Kollaboration (Tab. 7.19). Das Ergebnis, dass bei allen Wikiseiten der erste Beitrag vom Umfang her auch dem grössten Beitrag entspricht, bietet einen empirischen Indikator dafür, dass das Wikibook vor allem zur Veröffentlichung der Arbeitsergebnisse genutzt wurde. Die einstellige Anzahl an Bearbeitungen ${ }^{243}$ bei sieben von neun Beiträgen kann als weiterer Indikator dafür gedeutet werden, dass die Möglichkeiten zum kollaborativen Schreiben nur von wenigen Seminarteilnehmerinnen und -teilnehmern genutzt wurden. Die maximale Anzahl von jeweils drei angemeldeten Bearbeiterinnen und Bearbeiter ${ }^{244}$, von denen häufig zwei Wikibookianer waren, deutet darauf hin, dass sich in den Gruppen nur wenige Personen aktiv mit der Gestaltung der Wikiseiten auseinandergesetzt haben. Dies zeigt sich auch exemplarisch an der Wikiseite «Konstruktivismus» (Abb. 7.17). Mit 12 Bearbeitungen ist diese studentische Wikiseite einerseits die am zweithäufigsten bearbeitete Seite der Seminarphase (Tab. 7.19) Anderseits hat nur eine Person etwa $96 \%$ des gesamten Textes zu dieser Wikiseite hinzugefügt. Dies kann als weiterer Hinweis darauf gedeutet werden, dass die Auseinandersetzung mit dem Wikibook in den meisten Gruppen von einem «WikiVerantwortlichen» übernommen wurde.

\subsubsection{Phase III: Individuelle Erstellung (und Veröffentlichung) von Analysen}

Die dritte Phase des Seminarkonzeptes widmete sich der theoriegeleiteten Analyse und Reflexion der eigenen Metaphern (Kap. 6.6, 7.2.4). Studierende hatte im Rahmen dieser Seminarphase individuell die Aufgabe, die entwickelte Gruppenmetapher hinsichtlich einer selbstgewählten Fragestellung mithilfe der erarbeiteten erziehungswissenschaftlichen Denkwerkzeuge zu analysieren und dies zu verschriftlichen. Entgegen der ursprünglichen Planung wurden die Studierenden nicht verpflichtet, ihre Texte auf dem Wikibook zu veröffentlichen (Kap. 7.2.4.3). Stattdessen hatten sie zum Abschluss des Seminars die freiwillige Möglichkeit zur Veröffentlichung ihrer Analysen und Reflexionen im Wikibook. Die Analyse der Versionsgeschichte erfolgte im Zeitraum vom 24. Juni 2015 bis zum 30. September $2015^{245}$. Wie in den vorherigen Phasen zeigt sich, dass die Startseite des Projektes von verschiedenen Menschen gestaltet wurde und kollaborative Erfahrungen in Ansätzen möglich waren. Die Möglichkeit für Studierende, die vorbereiten Wikiseiten zur Veröffentlichung der eigenen

243 Relativ klein meint an dieser Stelle weniger als zehn Bearbeitungen.

244 Dies wird in den Tabellen nicht sichtbar, weil IP-Edits von unterschiedlichen IPs jeweils als einzelne Autorinnen und Autoren gewertet werden. Viele Editoren bei gleichzeitig vielen IP-Edits deuten darauf hin, dass eine Person (oder mehrere) unangemeldet mit verschiedenen IPs bzw. von verschiedenen Standorten oder zu verschiedenen Zeiten mitgearbeitet haben. Dies führt beispielsweise auch zu dem Artefakt, dass bei der Theorie der Halbbildung mehr Editoren mitgewirkt haben als Gruppenmitglieder und aktive Wikibookianerinnen und Wikibookianern vorhanden waren.

245 Der Auswertungszeitraum wurde als Zeitraum zwischen den zwei Abgabeterminen gewählt. 
Beiträge zu verwenden, wurde nur von wenigen Studierenden genutzt. Gleichwohl waren verschiedene Studierende aktiv in die Überarbeitung vorheriger Beiträge involviert. Wikibookianerinnen und Wikibookianern haben sich ebenfalls im Zeitraum der dritten Seminarphase eingebracht, wie im Folgenden exemplarisch gezeigt wird.

Die Startseite des Wikibooks «Lehren, Lernen und Bildung metaphorisch verstehen» wurde in der dritten Phase von zehn unterschiedlichen Bearbeiterinnen und Bearbeiter insgesamt 36-mal bearbeitet (Tab. 7.20). Zu den Bearbeiterinnen und Bearbeiter gehörten Studierende, Lehrende und Mitglieder der Wikicommunity. Die zentralen Überarbeitungen der Startseite umfassten in dieser Seminarphase die Neugestaltung des Inhaltsverzeichnisses auf Basis der Abstimmung der Studierenden (Kap. 7.2.3.3). Zudem wurden fehlende Wikiseiten ergänzt und formale Fehler behoben.

\begin{tabular}{|l|l|l|l|l|}
\hline & Editors & Edits & IP-Edits & FE = ME \\
\hline Start* & 10 & 36 & $10(28 \%)$ & - \\
\hline Diskussion:Start & 3 & 14 & 0 & - \\
\hline Analysen und Reflexionen & 2 & 5 & 0 & Ja \\
\hline$\ldots$ aus didaktischer Perspektive & 5 & 9 & $4(44 \%)$ & Nein \\
\hline ... aus lerntheoretischer Perspektive & 3 & 3 & $1(33 \%)$ & Nein \\
\hline ... aus bildungstheoretischer Perspektive & 5 & 8 & $2(25 \%)$ & Nein \\
\hline
\end{tabular}

Tab. 7.20: Bearbeitungsstatistiken relevanter Wikiseiten der dritten Seminarphase im Auswertungszeitraum vom 24.06.2015 bis 30.09.2015.

Die Diagramme zur Aktivität der Bearbeiterinnen und Bearbeiter zeigt, dass viele Veränderungen von Lehrenden vorgenommen wurden (Abb. 7.18). Neben der Aktualisierung des Inhaltsverzeichnisses umfassten die Bearbeitungen das Anlegen neuer Seiten zum neuen Kapitel in der dritten Seminarphase sowie das Ergänzen bereits bestehender Platzhalter mit Wikiseiten zu den jeweiligen Kapiteleinleitungen. Das Editieren der Startseite von Studierenden beinhaltete das Hinzufügen bisher noch offener Wikiseiten zu den Gruppenmetaphern und erziehungswissenschaftlichen Denkwerkzeugen. Interessant ist, dass die Überarbeitung verschiedener Wikibeiträge zu vielfältigen Interaktionen führte. Im Vergleich zu den vorherigen Phasen wurde in diesem Auswertungszeitraum z. B. die Seite «Kommunikative Didaktik» häufiger bearbeitet als die Startseite. Die Seite wurde 46-mal editiert von formal zehn unterschiedlichen Bearbeiterinnen und Bearbeitern ${ }^{246}$. Diskussionsbeiträge beschränkten sich in dieser Phase erneut auf Beiträge zur Diskussion der Startseite bzw. des gesamten Projektes. Beiträge zur Diskussionsseite erfolgten nur von Mitgliedern der Wikicommunity und Lehrenden.

246 Aufgrund der hohen Anzahl von Bearbeitungen ohne angemeldeten Account (38 bzw. 82.6\%) kann an dieser Stelle nicht gesagt werden, inwiefern es sich wirklich um zehn unterschiedliche Personen handelte. 

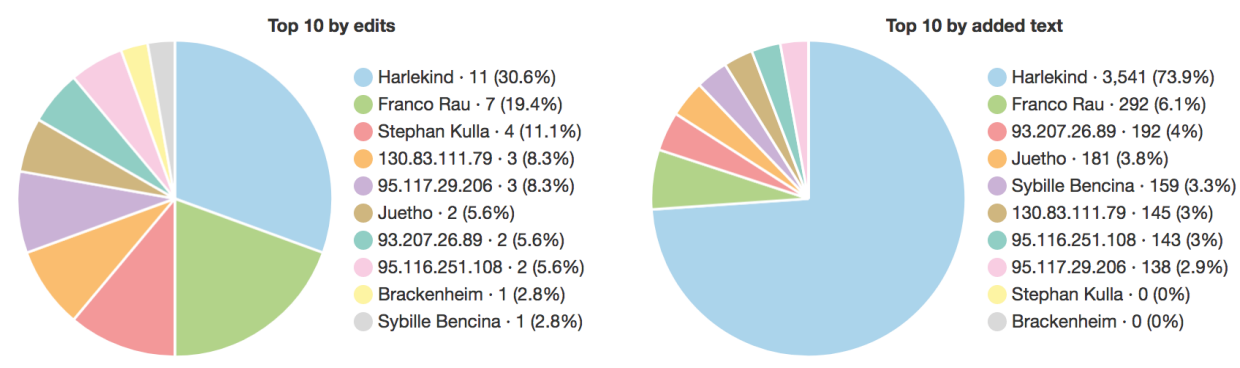

Abb. 7.18: Bearbeitungsanzahl (links) und -umfang (rechts) der zehn aktivsten Bearbeiterinnen und Bearbeiter der Startseite im Zeitraum vom 24.06.2015 bis 30.09.2015.

Die Aktivität der Wikibookianer (Juetho und Stephan Kulla) bestand, wie in den vorherigen Phasen, in Form von Überarbeitungen formaler Probleme auf der Startseite (Abb. 7.18), die Erstellung neuer Beiträge zur Diskussionsseite sowie in Überarbeitungen auf den verschiedenen Wikiseiten, welche in der dritten Seminarphase entstanden waren. Stephan Kulla hatte mit mehreren Bearbeitungen an allen Wikiseiten zu den Analysen und Reflexionen aus didaktischer, lerntheoretischen und bildungstheoretischer Perspektive mitgeschrieben und stellte entsprechend jeweils einen angemeldeten Editor dar (z. B. Tab. 7.20).
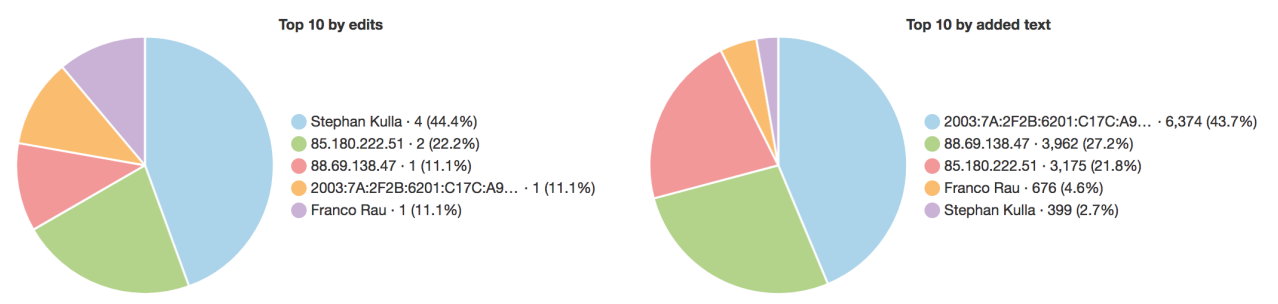

Abb. 7.19: Bearbeitungsanzahl (links) und -umfang (rechts) der zehn aktivsten Bearbeiterinnen und Bearbeiter der Seite «Analysen und Reflexionen aus didaktischer Perspektive» im Zeitraum vom 24.06.2015 bis 30.09.2015.

Die Bearbeitungen der Studierenden beschränkten sich im vierten Kapitel des Wikibooks auf insgesamt acht Bearbeitungen, davon sieben inhaltliche Beiträge bzw. hochgeladenen Analysen und Reflexionen. Nur eine Bearbeitung erfolgte mit einem angemeldeten Account. Im Vergleich zu den insgesamt 39 verschiedenen Analysen und Reflexionen, welche in den Moodle-Foren veröffentlicht wurden, stellen die sieben Beiträge im Wikibook nur einen geringen Anteil dar (18\%). Das freiwillige Veröffentlichen hatten nur wenige Studierende als Erfahrungsmöglichkeit zur Gestaltung eigener Wikibeiträge wahrgenommen. Jedoch erfolgten auf den Wikiseiten der vorherigen Seminarphasen vielfältige Überarbeitungen. 


\subsubsection{Zusammenfassung und Relevanz Analyse der Versionsgeschichte}

Die Analyse der Versionsgeschichte erfolgte mit dem Ziel, faktische Aussagen über die gestaltungsorientierte Nutzung des Wikibooks durch Studierende, Lehrende und Wikibookianerinnen und Wikibookianern zu treffen. In Ergänzung der bisherigen Praxisreflexionen (Kap. 7.2) und studentischen Beschreibungen zum Umgang mit dem Wikibook (Kap. 7.4.2, 7.4.1) wurde so versucht, weitere Erkenntnisse zu potenziellen Lernsituationen zur Teilhabe an öffentlichen Wikis (Kap. 3.2.1.2) zu gewinnen. Ferner lassen sich über die Versionsgeschichte Hinweise auf die Schreibstrategien der Studierenden im Umgang mit dem Wikibook identifizieren und so Erkenntnisse zur übergeordneten Fragestellung Erfahrungsmöglichkeiten zum Lernen mit sozialen Medien (Kap. 3.2.1.3) formulieren. Die Darstellung der Analyse der Versionsgeschichte erfolgte für die drei gewählten Seminarphasen sowie hinsichtlich der jeweils relevanten Wikibeitäge wie folgt: Phase I: (Gemeinsames) Verschriftlichen der Gruppenmetaphern (Kap. 7.4.4.1), Phase II: (Gemeinsames) Verschriftlichen der Modelle (Kap. 7.4.4.2), Phase III: Individuelle Erstellung (und Veröffentlichung) von Analysen (Kap. 7.4.4.3).

Dabei stützen die Ergebnisse der Wikibookanalyse die Beschreibungen der Studierenden zum Umgang mit dem Wikibook. Die Mehrheit der studentischen Arbeitsgruppen nutzen Wikibooks vor allem zum «Hochladen» bzw. veröffentlichten ihrer Ergebnisse (Kap. 7.4.1.3). Die Möglichkeiten zur kollaborativen Texterstellung wurde von den Studierenden kaum verwendet. Zudem wird sichtbar, dass verschiedene Wikiseiten, wie z. B. die jeweiligen Kapiteleinführungen nicht rechtzeitig in der jeweiligen Seminarphase formuliert wurden. Ferner konnte gezeigt werden, dass die Wikibookianerinnen und Wikibookianern nicht nur Kommentare auf den Diskussionsseiten formulierten (Kap. 7.2.2.3), sondern die Wikiseiten der Studierenden in Form von formalen Korrekturen überarbeiteten. Entsprechende Erfahrungen wurden von den Studierenden in den Interviews (Kap. 7.4.1.4) sowie in den Gruppengesprächen nicht thematisiert (Kap. 7.4.2.2.2).

Relevante Aspekte der Wikibookanalyse werden im folgenden Kapitel «Auswertung und Neuperspektivierung» (Kap. 8) aufgegriffen und zur Modifikation des Entwurfs diskutiert. Beim Vergleich mit den Ergebnissen der Interviews (Kap. 7.4.1) sowie der Veranstaltungsevaluation (Kap. 7.4.2) werden die Ergebnisse der Wikibookanalyse u. a. hinsichtlich zweier Aspekte diskutiert (a) Mitgestaltung eines Wikibooks als Erfahrungs- und Reflexionsmöglichkeit (Kap. 8.3) sowie (b) Wikibook als offenes und veränderbares Lehr- und Lernmaterial (Kap. 9.2.3). 


\subsection{Zusammenfassung}

Das vorliegende Kapitel dokumentierte die Forschungsphase der experimentellen Praxis der ersten Iteration im Sommersemester 2015. Die empirische Analyse untersuchte die übergeordnete Fragestellung: inwiefern die Mitgestaltung eines öffentlichen Wikibooks im Rahmen erziehungswissenschaftlicher Seminare Anlässe schaffen kann, um über das Lernen mit Medien die Entwicklung einer pädagogischen Artikulations- und Reflexionsfähigkeit zu fördern und das Lernen über Medien anzuregen (Kap. 3.2). Die empirische Erhebung und Analyse erfolgte in Form eines multiperspektivischen Vorgehens (Kap. 3.2.2).

Auf Basis der Praxisreflexion kann akzentuierend hervorgehoben werden, dass sich insbesondere im Rahmen des ersten Präsenztermins zeigte, dass die Formulierung von Ad-hoc-Metaphern sowie die gemeinsame Entwicklung von Gruppenmetaphern zu vielfältigen Diskussionsanlässen führte (Kap. 7.2.2.1). Die Realisierung des Wikibooks eröffnete durch öffentliche Interaktionen mit Wikibookianerinnen und Wikibookianer interessante Diskussions- und Reflexionsanlässe zur Wissensproduktion in öffentlichen Wikigemeinschaften (Kap. 7.2.2.3, 7.2.3.3). Zugleich wurde die Mitgestaltung eines öffentlichen Wikibooks als Herausforderung (für Studierende und Lehrende) erlebt und führte zu einer Entwurfsadaption in der dritten Seminarphase (Kap. 7.2.3.1, 7.2.4).

In Rahmen einer strukturierenden Inhaltsanalyse von elf problemzentrierten bzw. episodischen Interviews zeigte sich u. a., dass mehrere Studierende die Projektidee positiv bewerteten. Es bestätigte sich die Annahme zu den Lernvoraussetzungen, dass die Mehrheit der Studierenden kaum über Erfahrungen bezüglich einer produktiven Teilhabe an öffentlichen Sharing-Communities verfügte. Die Nutzung der Möglichkeiten zur kooperativen oder kollaborativen Zusammenarbeit über das Wikibook wurden kaum von Studierenden berichtet. Vielmehr wurde das Wikibook als Veröffentlichungsplattform wahrgenommen, welches in mehreren studentischen Arbeitsgruppen nur von einzelnen Personen in produktiver Weise mitgestaltet wurde. Die Interaktionen mit den Mitgliedern der Wikicommunity wurden von verschiedenen Studierenden wahrgenommen und mit unterschiedlichen Schwerpunkten reflektiert.

Auf Basis der quantitativen Veranstaltungsevaluation zeigte sich u. a. eine positive Bewertung des gesamten Seminars sowie der Seminarstruktur (Kap. 7.4.2.1.1). Die Durchführung des Wikibookprojektes ermöglichte der Mehrheit der Studierenden neuen Erfahrungen mit digitalen Medien, welche zugleich sehr unterschiedlich bewertet wurden (Kap. 7.4.2.1.2). Problematisiert wurde die Aktivität der Gruppenteilnehmerinnen und -teilnehmer sowie das Vorgehen zur Gruppenbildung (Kap. 7.4.2.1.2). Die zusammenfassende Inhaltsanalyse der Gruppengespräche offenbarte verschiedene Erkenntnisse zur Beantwortung der Fragestellung, inwiefern das Seminarkonzept neue Erfahrungen zum Lernen mit Medien ermöglichte. Dies zeigte sich 
u. a. daran, dass die Studierenden die Originalität und Innovation des Seminarkonzeptes positiv hervorhoben (Kap. 7.4.2.2.1) und sich eine intensive Thematisierung der Potenziale digitaler Medien für den Schulunterricht wünschten (Kap. 7.4.2.2.3). Die erhalten Rückmeldungen zu den erstellten Arbeitsprodukten wurden von den Studierenden als hilfreich und nachvollziehbar bewertet (Kap. 7.4.2.2.4).

Mithilfe einer Metaphernanalyse der studentischen Arbeitsprodukte wurden Erkenntnisse zur Beantwortung der Frage gewonnen, inwiefern die Formulierung expliziter Metaphern durch Studierende potenzielle Diskussions- und Reflexionsanlässe über Lehren und Lernen eröffnen kann (Kap. 3.2.1.1). Im Fokus der Analyse standen die in der ersten Seminarphase individuell formulierten Ad-hoc-Metaphern sowie die im Wikibook veröffentlichten Gruppenmetaphern. Es wurden 22 Ad-hoc-Metaphern und acht Gruppenmetaphern zum Lehren und Lernen berücksichtigt. Ausgehend von der Annahme, dass es über die Formulierung expliziter Vergleiche und Metaphern möglich wird, die bewusstseinsfähigen bzw. sprachlich leicht zugänglichen Vorstellungen über Lehren und Lernen zu artikulieren (de Guerrero und Villamil 2000, 2002), eröffnete die Analyse der studentischen Produkte einen Einblick in die Ausdrucksfähigkeit sowie in die Vorstellungswelt der Studierenden (Kap. 7.4.3). Exemplarisch hervorgehoben kann an dieser Stelle die in den Ad-hoc-Metaphern sichtbar gewordene Vielfalt an verwendeten Bildbereichen, welche sich in den folgenden vier metaphorischen Mustern zusammenfassen lassen: Lernen und Lehren ist eine SICHTBARE FORTBEWEGUNG NACH PLAN, Lehren ist als MATERIALBEARBEITUNG UND OBJEKTBEWEGUNG, Lehren und Lernen ist GARTENARBEIT UND WACHSTUM sowie Lehren und Lernen ist GEBEN UND NEHMEN (Kap. 7.4.3.2). Im Vergleich dieser Muster konnten unterschiedliche Aspekte und Vorstellungen von Lehren und Lernen gezeigt werden.

Mithilfe der statistischen Auswertung der Versionsgeschichte des Wikibooks konnten Aussagen über die produktive Nutzung des Wikibooks durch Studierende, Lehrende und Mitgliedern der Wikicommunity getroffen werden. Diese Erkenntnisse bieten weitere Hinweise zur Beantwortung der Fragen, inwiefern die Mitgestaltung eines öffentlichen Wikibooks im Kontext eines Seminars Anlässe zur Entwicklung der Fähigkeit zur Teilhabe an partizipativen Medienkulturen eröffnet hat. Im Zeitraum des Sommersemesters wurden 34 Wikiseiten für Wikibook «Lehren, Lernen und Bildung metaphorisch verstehen» erstellt und deren Versionsgeschichte analysiert. Für die ersten zwei Phasen zeigte sich, dass in beiden Seminaren nur wenige Wikiseiten durch kollaborative Schreibprozesse mit dem Wiki-Editor entstanden sind (Kap. 7.4.4.1 und 7.4.4.2). Stattdessen bestätigten verschiedene Indikatoren die These, dass die Studierenden den Wiki-Editor lediglich verwendeten, um ihre bereits erstellten Arbeitsergebnisse zu veröffentlichen. Die Nutzung des Wiki-Editors erfolgte nur von wenigen Studierenden. Zugleich wurde sichtbar, dass die Wikibookianerinnen und Wikibookianern auf vielen Seiten aktiv waren und Überarbeitungen der 
studentischen Beiträge vornahmen (Kap. 7.4.4). Erfahrungen zur Partizipation an einer Wiki-Community sowie zur Prozessualität von Wikiseiten waren Studierenden in unterschiedlicher Weise zugänglich. Belege für diese Aussagen lieferten (a) die Bearbeitungsstatistiken relevanter Wikiseiten sowie (b) exemplarische Auswertungen zur Aktivität der Bearbeiterinnen und Bearbeitern für ausgewählte Wikiseiten.

Die verschiedenen Ergebnisse der empirischen Analysen werden im folgenden Kapitel «Auswertung und Neuperspektivierung» (Kap. 8) aufgegriffen und vor dem Hintergrund des formulierten Entwurfs (Kap. 6) diskutiert und interpretiert. Mit der Fokussierung der zwei Zielstellungen des Entwurfs (Kap. 6.2) wird im Sinne von Sesink und Reinmann (2015, 81 f.) diskutiert, «ob das eingetreten ist, was der Entwurf vorgesehen hatte». Dies erfolgt in vergleichender Betrachtung der zuvor vorgestellten Ergebnisse. Ferner werden die Ergebnisse zukunftsorientiert interpretiert, um erste Hinweise zur Modifikation des Entwurfs zu entwickeln (Kap. 9).

\section{Literatur}

Anderson, Paul. 2007. What is Web 2.0: Ideas, technologies and implications for education. JISC. Technology \& Standards Watch. http://www.ictliteracy.info/rf.pdf/Web2.0_research.pdf.

Arnold, Patricia. 2011. «Die «Netzgeneration〉 - Empirische Untersuchungen zur Mediennutzung bei Jugendlichen». In Lehrbuch für Lernen und Lehren mit Technologien, herausgegeben von Sandra Schön und Martin Ebner, 1-7. L3T. http://l3t.tugraz.at/index.php/LehrbuchEbner10/ article/view/61.

Arslan, Derya, und Zeynep Karatas. 2015. «Mirror of Prospective Teachers' Mind: Metaphors». Procedia - Social and Behavioral Sciences 197 (Supplement C): 1464-71. https://doi. org/10.1016/j.sbspro.2015.07.096.

Berlanga, Adriana J., Francisco García Peñalvo, und Peter B. Sloep. 2010. «Towards eLearning 2.0 University». Interactive Learning Environments 18: 199-201.

Biermann, Ralf, Johannes Fromme, und Dan Verständig. 2014. «Partizipative Medienkulturen als Transformation von Beteiligungsmöglichkeiten - Einleitung». In Partizipative Medienkulturen: Positionen und Untersuchungen zu veränderten Formen öffentlicher Teilhabe, herausgegeben von Ralf Biermann, Johannes Fromme, und Dan Verständig, 7-17. Wiesbaden: Springer Fachmedien Wiesbaden. https://doi.org/10.1007/978-3-658-01793-4_1.

Blömeke, Sigrid. 2004. «Empirische Befunde zur Wirksamkeit der Lehrerbildung». In Handbuch Lehrerbildung, herausgegeben von Sigrid Blömeke, Peter Reinhold, Gerhard Tulodziecki, und Johannes Wildt, 59-91. Bad Heilbrunn: Julius Klinkhard Verlag.

Blömeke, Sigrid. 2005. «Medienpädagogische Kompetenz: Theoretische Grundlagen und erste empirische Befunde». In Kompetenzdiagnostik: Theorien und Methoden zur Erfassung und Bewertung von beruflichen Kompetenzen, herausgegeben von Andreas Frey, 1. Aufl., 76-97. Landau in der Pfalz: Empirische Pädagogik. 
Bohnsack, Ralf. 2010. «Fokussierungsmetapher». In Hauptbegriffe Qualitativer Sozialforschung, 3., durchges. Aufl., 8226:67. UTB. Opladen [et al.]: Bohnsack, Ralf and Marotzki, Winfried and Meuser, Michael.

Bonk, Curtis J., Mimi Miyoung Lee, Nari Kim, und Meng-Fen Grace Lin. 2009. «The tensions of transformation in three cross-institutional wikibook projects». The Internet and Higher Education 12 (3-4): 126-35. http://dx.doi.org/10.1016/j.iheduc.2009.04.002.

Bremer, Helmut. 2001. «Zur Theorie und Empirie der typenbildenden Mentalitätsanalyse». PhD Thesis, Universität Hannover. http://d-nb.info/962077151/34.

Budka, Philipp, Martin Ebner, Walther Nagler, und Claudia Schallert. 2011. «Hochschule - Strukturen, Rahmen und Modelle für die Lehre mit Technologien». In Lehrbuch für Lernen und Lehren mit Technologien, herausgegeben von Sandra Schön und Martin Ebner, 1-9. Graz: L3T. http://3t.eu/homepage/das-buch/ebook/kapitel/o/id/58/name/hochschule.

Dann, Hanns-Dietrich. 1989. «Subjektive Theorien als Basis erfolgreichen Handelns von Lehrkräften». Beiträge zur Lehrerinnen- und Lehrerbildung 7 (2): 247-254.

Duit, Reinders. 2010. «PIKO-BRIEF NR. 1: Schülervorstellungen und Lernen von Physik». In PikoBriefe. Der fachdidaktische Forschungsstand kurzgefasst, herausgegeben von Reinders Duit. Kiel: IPN Kiel. https://www.ipn.uni-kiel.de/de/das-ipn/abteilungen/didaktik-der-physik/ piko/pikobriefe032010.pdf.

Ebner, Martin, Mandy Schiefner, und Walther Nagler. 2008. «Has the Net Generation arrived at the University? - oder Studierende von heute, Digital Natives?» In Offener Bildungsraum Hochschule. Freiheiten und Notwendigkeiten - Tagungsband der GMW-Jahrestagung 2008, herausgegeben von Sabine Zauchner, Peter Baumgartner, Edith Blaschitz, und Andreas Weissenböck, 114-23. Medien in der Wissenschaft. Münster [et al.]: Waxmann.

Ehlers, Ulf-Daniel. 2010. «Qualität für digitale Lernwelten: Von der Kontrolle zur Partizipation und Reflexion». In Digitale Lernwelten: Konzepte, Beispiele und Perspektiven, herausgegeben von Kai-Uwe Hugger und Markus Walber, 59-73. Wiesbaden: VS Verlag für Sozialwissenschaften. https://doi.org/10.1007/978-3-531-92365-9_4.

Eickelmann, Birgit, Julia Gerick, und Wilfried Bos. 2014. «Die Studie ICILS 2013 im Überblick - Zentrale Ergebnisse und Entwicklungsperspektiven». In ICILS 2013 : computer- und informationsbezogene Kompetenzen von Schülerinnen und Schülern in der 8, herausgegeben von Wilfried Bos, Birgit Eickelmann, Julia Gerick, Frank Goldhammer, Heike Schaumburg, Knut Schwippert, Martin Senkbeil, und Renate Schulz-Zander, 9-31. Waxmann-E-Books : Empirische Erziehungswissenschaft. Münster [et al.]: Waxmann. http://www.content-select.com/ index.php?id=bib_view\&ean=9783830981312.

Eimeren, Birgit van, und Beate Frees. 2010. «Fast 50 Millionen Deutsche online - Multimedia für alle?» Media Perspektiven, Nr. 7-8: 334-49.

Grafe, Silke. 2008. Förderung von Problemlösefähigkeit beim Lernen mit Computersimulationen: Grundlagen und schulische Anwendungen. Klinkhardt Forschung. Bad Heilbrunn: Klinkhardt.

Grell, Petra. 2006. Forschende Lernwerkstatt. Bd. 472. Internationale Hochschulschriften. Münster [et al.]: Waxmann. 
Grell, Petra, und Franco Rau. 2011. «Partizipationslücken - Social Software in der Hochschullehre». MedienPädagogik: Zeitschrift für Theorie und Praxis der Medienbildung 21 (Partizipationschancen): 1-23. https://doi.org/10.21240/mpaed/21/2011.11.21.X.

Gropengießer, Harald. 2004. «Denkfiguren im Lehr-Lernprozess». In Lehren fürs Leben : didaktische Rekonstruktion in der Biologie, herausgegeben von Harald Gropengießer, 8-24. Köln: Aulis Verlag Deubner.

Grosch, Michael, und Gerd Gidion. 2011. Mediennutzungsgewohnheiten im Wandel: Ergebnisse einer Befragung zur studiumsbezogenen Mediennutzung. KIT Scientific Publishing, Karlsruhe. https://doi.org/10.5445/KSP/1000022524.

Gudjons, Herbert, und Rainer Winkel, Hrsg. 2006. Didaktische Theorien. 12. Aufl., Neuaufl. Bd. 1. PB-Buch. Hamburg: Bergmann + Helbig.

Guerrero, María C. M. de, und Olga S. Villamil. 2000. «Exploring ESL Teachers' Roles through Metaphor Analysis». TESOL Quarterly 34 (2): 341-51.

Guerrero, María C. M. de, und Olga S. Villamil. 2002. «Metaphorical conceptualizations of ESL teaching and learning». Language Teaching Research 6 (2): 95-120. https://doi. org/10.1191/1362168802/r1010a.

Guth, Sarah, und Corrado Petrucco. 2009. «Social Software and Language Acquisition». In Handbook of Research on E-Learning Methodologies for Language Acquisition, herausgegeben von R. Càssia Veiga Marriott und P. L. Torres, 424-42. New York: Information Science Reference.

Heinmann, Paul. 1962. «Didaktik als Theorie und Lehre». Die Deutsche Schule 54 (9): 407-427.

Herzig, Bardo. 1998. Förderung ethischer Urteils- und Orientierungsfähigkeit, Grundlagen und schulische Anwendungen. Förderung ethischer Urteils- und Orientierungsfähigkeit. Internationale Hochschulschriften;268. Münster [et al.]: Waxmann.

Herzig, Bardo, und Silke Grafe. 2007. Digitale Medien in der Schule: Standortbestimmung und Handlungsempfehlungen für die Zukunft. Bonn: Deutsche Telekom.

Hinze, Udo. 2008. «Computerbasiertes kooperatives Lernen (CSCL) als technische und pädagogische Herausforderung». In Internet - Bildung - Gemeinschaft, herausgegeben von Friederike von Gross, Winfried Marotzki, und Uwe Sander, 241-261. Wiesbaden: VS Verlag für Sozialwissenschaften. https://doi.org/10.1007/978-3-531-90793-2_12.

Horstkemper, Marianne. 2004. «Erziehungswissenschaftliche Ausbildung». In Handbuch Lehrerbildung, herausgegeben von Sigirid Blömeke, Peter Reinhold, Gerhard Tulodziecki, und Johannes Wildt, 461-76. Bad Heilbrunn: Julius Klinkhardt.

Iske, Stefan, und Winfried Marotzki. 2010. «Wikis: Reflexivität, Prozessualität und Partizipation». In Medienbildung in neuen Kulturräumen. Die deutschprachige und britische Diskussion, herausgegeben von Ben Bachmair, 1. Aufl., 141-151. Wiesbaden: VS Verlag für Sozialwissenschaften. https://doi.org/10.1007/978-3-531-92133-4_10.

Jank, Werner, und Hilbert Meyer. 2008. Didaktische Modelle. Berlin: Cornelsen.

Jenkins, Henry. 2007. The Power of "Collegial Pedagogy»: An Interview with Youth Radio (Part One). http://www.henryjenkins.org/2007/08/youth_radio.html.

Jones, Norah, Haydn Blackey, Karen Fitzgibbon, und Esyin Chew. 2010. «Get out of MySpace!» Computers \& Education 54 (3): 776-82. https://doi.org/10.1016/j.compedu.2009.07.008. 
Kammerl, Rudolf, und Sandra Ostermann. 2010. Medienbildung - (k)ein Unterrichtsfach? Eine Expertise zum Stellenwert der Medienkompetenzförderung in Schulen. Hamburg: Medienanstalt Hamburg/Schleswig-Holstein (MA HSH).

Kammerl, Rudolf, und Simon Pannarale. 2007. «Students in Higher Education and Teacher Training Programs in Germany: Their Internet Use, Media Literacy, and Attitude towards eLearning». In Proceedings of Society for Information Technology \& Teacher Education International Conference 2007, herausgegeben von Roger Carlsen, Karen McFerrin, Jerry Price, Roberta Weber, und Dee Anna Willis, 3067-3072. San Antonio, Texas, USA: Association for the Advancement of Computing in Education (AACE). https://www.learntechlib.org/p/25073.

Kerres, Michael. 2013. Mediendidaktik: Konzeption und Entwicklung mediengestützter Lernangebote. 4., überarb. und aktualisierte Aufl. München.

Klafki, Wolfgang. 1991. Neue Studien zur Bildungstheorie und Didaktik: zeitgemäße Allgemeinbildung und kritisch-konstruktive Didaktik. 2., erw. Aufl. Reihe Pädagogik. Weinheim [et al.]: Beltz.

Klafki, Wolfgang. 1997. «Die bildungstheoretische Didaktik im Rahmen kritisch-konstruktiver Erziehungswissenschaft». In Didaktische Theorien, herausgegeben von Herbert Gudjons und Rainer Winkel, 9. Auflage, 13-34. Hamburg: Bergmann und Helbig.

Kleimann, Bernd, Murat Özkilic, und Marc Göcks. 2008. Studieren im Web 2.0. Studienbezogene Web- und E-Learning-Dienste. Kurzinformation Nr. 21. HISBUS. http://www.hisbus.de/ pdf/2008_07_hisbus_web2.0.pdf.

Klingberg, Lothar. 1990. Lehrende und Lernende im Unterricht. Berlin: Volk und Wissen Verlag.

KMK, Kultusministerkonferenz. 2014. Standards der Lehrerbildung: Bildungswissenschaften. Kultusministerkonferenz. http://www.kmk.org/fileadmin/Dateien/veroeffentlichungen_ beschluesse/2004/2004_12_16-Standards-Lehrerbildung-Bildungswissenschaften.pdf.

Kromrey, Helmut. 2009. Empirische Sozialforschung: Modelle und Methoden der standardisierten Datenerhebung und Datenauswertung. 12., überarb. und erg. Aufl. Bd. 1040 : Soziologie. UTB. Stuttgart: Lucius \& Lucius.

Kron, Friedrich W., Jutta Strandop, und Jürgens Eiko. 2014. Grundwissen Didaktik. 6. Auflage. München, Basel: Reinhardt.

Kruse, Otto. 2007. Keine Angst vor dem leeren Blatt : ohne Schreibblockaden durchs Studium. 12., völlig neu bearb. Aufl. Frankfurt [et al.]: Campus Verlag.

Kuckartz, Udo, Thorsten Dresing, Stefan Rädiker, und Claus Stefer. 2008. Qualitative Evaluation : der Einstieg in die Praxis. 2., aktualisierte Aufl. Wiesbaden: Springer VS.

Künkler, Tobias. 2011. Lernen in Beziehung. Zum Verhältnis von Subjektivität und Relationalität in Lernprozessen. Bielefeld: transcript Verlag.

Marsch, Sabine. 2009. «Metaphern des Lehrens und Lernens. Vom Denken, Reden und Handeln bei Biologielehrern». PhD Thesis, Berlin: Freie Universität Berlin.

Marsch, Sabine, und Dirk Krüger. 2007. «Vorstellungen von Biologielehrern». In Lehr- und Lernforschung in der Biologiedidaktik. Ausbildung und Professionalisierung von Lehrkräften, herausgegeben von Ute Harms und Angela Sandmann, 253-272. Innsbruck, Wien, Bozen: Studienverlag. 
Martinez, Maria A., Narcis Sauleda, und Günter L. Huber. 2001. «Metaphors as blueprints of thinking about teaching and learning». Teaching and Teacher Education 17 (8): 965-77. https:// doi.org/10.1016/S0742-051X(01)00043-9.

Mayrberger, Kerstin. 2012. «Medienpädagogische Kompetenz im Wandel - Vorschlag zur Gestaltung des Übergangs in der Lehrerbildung am Beispiel mediendidaktischer Kompetenz». In Jahrbuch Medienpädagogik 9, herausgegeben von Renate Schulz-Zander, Birgit Eickelmann, Heinz Moser, Horst Niesyto, und Petra Grell, 389-412. Wiesbaden: VS Verlag für Sozialwissenschaften. https://doi.org/10.1007/978-3-531-94219-3_17.

Mayrberger, Kerstin. 2013a. «Eine partizipative Mediendidaktik (nicht nur) für den Hochschulkontext?»In E-Learning zwischen Vision und Alltag. Zum Stand der Dinge, herausgegeben von C. Bremer und D. Krömker, 96-106. Münster [et al.]: Waxmann. https://www.waxmann. com/?elD=texte\&pdf=2953Volltext.pdf\&typ=zusatztext.

Mayrberger, Kerstin. 2013b. «Partizipatives Lernen mit dem Social Web in der Schule». In Organisation und Partizipation: Beiträge der Kommission Organisationspädagogik, herausgegeben von Susanne Maria Weber, Michael Göhlich, Andreas Schröer, Claudia Fahrenwald, und Hildegard Macha, 167-175. Wiesbaden: Springer Fachmedien Wiesbaden. https://doi. org/10.1007/978-3-658-00450-7_14.

Mayrberger, Kerstin, Stephan Waba, und Michael Schwratz. 2013. «Social Media in der Lehrerbildung. Editorial». Herausgegeben von Kerstin Mayrberger, Stephan Waba, und Michael Schwratz. Journal für LehrerInnenbildung 13 (4): 4-5.

Mayring, Philipp. 1990. Qualitative Inhaltsanalyse : Grundlagen und Techniken. 2., überarb. Aufl. Weinheim [et al.].

Mayring, Philipp. 2000. «Qualitative Content Analysis». Forum Qualitative Sozialforschung / Forum: Qualitative Social Research 1 (2). https://doi.org/10.17169/fqs-1.2.1089.

Mayring, Philipp. 2002. Einführung in die qualitative Sozialforschung : eine Anleitung zu qualitativem Denken. 5., überarb. und neu Ausgestattete Aufl. Beltz-Studium. Weinheim [et al.]: Beltz.

Moser, Heinz. 2010. Schule 2.0: Medienkompetenz für den Unterricht. 1. Aufl. Bd. 20. Schulmanagement konkret. Kronach: Link.

Niesyto, Horst. 2012. «Medienpädagogik in der Lehrerbildung in Baden-Württemberg. Konzeptionelle Überlegungen und praktische Schritte zu einer medienpädagogischen Grundbildung». In Jahrbuch Medienpädagogik 9, herausgegeben von Renate Schulz-Zander, Birgit Eickelmann, Heinz Moser, Horst Niesyto, und Petra Grell, 333-357. Wiesbaden: VS Verlag für Sozialwissenschaften. https://doi.org/10.1007/978-3-531-94219-3_15.

Pajares, M. Frank. 1992. «Teachers' Beliefs and Educational Research: Cleaning Up a Messy Construct». Review of Educational Research 62 (3): 307-32. https://doi. org/10.3102/00346543062003307.

Peterhans, Matthias, und Stefanie Sagl. 2011. (N)Onliner Atlas 2011. Initiative D21 e.V. und TNS Infratest. https://initiatived21.de/publikationen/nonliner-atlas-2011/.

Peterssen, Wilhelm. 2001. Lehrbuch Allgemeine Didaktik. Oldenbourg Wissenschaftsverlag $\mathrm{GmbH}$. 
Petko, Dominik. 2011. «Praxisorientierte medienpädagogische Forschung: Ansätze für einen empirischen Perspektivenwechsel und eine stärkere Konvergenz von Medienpädagogik und Mediendidaktik». MedienPädagogik: Zeitschrift für Theorie und Praxis der Medienbildung 20 (0): 245-258. https://doi.org/10.21240/mpaed/20/2011.09.22.X.

Peyer, Ann, und Rudolf Künzli. 1999. «Metaphern in der Didaktik». Zeitschrift für Pädagogik 45 (2): 177-194.

Poom-Valickis, Katrin, Tuuli Oder, und Madis Lepik. 2012. «Teachers' Beliefs Regarding their Professional Role: A Gardener, Lighthouse or Circus Director?» Procedia - Social and Behavioral Sciences 69 (Supplement C): 233-41. https://doi.org/10.1016/j.sbspro.2012.11.404.

Prensky, Marc. 2001. «Digital Natives, Digital Immigrants». On the Horizon 9 (5): 1-6.

Rau, Franco. 2013. «Social Software in der Hochschullehre : kritische Analyse didaktischer Szenarien». Universität Potsdam. https://publishup.uni-potsdam.de/opus4-ubp/frontdoor/index/index/year/2013/docld/6202.

Reich, Kersten. 2012. Konstruktivistische Didaktik: das Lehr- und Studienbuch mit Online-Methodenpool. 5., erw. Aufl. Weinheim [et al.]: Beltz Pädagogik.

Reinmann, Gabi. 2010. "Selbstorganisation auf dem Prüfstand: Das Web 2.0 und seine Grenzen(losigkeit)». In Digitale Lernwelten: Konzepte, Beispiele und Perspektiven, herausgegeben von Kai-Uwe Hugger und Markus Walber, 75-89. Wiesbaden: VS Verlag für Sozialwissenschaften. https://doi.org/10.1007/978-3-531-92365-9_5.

Saban, Ahmet, Beyhan Nazli Kocbeker, und Aslihan Saban. 2007. «Prospective teachers' conceptions of teaching and learning revealed through metaphor analysis». Learning and Instruction 17 (2): 123-39. https://doi.org/10.1016/j.learninstruc.2007.01.003.

Schiefner-Rohs, Mandy. 2012. Kritische Informations- und Medienkompetenz Theoretisch-konzeptionelle Herleitung und empirische Betrachtungen am Beispiel der Lehrerausbildung. Internationale Hochschulschriften. Münster [et al.]: Waxmann.

Schiefner-Rohs, Mandy. 2013. "Das Social Web als Erfahrungsraum für die Lehrerbildung - Medienbildung zwischen Werkzeug und Raum». journal für lehrerInnenbildung 13 (4): 6-12.

Schmitt, Rudolf. 2017. Systematische Metaphernanalyse als Methode der qualitativen Sozialforschung. Wiesbaden: Springer Fachmedien Wiesbaden. https://doi.org/10.1007/978-3-65813464-8.

Schorb, Bernd. 2008. «Handlungsorientierte Medienpädagogik». In Handbuch Medienpädagogik, herausgegeben von Uwe Sander, Friederike von Gross, und Kai-Uwe Hugger, 1. Aufl., 75-86. Wiesbaden: VS Verlag für Sozialwissenschaften.

Schulmeister, Rolf. 2009a. «Studierende, Internet, E-Learning und Web 2.0». In E-Learning 2009. Lernen im digitalen Zeitalter, herausgegeben von Nicolas Apostolopoulos, Harriet Hoffmann, Veronika Mansmann, und Andreas Schwill, 129-140. Medien in der Wissenschaft. Münster [et al.]: Waxmann.

Schulmeister, Rolf. 2009b. Gibt es eine »Net Generation«? Erweiterte Version 3.0. Universität Hamburg, Zentrum für Hochschul- und Weiterbildung. http://epub.sub.uni-hamburg.de/ epub/volltexte/2013/19651/pdf/schulmeister_net_generation_v3.pdf. 
Schulz, Wolfgang. 2006. «Die lehrtheoretische Didaktik». In Didaktische Theorien, herausgegeben von Herbert Gudjons und Rainer Winkel, 12., 35-56. Hamburg: Bergmann und Helbig.

Sesink, Werner, und Gabi Reinmann. 2015. «Umrisse eines Strukturmodells für entwicklungsorientierte bildungswissenschaftliche Forschung». In Entwicklungsorientierte Bildungsforschung. Plädoyer für einen ‘dritten Weg〉 in pädagogischer Forschung. Eine Textsammlung, herausgegeben von Werner Sesink, 69-83. http://www.sesink.de/wordpress/wp-content/ uploads/2015/11/Entwicklungsorientierte-Bildungsforschung_Sesink_2015.pdf.

Telekom Stiftung. 2015. Schule digital Der Länderindikator 2015. Deutsche Telekom Stiftung. https://www.telekom-stiftung.de/sites/default/files/schuledigital_2015_web.pdf.

Tapscott, Don. 1998. Net Kids: Die digitale Generation erobert Wirtschaft und Gesellschaft. Wiesbaden: Gabler.

Terhart, Ewald. 1999. "Sprache der Erziehungswissenschaft. Eine Einführung in den Thementeil». Zeitschrift für Pädagogik 45 (2): 155-159.

Terhart, Ewald. 2009. Didaktik : eine Einführung. Stuttgart: Reclam.

Thomas, Lynn, und Catherine Beauchamp. 2011. «Understanding new teachers' professional identities through metaphor». Teaching and Teacher Education 27 (4): 762-69. https://doi. org/10.1016/j.tate.2010.12.007.

TU Darmstadt, Institut für Allgemeine Pädagogik und Berufspädagogik. 2009a. Studienordnung Lehramt an Gymnasien Grundwissenschaften. https://www.zfl.tu-darmstadt.de/media/zfl/ studium_medien/studium_lag/studium_lag_studienordnungen/grundwissenschaften/ Grundwissenschaften_LaG_Ausfuehrungsbestimmung_10-2005_SB_3-06.pdf.

TU Darmstadt, Institut für Allgemeine Pädagogik und Berufspädagogik. 2009b. Modulbeschreibungen Lehramt an Gymnasien Grundwissenschaften. https://www.zfl.tu-darmstadt.de/me$\mathrm{dia} / \mathrm{zfl} /$ studium_medien/studium_lag/studium_lag_studienordnungen/grundwissenschaften/Grundwissenschaften_LaG_Modulhandbuch_10-2005_SB_3-06.pdf.

Tulodziecki, Gerhard, Silke Grafe, und Bardo Herzig. 2013. Gestaltungsorientierte Bildungsforschung und Didaktik: Theorie - Empirie - Praxis. Bad Heilbrunn: Klinkhardt.

Tulodziecki, Gerhard. 2012. «Medienpädagogische Kompetenz und Standards in der Lehrerbildung». In Jahrbuch Medienpädagogik 9, herausgegeben von Renate Schulz-Zander, Birgit Eickelmann, Heinz Moser, Horst Niesyto, und Petra Grell, 271-297. Wiesbaden: VS Verlag für Sozialwissenschaften. https://doi.org/10.1007/978-3-531-94219-3_13.

Tulodziecki, Gerhard, Silke Grafe, und Bardo Herzig. 2014. «Praxis- und theorieorientierte Entwicklung und Evaluation von Konzepten für medienpädagogisches Handeln als gestaltungsorientierte Bildungsforschung.» In Jahrbuch Medienpädagogik. Methodologie und Methoden medienpädagogischer Forschung, herausgegeben von Anja Hartung, Bernd Schorb, Horst Niesyto, Heinz Moser, und Petra Grell, 10:213-229. Jahrbuch Medienpädagogik. Wiesbaden: Springer VS.

Tulodziecki, Gerhard, Bardo Herzig, und Silke Grafe. 2010. Medienbildung in Schule und Unterricht. Grundlagen und Beispiele. Bad Heilbrunn [et al.]: UTB / Klinkhardt. 
Wernke, Stephan, Jochen Werner, und Klaus Zierer. 2015. «Heimann, Schulz oder Klafki? Eine quantitative Studie zur Einschätzung der Praktikabilität allgemeindidaktischer Planungsmodelle.» Zeitschrift für Pädagogik.

Wikibooks. 2016. Hauptseite - Wikibooks, Die freie Bibliothek. https://de.wikibooks.org/w/index.php?title=Hauptseite \&oldid=800234.

Wildt, Johannes. 2002. «Ein hochschuldidaktischer Blick auf Lehren und Lernen». In Neues Handbuch Hochschullehre, herausgegeben von Brigitte Berendt, Hans-Peter Voss, und Johannes Wildt, 1-10. Bonn: Raabe-Verlag.

Wildt, Johannes. 2003. «Reflexives Lernen in der Lehrerbildung - ein Mehrebenenmodell in hochschuldidaktischer Perspektive». In Forschendes Lernen: Theorie und Praxis einer professionellen LehrerInnenausbildung, herausgegeben von Alexandra Obolenski und Hilbert Meyer, 71-84. Bad Heilbrunn: Klinkhardt.

Winkel, Rainer. 1997. «Die kritisch-kommunikative Didaktik». In Didaktische Theorien, herausgegeben von Herbert Gudjons und Rainer Winkel, 9. Auflage, 93-112. Hamburg: Bergmann und Helbig.

de Witt, Claudia, und Thomas Czerwionka. 2007. Mediendidaktik. Studientexte für Erwachsenenbildung. Bielefeld: wbv, Bertelsmann.

Xiao, Yun, und Robert Lucking. 2008. "The impact of two types of peer assessment on students' performance and satisfaction within a Wiki environment». The Internet and Higher Education 11 (3-4): 186-193. https://doi.org/10.1016/j.iheduc.2008.06.005.

Zawacki-Richter, Olaf, Günter Hohlfeld, und Wolfgang Müskens. 2014. «Mediennutzung im Studium». Schriftenreihe zum Bildungs- und Wissenschaftsmanagement 1 (1). http://openjournal. uni-oldenburg.de/index.php/bildungsmanagement/article/view/10. 